\title{
Hypnotika auf Privatrezept auch für Kassenpatienten
}

\section{- Motive einer Praxis}

\author{
INAUGURAL-DISSERTATION \\ zur Erlangung des Doktorgrades \\ der Medizinischen Fakultät der \\ Georg-August-Universität zu Göttingen
}

vorgelegt von

Katharina Schmalstieg

aus

Göttingen

Göttingen 2018 
Dekan:

\section{Betreuungsausschuss}

Betreuer/in

Ko-Betreuer/in:

\section{Prüfungskommission}

Referent/in

Ko-Referent/in:

Drittreferent/in:

Datum der mündlichen Prüfung:
Prof. Dr. rer. nat. H. K. Kroemer

Prof. Dr. med. E. Hummers-Pradier

Prof. Dr. med. J. Brockmöller 
Hiermit erkläre ich, die Dissertation mit dem Titel " Hypnotika auf Privatrezept auch für Kassenpatienten - Motive einer Praxis" eigenständig angefertigt und keine anderen als die von mir angegebenen Quellen und Hilfsmittel verwendet zu haben.

Göttingen, den

(Unterschrift) 


\section{Inhaltsverzeichnis}

Abbildungsverzeichnis .........................................................................................

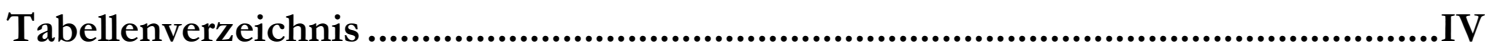

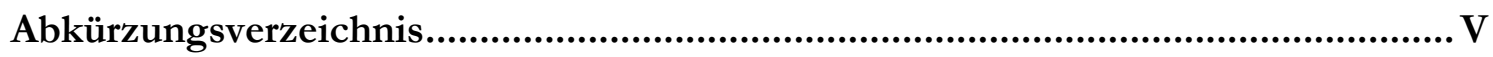

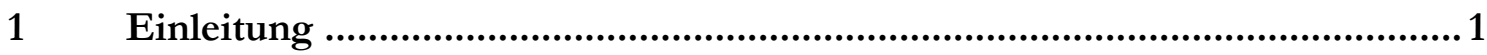

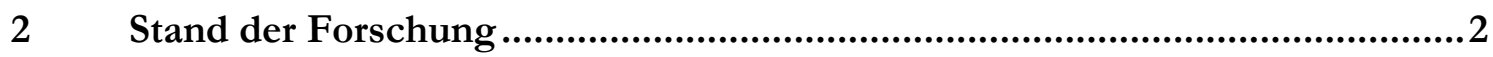

2.1 Verfügbare Benzodiazepine und Benzodiazepin-Rezeptoragonisten..........................................2

2.2 Leitlinien- und indikationsgerechter Gebrauch ......................................................................

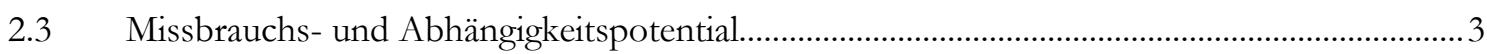

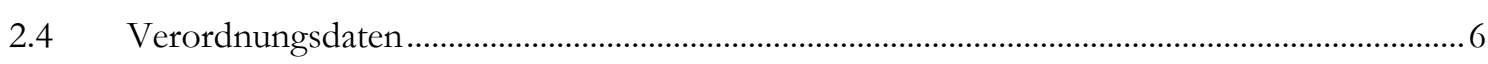

2.5 Einstellung von Ärzten gegenüber Hypnotika ....................................................................

2.5.1 Verschreibungsverhalten in Abhängigkeit vom Geschlecht des Arztes....................................10

2.5.2 Verschreibungsverhalten in Abhängigkeit vom Alter und der Ausbildung des Arztes .........10

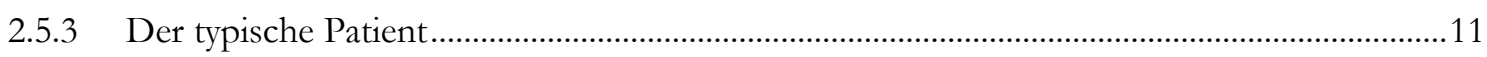

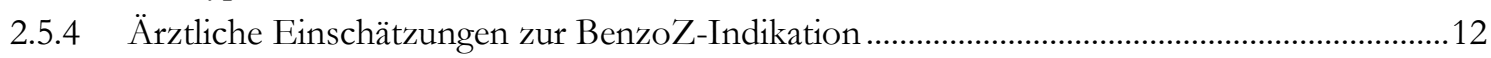

2.5.5 Ärztliche Wahrnehmung der Alternativen zu BenzoZ .........................................................14

2.5.6 Ärztliche Wahrnehmung der Risiken und Nebenwirkungen von BenzoZ...............................15

2.5.7 Übertragung der Verantwortung.......................................................................................... 17

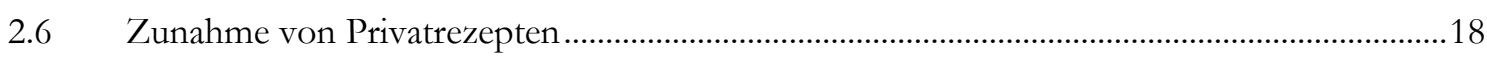

2.7 Motive für das Ausstellen eines Privatrezeptes ........................................................................19

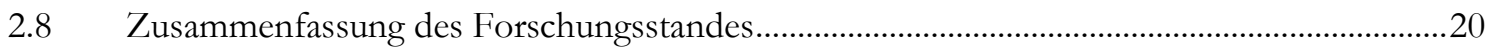

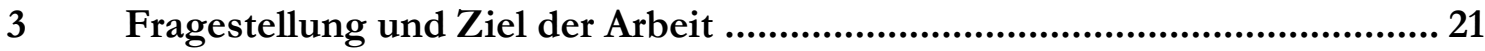

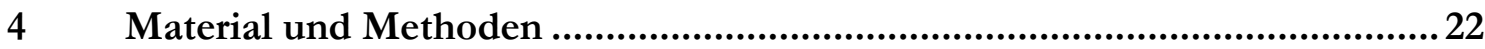

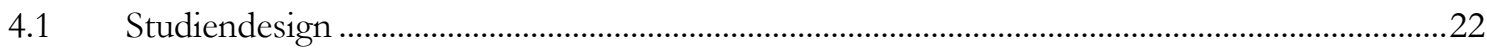

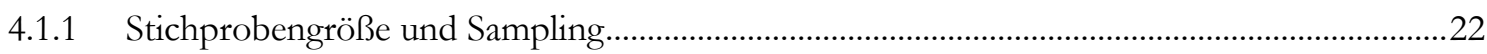

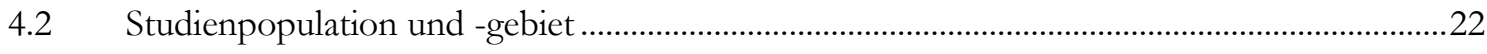

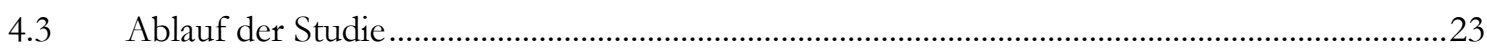

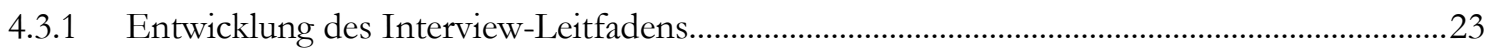

4.3.2 Rekrutierung der teilnehmenden Praxen und Ärzte ...............................................................23

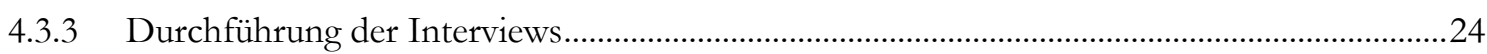

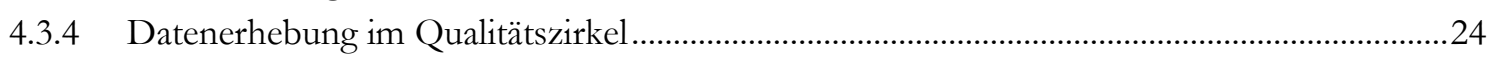

4.3.5 Datenrecherche zu KV-, Krankenkassen-, Apotheken- und

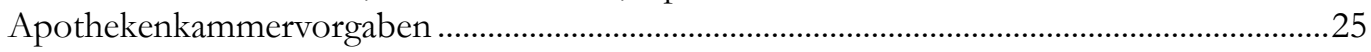

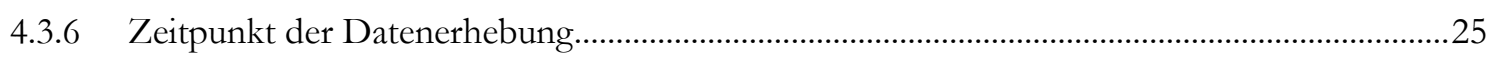

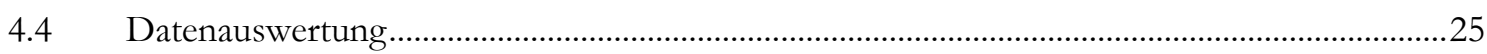

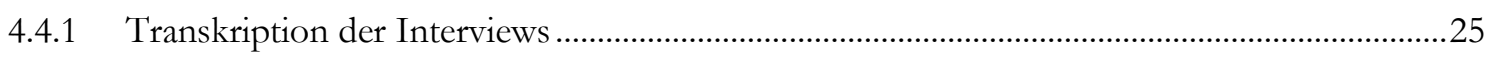




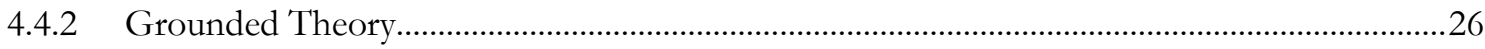

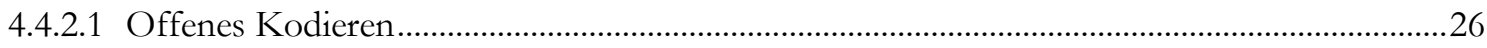

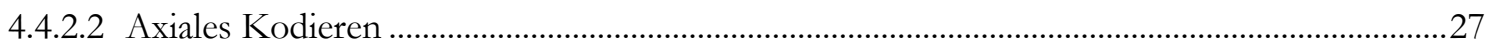

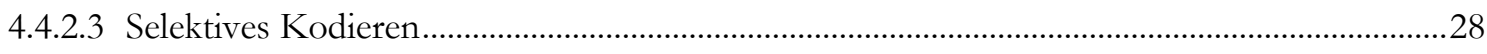

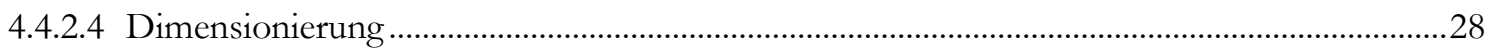

4.4.2.5 Überprüfung des Kodesystems .............................................................................................28

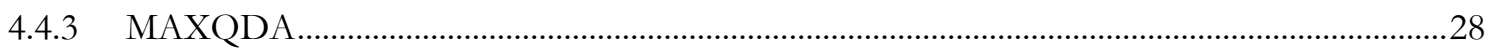

4.5 Datenschutz und Genehmigung durch die Ethikkommission.............................................29

4.6 Vorstellung und Veröffentlichung der Daten .....................................................................29

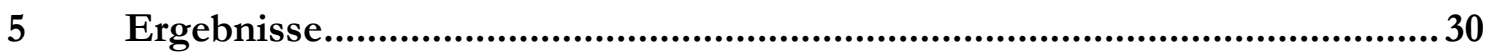

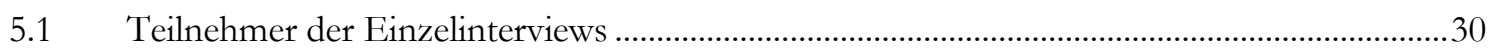

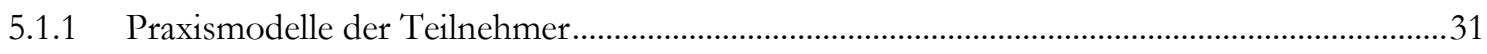

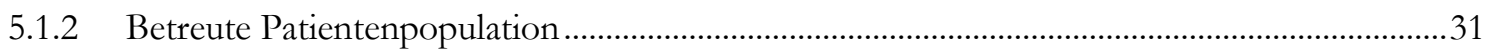

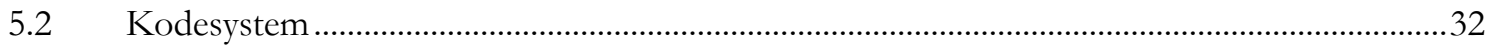

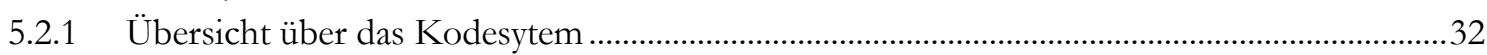

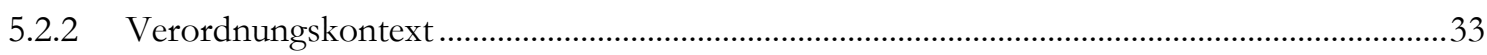

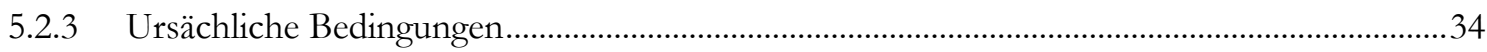

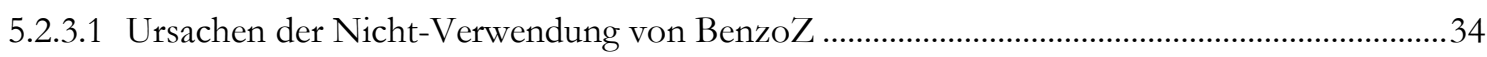

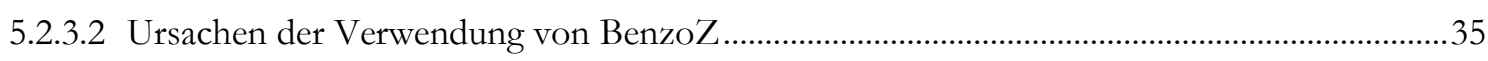

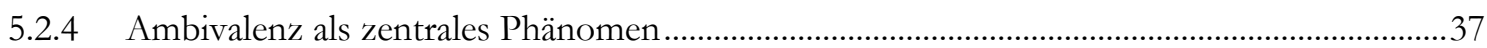

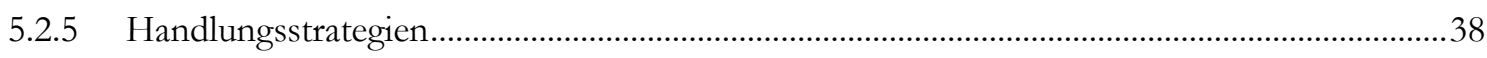

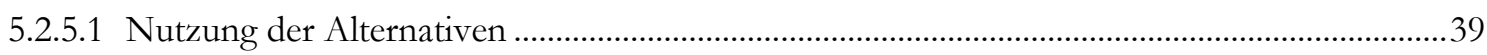

5.2.5.2 Nutzung der Benzodiazepine und Z-Substanzen ..............................................................40

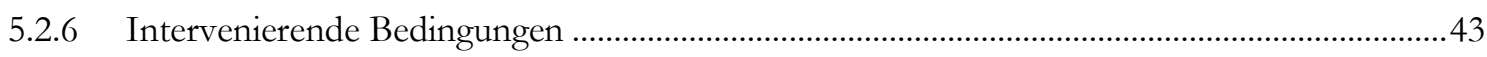

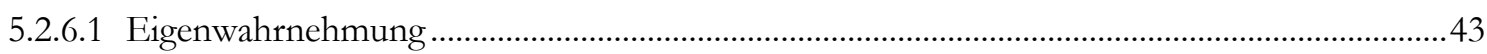

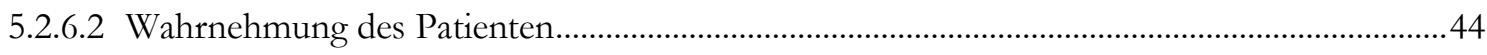

5.2.6.3 Wahrnehmung der Kollegen und Mitbetreuer .......................................................................4

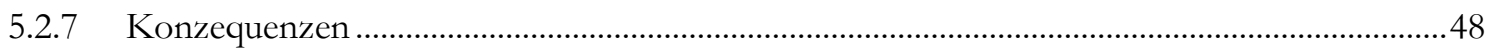

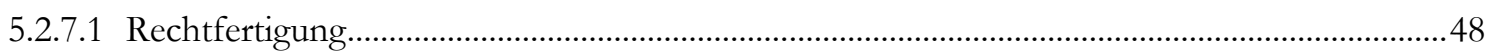

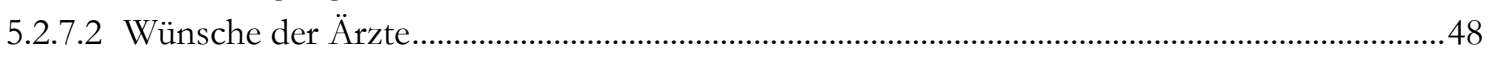

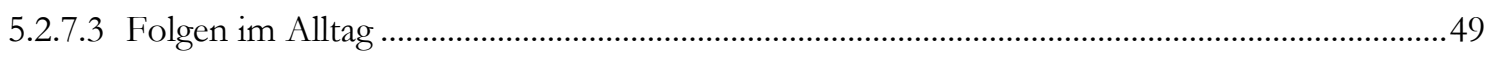

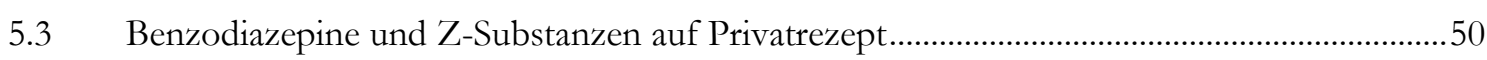

5.3.1 Privatrezept auf Grund von Unsicherheit bezüglich bestehender Richtlinien........................51

5.3.2 Privatrezept nutzen, um eine Hemmschwelle $\mathrm{zu}$ kreieren.......................................................52

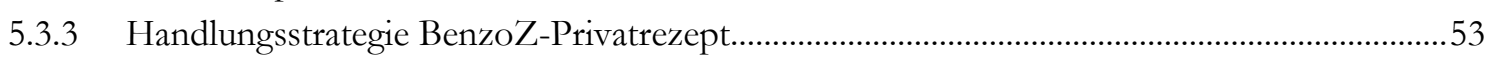

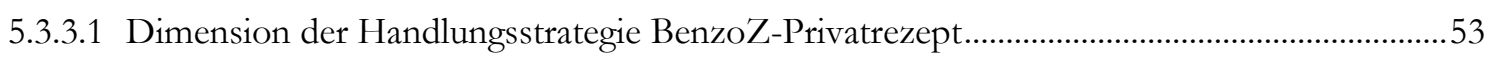

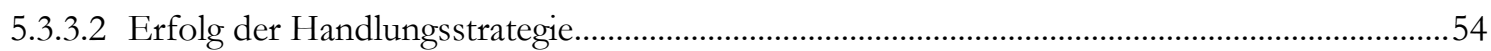

5.3.4 Privatrezept in Abhängigkeit vom Patienten(typ) ....................................................................5

5.3.5 Privatrezept in Abhängigkeit von der Diagnose und Problemdauer ........................................55

5.3.6 Privatrezept in Abhängigkeit von der Substanzklasse ...............................................................56

5.3.7 Privatrezept und Routine - Einflussfaktor oder Folge? ..............................................................5

5.3.8 Privatrezept zur Entlastung der Versichertengemeinschaft ......................................................57

5.3.9 Privatrezept zur Budgetentlastung und Regressvermeidung....................................................58

5.4 Das Privatrezept als Thema im Qualiätszirkel.......................................................................59 


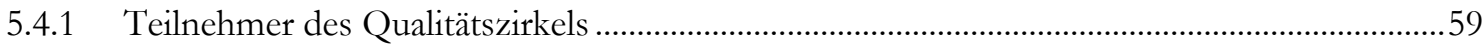

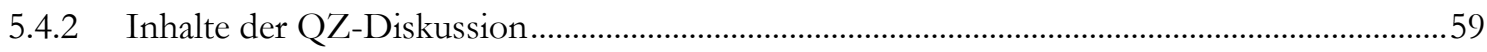

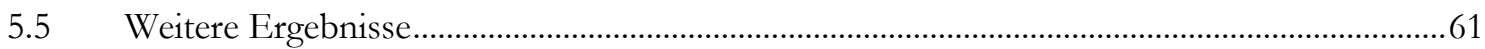

5.5.1 Das Privatrezept aus der Laien-/Bürgerperspektive .................................................................61

5.5.2 Auskunft von Kassenärztlichen Vereinigungen ...................................................................63

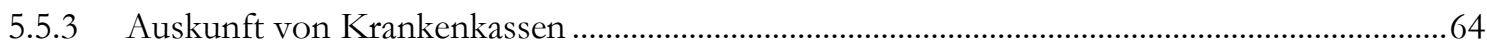

5.5.4 Auskunft von Apotheke und Apothekenkammer ................................................................67

6 Diskussion...........................................................................................69

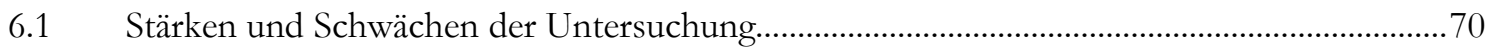

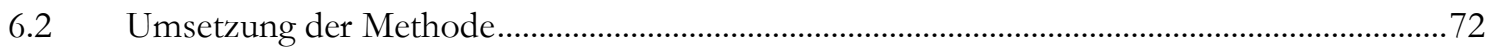

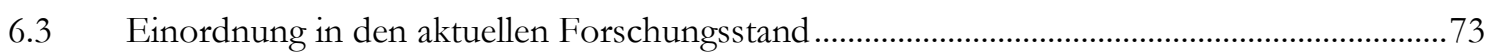

6.3.1 Einordnung in den Forschungsstand zur Einstellung von Ärzten..........................................73

6.3.1.1 BenzoZ-Verordnung im ländlichen und städtischen Gebiet ...............................................77

6.3.2 Einordnung in den Forschungsstand zur Ambivalenz ........................................................77

6.3.3 Einordnung in den Forschungsstand zum Privatrezept ........................................................79

6.4 Das Privatrezept zur Konsumeinschränkung? .................................................................... 82

6.4.1 Sorgloser Umgang mit selbstbezahlten Medikamenten? ......................................................84

6.5 Keine Orientierungshilfe zur Versorgung geerbter Patienten.............................................85

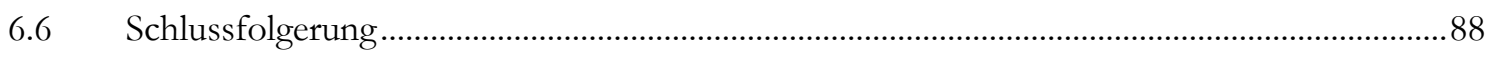

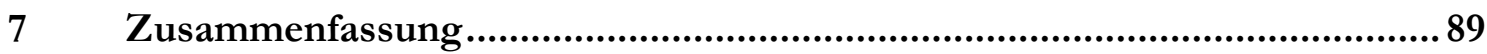

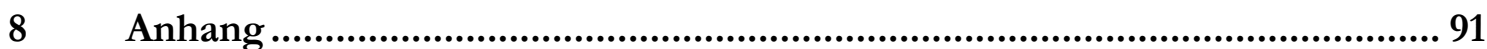

8.1 Artikelliste zu 2.5: Eistellung von Ärzten gegenüber Hypnotika...........................................91

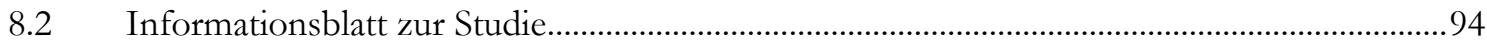

$8.3 \quad$ Einverständniserklärung Einzelinterviews .....................................................................96

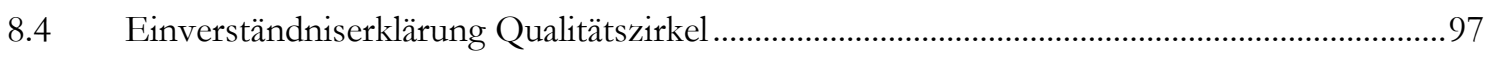

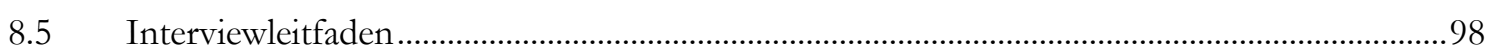

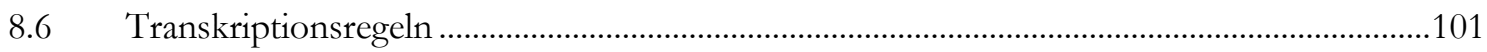

8.7 COREQ-Checkliste zur Darstellung qualitativer Forschungsergebnisse..............................102

$9 \quad$ Literaturverzeichnis .........................................................................105 


\section{Abbildungsverzeichnis}

Abbildung 1: Übersichtsdarstellung der relevanten Artikel in 2.5 _.....................................................

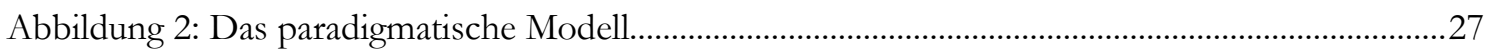

Abbildung 3: Übersicht der Ergebnisse dargestellt an Hand des paradigmatische Modells ................32

Abbildung 4: Übersicht der Subkategorien des Verordnungskontextes..................................................33

Abbildung 5: Subkategorien der indikationsbezogenen Ursachen der Nicht-Verwendung von

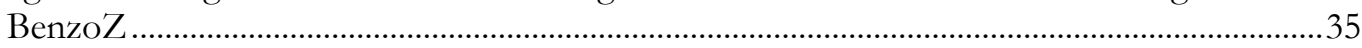

Abbildung 6: Subkategorien der indikationsbezogenen Ursachen der Verwendung von

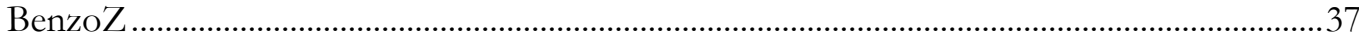

Abbildung 7: Die Dimension der Handlungsstrategie ,Nutzung der BenzoZ‘ .......................................40

Abbildung 8a: Übersicht der im Vorfeld genutzten Strategien............................................................... 40

Abbildung 8b: Darstellung der im Verlauf verwendeten Strategien, wenn bereits ein

Benzodiazepin oder eine Z-Substanz eingenommen wurden .....................................................42

Abbildung 8c: Übersicht der Strategien, die zur Minimierung der verordneten Menge an Benzodiazepinen und Z-Substanzen dienen ..........................................................................4

Abbildung 9: Von den Teilnehmern wahrgenommene Patiententypen .................................................45

Abbildung 10: Paradigmatisches Modell der BenzoZ-Privatverordnung................................................51

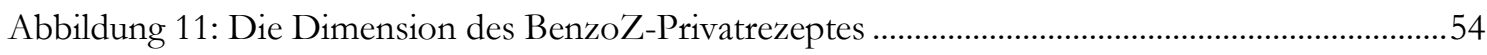

Abbildung 12: Auszug aus der Anlage III der Arzneimittelrichtlinie....................................................87

\section{Tabellenverzeichnis}

Tabelle 1: Darstellung der Medical Subject Headings (MeSH)-Suche in Pubmed...................................7

Tabelle 2: Demographische Daten der Studienteilnehmer ....................................................................30 


\section{Abkürzungsverzeichnis}

AIS: Arztpraxisinformationssystem

AMRL: Arzneimittelrichtlinie

ATC: anatomisch-therapeutisch-chemisch

AWMF: Arbeitsgemeinschaft der Wissenschaftlichen Medizinischen Fachgesellschaften

BÄK: Bundesärztekammer

BenzoZ: Benzodiazepine und Z-Substanzen

BMG: Bundesgesundheitsministerium

BZD: Benzodiazepin/-e

DDD: defined daily dose (definierte Tagesdosis)

DGPPN: Deutsche Gesellschaft für Psychiatrie und Psychotherapie, Psychosomatik und Nervenheilkunde

DMP: Disease Management Program

DSM-IV: Diagnostic and Statistical Manual of Mental Disorders, 4.Überarbeitung

GKV: gesetzliche Krankenversicherung

I: Interviewer

ICD-10: International Statistical Classification of Diseases and Related Health Problems, 10. Überarbeitung

K(B)V: Kassenärztliche (Bundes-)Vereinigung

KVB: Kassenärztliche Vereinigung Bayern

KVN: Kassenärztliche Vereinigung Niedersachsen

MDK: Medizinscher Dienst der Krankenkassen

MeSH: Medical Subject Headings - der durch die National Library of Medicine kontrollierte Thesaurus, der zu Indexierung von Pubmed-Artikeln dient

P: interviewte Person

QZ: Qualitätszirkel

Tn: Teilnehmerin

WIdO: Wissenschaftliches Institut der AOK 


\section{$1 \quad$ Einleitung}

„Jeder Versuch, das Verschreibungsverhalten von Ärzten ${ }^{1}$ zu beeinflussen, sollte auf einem genauen Verständnis darüber basieren, wie Verschreibungsentscheidungen eigentlich getroffen werden.“(Bradley 1992b)

Benzodiazepinhypnotika und Benzodiazepin-Rezeptoragonisten wie Zolpidem und Zopiclon (Z-Substanzen) werden auf Grund unterschiedlichster Indikationen verordnet. Laut des Bundesgesundheitssurveys von 2003 sind aber Schlafstörungen sowie Erregungs- und Spannungszustände, innere Unruhe und Nervosität die häufigsten Einnahmegründe (Knopf und Melchert 2003), und dies obwohl ein Nutzen der Hypnotika bei langfristiger Anwendung zur Therapie von Schlafstörungen nicht wissenschaftlich dokumentiert ist. Hingegen gibt es zahlreiche Studien, die die erheblichen unerwünschten Nebenwirkungen, u. a. das Missbrauchs- und Abhängigkeitspotential oder das vermehrte Sturzrisiko belegen, auch unter Einnahme der vergleichsweise neueren Z-Substanzen, die teilweise heute noch von vielen Ärzten als nebenwirkungsärmer eingeschätzt werden (Hoffmann 2013a). Die deutsche Gesellschaft für Schlafforschung und Schlafmedizin empfiehlt daher in ihrer Leitlinie von 2009 sowie im Update von 2016 kognitiv-verhaltenstherapeutische Maßnahmen zur Behandlung von primären Insomnien vor jeglicher Pharmakotherapie (S3-Leitlinie Nicht erholsamer Schlaf/Schlafstörungen 2009, 2017). Sowohl nach der Leitlinie der Deutschen Gesellschaft für Neurologie als auch nach der aktuellen Arzneimittel-Richtlinie (AMRL) von 2008 (letzte Änderung 26.02.2016) sollten Hypnotika nur zur Kurzzeittherapie von bis zu vier Wochen genutzt werde; länger andauernde Behandlungen sind aber in „medizinisch begründeten Einzelfällen“ erlaubt (Gemeinsamer Bundesausschuss 2016; S1Leitlinie Insomnie 2012). Dennoch findet sich im klinischen Alltag oft ein Phänomen, das dem deutschen Gesundheitssystem eigen ist: Kassenärztlich versicherte Patienten erhalten Hypnotika auf Privatrezept. Die Patienten sind somit zum Kauf berechtigt, tragen aber die vollständigen Kosten, da diese Rezepte weder bei den privaten noch den gesetzlichen Kassen eingereicht werden. Zwar ist die Zahl der Hypnotikaverordnungen zu Lasten der gesetzlichen Krankenkassen seit 1992 um fast 80\% rückläufig (Lohse und MüllerOerlinghausen 2016), es muss aber vermutet werden, dass dieser Rückgang zum Teil durch Privatverordnungen zu erklären ist (Hoffmann et al. 2014).

Warum Ärzte, insbesondere niedergelassene Allgemeinmediziner, sich für diese Art der Verschreibung entscheiden, ist zum jetzigen Zeitpunkt nicht ausreichend erforscht.

${ }^{1}$ In der hier vorgestellten Arbeit wird für eine bessere Lesbarkeit die männliche Form verwendet, wobei immer beide Geschlechter gemeint sind. Geht es nur um ein Geschlecht, wird dies im Text explizit erwähnt. 


\section{Stand der Forschung}

\subsection{Verfügbare Benzodiazepine und Benzodiazepin- Rezeptoragonisten}

Das Arzneimittelverzeichnis Rote Liste führt momentan 14 verschiedene Wirkstoffe als Benzodiazepine, verfügbar in insgesamt 40 Präparaten sowie die zwei als „Benzodiazepinähnliche Stoffe" gelisteten Rezeptoragonisten Zolpidem und Zopiclon, verfügbar in jeweils drei Präparaten. Zaleplon, ebenfalls ein Rezeptoragonist, ist separat als Hypnotikum aufgeführt (Rote Liste 2018). Das Deutsche Institut für Medizinische Dokumentation und Information (DIMDI) definiert gemäß der Anatomisch-Therapeutisch-Chemischen (ATC) Klassifikation von 201625 verschiedene Benzodiazepine als Anxiolytika, zwei als Antiepileptika und 13 als Hypnotika und Sedativa. Zu der letzterwähnten Gruppe werden des Weiteren vier „Benzodiazepin-verwandte Mittel“ gerechnet, die neben den oben genannten Rezeptoragonisten auch Eszopiclon enthalten (WIdO/DIMDI 2016).

Benzodiazepine und Rezeptoragonisten könnten sowohl vertragsärztlich, also als reguläres Rezept zu Lasten der gesetzlichen Krankenkassen, als auch auf Privatrezept verschrieben werden. Eine Ausnahme stellt Flunitrazepam dar, das seit dem 1. November 2011 unter das Betäubungsmittelgesetz fällt (Bundesinstitut für Arzneimittel und Medizinprodukte 2018) - auf Grund des hohen Suchtpotentials, wahrscheinlich bedingt durch einen raschen Wirkungseintritt innerhalb von 15 bis 20 Minuten sowie eine hohe Rezeptoraffinität und eine im Vergleich zu anderen Benzodiazepinen stärker sedierende und anxiolytische Wirkung (ratiopharm GmbH 2014; Markus 2007; Bundesapothekerkammer (BAK) 2011).

\subsection{Leitlinien- und indikationsgerechter Gebrauch}

Benzodiazepine spielen in der Anästhesie, Intensiv- und Notfallmedizin eine wichtige Rolle. Sie wirken angstlösend, sedierend, muskelentspannend und antikonvulsiv. Als Prämedikation, also zur Beruhigung unmittelbar vor Operationen, oder zur Narkoseeinleitung sind sie Mittel der ersten Wahl. In der Intensivmedizin werden sie zur Therapie des Delirs oder zur Analgosedierung verwendet, wobei sie keine analgetische Potenz besitzen und hierzu mit Schmerzmitteln kombiniert werden müssen (Schmidt 2007). Als Notfallmedikament dienen sie auch zur Durchbrechung eines epileptischen Anfalls (Erbguth 2015). In der Inneren Medizin werden sie zur Sedierung bei ambulanten Eingriffen, wie Magen-und Darmspiegelungen genutzt (S3-Leitlinie Sedierung in der gastrointestinalen Endoskopie 2014). Und im Bereich der Psychiatrie können sie bei akuten und schweren Angst- und Panikattacken verabreicht werden, wobei eine medikamentöse Therapie in den meisten Fällen nicht notwendig ist (S3-Leitlinie Behandlung von Angststörungen 2014). Kommt es nicht zu 
einem Sturz, einem sonstigen Unfall oder einer seltenen paradoxen Reaktion, ist die einmalige oder kurzfristige Einnahme von Benzodiazepinen in der Regel unproblematisch, da diese Medikament nur in sehr hohen Dosierungen zu einer Atemdepression und lebensbedrohlichen Intoxikation führen. Dies ist jedoch durchaus möglich, insbesondere in Kombination mit Alkohol (Schmidt 2007). Bei längerem Gebrauch kumulieren aber das sporadische Unfallrisiko sowie das Medikament im Körper selbst. Daher kommen die unten näher beschriebenen Nebenwirkungen vorwiegend dann zum Tragen. Aus diesem Grund sollen Patienten laut der S3-Leitlinie ,Angststörungen` Benzodiazepine nicht als Dauermedikament angeboten bekommen und „nur in Ausnahmefällen (z. B. schwere kardiale Erkrankungen, Kontraindikationen für Standardmedikamente, Suizidalität) (...) unter sorgfältiger Risiko-Nutzen-Abwägung zeitlich befristet" anwenden (S3-Leitlinie Behandlung von Angststörungen 2014). Und auch die Nationale Versorgungsleitlinie ,Unipolare Depression“ gibt an, dass Benzodiazepine bei suizidgefährdeten Patienten im Rahmen einer Akutbehandlung von bis zu 14 Tagen in Betracht gezogen werden können, dass diese aber ,praktisch keine antidepressive Wirkung“ haben und langfristig zur „Behandlung der Depression nicht zugelassen“ sind (S3-Leitlinie Unipolare Depression 2017). Das Weiteren sind einige - nicht alle - Benzodiazepine, genau wie die Z-Substanzen, als Hypnotikum zugelassen. Wie bereits in der Einleitung erwähnt, sollte die Therapie von Schlafstörungen mit Hilfe dieser Medikamente aber nur bis zu vier Wochen dauern. Eine längere Verschreibung muss vom Arzt begründet und dokumentiert werden (S1-Leitlinie Insomnie 2012; Gemeinsamer Bundesausschuss 2016; S3-Leitlinie Nicht erholsamer Schlaf/Schlafstörungen 2017).

\subsection{Missbrauchs- und Abhängigkeitspotential}

Trotz ihrer häufigen Verwendung sind die Begriffe Medikamentenmissbrauch und Medikamentenabhängigkeit nicht leicht zu definieren. Die Deutsche Hauptstelle für Suchtfragen (Glaeske und Holzbach 2015) verweist auf die ICD-10-Klassifikation der World Health Organization, der wiederum eine Arbeit von Dilling und Freyberger zugrunde liegt (Dilling und Freyberger 2010) und spricht von Medikamentenmissbrauch, wenn „eine Substanz nicht mehr bestimmungsgemäß eingenommen und der Konsum fortgesetzt wird - trotz psychischer, körperlicher oder sozialer Folgeschäden“. Medikamentenabhängigkeit wird gemäß ICD-10 anhand von sechs Kriterien definiert, von denen mindestens drei innerhalb des letzten Jahres erfüllt gewesen sein müssen:1.) ein „starker Wunsch und/oder Zwang, das Medikament zu konsumieren“, 2.) eine „verminderte Kontrollfähigkeit bezüglich des Beginns, der Menge und/oder der Beendigung der Einnahme“, 3.) das Vorhandensein „körperlicher Entzugssymptome“, 4.) eine „Toleranzentwicklung (Wirkverlust) bzw. Dosissteigerung“" 5.) ein „erhöhter Zeitaufwand, um die Substanz zu beschaffen oder sich von den Folgen des Konsums zu erholen, verbunden mit der Vernachlässigung anderer Interessen“ und 6.) ein „fortgesetzter Konsum trotz Folgeschäden“ (World Health Organization 1992). Bezüglich der Benzodiazepine kann zudem eine primäre Hochdosis- von einer pri- 
mären Niedrigdosis- und Sekundärabhängigkeit unterschieden werden. Die primäre Hochdosisabhängigkeit ist im Vergleich zur Niedrigdosisabhängigkeit selten, äußert sich in einer starken physischen und psychischen Abhängigkeit und geht mit extremen Dosissteigerungen einher. Viel häufiger ist die primäre Niedrigdosisabhängigkeit, bei der Betroffene über einen langen Zeitraum therapeutische Dosen einnehmen und die Dosis nicht gesteigert wird. Beim abrupten Absetzen des Medikaments kommt es aber ebenfalls zu Entzugssymptomen. Eine sekundäre Abhängigkeit entwickelt sich im Zuge einer anderen bereits vorhandenen Abhängigkeit, z. B. eines Alkoholabusus (Deutsche Hauptstelle für Suchtfragen e.V. 2018a).Ungeachtet dieser Definitionen ist es im Einzelfall oft schwer zu beurteilen, an welcher Stelle der Gebrauch trotz Risiken aufhört und der Missbrauch beginnt zumal die Grauzone dieses Übergangs von Arzt und Patient möglicherweise unterschiedlich beurteilt wird.

Das Problem der Toleranz- und Abhängigkeitsentwicklung bei Benzodiazepinverwendung ist schon über 50 Jahre bekannt und vielfach beschrieben (Binder et al. 1984; MüllerOerlinghausen 1986; Lader 1998; Lader 2011; Hollister et al. 1961). Die im Vergleich neueren Benzodiazepin-Rezeptoragonisten galten nach ihrer Einführung in den 1990er Jahren diesbezüglich zunächst als unbedenklicher und werden auch heute noch von vielen Ärzten als nebenwirkungsärmer eingeschätzt (Hoffmann 2013a), obwohl diese Annahme nicht wissenschaftlich belegt ist (Nowell et al. 1997). Dies ist nicht zuletzt der Werbung der Pharmaindustrie geschuldet. So weist die Firma Sanofi auf ihrer Internetseite zwar auf die mögliche „Entwicklung von psychischer und physischer Abhängigkeit“ hin, stellt aber gleichzeitig fest, dass Stilnox® (Zolpidem) „ein geringeres Missbrauchs- oder Abhängigkeitspotential als Medikamente aus der Gruppe der Benzodiazepine“ hat und, dass „während einer Behandlung mit Stilnox ${ }^{\circledR}$ (Zolpidem) von bis zu 4 Wochen bisher keine ausgeprägte Toleranz gegenüber Zolpidem aufgetreten“ ist (Stilnox 2018). Dabei wurden benzodiazepintypische Entzugserscheinungen wie Tachykardien, starkes Schwitzen, Unruhe und Angstzustände - bis hin zu Krampfanfällen - auch nach der Einnahme von ZSubstanzen beschrieben (Weaver 2015; Ströhle et al. 1999; Cimolai 2007); und Patienten berichteten keinen Unterschied zwischen beiden Stoffgruppen bezüglich Wirksamkeit und Nebenwirkungen (Siriwardena et al. 2008). Des Weiteren konnten Hoffmann et al. mit Hilfe der Daten einer gesetzlichen Krankenkasse mit bundesweit 1,47 Mio. Versicherten zeigen, dass fast 7000 Personen im zweiten Halbjahr 2004 eine Z-Substanz verschrieben bekamen, und dass hiervon 7,2\% (501 von 6959 Personen) einen hohen Verbrauch aufwiesen (Hoffmann et al. 2008). Üblicherweise entspricht eine DDD der mittleren täglichen Erhaltungsdosis eines Wirkstoffes bei dessen Hauptindikation für Erwachsene (Fricke et al. 2017). Da aber Z-Substanzen zur kurzzeitigen Therapie von Schlafstörungen verwendet werden sollten, definierten Hoffmann et al. Hochverbrauch als 180 DDD und mehr pro Person im Studienzeitraum, also im Schnitt mindestens 1 DDD pro Tag über sechs Monate. Diese Versichertengruppe suchte im Schnitt drei Ärzte auf, um ein Rezept zu erhalten, hatte ein mittleres Alter von 60 Jahren und nahm mehr Medikamente ein als Personen mit 
einem niedrigeren Verbrauch. Einige der verordnenden Ärzte verschrieben dem jeweiligen Patienten ausschließlich eine Z-Substanz, aber keine weiteren Medikamente, was gegen eine ganzheitliche und umfassende Betreuung spricht (Doktor-Hopping). Die Betroffenen lösten die Rezepte teilweise in mehreren Apotheken ein. Dieses Verhalten wurde von den Autoren als „Craving im Sinne einer psychischen Abhängigkeit“ gewertet (Hoffmann et al. 2008).

Diese Erkenntnisse finden aber erst nach und nach Einzug in Leit- und Richtlinien. Während die als Beers-Kriterien bekannten Empfehlungen der American Geriatrics Society (AGS) im Jahr 2012 die Verwendung von Z-Substanzen zuließen, d. h. sich lediglich gegen eine Verordnung von mehr als 90 Tagen aussprachen, heißt es im Statement der AGS Arbeitsgruppe Choosing Wisely von 2013, dass sowohl Benzodiazepine als auch ZSubstanzen bei älteren Menschen „sparsam“ verwendet werden sollten, ohne dass dies bzgl. Menge und Dauer näher spezifiziert ist. Im Update der Beers-Kriterien von 2015 wird schließlich geraten, diese Medikamente bei Patienten über 65 unabhängig von der Einnahmedauer strikt zu vermeiden (AGS Choosing Wisely Workgroup 2013; American Geriatrics Society 2012, 2015). Basierend auf den Daten der über 9,1 Millionen Menschen, die während des Jahres 2012 bei der BARMER GEK versichert waren, schätzen die Autoren des BARMER GEK Arzneimittelreport 2013, dass 30 bis 50 Prozent der Schlafmittel nicht wegen akuter medizinischer Probleme verwendet werden, sondern vermuten, dass diese langfristig - oft zur Suchterhaltung oder zur Vermeidung von Entzugserscheinungen verschrieben werden (Glaeske und Schulze 2013). Zwei Drittel aller Verordnungen weisen einen längeren Zeitraum als 8-14 Tage auf (Glaeske 2017) und laut Holzbach et al. münden mindestens 35\% in einer Langzeitverschreibung (Holzbach et al. 2010). Dies ist problematisch, da neben einer Toleranz- und Abhängigkeitsentwicklung weitere Nebenwirkungen, wie Tagesmüdigkeit, gestörte Koordination und Stürze (Hill und Wee 2012; Xing et al. 2014) auftreten können. Zwar ist auch bei einmaliger oder kurzfristiger Einnahme eine körperliche und geistige Beeinträchtigung möglich. Jedoch weisen mehrere Studien, u. a. eine Fall-Kontroll-Studie, darauf hin, dass diese negativen Effekte möglicherweise persistieren und dass die dauerhafte Verwendung von Benzodiazepinen eine Demenzentwicklung fördert (Rummans et al. 1993; Markota et al. 2016; Puustinen et al. 2012)

Insgesamt gehören Hypnotika zu den am häufigsten missbräuchlich angewendeten Arzneistoffen. Die Deutsche Hauptstelle für Suchtfragen e.V. gibt an, dass in Deutschland 1,4 Millionen medikamentenabhängige Menschen leben (Deutsche Hauptstelle für Suchtfragen e.V. 2018b). Laut des BARMER GEK Arzneimittelreportes 2013 gibt sogar bis zu 1,9 Millionen; 1,2 Millionen davon (63 Prozent) verwenden ein Benzodiazepin-haltiges Mittel (Glaeske und Schulze 2013). Ähnlich schätzt die Bundesärztekammer die Gesamtzahl der Abhängigen (1,4 bis 1,9 Millionen), gibt jedoch an, dass Benzodiazepine sogar in 80 Prozent der Fälle eine Rolle spielen (Bühring 2007). Weitere 1,7 Millionen Menschen gelten als mittel- bis hochgradig abhängigkeitsgefährdet (Apothekenkammer Hamburg 2011). Damit 
ist in Deutschland nicht etwa die Alkohol-, sondern die Medikamentenabhängigkeit - nach der Tabakabhängigkeit - das zweitgrößte Suchtproblem (Glaeske 2017).

Betroffen sind häufig Personen über 65 Jahre, darunter zwei Drittel Frauen (Glaeske und Schulze 2013) Dies deckt sich auch mit den Ergebnissen einer von der Arzneimittelkommission der Deutschen Apotheken durchgeführten Umfrage. Mehr als die Hälfte der teilnehmenden Apotheken gaben darüber hinaus an, Hinweise auf missbräuchliche Anwendung von Hypnotika zu haben, wie etwa häufige Verordnungen oder Verordnungen großer Menge - teilweise von verschiedenen Ärzten -, das Verlangen des Medikamentes ohne Rezept oder die Vorlage gefälschter Rezepte (Diemert et al. 2013). Kapil et al. konnten mit Hilfe eines Online-Fragebogens, der durch ein Marktforschungsinstitut an Briten im Alter von 16-59 Jahre gesendet wurde, zeigen, dass 26,1\% (392 von 1500) der (hinsichtlich Selektionskriterien und Response nicht näher beschriebenen) Studienpopulation Benzodiazepine oder Z-Substanzen eingenommen hatte oder noch einnahm. Von diesen gaben circa 30\% (116 von 392) einen Missbrauch des jeweiligen Medikaments zu; als Gründe hierfür wurden u. a. Stressbewältigung $(37,1 \%, n=43)$ oder high werden $(31 \%, n=36)$ genannt. Fast $40 \%$ der 116 Personen hatten das Medikament von Freunden oder Familienmitgliedern bekommen, die meisten $(55,2 \%)$ erhielten es jedoch mittels eines Rezeptes vom Arzt. Andere Quellen waren das Internet oder Dealer (Kapil et al. 2014).

\subsection{Verordnungsdaten}

Als Schlafmittel wurden laut des BARMER GEK Arzneimittelreportes im Jahr 2013 (mit Schwerpunkt Hypnotika) vor allem Zolpidem (als Stilnox ${ }^{\circledR}$ und Generika), ZopiclonGenerika, Brotizolam in Form von Lendormin $\AA$, Lormetazepam in Form von Noctamid ${ }^{\circledR}$, Nitrazepam als Radedorm ${ }^{\circledR}$, Flunitrazepam (als Rohypnol ${ }^{\circledR}$ und Generika) und Temazepam als Planum ${ }^{\circledR}$ verordnet. Die meist verwendeten Tranquilizer waren Diazepam (als Generika und Faustan $\left.{ }^{\circledR}\right)$, Lorazepam (als Generika und Tavor $\left.{ }^{\circledR}\right)$, Bromazepam (als Generika, Lexotanil ${ }^{\circledR}$ und Normoc ${ }^{\circledR}$ ), Oxazepam (als Generika und Adumbran ${ }^{\circledR}$ ) und Clorazepat in Form von Tranxilium ${ }^{\circledR}$ (Glaeske und Schulze 2013).

Wie die Zahlen des Arzneiverordnungsreports 2016 zeigen, verordnet der einzelne Hausarzt oder hausärztlich tätige Internist pro Jahr weitaus weniger Psycholeptika, zu denen auch die Benzodiazepine und Z-Substanzen gehören (ATC-Code N05), als ein Nervenarzt ${ }^{2}$, Neurologe oder Psychiater; nämlich 3.916,6 bzw. 3.395,1 DDD verglichen mit 51.077,1, 14.139,5 und 36.481,2 DDD. Da aber 40.461 Hausärzte und 14.713 hausärztlich tätige Internisten nur 2331 Nervenärzten, 1912 Neurologen und 2104 Psychiater gegenüberstehen, werden die meisten Psycholeptika durch die Gruppe der Hausärzte verschrieben (158.469.553 DDD pro Jahr), gefolgt von der Gruppe der Nervenärzte (119.060.720 DDD

${ }^{2}$ Laut Auskunft der Bundesärztekammer bezieht sich der Begriff Nervenarzt auf eine vor 2003 erworbene Facharztbezeichnung. Diese ist heute durch den Facharzt für Neurologie und den Facharzt für Psychiatrie und Psychotherapie ersetzt worden. 
pro Jahr). An dritter Stelle findet sich die Gruppe der „Sonstigen“ Ärzte, zu denen u. a. Kinderneuropsychiater, Psychologische Psychotherapeuten und Kinder- und Jugendpsychotherapeuten zählen: 14.547 Ärzte verschreiben im Schnitt je 8.059,6 DDD pro Jahr und somit als Gruppe 117.243.001 DDD (Schaufler und Telschow 2016). Auch international werden die meisten Hypnotika von Hausärzten verordnet (Markota et al. 2016; Weisberg et al. 2007; Donoghue und Lader 2010). Leider ist nicht bekannt, in welcher Fachgruppe wie viele leitliniengerechte bzw. nicht-leitliniengerechte Verordnungen getätigt wurden.

\subsection{Einstellung von Ärzten gegenüber Hypnotika}

Eine initiale Pubmed-Suche zu den Begriffen „benzodiazepine AND prescribing AND attitude“ erbrachte insgesamt 52 Treffer, von denen sich 32 nicht im engeren Sinne mit der Perspektive des Arztes in Bezug auf Hypnotikaverordnungen beschäftigten. Mit Hilfe der Suchwörter „Z-drugs AND prescribing AND attitude“ fanden sich fünf Artikel; vier davon waren bereits in den 20 übrigbleibenden Artikeln der ersten Suche enthalten, der fünfte stellte die Sicht von Patienten dar.

Tabelle 1: Darstellung der Medical Subject Headings (MeSH)-Suche in Pubmed

\begin{tabular}{|c|c|c|c|c|c|c|c|}
\hline \multirow[t]{9}{*}{$\mathrm{MeSH}$} & $\begin{array}{l}\text { Attitude } \\
\text { of Health } \\
\text { Personnel }\end{array}$ & $\begin{array}{l}\text { Hypnotics } \\
\text { and } \\
\text { Sedatives }\end{array}$ & $\begin{array}{c}\text { Physician's } \\
\text { Practice } \\
\text { Patterns }\end{array}$ & $\begin{array}{c}\text { Health } \\
\text { Expenditures }\end{array}$ & $\begin{array}{c}\text { Physicians, } \\
\text { Family }\end{array}$ & Ergebnis & Relevant \\
\hline & $\mathrm{x}$ & $\mathrm{x}$ & & & & 191 & \\
\hline & $\mathrm{x}$ & $\mathrm{x}$ & $\mathrm{x}$ & & & 28 & 8 \\
\hline & $\mathrm{x}$ & $\mathrm{x}$ & & $\mathrm{x}$ & & 0 & \\
\hline & $x$ & & & $x$ & & 181 & \\
\hline & $\mathrm{x}$ & & $\mathrm{x}$ & $\mathrm{x}$ & & 12 & 1 \\
\hline & & $x$ & & & $x$ & 17 & 4 \\
\hline & & $x$ & $\mathrm{x}$ & & $\mathrm{x}$ & 4 & 3 \\
\hline & & & & $\mathrm{x}$ & $\mathrm{x}$ & 48 & 0 \\
\hline
\end{tabular}

Im Folgenden wurden die Medical Subject Headings (MeSH)-Stichwörter "Attitude of Health Personnel"[MeSH], "Hypnotics and Sedatives"[MeSH], "Physician's Practice Patterns"[MeSH], Health Expenditures"[MeSH] und "Physicians, Family"[MeSH] miteinander kombiniert. Das Ergebnis dieser Suche ist zur besseren Übersicht in Tabelle 1 dargestellt. Zu beachten ist, dass „Health Expenditures“ $[\mathrm{MeSH}]$ die Begriffe „Out-of-pocket Expenditure” und „Out-of-pocket Payment” miteinschließt; „Physician's Practice Patterns“[MeSH] und „Physician's Prescribing Patterns“ $[\mathrm{MeSH}]$ konnten synonym verwendet werden.Neun 
dieser 16 Artikel waren bereits durch die initiale Suche gefunden worden, so dass insgesamt 27 Pubmed-Artikel als relevant identifiziert werden konnten.

Mit Hilfe von EMBASE wurde zunächst nach den gleichen Stichwörtern „benzodiazepine AND prescribing AND attitude“" gesucht. Anschließend erfolgte eine einschränkende Suche, bei der „attitude“ durch „Attitude of Health Personnel“ ersetzt wurden. Des Weiteren wurde „Attitude of Health Personnel“ mit den gleichen vier Begriffen kombiniert, die bei der Pubmed MeSH-Suche verwendet wurden. Elf weitere relevante Artikel konnten so gefunden werden. Als dritte Datenbank wurde CC MED verwendet. Die Suche erfolgte, wie zuvor, durch Kombination der Stichworte. Es wurden allerdings keine neuen relevanten Artikel gefunden.

Wie aus Abbildung $1 \mathrm{zu}$ entnehmen ist, stellen alle Studien, bis auf eine Ausnahme (Sirdifield et al. 2013), Primärdaten dar. Vier der Artikel waren in Dänisch (Troest 1993) oder Norwegisch (Albretsen 1991; Kristensen und Fjermestad 1991; Bachmann et al. 2008) verfasst. Für einen dieser Artikel war kein englisches Abstract verfügbar (Albretsen 1991); in einem weiteren Artikel fand sich keine Angabe zur Methode im Abstract (Troest 1993). Zwei kanadische Studien gaben die Einstellung der Ärzte gegenüber Hypnotika nur indirekt wider, indem sie Krankenkassendaten mit demographischen Informationen in Zusammenhang brachten (Davidson et al. 1994; Monette et al. 1997).

Die Studien, die die Meinungen der Ärzte direkt erfassten, arbeiteten meisten mit Fragebögen (21 von 33), welche entweder per Post zugeschickt und von den Teilnehmern selbststätig ausgefüllt oder von einem Mitglied des Forscherteams per Telefon oder bei einem persönlichen Treffen mit dem jeweiligen Arzt genutzt wurden. Von den 21 Arbeiten stammen 16 aus Europa (davon drei aus Deutschland: Hoffmann et al. 2014; Hoffmann 2013a; Hoffmann 2013b), drei aus dem US-amerikanischen und jeweils ein Artikel aus dem afrikanischen und asiatischen Raum.

Von den zehn Studien, in denen Ärzte interviewt wurden, stammt ein Artikel aus Australien, einer aus den USA und acht aus Europa - jedoch nur einer aus Deutschland (Flick et al. 2012). Einer dieser zehn Artikel erfasst die Meinungen und Erfahrungen von Ärzten, die ausschließlich stationäre psychiatrische Patienten behandeln (Haw und Stubbs 2007). In den übrigen neun Studien wurden Interviews mit niedergelassenen Allgemeinmedizinern geführt. Teilweise wurde diese durch Interviews mit Patienten (Iliffe et al. 2004), Apothekern (Cheung et al. 2014) und Ärzten in Weiterbildung (Deans und Skinner 1992) ergänzt. In der deutschen Interviewstudie sprachen Flick et al. 2012 mit 20 Ärzten, die in Altersheimen tätig waren. Diese besaßen die Facharztbezeichnung für Allgemeinmedizin, Innere Medizin oder Psychiatrie - vier der 20 hatten eine geriatrische Zusatzqualifikation erworben - und waren entweder in dem jeweiligen Altersheim angestellt $(n=11)$ oder betreuten dieses im Rahmen ihrer selbststätigen niedergelassenen Tätigkeit ( $\mathrm{n}=9$ ) (Flick et al. 2012). Die Anstellung eines Arztes in einem Heim ist jedoch ein in Deutschland nicht übliches Modell, daher muss hier von einer selektiven Stichprobe ausgegangen werden. 
Zusätzlich zu den Quellenangaben im Literaturverzeichnis findet sich die Liste aller 38 Artikel der Literaturrecherche am Ende der Arbeit (s. Anhang 8.1).

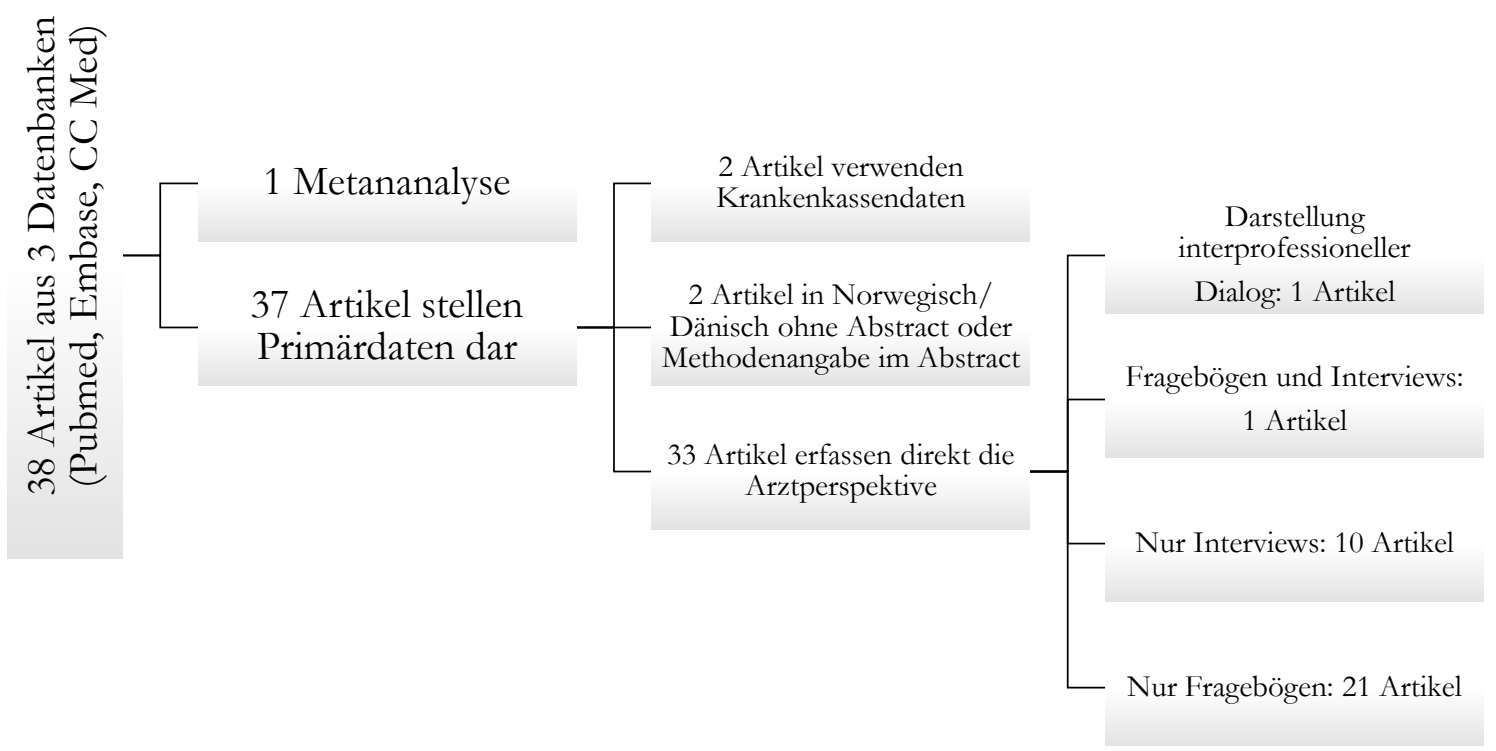

Abbildung 1: Übersichtsdarstellung der relevanten Artikel in 2.5

Im Folgenden werden die Ergebnisse der Literaturrecherche zu Einstellungen von Ärzten gegenüber Hypnotika thematisch zusammengefasst dargestellt. Hierbei sind sowohl die Metaanalyse (Sirdifield et al. 2013) und die zwei Krankenkassendaten-verwendenden Artikel (Monette et al. 1997; Davidson et al. 1994) als auch die weiteren 33 Primärarbeiten zur Arztperspektive berücksichtigt. Teilweise werden diese durch weitere Artikel ergänzt, die im Laufe des Projektes hinzukamen und sich als themenrelevant herausstellten. Ausgeschlossen wurden ein norwegischer (Albretsen 1991) und ein dänischer (Troest 1993) Artikel.

Auffallend ist, das obwohl nach „Attitude of Health Personnel“ gesucht wurde, oft über soziodemographische Eigenschaften der verschreibenden Ärzte als auch der Patienten berichtet wird - und dies nicht nur in den Arbeiten von Monette und Davidson (Davidson et al. 1994; Monette et al. 1997). So soll zunächst dargestellt werden, ob und wie sich Geschlecht, Alter und Ausbildung eines Arztes auf sein Verschreibungsverhalten bezüglich Hypnotika auswirken. Anschließend werden die Ergebnisse von Arbeiten zusammengefasst, die sich mit dem stereotypischen Patienten, also dem BenzoZ-Konsumenten beschäftigen. Mit Hinblick auf die Fragestellung dieses Projektes wird des Weitern beschrieben, wie Ärzte nach dem heutigen Stand der Forschung die Indikation für ein Benzodiazepin oder eine Z-Substanz einschätzen, wie sie die Risiken und Nebenwirkungen dieser Medikamente sowie alternative Behandlungsoptionen wahrnehmen und wie sie ihre eigene Verantwortung, aber auch die Verantwortung des Patienten im Umgang mit Schlaf- und Beruhigungsmitteln bewerten. 


\subsubsection{Verschreibungsverhalten in Abhängigkeit vom Geschlechts des Arztes}

Die Auswertung der kanadischen Krankenkassendaten in den 90er Jahren zeigte, dass Ärzte, die Benzodiazepine über dem aus den Verordnungsdaten ermittelten Durchschnitt verschrieben, im Schnitt mehr Praxistage hatten, mehr Patienten pro Tag sahen und mehr Leistungen bei den Krankenkassen abrechneten (Davidson et al. 1994) sowie ihr Medizinstudium vor 1979 abgeschlossen und in ihrer alltäglichen Arbeit keine universitäre Anbindung hatten (Monette et al. 1997). In beiden Studien verordneten Männer mehr Benzodiazepine als Frauen, wobei deutlich weniger Ärztinnen als Ärzte eingeschlossen wurden: $13.5 \%$ vs. $86.5 \%$ (Monette et al. 1997) und 17\% vs. $83 \%$ (Davidson et al. 1994) und nicht angegeben wird, ob es hierbei um die Basisverteilung oder unterproportional wenig Frauen handelt. Eine Untersuchung von 2003, bei der Faktoren, die mit einem hohen Verschreibungsvolumen von Benzodiazepinen assoziiert waren, mittels eines Fragebogens erfasst wurden, kam bezüglich des Geschlechts zu einem ähnlichen Ergebnis (Bjørner und Laerum 2003). Jedoch waren auch hier zwei Drittel der 1018 Teilnehmer männlich. Des Weiteren war es entscheidend, ob Ärzte die Verschreibung von Schlaf- und Beruhigungsmitteln als schwierig wahrnahmen, da ihre Verordnung im Widerspruch zu bestehenden Leitlinien stand, ihren Patienten erlaubten, die Verschreibungsentscheidung zu beeinflussen oder Rezepte ohne direkten Arztkontakt herausgaben. Der Geschlechterunterschied zeigte sich aber auch in Interviewstudien, in denen im Vorfeld „Viel- und Wenig-Verschreiber“ basierend auf Apothekendaten identifiziert wurden: In einer norwegischen Studie von 1997 waren alle 18 „Viel-Verschreiber“ (über 4000 DDD Benzodiazepine und schwache Opiate pro Monat) männlich, hingegen die Gruppe der „Wenig-Verschreiber“ aus sechs Männern und vier Frauen bestand (Dybwad et al. 1997). Nach dem Vorbild dieser Studie interviewten Šubelj et al. 2010 zehn Ärzte; die fünf „Viel-Verschreiber“ waren ebenfalls männlich. In der Gruppe der „Wenig-Verschreiber“ befanden sich 2 Frauen. Allerdings war die Gruppe der „Viel-Verschreiber“ im Schnitt zehn Jahre älter und besaß nur zu 50\% im Vergleich zu 80\% eine Facharztweiterbildung, so dass hier möglicherweise ein Confounding-Effekt vorliegt (Subelj et al. 2010). In einer älteren Arbeit spielt das Geschlecht des Verordnenden keine Rolle - sehr wohl aber das Geschlecht des Patienten (Heiman und Wood 1981). An anderer Stelle scheint beides von Bedeutung; so erhielten Patientinnen mehr psychotrope Medikamente von Ärztinnen (Morabia et al. 1992).

\subsubsection{Verschreibungsverhalten in Abhängigkeit vom Alter und der Ausbildung des Arztes}

Neben dem Geschlecht und Alter des Patienten sowie dem Geschlecht des Arztes scheint auch das Alter des Arztes bzw. die Zeit seit dem Hochschulabschluss sowie dessen Weiterbildung eine Rolle zu spielen. Auch wenn sich in einer Studie von 1996 kein Zusammenhang zwischen Letzterem und der Häufigkeit von Benzodiazepin-Verschreibungen zeigte (Boixet et al. 1996), wird an anderer Stelle berichtet, dass Ärzte mit mehr akademischem Training die Effektivität von Beruhigungsmitteln zur Langzeittherapie geringer einschätz- 
ten, da sie von einen Wirkverlust bzw. Gewöhnungseffekt ausgingen und die Präparate dementsprechend weniger verwendeten (Chambers et al. 1983). Ärzte, die häufig Benzodiazepine verordneten, waren im Schnitt älter als ihre Kollegen, die weniger verschrieben und besaßen in 50\% der Fälle keine Facharztqualifikation (Subelj et al. 2010; Dybwad et al. 1997). Vermutlich ist dieses Phänomen bedingt durch die sich verändernde Studienlage bezüglich der Schlaf- und Beruhigungsmittel. So stellten Chambers et al. 1983 fest, dass Ärzte, die in den letzten Jahren ihre Ausbildung abgeschlossen hatten, Angststörungen häufiger adäquat diagnostizierten und behandelten und weniger häufig allein auf pharmakologische Therapien setzen (Chambers et al. 1983). Und auch in einer Studie von 1992 zeigten sich Weiterbildungsassistenten vorsichtiger im Umgang mit Benzodiazepinen als erfahrene Ärzte (Deans und Skinner 1992). Ein unterschiedliches Antwortverhalten von über 45 Jahre alten und jüngeren Ärzten konnten auch Hamilton et al. beobachten. Jüngere Kollegen stimmten beispielsweise dem Satz zu „Benzodiazepin-Überverordnungen könnten reduziert werden, wenn mehr psychologische Angebote als Alternative verfügbar wären“. Es ergab sich allerdings in dieser Studie kein eindeutiger altersassoziierter Trend für oder gegen Benzodiazepine (Hamilton et al. 1990). An anderer Stelle wird berichtet, dass jüngere Ärzte Benzodiazepinen kritischer gegenüber stehen als ältere Kollegen und seltener Rezepte ausstellen (Anthierens et al. 2010). In dieser Umfrage unter 948 belgischen Allgemeinmedizinern zeigten sich fast 40\% - überwiegend Ärzte über 55 Jahre - nicht wesentlich besorgt über die Risiken von Benzodiazepin-Verschreibungen; 17\% war die Problematik bewusst, jedoch empfanden sie die Nutzung von nicht-medikamentösen Therapien als nicht zu ihrem Aufgabenfeld gehörend. Der Großteil der Befragten sah die Verwendung von nicht-medikamentösen Therapien als eine bessere Lösung an, begegnete jedoch Hürden bei der Umsetzung (Anthierens et al. 2010) Allerdings kommen Siriwardena et al. im Gegensatz zu den oben zitierten Untersuchungen zu dem Schluss, dass Ärzte unter 34 Jahre eine eher positive Einstellung zu Benzodiazepinen und Z-Substanzen haben (Siriwardena et al. 2010).

Ein weiterer altersbezogener Unterschied zeigte sich in einer Telefonumfrage unter Allgemeinärzten bezüglich des Eigengebrauchs von Schlaf- und Beruhigungsmitteln. Insgesamt war dieser höher als in der Allgemeinbevölkerung - und zwar alters- und geschlechtsunabhängig. Aber bei Ärzten über 48 Jahre war der Gebrauch signifikant assoziiert mit der Arbeitsbelastung und -unzufriedenheit sowie einem Mangel an Weiterbildung (Verger et al. 2004)

\subsubsection{Der typische Patient}

In der US-amerikanischen Studie von Heiman und Wood war es wahrscheinlicher, dass ein männlicher Patient ein Benzodiazepin erhielt und dass Benzodiazepin-Verschreibungen bei Frauen bei nachlassender Chronizität zunahmen, was von den Autoren selbst als entgegen der Studienlage dargestellt wurde (Heiman und Wood 1981). Die Chronizität ergab sich aus unterschiedlichen Fallvignetten mit unterschiedlichen Chronizitäts-Scores; die Teilnehmer 
wurden gebeten jeweils zu bewerten, für wie indiziert sie eine BZD-Verordnung hielten. Nach verfügbarer Datenlage zu Verordnungen erhalten mehr Frauen als Männer, mehr Ältere als jüngere Patienten und damit auch mehr chronisch Kranke Benzodiazepine (Subelj et al. 2010; Boixet et al. 1996; Cheung et al. 2014). Dies deckt sich mit den Ergebnissen der in Abbildung 1 erwähnten Metaanalyse von 2013. Sirdifield et al. fassten acht Artikel aus sieben westlich geprägten Ländern zusammen (einschließlich zwei bereits erwähnter Studien Subelj et al. 2010; Dybwad et al. 1997), die sich mit dem Thema Erfahrungen und Wahrnehmungen von Allgemeinärzten bezüglich Benzodiazepin-Verschreibungen beschäftigten (Sirdifield et al. 2013). Dabei charakterisierten sie, insbesondere auf der Studie von Rogers et al. basierend, den Patiententypus, der ein Schlafmittelrezept „verdient“ (Rogers et al. 2007). Dieser ist weiblich, älter, multimorbide, nimmt seit längerer Zeit Schlafmittel, trauert oder ist unheilbar krank. Im Umgang mit dieser (prototypischen) Patientin wird Empathie dann oft durch ein Rezept ausgedrückt. Alkohol- oder drogenabhängige Patienten erhielten jedoch weniger häufig Hypnotikarezepte, da die Ärzte zum einen befürchteten durch eine Verschreibung die Sucht des Patienten oder den Schwarzmarkthandel zu unterstützen, zum andern diese als „Ressourcenverschwendung“ ansahen. Ob sich der Ausdruck auf die Zeit des Arztes, den Wert des Medikamentes oder den erwarteten geringen Behandlungserfolg der Schlafstörung bezieht, wird in der Arbeit von Rogers et al. nicht näher ausgeführt. Ältere Menschen, die kein klassisches Suchtverhalten, wie das Verlangen nach höheren Dosen, zeigen, werden hingegen als nicht abhängig wahrgenommenen und eine stabile Dosis wird häufig als sichere Dosis bewertet (Cook et al. 2007), auch wenn dies, wie unter 2.3 beschrieben, einer primären Niedrigdosisabhängigkeit entspräche (Deutsche Hauptstelle für Suchtfragen e.V. 2018a). Mit Blick auf dieses Phänomen - einige Patienten erwecken mehr Sympathie als andere - ist vielleicht auch die Anmerkung von Heiman und Wood zu sehen, die bezüglich ihrer Ergebnisse als möglichen Confounder angaben, dass keine der teilnehmenden Patientinnen eine Arbeit hatte, jedoch alle männlichen Patienten, die an der Studie teilnahmen (Heiman und Wood 1981).

\subsection{4 Ärztliche Einschätzungen zur BenzoZ-Indikation}

Bjørner und Kjølsrød identifizierten in ihrer Interviewstudie acht Modelle oder typische Situationen, in denen Benzodiazepine verschrieben wurden (Bjørner und Kjølsrød 2002). Neben Angst- und Schlafstörungen zählten auch akute und chronische Schmerzen unklarer Genese zu den häufigen Gründen. Des Weiteren erhielten Patienten in akuten Krisensituationen - ohne, dass explizit die Dauer der Anwendung diskutiert wurde - oder mit schweren somatischen Erkrankungen ein Rezept. Letzteres insbesondere, weil die Fähigkeit des Patienten, seine Erkrankung (ohne Beruhigungsmittel) zu bewältigen, als gering eingeschätzt wurde. Ähnlich wie in der Arbeit von Rogers et al. (Rogers et al. 2007), taucht auch hier der Begriff des Patienten auf, der ein Benzodiazepin „,verdient“ hat. Dieser begann laut Bjørner und Kjølsrød die Einnahme oft auf Grund einer Krise, beispielsweise eines Todesfalls, einer Scheidung oder eines arbeitsbezogenen Problems, hat aber große Schwierigkei- 
ten sein Leben zu ordnen und wird so zum Langzeitverwender. Eine weitere typische Situation ist der Umgang mit Patienten, bei denen jegliche Hoffnung auf einen Entzug aufgegeben wurde. Diese sind oft alkohol- und drogenabhängig, beziehen Sozialleistungen und sind arbeitslos. Als letztes wurde die Betreuung von Patienten genannt, die zwar Benzodiazepine einnehmen, jedoch über ausreichende Ressourcen verfügen, einer Diskussion über die Weiterführung und einen Entzug standzuhalten. Hier äußerten die Befragten die größte Bereitschaft bezüglich eines Absetzversuchs (Bjørner und Kjølsrød 2002).

In der deutschen Interviewstudie identifizierten Flick et al. drei verschiedene Verschreibungstypen (Flick et al. 2012): Die erste Gruppe verschrieb Schlafmittel „auf Anfrage“, weil sie sich unter Druck gesetzt fühlten und Konflikte mit den Patienten, in diesem Fall den Bewohnern des Altersheims, und den Pflegekräften vermeiden wollten. Diesen Ärzten war bewusst, dass Verschreibungen oft keine medizinische, sondern eine „umgebungsbezogene soziale Indikation" hatten, wie z. B. den routinemäßigen Ablauf auf Station nicht zu gefährden. Des Weiteren glaubten die Befragten, dass die Patienten ein „Recht auf Medikamente hätten, um ihre noch verbleibende Lebenszeit zu genießen" und nahmen nichtmedikamentöse Therapien und pflanzliche Mittel bezüglich der Vermeidung von Schlafstörungen als uneffektiv war. Die zweite Gruppe zeigte sich „ambivalent“. Diese Ärzte sahen Hypnotika als letzte Wahl zur Behandlung von Insomnien, konnten alternative Maßnahmen aber oft nicht umsetzen, da Patienten beispielsweise kognitiv nicht in der Lage waren, Schlafhygienemaßnahmen zu befolgen oder „homöopathische“ Mittel nicht von der Krankenkasse finanziert wurden. Diese Gruppe stand der initialen Verordnung sehr kritisch gegenüber, akzeptierte aber den langfristigen Gebrauch. Als letztes Verhaltensmuster zeigte sich die „reflektierte Verordnung“. Diese Ärzte nutzten Hypnotika nur für kurze Zeiträume, beispielsweise einer Krisenintervention, und fühlten sich dafür verantwortlich, ihre Patienten vor einer Abhängigkeit zu bewahren. Ihnen war es wichtig, dass Patienten das Gefühl hatten, dass ihre Schlafprobleme ernst genommen werden - auch wenn keine Medikation erfolgt. Für die Autoren stellten die drei identifizierten Gruppen ein Kontinuum dar, bei dem die Gruppen „auf Anfrage“ und „reflektierte Verordnung“ einen maximalen Kontrast bildeten und die ambivalente Gruppe zwischen diesen beiden Polen lag. Ob Ärzte situativ von einer Gruppe die andere rutschen, wird in dem Artikel nicht diskutiert. Allerdings wird noch einmal darauf hingewiesen, dass sich die Interviews auf Schlafmittel im Allgemeinen und nicht auf einzelne Medikamente bezogen (Flick et al. 2012).

In einer älteren amerikanischen Studie wurden 50 Ärzte, die nach den Daten der Krankenhausapotheke in den letzten 24 Stunden Benzodiazepine verschrieben hatten, per Telefon kontaktiert und nach dem Grund der Verschreibung gefragt. Obwohl BZD in den meisten Fällen (26\%) zur Prämedikation verwendet wurden, waren diese Medikamente in 14\% der Fälle gegen Schmerzen oder in 12\% gegen Übelkeit verordnet worden (Zisselman et al. 1997). Auch wenn die Gabe vor Operationen nach wie vor zu den häufigsten Indikationen gehört, decken sich die zuletzt genannten Gründe nicht mit den heutigen Leitlinien (Vergleiche 2.2). 
Während die Schlafmittelverwendung und -problematik in entwickelten und westlich geprägten Ländern relativ einheitlich zu sein scheint (Sirdifield et al. 2013)und viele frühere BZD-Indikationen dort heute obsolet sind, sollte die Einstellung von Ärzten in Entwicklungsländern möglicherweise vor einem anderen Hintergrund gesehen werden. So richtet sich die Verwendung von Medikamenten eventuell nicht nach strikter Indikationsstellung, sondern nach deren Verfügbarkeit. An einer thailändischen Fragebogen-Studie von 2005 nahmen 55 von 100 eingeladenen Allgemeinmedizinern teil; hiervon nutzten 27\% Benzodiazepine zur Behandlung von Bluthochdruck (Srisurapanont et al. 2005). Und eine Studie aus dem Senegal kommt beispielsweise zu dem Schluss, dass zur Behandlung von isolierten Insomnien anstelle von Prazepam Benzodiazepine mit einer kürzeren Halbwertzeit, wie Flunitrazepam, verwendet werden sollten, welches - wie erwähnt - in Deutschland mittlerweile unter das Betäubungsmittelgesetz fällt (Dièye et al. 2006). Gemeinsamkeiten bestehen darin, dass auch Ärzte in Entwicklungsländern einen Zeit- und Wissensmangel beklagten (Srisurapanont et al. 2005). Bachmann et al. konnten 2008 mit Hilfe eines Fragebogens zeigen, dass nicht nur eine kritischere Einstellung gegenüber Hypnotika, sondern auch adäquates Wissen - auch bezüglich alternativer Therapien - mit weniger Verschreibungen einhergeht (Bachmann et al. 2008).

\subsection{5 Ärztliche Wahrnehmung der Alternativen zu BenzoZ}

Alternative Behandlungsansätze wurden insbesondere als zeitraubend (Anthierens et al. 2010; Bourgeois et al. 2014), aber auch als schwer verfügbar (im Falle einer geplanten Überweisung) und für den Patienten stigmatisierend und kostspielig betrachtet (Sirdifield et al. 2013). Die von Cook at al. befragten Ärzte gaben ebenfalls an, dass auf Grund des Zeitmangels wichtigere medizinische Probleme, wie die Verhinderung von Herzinfarkten und Schlaganfällen, Vorrang hätten (Cook et al. 2007), so dass die Hintergründe der Insomnie, alternative Therapien oder eine bestehende Schlafmittelabhängigkeit nicht angesprochen werden könnten. Hinzu kommt, dass zwei von drei Allgemeinmedizinern ein nicht ausreichendes Wissen auf diesem Feld beklagten (Anthierens et al. 2010), z. B. dass, kognitive Verhaltenstherapie zur Behandlung von Insomnien genutzt werden kann (Everitt et al. 2014). Eine weitere wichtige Hürde bei der Umsetzung nicht-medikamentöser Therapien, ist die Annahme, der Patient sei für solche Behandlungen schwer zu motivieren, da er sich zum einen nicht ändern will (Anthierens et al. 2010) oder Verhaltensänderungen bereits erfolglos probiert hätte und ein Rezept erwarte. Daher wird häufig der Weg des geringsten Widerstandes gewählt. Everitt et al. konnten mit Hilfe von teilstrukturierten Interviews und Fragebögen zeigen, dass bei Schlafproblemen fast alle teilnehmenden Allgemeinmediziner zunächst Schlafhygiene-Beratung anboten. Gleichzeitig wurde diese Intervention von vielen als ineffektiv betrachtet, da Ärzte zum einen den Eindruck hatte, dass Patienten bereits über ausreichendes Wissen zur Schlafhygiene verfügen und Maßnahmen im Vorfeld umgesetzt hätten, zum anderen hatte fast jeder Fünfte den Eindruck, dass das Nicht-Ausstellen eines Rezeptes die Arzt-Patienten-Beziehung gefährden könnte (Everitt et 
al. 2014). Diese Annahmen stimmen aber häufig nicht mit der tatsächlichen Patientenerwartung überein (Brody et al. 1997) und die Bereitschaft der Patienten zu einen Entzug auch nach längerem Benzodiazepingebrauch - wird von Ärzten eher unterschätzt (Morrice und Iliffe 1992). Der erwartete Widerstand der Patienten erwies sich auch als eine - jedoch nicht die einzige - entscheidende Hürde bezüglich des Absetzens von Benzodiazepinen in Pflegeheimen. Neben den Ärzten gaben die Pflegekräfte ebenfalls an, dass alternative Behandlungsansätze zu zeitaufwändig wären. Eine fehlende Motivation der Pflegekräfte wurde allerdings von beiden Berufsgruppen - im Vergleich zum Patientenwiderstand und dem zeitlichen Mehraufwand - als eine untergeordnete Barriere eingestuft (Bourgeois et al. 2014).

Allgemeinmediziner favorisieren nach initialer Beratung eine medikamentöse Therapie zur Behandlung von Insomnien, hingegen Angstzustände eher psychologisch behandelt werden (Siriwardena et al. 2010). Als Alternativen werden häufig pflanzliche Mittel, Antihistaminika, Neuroleptika oder Antidepressiva, wie z. B. niedrig dosiertes Amitriptylin angeboten, da diese als nebenwirkungsärmer, besser zur Langzeittherapie geeignet sowie möglicherweise im Vergleich mit den nicht-medikamentösen Therapien als mit einem Placeboeffekt behaftet eingestuft werden - und das trotz teilweise fehlender Zulassung zur Insomnie-Behandlung, wie im Fall von Amitriptylin (Everitt et al. 2014). Jedoch schätzten in einer Studie von 1980 Arbeitsmediziner Benzodiazepine im Vergleich zu Neuroleptika als Medikamente mit einer hohen angstlösenden Wirkung und einem insgesamt niedrigen Nebenwirkungsprofil ein, und das, obwohl den Benzodiazepinen ein hohes Abhängigkeitspotential zu geschrieben wurde. Nur für Patienten, die Maschinen bedienen und Auto fahren mussten, favorisierten die Befragten Neuroleptika (Sällström und Maasing 1980).

\subsection{6 Ärztliche Wahrnehmung der Risiken und Nebenwirkungen von BenzoZ}

Das Benzodiazepine und Z-Substanzen (BenzoZ) als Medikamente ohne Nebenwirkungen - abgesehen vom Abhängigkeitspotential - wahrgenommen werden, spiegelt auch die Befragung von in Pflegeheimen tätigen Ärzten und Pflegepersonal wider. Die alleinige Abhängigkeit führte allerdings nicht zu einem Absetzen des Medikaments, sondern begünstigte die Fortsetzung, zumal in 62\% der Fälle die Benzodiazepineinnahme vor dem Einzug ins Pflegeheim begonnen und das Absetzen mit einem pflegerischen Mehraufwand assoziiert wurde. Des Weiteren gaben über 80\% der Ärzte und Pflegekräfte an, dass die Medikamente auch bei Langzeitgebrauch ihre gewünschte Wirkung hätten und stuften daher das Absetzen - insbesondere auf Grund des hohen Alters der Bewohner - als „schwierig und unnötig“ ein (Bourgeois et al. 2014). In einer anderen Studie äußerten die befragten Ärzte, dass „Abhängigkeit im Alter nicht mehr so eine Rolle spielt“ (Flick et al. 2012) oder dass Benzodiazepine im Alter „nicht mehr so abhängig“ machen (Dybwad et al. 1997). Jedoch werden oft bei der Dosierung die veränderte Pharmakodynamik und -kinetik nicht bedacht (Zisselman et al. 1997). Ebenso befürchten die befragten Ärzte im Zusammenhang mit einem Entzug die Patienten zu verärgern (34\%), das Wiederauftreten der Schlafprobleme 
(24\%) oder eine Depression zu enthüllen (21\%). Letzteres wurde von einigen aber auch als Vorteil des Absetzens angesehen. Vier Prozent der Ärzte waren besorgt, wie Patienten den Ratschlag aufnehmen würden ein ,schädliches` Medikament abzusetzen, was sie selbst verordnet hatten (Iliffe et al. 2004).

Die Wahrnehmung des Risikos einer Abhängigkeit und des Missbrauchs scheint in den letzten Jahrzenten jedoch mehr in der das Bewusstsein der Ärzte gerückt zu sein. Anfang der 80er Jahre schätzen viele Ärzte, dass missbräuchliches Verhalten nicht die eigenen Patienten betraf (Chambers et al. 1983). Und in einer Studie von 1996 waren 65\% der 68 befragten Ärzte der Meinung, dass Benzodiazepine in therapeutischen Dosen keine Abhängigkeit auslösen; 35\% gaben an, dass dies selbst bei hohen Dosen unwahrscheinlich sei. Und 78\% hielten diesbezüglich eine Verwendung von bis zu vier Wochen für problemlos (Boixet et al. 1996). Möglicherweise bedingt durch neue Evidenz und angepasste Leitlinien nehmen viele Ärzte einen Wandel bezüglich Benzodiazepin-Verschreibungen wahr - jedoch auf unterschiedliche Art und Weise. Während sich einige Ärzte für den (nach heutigen Erkenntnissen) fehlerhaften Umgang mit diesen Substanzen in der Vergangenheit verantwortlich fühlten, empfanden andere die heutige eher negative Sichtweise als überbewertet (Sirdifield et al. 2013). In einer anderen Studie aus dem Jahr 1996 zeigten sich viele der Teilnehmer schon zurückhaltend bezüglich Benzodiazepin-Verordnungen und waren daran interessiert, ihren Patienten bei einem Entzug zu helfen (Holden et al. 1996). Dies mag zum einen die gesellschaftlich erwünschte Antwort sein, zum anderen nahmen 51\% der eingeladenen Ärzte nicht an der Studie teil. In einer Arbeit von 2014 war den befragten Ärzten das Problem der Toleranz- und Abhängigkeitsentwicklung bei Langzeitgebrauch von Benzodiazepinen und Z-Substanzen wohl bewusst - einige gaben explizit an, Verschreibungen aus diesem Grund zu vermeiden oder nur ein Rezept für ein bis zwei Wochen auszustellen (Everitt et al. 2014). Allerdings unterschätzen Studienteilnehmer die Menge der verordneten Benzodiazepine. Die von Boixet et al. befragten Ärzte gaben an, pro Woche und Arzt 14 Rezepte auszustellen; die tatsächlichen Anzahl an Rezepten betrug mehr als das Doppelte (Boixet et al. 1996).

Zusammenfassend lässt sich sagen, dass die Ergebnisse der Literaturrecherche bezüglich der Einschätzung von Hypnotika-Wirkung und Risiken insgesamt sehr unterschiedlich ausfallen; von effektiv bis uneffektiv bzw. von sicher bis unsicher. Jedoch konnten bereits mehrere Studien zeigen, dass Ärzte seit der Einführung der Z-Substanzen diese im Vergleich zu Benzodiazepinen als wirksamer, nebenwirkungsärmer - auch in Bezug auf Toleranz und Abhängigkeit - und als für ältere Menschen und zum Langezeitgebrauch besser geeignet wahrgenommen werden (Hoffmann 2013a; Siriwardena et al. 2006), obwohl sich diese Annahmen nicht wissenschaftlich belegen lassen (Siriwardena et al. 2008; Weaver 2015; Ströhle et al. 1999). Im interdisziplinären Vergleich scheinen Apotheker diesbezüglich kritischer zu sein (Hoffmann 2013a). Dass andere unerwünschte Nebenwirkungen, wie das erhöhtes Sturzrisiko oder kognitive Defizite, durch einen Dauerkonsum aufrechterhalten werden, wird in der Literatur nicht explizit diskutiert. Vielmehr wird die Abhängigkeit 
als das primäre Problem wahrgenommen, dass dann andere Probleme - vor allem ein resultierender Missbrauch, aber nachgeordnet auch Stürze und Gedächtniseinschränkungen mit sich bringt.

\subsection{7 Übertragung der Verantwortung}

Wird das problematische Einnahmeverhalten eines Patienten offensichtlich, wird die Schuld oft Psychiatern zugeschoben, die die Benzodiazepine initial verordnet hätten (Sirdifield et al. 2013; Rogers et al. 2007). So hatten in einer Studie von 1998 immerhin 17\% der Patienten, die ein psychiatrisches Krankenhaus verließen, ein neu verordnetes Benzodiazepin (Summers und Brown 1998) und 2007 beklagten britische Psychiater, dass die aktuellen Guidelines zum Benzodiazepin-Gebrauch zu restriktiv seien und um mehrere Indikationen ergänzt werden sollten, da ein erheblicher Teil der Verschreibungen off label erfolgt (Haw und Stubbs 2007). Dies steht im Widerspruch zu einer Studie von 1991, bei der mit Hilfe eines Fragebogens die Einstellung von 64 Allgemeinmedizinern und 42 Psychiatern erfasst wurde. Die Psychiater zeigten im Vergleich eine eher ablehnende Haltung gegenüber Benzodiazepinen und ein strikteres Verschreibungsverhalten (Kristensen und Fjermestad 1991). Auf der anderen Seite berichteten Psychiater im interprofessionellen Dialog über ihre Frustration, weil Hausärzte gemeinsamen Patienten Benzodiazepin-Rezepte verweigern würden (Johnson et al. 2007). Des Weiteren sehen Allgemeinmediziner die Schuld ebenfalls oft bei anderen bzw. früheren Hausärzten ihrer Patienten sowie Pflegeheimen (Siriwardena et al. 2010; Subelj et al. 2010; Dybwad et al. 1997). Gelegentlich wird die Verantwortung auch dem Patienten zugeschoben, der aufgeklärt wurde - dementsprechend die Risiken kennt - und sich nun selbst dazu entschließt, das Medikament fortzusetzen (Flick et al. 2012).

Auch bezüglich der Frage, wer für die Aufklärung und Beratung des Patienten zuständig ist, herrscht kein interdisziplinärer Konsens. Apotheker äußerten im Interview, dass Allgemeinmediziner hierauf größeren Wert legen sollten, insbesondere mit Blick auf schlafhygienische Maßnahmen, da diese im Vorfeld mehr Erfolg hätten als zu dem Zeitpunkt, zu dem der Patient in der Apotheke sein Medikament abholt. Allgemeinmediziner sahen Apotheker in der idealen Position, um mit dem Patienten über Nebenwirkungen und alternative Maßnahmen zu sprechen, zumal Apotheker Zugang zu Informationen über den Medikamentengebrauch des Patienten hätten, der Ärzten vorenthalten ist (Cheung et al. 2014). Jedoch steht in Deutschland weder Ärzten noch Apothekern ein Nachverfolgungssystem für Opitat- und Hypnotikaverschreibungen zur Verfügung, anders als beispielweise in den USA. Und ob Patienten, die mehrere Ärzte aufsuchen, um ein BenzoZ-Rezept zu erhalten, dieses immer in der gleichen Apotheke einlösen, sollte kritisch bedacht werden. Neben der Frage der Verantwortlichkeit konnten Wolde et al. zeigen, dass Allgemeinmediziner und Apotheke unterschiedlich motiviert werden, Patienten zu beraten (Wolde et al. 2008). Ärzte klärten ihre Patienten vermehrt auf, wenn sie von den Nachteilen des Benzodiazepingebrauchs überzeugt waren, wenn sie weniger negative Auswirkungen der Beratung sahen, 
wie z. B. eine Belastung der Arzt-Patient-Beziehung durch die Beratung, und sie sich hinsichtlich der Beratung kompetent fühlten. Apotheker berieten ihre Patienten vorwiegend dann, wenn sie mögliche positive Auswirkungen ihrer Beratung sahen. Beiden Berufsgruppen gemeinsam war, dass das Empfinden gesellschaftlichen Drucks ein Motivator war.

\subsection{Zunahme von Privatrezepten}

In den letzten zwei Jahrzehnten wurden insgesamt 75-80\% weniger Hypnotika im ambulanten Bereich zu Lasten der gesetzlichen Krankenversicherung (GKV) verschrieben (Schulz 2014; Lohse und Müller-Oerlinghausen 2016), wobei prozentual immer weniger Benzodiazepine und mehr Z-Substanzen verordnet werden. Mehrere Studien konnten jedoch zeigen, dass der Anteil auf Privatrezept verordneter Schlafmittel für Kassenpatienten zugenommen hat (Diemert et al. 2013; Hoffmann et al. 2009; Hoffmann et al. 2010; Hoffmann et al. 2006). Mittlerweile ist fast jedes zweite Hypnotikumrezept ein Privatrezept (Diemert et al. 2013).

Es scheinen aber wirkstoffspezifische Unterschiede zu existieren: 2011 lag der Anteil an Privatverordnungen für Flunitrazepam bei $68 \%$. Auf Grund des bekannten hohen Missbrauchspotentials fällt das Medikament mittlerweile, wie erwähnt, unter die Betäubungsmittelverordnung. Dies führte zu einem Gebrauchsrückgang, obwohl auch Betäubungsmittel privat verschrieben werden können - nur bedarf es einer ausführlicheren Dokumentation (Hoffmann und Glaeske 2014). Zudem konnten Hoffmann et al. zeigen, dass Zolpidem häufiger privat verordnet wird als Zopiclon. Zolpidem besitzt eine kürzere Halbwertzeit und möglicherweise ein höheres Missbrauchs- und Abhängigkeitsrisiko. An anderer Stelle wird der Unterschied zu Zopiclon eher positiv bewertet (Dündar et al. 2004). So würde Zolpidem zu einem schnelleren Einschlafen führen und weniger Rebound-Schlafstörungen verursachen. Allerdings gibt es nur wenige Studien, die die unterschiedlich Z-Substanzen miteinander vergleichen (Dündar et al. 2004); hier bestehen Forschungslücken (Hoffmann et al. 2010).

In einer anderen Arbeit heißt es, dass vorwiegend größere Packungen und höhere Hypnotika-Dosen so verschrieben werden (Hoffmann et al. 2006). Überregional verlässliche Daten zum Thema Privatrezepte für kassenärztlich Versicherte sind jedoch schwer erhältlich, da diese Rezepte weder durch die GKV noch durch die privaten Versicherungen erfasst werden. Eventuell können hierzu in Zukunft Routinedaten - also Daten, die im ambulanten Alltag durch die Nutzung eines Arztpraxen-Informationssystems (AIS) generiert werden - genutzt werden. Jedoch gibt es noch keine wissenschaftliche Datentransferschnittstelle für alle deutschen AIS (Weiß und Hauswaldt 2015).

Noch weniger als über die Menge der Hypnotika, ist über die Beweggründe der ausstellenden Ärzte bekannt, GKV-Versicherten ein Privatrezept zu geben. 


\subsection{Motive für das Ausstellen eines Privatrezeptes}

Hoffmann untersuchte 2014 mit Hilfe eines schriftlich zugesandten Fragebogens die Einstellung von zufällig ausgewählten Hausärzten und Apothekern in Bezug auf HypnotikaRezepte und fand heraus, dass laut Einschätzung beider Berufsgruppen Patienten mit Langzeitgebrauch öfter Privatverordnungen erhalten (Hoffmann et al. 2014). Bedingt sei dies durch die Vorgaben der Arzneimittel-Richtlinie (AMRL) oder weil der Patient ein Rezept wünscht (Wunschverordnung). Die Entlastung des Praxisbudgets spielte eine untergeordnete Rolle. Im Gegensatz zu den Ärzten schätzen insgesamt 40 \% der befragten Apotheker das Nichterscheinen in den Kassendaten als einen wichtigen oder sehr wichtigen Punkt für das Ausstellen eines Privatrezeptes ein; für 40\% der befragten Ärzte hat dies keine Relevanz. An anderer Stelle wurde gemutmaßt, dass Ärzte die Arzt-PatientenBeziehung gefährdet sehen, wenn sie das Medikament nicht verordnen würden und daher den Weg über das Privatrezept wählten (Hoffmann et al. 2006) - wobei dies nicht erklärt, warum die Verschreibung nicht über ein GKV-Rezept erfolgt.

Ob sich der durch niedergelassene Psychiater ausgestellte Anteil an Privatrezepten in den letzten Jahren ebenfalls erhöht hat und ob sich deren Beweggründe von denen der Allgemeinmediziner unterscheiden, ist ebenfalls nicht bekannt. 


\subsection{Zusammenfassung des Forschungsstandes}

Im Zusammenhang mit Benzodiazepinen und Z-Substanzen gibt es eine Reihe von Studien, die sich den soziodemographischen Eigenschaften des stereotypischen Patienten, aber auch des stereotypischen Verschreibers widmen (s. 2.5.1, 2.5.2 und 2.5.3). Ebenfalls ist bekannt, dass in den letzten 20 Jahren zwar immer weniger Schlaf- und Beruhigungsmittel zu Lasten der gesetzlichen Krankenkassen verschrieben wurden (Lohse und MüllerOerlinghausen 2016); dass aber ein großer Anteil über ein Privatrezept verordnet wird (Hoffmann et al. 2014; Schulz 2014) und der Rückgang daher geringer sein dürfte als die aktuellen Zahlen belegen. Sucht man in der Literatur konkret nach ärztlichen Meinungen zum Thema Hypnotika, erhält man Antworten auf die Frage, wann Ärzte ein solches Medikament verwenden (s. 2.5.4), welche Risiken sie sehen - hier dominiert das Problem der Abhängigkeitsentwicklung (s. 2.5.6) - und welche alternativen Behandlungsoptionen sie nutzen und wie sie deren Erfolg im Vergleich zu BenzoZ einschätzen (s. 2.5.5). Warum in Deutschland nicht alle GKV-Patienten bei gegebener Indikation ein Kassenrezept für diese Medikamente erhalten oder eben kein Rezept bei nicht gegebener Indikation, sondern oft ein Privatrezept ausgestellt bekommen, ist nicht bekannt. Hierzu gibt es einige Mutmaßungen sowie eine Fragebogenstudie, die sich explizit hiermit befasst (Hoffmann et al. 2014). Jedoch waren bezüglich der Frage nach dem Warum nur vier Antwortmöglichkeiten vorgegeben (Vorgaben der Arzneimittelrichtlinie, Wunschverordnung, Nichterscheinen in den Kassendaten, kritische Einstellung zur Verordnung). Ob weitere Gründe die Wahl der Rezeptform beeinflusst haben, vermag die Arbeit von Hofmann et al. nicht zu beantworten. Eine Interviewstudie mit in Deutschland niedergelassenen Allgemeinmedizinern zu diesem Phänomen gibt es nach Wissen der Autorin bis heute nicht. 


\section{$3 \quad$ Fragestellung und Ziel der Arbeit}

Der Sinn dieser qualitativen Studie ist es, die Motive der niedergelassenen Allgemeinmediziner bezüglich Hypnotika-Privatverordnungen für Kassenpatienten besser zu verstehen. Wie im vorherigen Absatz dargestellt, besteht hier eine Forschungslücke. Es ist nicht bekannt, warum Ärzte diese Form der Verschreibung für Benzodiazepine und Z-Substanzen verwenden. Will man aber die tatsächlich verordneten Mengen an Schlaf- und Beruhigungsmitteln in Deutschland nicht nur abschätzen (Diemert et al. 2013), muss zunächst geklärt werden, warum zur Zeit viele Rezepte für Kassenpatienten als Privatverordnung ausgestellt werden und somit in keiner Statistik auftauchen. Hierzu sollen Allgemeinmediziner interviewt werden.

Die Bildung einer neuen Theorie zur Erklärung dieses Phänomens mit Hilfe der Grounded Theory ist primäres Ziel dieser Arbeit.

Des Weiteren sollen einige Punkte - wenn möglich - konkret herausgearbeitet werden:

Haben wahrgenommene Patiententypen aus Sicht der Ärzte einen Einfluss? Und wenn ja, gibt es ähnlich dem Patiententyp, der ein Schlafmittelrezept „,verdient“ hat (Sirdifield et al. 2013; Rogers et al. 2007), eine Gruppe von Kassenpatienten, die bevorzugt ein Privatrezept erhalten? Oder gibt es umgekehrt einen Patiententypus, der ein Kassenrezept oder gar kein BenzoZ-Rezept erhält?

Sehen Allgemeinmediziner ihr Verschreibungsverhalten von Benzodiazepinen und ZSubstanzen durch verschiedene Diagnosen unterschiedlich beeinflusst? Wenn ja, wie äußert sich dies aus Sicht der Ärzte?

Ist die Privatverordnung von den Teilnehmern als dauerhafte Lösung gedacht oder stellt sie eine Übergangslösung dar? Soll z. B. die Bereitschaft des Patienten zur Entwöhnungstherapie durch den finanziellen Aufwand verstärkt werden?

Welchen Einfluss haben - aus Sicht der Allgemeinärzte - Patienten selbst, aber auch Mitbetreuer, wie Psychologen oder Psychiater auf das Verordnungsverhalten? Spielen die gesetzlichen Krankenkassen in dem Entscheidungsprozess für oder gegen ein Privatrezept eine Rolle?

Wie empfinden die Befragten die Vorgaben der aktuellen Arzneimittelrichtlinie, soweit diese bekannt sind? 


\section{$4 \quad$ Material und Methoden}

\subsection{Studiendesign}

Da es im Rahmen dieser Arbeit nicht um quantitativ-statistische Angaben, wie Verordnungshäufigkeit und -dauer, den prozentualen Anteil einer Rezeptform oder auch das Abprüfen von Kenntnissen geht, sondern darum, zu verstehen, warum dieses Verhalten Kassenpatienten ein BenzoZ-Privatrezept auszustellen - auftritt, bietet sich ein qualitatives Studiendesign an. Möglicherweise sind die Motive von Ärzten für das Ausstellen von Privatrezepten wesentlich individueller und komplexer, als dass sie mit einem Fragebogen erfasst werden können. Eine Interviewstudie zu diesem Thema existierte bisher nicht. Im Rahmen dieser Arbeit sollten daher niedergelassene Allgemeinmediziner teilstrukturiert, also leitfadenstützt, interviewt werden. Diese Vorgehensweise erschien zielführend, da durch vorformulierte Stichpunkte sichergestellt werden konnte, das relevante Aspekte in jedem Interview zur Sprache kamen; andererseits hatten die Befragten genug Freiraum, von ihren Meinungen und Erfahrungen zu erzählen. Die Planung der Studie (Stichprobengröße, Sampling, Studienpopulation und -gebiet) erfolgte durch Katharina Schmalstieg.

\subsubsection{Stichprobengröße und Sampling}

Geplant war das Erreichen einer theoretischen Sättigung. Laut Strauss und Corbin bedeutet dies die Forstsetzung der Studie, bis sich kein neuer Erkenntnisgewinn mehr ergibt, ,alle paradigmatischen Elemente (...) berücksichtigt" und „die Beziehungen zwischen den Kategorien (...) validiert“ sind (s. 4.4.2 ff.) (Strauss und Corbin 2010). Initial wurde davon ausgegangen, dass hierzu 15-25 Interviews nötig wären, da thematisch verwandte Studien, die mit teilstrukturierten Interviews arbeiteten, eine Sättigung nach 22 (Rogers et al. 2007) bzw. 23 (Cook et al. 2007) Interviews erreichten. Anfänglich erfolgte eine Cluster Sampling-ähnliche Rekrutierungsstrategie, indem alle Ärzte im Studiengebiet, die die Einschlusskriterien erfühlten (s. 4.2) zu einer Teilnahme eingeladen wurden. Im Laufe der Studie und nach Beginn der Datenauswertung im Sinne der Grounded Theory (s. 4.4.2 ff.) erfolgte ein theoretisches Sampling - also die gezielte Auswahl der Studienteilnehmer auf Grund der bis dato als relevant identifizierten Konzepte (Strauss und Corbin 2010).

\subsection{Studienpopulation und -gebiet}

Da die Gruppe der Hausärzte die meisten Schlaf- und Beruhigungsmittel verordnet (Schaufler und Telschow 2015), sollten zunächst alle niedergelassenen Allgemeinmediziner und hausärztlich tätigen Internisten (Fachärzte und Weiterbildungsassistenten) in einem kleinen Rekrutierungsgebiet zur Studienteilnahme eingeladen werden. Die Größe des Ge- 
bietes ergab sich aus der Annahme, dass circa 20 Interviews nötig wären, um eine thematische Sättigung zu erreichen (s. 4.1.1). Hierzu wurde primär die Region des Eichsfelds gewählt (südöstliches Niedersachsen und nordwestliches Thüringen), da einige in Göttingen und Umgebung niedergelassene Ärzte bereits innerhalb des Projektes „...da gab es wunderbare Schlaftabletten - Verordnungen von Benzodiazepinen und Z-Substanzen an der Schnittstelle von Krankenhaus und Hausarzt“ interviewt wurden und erneute Befragungen vermieden werden sollten. Des Weiteren erfolgten nach Beginn der Auswertung und bis zum Erreichen der theoretischen Sättigung ergänzend Interviews im großstädtischen Bereich Hannover (theoretisches Sampling). Alter, Geschlecht und die Dauer der Niederlassung sowie die Art der Praxis (Praxisgemeinschaft oder Einzelpraxis) und die Qualifikation (Weiterbildungsassistent oder Facharzt) spielten bei der Rekrutierung der Teilnehmer keine Rolle. Von der Studie ausgeschlossen wurden Ärzte, die ihre Praxis aufgegeben hatten und sich zum Zeitpunkt des Interviews länger als drei Monate im Ruhestand befanden.

\subsection{Ablauf der Studie}

\subsubsection{Entwicklung des Interview-Leitfadens}

Zunächst wurde von Katharina Schmalstieg der Leitfaden anhand von bereits in der Abteilung verwendeter Leitfäden erstellt, insbesondere nach dem Vorbild der Ärzteinterviews innerhalb des oben genannten Projektes „... da gab es wunderbare Schlaftabletten“. Dieses wurde im Gegensatz zu der vorliegenden Arbeit vom Bundesgesundheitsministerium (BMG) gefördert und befasste sich mit der Verordnung von Benzodiazepinen und Z-Substanzen an der Schnittstelle zwischen ambulanter und stationärer Versorgung. Der Leitfaden wurde entsprechend der vertiefenden Thematik dieser eigenständigen Arbeit geändert (s. Anhang 8.5) und entstand im wechselseitigen Austausch mehrerer Mitarbeiter des Instituts. Es erfolgten zwei Probeinterviews; anschließend wurde der Leitfaden nochmals überarbeitet und um die Themen BenzoZ im Notdienst, Vorgaben bzw. Hinweise des AIS bei BenzoZ-Verschreibungen und persönlicher Gebrauch von Schlaf- und Beruhigungsmitteln ergänzt.

\subsubsection{Rekrutierung der teilnehmenden Praxen und Ärzte}

Ziel war - zumindest für das niedersächsische Eichsfeld - die Teilnahme vieler, wenn möglich aller Hausärzte der Region. Die Ermittlung der Adressen und Telefonnummer erfolgte durch Katharina Schmalstieg durch einen Abgleich von bereits im Institut vorhandenen Ärztelisten der Region mit den Gelben Seiten (Gelbe Seiten 2018).

Bei einer angenommenen Rücklaufquote von 30-50\% war zunächst geplant 50 Praxen eine entsprechende Einladung, das Informationsblatt zur Studie sowie einen Antwortbogen per Post oder Fax zukommen zu lassen. Jedoch konnten Studien bereits zeigen, dass Zeitman- 
gel und bürokratischer Aufwand wesentliche Hemmfaktoren für eine Studienteilnahme sind (Rosemann und Szecsenyi 2004; Hummers-Pradier et al. 2008). Daher wurden einige Ärzte von Katharina Schmalstieg persönlich mittels Telefon kontaktiert (Arzt zu Arzt) und erhielten anschließend die entsprechenden Unterlagen. Dieser Weg der Rekrutierung erwies sich als zielführend und wurde somit beibehalten. Es wurden alle Ärzte im primären Rekrutierungsgebiet angerufen. Da nicht alle mitmachen wollten, wurden die Außengrenzen des Gebiets nach und nach erweitert, also auch Praxen in Thüringen kontaktiert. Jeder, der Interesse hatte, wurde auch interviewt. Auf die gleiche Weise wurden die Teilnehmer aus dem Raum Hannover rekrutiert. Die Studie wurde mit dem Forschungstitel „Hypnotika in der Praxis“ angekündigt. Bei Interesse erfuhren alle kontaktierten Personen, dass es sich hierbei um ein Promotionsprojekt handelt. Wie aus dem Informationstext zur Studie ersichtlich, erhielt jeder Arzt eine Aufwandsentschädigung von 50 Euro (s. 8.2).

\subsubsection{Durchführung der Interviews}

Alle Interviews dieser Studie - inklusive der beiden Probeinterviews - wurden, nach vorheriger zweitägiger Schulung durch Dr. Susanne Friese (QUARC 2018), von Katharina Schmalstieg durchgeführt. Wie bereits aus 4.3.2 ersichtlich, ist Katharina Schmalstieg Ärztin und war zum Zeitpunkt der Datenerhebung und -auswertung als wissenschaftliche Mitarbeiterin am Institut für Allgemeinmedizin in Göttingen angestellt. Zeit und Ort der Interviews (Praxis oder Privatwohnung, in einem Fall auch das Institut) wurden von dem jeweiligen Befragten bestimmt. Die Interviews erfolgten jeweils einmalig. Zunächst wurden alle Studienteilnehmer über die Studie aufgeklärt und unterschrieben die Einverständniserklärung (s. 8.3). Außer der Interviewenden und dem jeweiligen Befragten war keine weitere Person anwesend. Von allen Interviews wurde eine Audioaufnahme angefertigt; zusätzlich wurde unmittelbar nach jedem Gespräch von der Interviewenden - aber in Abwesenheit des Interviewpartners - eine Forschungsnotiz (Memo) diktiert. Hierbei wurden noch einmal die Rahmenbedingungen des Interviews sowie eventuelle Störungen von außen festgehalten. Nonverbales, mögliche Probleme, die Wahrnehmung des Interviewpartners sowie die Selbstwahrnehmung wurden reflektiert.

\subsubsection{Datenerhebung im Qualitätszirkel}

Entsprechend dem Prinzip des theoretischen Samplings (s. 4.1.1) wurde bereits vor Abschluss der Datenerhebung mit der Auswertung der Interviews begonnen. Hierbei zeigte sich der Wunsch einiger Studienteilnehmer, die Verordnung von Schlaf- und Beruhigungsmitteln auf Privatrezept mit Kollegen im Rahmen eines Qualitätszirkels (QZ) zu diskutieren. Nach vorheriger Absprache und auf Wunsch der Gruppe hielt Katharina Schmalstieg zunächst einen Vortrag über die nach aktuellen Leitlinien und AMRL korrekte Verordnung von Schlafmitteln, an den sich eine Gruppendiskussion anschloss. Hierbei wurde die Frage, ob man durch Privatrezepte den Hypnotika-Konsum eines Patienten einschränken kann, offen besprochen (Vergleiche Ergebnis- und Diskussionsteil). Es handelte es 
sich dabei um einen freien kollegialen Austausch; die Diskussion orientierte sich nur grob an dem für die Einzelinterviews verwendeten Leitfaden, und es wurden keine demographischen Daten erhoben. Das Gespräch wurde - wie die Einzelinterviews - auf Tonband aufgezeichnet. Zuvor wurden alle Teilnehmer über die Verwendung der Daten aufgeklärt und gaben ebenfalls ihr schriftliches Einverständnis (s. 8.4). Da Katharina Schmalstieg nicht nur Beobachtende war, sondern zeitgleich in der Rolle der Referentin fungierte, erhielten die QZ-Teilnehmer keine Aufwandsentschädigung.

\subsubsection{Datenrecherche zu KV-, Krankenkassen-, Apotheken- und Apothekenkammervorgaben}

Ebenfalls wurde während der Auswertung deutlich, dass die interviewten Ärzte die Vorgaben oder Richtlinien der Kassenärztlichen Vereinigung (KV) und der Krankenkassen bezüglich Hypnotikaverordnungen sehr unterschiedlich interpretierten und dass dies Auswirkungen auf das jeweilige Verschreibungsverhalten hatte. Um die Vorgaben zu überprüfen, fand jeweils ein Telefonat zwischen Katharina Schmalstieg und der Arzneimittelberatung der KV Niedersachsen und der KV Bayern beziehungsweise der Beratungsapotheke der Allgemeinen Ortskrankenkasse (AOK) und der Telefonauskunft der BARMER statt. Des Weiteren wurde die Auskunft einer Göttinger Apotheke und der Apothekenkammer Niedersachsen eingeholt. Nach jedem Gespräch wurde unmittelbar ein Gedächtnisprotokoll angefertigt, das in die Datenauswertung einbezogen wurde.

\subsubsection{Zeitpunkt der Datenerhebung}

Es wurde jeweils ein Probeinterview im Dezember 2015 und im Januar 2016 geführt. Nach der Überarbeitung des Leitfadens erfolgten die anderen Interviews zwischen April 2016 und Januar 2017. Die Datenerhebung im Qualitätszirkel erfolgte im November 2016. Die Recherche der KV- und Krankenkassenangaben geschah im November 2016 sowie im September 2017. Die Telefonate mit Apotheke und Apothekenkammer fanden im Dezember 2017.

\subsection{Datenauswertung}

\subsubsection{Transkription der Interviews}

Alle Audiodateien wurden von Mitarbeitern des Instituts wortgenau unter Berücksichtigung der Transkriptionsregeln (s. Anhang 8.6) verschriftlicht (= transkribiert). Die Transkripte wurden von Katharina Schmalstieg durch nochmaliges Abgleichen mit der Audiodatei auf ihre Richtigkeit überprüft und pseudonymisiert. Anschließend wurde das entsprechende Transkript jedem Einzelinterviewteilnehmer mit der Möglichkeit zugesandt, Aussagen zu streichen oder zu ändern. Da diese Korrekturoption von keinem der Einzelinterviewteilnehmer genutzt wurde, wurde hierauf im Qualitätszirkel verzichtet. Zur Auswer- 
tung wurden ausschließlich die bereits pseudonymisierten Daten verwendet. Die Memos wurden ebenfalls getippt und hierzu herangezogen. Die pseudonymisierte Abschrift der QZ-Diskussion wurde separat ausgewertet. Die erhobenen Daten (Transkripte und Memos) wurden mit Hilfe der Grounded Theory nach Strauss und Corbin bearbeitet. Dies geschah computergestützt unter Verwendung des Programms MAXQDA 12. Die Wahl der Methode erfolgte durch Katharina Schmalstieg nach Beratung mit mehreren Mitarbeitern des Instituts und geschah mit Hinblick auf die Fragestellung, wie im Folgenden erläutert wird.

\subsubsection{Grounded Theory}

Die Grounded Theory, die von einigen nicht nur als Methode, sondern als Forschungsstil beschrieben wird (Mey und Mruck 2009), wurde 1967 von Glaser und Strauss etabliert (Glaser und Strauss 1967), und später von Strauss und Corbin weiter verändert (Corbin und Strauss 1993). Sie erlaubt die „Entwicklung einer in den Daten begründeten Theorie menschlichen Handelns" (Wollny und Marx 2009). Eine neue Theorie soll gewonnen, nicht etwa eine bestehende Theorie an das Material herangetragen werden. Ziel ist nicht die Feststellung von Häufigkeiten oder das alleinige Beschreiben, sondern das Verstehen sozialer Phänomene (Strauss 1998) durch den Nachvollzug individuellen Handels (Mey und Mruck 2009). Bezogen auf die Fragestellung dieser Arbeit schien die Grounded Theory daher als geeignete Auswertungsmethode. Grundlegend sind dabei zum einen das theoretische Sampling (s. 4.1.1), zum anderen die Aufhebung der Trennung von Forscher und Forschungsgegenstand; so werden Datenerhebung- und Analyse durch denjenigen beeinflusst, der die Interviews führt und auswertet. Dies soll fortwährend und bewusst reflektiert werden. Die Datenanalyse wird als Kodieren bezeichnet und kann in drei Schritt unterteilt werden: offenes, axiales und selektives Kodieren. Die einzelnen Schritte sowie die einzelnen Forschungsphasen finden jedoch zeitgleich und wechselseitig statt; so begann die Auswertung bereits nach dem ersten Interview und erfolgte iterativ - also als ein sich wiederholender Vergleich der bisherigen Ergebnisse mit neu gewonnenen Daten (Wollny und Marx 2009). Wie bereits erwähnt, wurden Daten bis zum Erreichen der theoretischen Sättigung erhoben, was nach 17 Einzelinterviews und einer Gruppendiskussion der Fall war.

\subsubsection{Offenes Kodieren}

In diesem ersten Schritt wurden die Transkripte sehr genau gelesen und einzelne Konzepte - wie beispielsweise Äußerungen, Ideen, beschriebene Beobachtungen und Handlungen identifiziert. Ein Konzept konnte aus einem einzelnen Wort oder Halbsatz bestehen, oder sich über mehrere Zeilen erstrecken. Anschließend wurde jedes Konzept mit einem Kode benannt. Ein Kode-Name konnte sowohl neu erfunden als auch allgemein bekannt sein und unter Rückgriff auf Vorwissen gewählt werden („Geborgter Kode“). Kodes, die sich an der Wortwahl des Textes orientieren, wurden als In-vivo-Kodes bezeichnet (Mey und Mruck 2009). Ziel dieses Schritts war das Aufdecken möglichst vieler eventuell relevanter 
Konzepte. Zu diesem Zweck war es wichtig, offen und ohne Vorannahmen an den Text heranzugehen (Prinzip der Offenheit) (Strauss und Corbin 2010). Um keine Inhalte zu übersehen, wurden die Transkripte zu Beginn der Auswertung in einem interdisziplinären Team mit methodisch erfahrenen Mitarbeitern des Instituts für Allgemeinmedizin kodiert.

\subsubsection{Axiales Kodieren}

Als nächstes wurden Konzepte höherer Ordnung identifiziert (Kategorien), indem die bereits bekannten Konzepte des offenen Kodierens zu Ober- oder Subkategorien gruppiert wurden. Jede Ober- und Subkategorie wurde ebenfalls mit einem Kode benannt. Jedoch orientierten sich die Namen im Vergleich zum ersten Schritt weniger am Text und besaßen ein höheres Abstraktionsniveau. Anschließend wurden die Kategorien zueinander in Beziehung gesetzt. Zur systematischen Darstellung dieser Beziehungen wurde das paradigmatische Modell verwandt (Strauss und Corbin 2010). Dies ist in Abbildung 2 dargestellt und schlägt die Ordnung der Kategorien unter den angegebenen Gesichtspunkten vor.

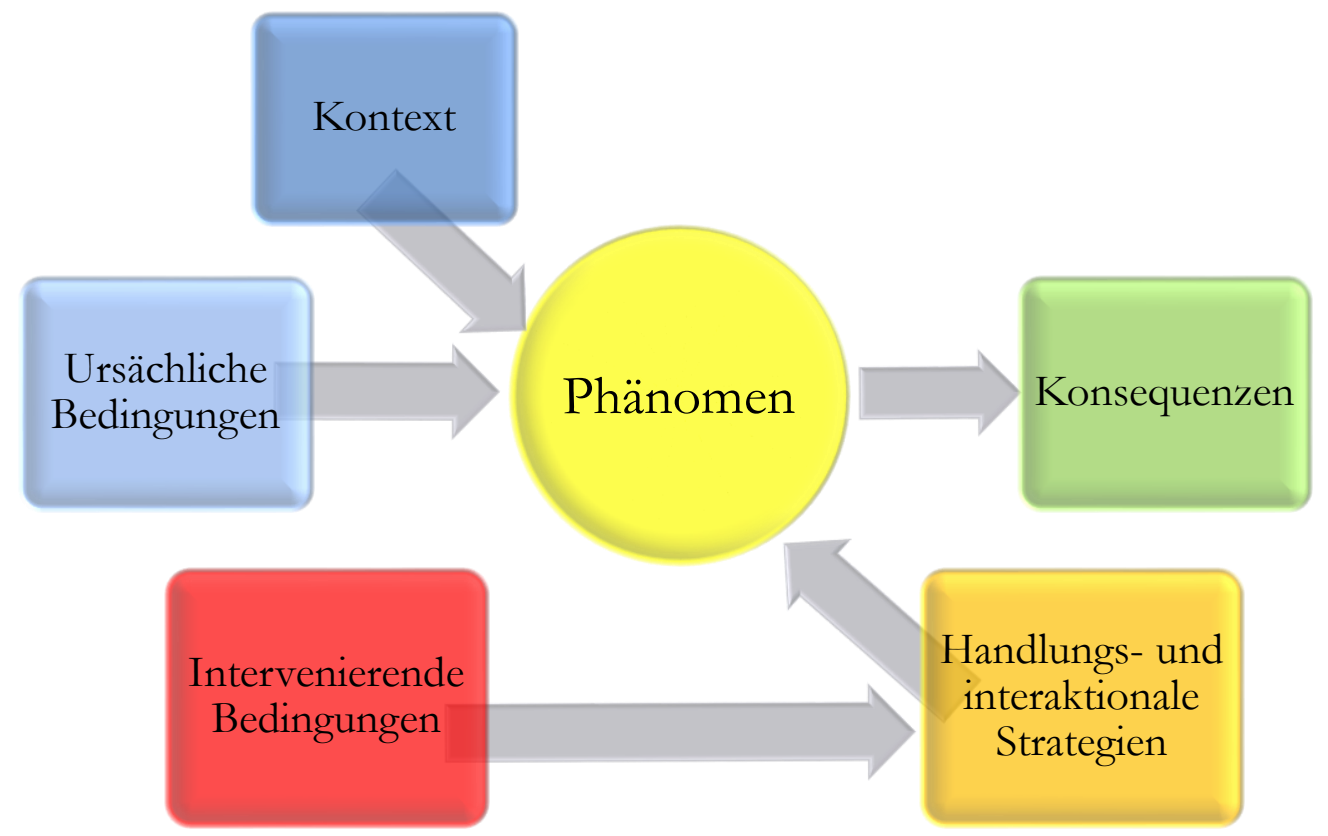

Abbildung 2: Das paradigmatische Modell

Das Phänomen kann hierbei auch als zentrale Idee verstanden werden. Die ursächlichen Bedingungen tragen zu seiner Entstehung und Aufrechterhaltung bei, während der Kontext die übergeordneten zeitlichen und örtlichen Einflüsse beschreibt (Mey und Mruck 2009) oder, nach der Formulierung von Strauss und Corbin, die Bedingungen, in denen „die Handlungs- und interaktionalen Strategien stattfinden“ (Strauss und Corbin 2010). Die Letzteren werden genutzt, um ein Phänomen „zu bewältigen, damit umzugehen, es auszuführen oder darauf zu reagieren“" (Strauss und Corbin 2010), während die intervenierenden Bedingungen Einfluss auf die Ausgestaltung des Phänomens haben, indem sie die verwen- 
deten Strategien erleichtern oder hemmen. Die Resultate einer Handlung oder Interaktion werden als Konsequenzen bezeichnet.

\subsubsection{Selektives Kodieren}

Das selektive Kodieren kann als abstrakte Fortsetzung des axialen Kodierens verstanden werden (Mey und Mruck 2009). Hierbei wurden die Kernkategorien herausgearbeitet und in Beziehung zu den anderen Kategorien gesetzt bzw. die schon erarbeiteten Beziehungen überprüft, so dass die entstandene Theorie als roter Faden der Ergebnisse sichtbar wurde. Durch weitere Datenerhebung wurden Lücken aufgefüllt und die neu entstandene Theorie validiert. Wichtig, und wie bereits erwähnt, ist der Hinweis von Strauss und Corbin, dass die drei Schritte des Kodierens nicht linear und voneinander getrennt erfolgen müssen (Strauss und Corbin 2010).

\subsubsection{Dimensionierung}

Die Ausprägung oder Bandbreite einer Ober- oder Subkategorie wird als Dimension bezeichnet. Diese kann sowohl qualitativ (zufrieden - unzufrieden) als auch quantitativ sein (viel - wenig). Alle der Kategorie zugehörigen Daten befinden sich somit auf einem Kontinuum, das von einem Extrem (z. B. sehr zufrieden) bis zum gegenteiligen Extrem (z. B. sehr unzufrieden) reichen kann.

\subsubsection{5 Überprüfung des Kodesystems}

Durch die Parallelität der Schritte konnten die letzten zwei Interviews beim Kodieren in das bereits ausgearbeitete Modell eingefügt und die Theorie somit überprüft werden. Dabei erfolgte das Kodieren aller Interviews durch Katharina Schmalstieg. Jedoch wurde zur Plausibilitätsprüfung das Kodesystem auswertungsbegleitend im Rahmen der abteilungsinternen Forschungswerkstatt mit Mitarbeitern des Instituts sowie mit Kristina Meier M.A. vom Methodenzentrum Sozialwissenschaften der Georg-August-Universität Göttingen diskutiert. Als Soziologin und wissenschaftliche Mitarbeiterin ist sie dort u. a. für die qualitative Methodenberatung zuständig.

\subsubsection{MAXQDA}

MAXQDA ist eine Software der VERBI GmbH (Berlin) und eine Weiterentwicklung des von Udo Kuckartz kreierten Programms winMAX (Flick 2016). Sie ermöglicht eine einfachere Analyse qualitativer Daten, wie beispielsweise transkribierter Interviews (MAXQDA 2018). Mit Hilfe der Funktion MAXMAPS können Kategorien und Subkategorien sowie deren Beziehung zueinander auch graphisch dargestellt werden. In dieser Arbeit wurde die Version MAXQDA 12 verwendet. Die Lizenz wurde über das Institut für Allgemeinmedizin/Universitätsmedizin Göttingen erworben. 


\subsection{Datenschutz und Genehmigung durch die Ethikkommission}

Die Genehmigung der Ethik-Kommission zu dieser Arbeit erfolgte als Amendment III im Rahmen des BMG-Forschungsprojektes „... da gab es wunderbare Schlaftabletten“ - Verordnungen von Benzodiazepinen und Z-Substanzen an der Schnittstelle von Krankenhaus und Hausarzt (Antragsnummer 25/2/14).

Alle Projektmitarbeiter wurden mit den Bestimmungen des Datenschutzes vertraut gemacht und auf die Wahrung des Datengeheimnisses nach \5 BDSG verpflichtet. Alle Studienteilnehmer (zu einem Interview bereite Ärzte) wurden ausführlich über die Studie aufgeklärt und mussten schriftlich ihr Einverständnis erklären. Hierbei wurden die Teilnehmer explizit auf die Freiwilligkeit der Teilnahme und den vertraulichen Umgang mit den erhaltenen Informationen hingewiesen. Die Teilnehmer konnten jederzeit und ohne Angabe von Gründen ihr Einverständnis zurückziehen und aus der Studie ausscheiden, ohne dass sich daraus Nachteile für sie ergeben hätten. Bei Widerruf der Zustimmung wären die entsprechenden Daten sofort und endgültig gelöscht worden. Die Teilnehmer konnten vor, während und nach der Datenerhebung (Interviews) Fragen im Gespräch oder per E-Mail und Telefon äußern, die entsprechend beantwortet wurden. Die Audiodaten der aufgenommenen Interviews wurden wortgenau transkribiert und anschließend in pseudonymisierter Form im Institut für Allgemeinmedizin gespeichert. Jegliche Nutzung dieser Daten erfolgt ebenfalls nur in pseudonymisierter Form. Am Ende der Studie wurden die Transkriptionen in einem verschließbaren Schrank archiviert und werden nach Ablauf von 10 Jahren unwiederbringlich vernichtet werden. Nicht pseudonymisierte Daten wurden nach Ablauf der Studie gelöscht. Im Fall des Widerrufs der Zustimmung durch die Teilnehmer würden die Daten (einschließlich der angefertigten Verschriftlichungen) sofort gelöscht werden.

\subsection{Vorstellung und Veröffentlichung der Daten}

Auf Grund der Tatsache, dass die interviewführende Person selbst Allgemeinmedizinerin ist, wurden Auszüge der Daten während der Auswertungsphase fachfremden Personen vorgestellt und mit diesen diskutiert. Dies diente der Umsetzung des Prinzips der Offenheit - und somit einer Auswertung ohne ggf. vorhandene berufsspezifische Vorannahmen. Auszüge wurden zum einen im September 2016 im Rahmen der 5. Göttinger Forschungswerkstatt der Klinik für Palliativmedizin der Universitätsmedizin Göttingen präsentiert und diskutiert; zum anderen im Doktorandenkolloquium des Instituts für Sportwissenschaften der Universität Göttingen, in dem Personen mit anderem beruflichen Hintergrund, aber Erfahrung mit ähnlichen Fragestellungen bzw. Methoden bereit waren, die Daten bzw. Ergebnisse kritisch zu diskutieren. Nach Abschluss der Auswertung wurden die Daten im Mai 2017 auf der 84. Konferenz des EGPRN (European General Practice Research Network) einem internationalen Fachpublikum vorgestellt. Die Publikation der Ergebnisse ist ausstehend. 


\section{$5 \quad$ Ergebnisse}

\subsection{Teilnehmer der Einzelinterviews}

Insgesamt wurden 43 Ärzte (23 Männer, 20 Frauen) in 29 Praxen erfolgreich kontaktiert und zur Teilnahme an der Studie eingeladen. Hiervon lehnten 24 Ärzte (11 Männer, 13 Frauen) ein Interview ab und gaben an, kein Interesse oder keine Zeit zu haben. Jedoch wurde nicht immer ein Grund für die Absage genannt. Zwei Ärzte hatten ihre Praxis zum Zeitpunkt der Datenerhebung bereits länger als 3 Monate aufgegeben. Siebzehn Ärzte erklärten sich zu einem Interview bereit (Teilnehmerquote 39\%); hiervon waren 41\% Frauen (7 von 17) und 59\% Männer (10 von 17). Namen werden aus datenschutztechnischen Gründen nicht genannt. Eine Übersicht der demografischen Daten ist Tabelle 2 zu entnehmen.

Tabelle 2: Demographische Daten der Studienteilnehmer

\begin{tabular}{|l|c|c|}
\hline & Frauen & Männer \\
\hline Anzahl der Teilnehmer & 7 & 10 \\
\hline Allgemeinmedizin & 4 & 7 \\
Innere Medizin & 2 & 1 \\
Praktischer Arzt & 1 & 2 \\
\hline Facharzt & 5 & 8 \\
Arzt in Weiterbildung & 1 & \\
Praktischer Arzt & 1 & 2 \\
\hline Mittlerer Zeitpunkt der Facharztqualifikation vor X Jahren & 10 & 14,4 \\
(Spannweite in Jahren) & $(5-18)$ & $(3-33)$ \\
\hline Mittlere Dauer der Niederlassung in Jahren & 11,9 & 17,3 \\
(Spannweite in Jahren) & $(3-33)$ & $(1-33)$ \\
\hline
\end{tabular}

Die Zeitangaben entsprechen dem Stand von 2017. Die Daten bezüglich der Niederlassung berücksichtigen praktische Ärzte ohne Facharztqualifikation, jedoch keine Ärzte in Weiterbildung; die Angaben zur Facharztqualifikation berücksichtigen keine der beiden Gruppen. Zwei Teilnehmer hatte Katharina Schmalstieg bereits vor dem Interview flüchtig getroffen. 
Alle anderen waren der Interviewenden zum Zeitpunkt der Datenerhebung unbekannt, sieht man von öffentlich zugänglichen Informationen ab (Internetseite der Praxis). Die Interviewlänge betrug zwischen 28 und 72 Minuten bei einer durchschnittlichen Dauer von 42,5 Minuten.

\subsubsection{Praxismodelle der Teilnehmer}

Neun der 17 befragten Ärzte waren in einer Gemeinschaftspraxis beschäftigt. Ein Teilnehmer wurde „ein paar Stunden“ pro Woche vom Vorgänger unterstützt, arbeitete aber vorwiegend ohne ärztliche Hilfe; ein anderer teilte sich die Räume mit einer Naturheilpraxis, in der aber kein weiterer Arzt arbeitete. Die Übrigen waren in einer Einzelpraxis tätig bzw. tätig gewesen, da sich ein Arzt zum Zeitpunkt des Interviews seit acht Wochen im Ruhestand befand. Elf der befragten Ärzte arbeiten mit und zwei ohne Terminvergabe; vier vereinbarten nur zu bestimmten Zwecken mit ihren Patienten Termine, beispielsweise bei längerem Gesprächsbedarf, Kuranträgen, Check Up-, DMP- oder Laboruntersuchungen. Der Einschätzung der Teilnehmer zufolge dauerte der Kontakt mit einem Patienten im Schnitt 14,5 Minuten (mit einer Spannweite von 5-30 Minuten), wobei zwei Ärzte keine Angabe zur Gesprächsdauer machen wollten oder konnten.

\subsubsection{Betreute Patientenpopulation}

Sechs von 17 Ärzten beschrieben ihre Patientenpopulation als überwiegend geriatrisch; einige mit dem Zusatz „multimorbide“, „chronisch krank“ und/ oder „,weniger mobil“. Zwei Teilnehmer gaben an, eine allgemeinmedizinisch gemischte Klientel zu behandeln, wohingegen ein anderer den großen Anteil an Jugendlichen unter seinen Patienten hervorhob. Sechs der Ärzte stellten nicht das Alter, sondern die geographische Lage in den Fokus; so wurden Patienten im kleinstädtischen Bereich im Vergleich zur Großstadt als weniger kritisch und als an eine hausärztliche Führung gewöhnt empfunden. Im ländlichen Bereich wurden viele handwerklich Tätige, wenig Privatpatienten, „katholische“, „,bodenständige“ und „familiär gut integrierte“ Menschen betreut. Hingegen wurden in einer Großstadtnahen Praxis vorwiegend Akademiker behandelt, die Äußerungen des Arztes eher hinterfragen würden. Ein Arzt im Großstadtgebiet beschrieb seine Klientel als wenig Privat-, dafür aber viele sozial schwache und ausländische Patienten. Zwei Teilnehmer sahen die Besonderheiten ihrer Patientenpopulation bedingt durch die Schwerpunkte der jeweiligen Praxis und kümmerten sich nach eigener Einschätzung im Vergleich zu anderen mehr um Asylbewerber, Sucht- und Schwerkranke sowie psychosomatische Patienten. 


\subsection{Kodesystem}

\subsection{1 Übersicht über das Kodesystem}

Im Zuge der Ergebnisauswertung zeigte sich, dass das ,Ausstellen eines BenzoZPrivatrezeptes für Kassenpatienten' als Handlungsstrategie in einem übergeordneten Paradigma fungiert. Dieses in Abbildung 3 dargestellte Paradigma dreht sich um das zentrale Phänomen der Ambivalenz, welches im Folgenden näher erläutert wird.

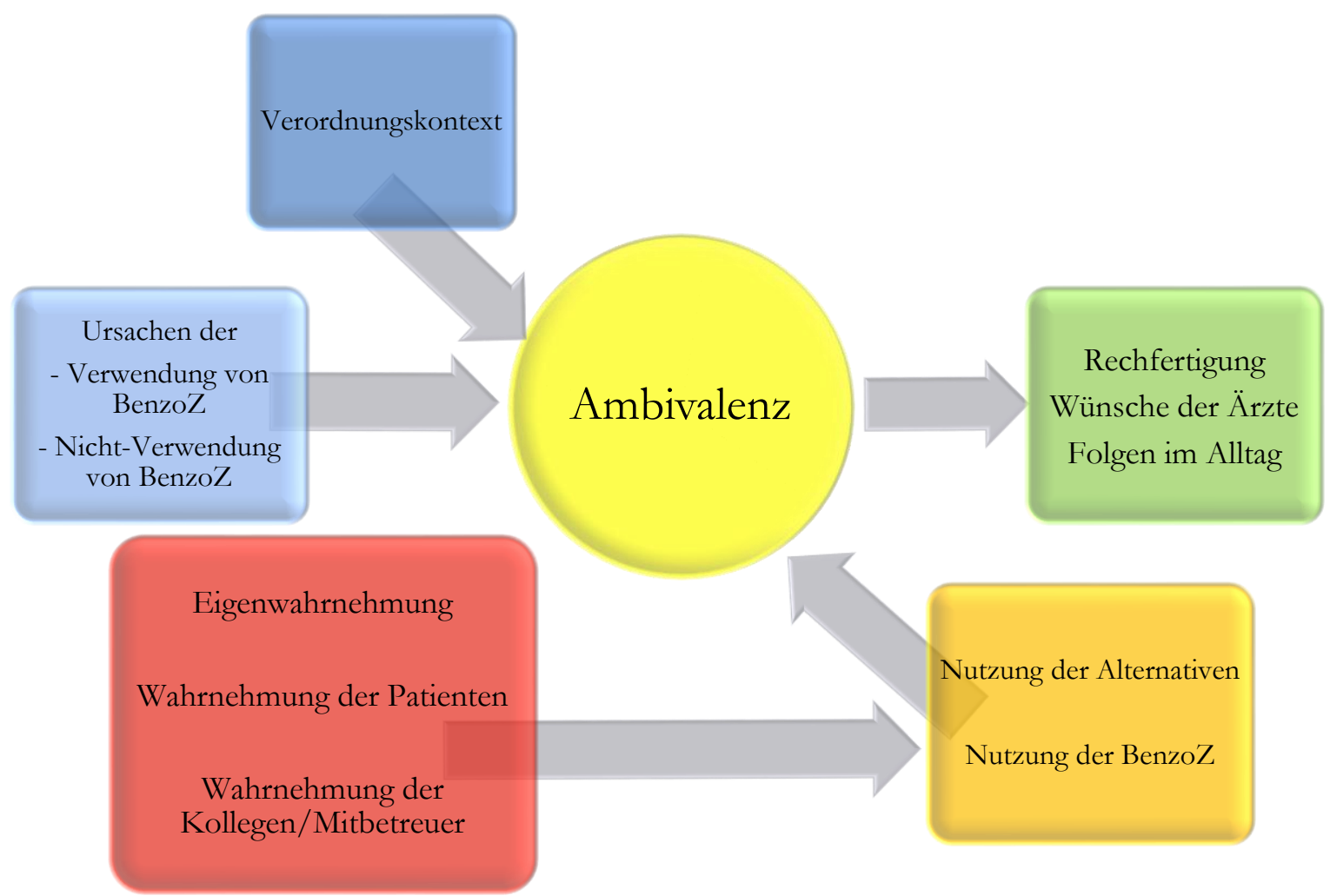

Abbildung 3: Übersicht der Ergebnisse dargestellt an Hand des paradigmatische Modells (Dunkelblau: Kontext, Hellblau: ursächliche Bedingungen, Rot: intervenierende Bedingungen, Orange: Handlungsstrategien, Gelb: zentrales Phänomen, Grün: Konsequenzen)

Eingebettet ist das Phänomen in einen Kontext, der zum einen durch die aktuellen Verordnungs- und Abgabebestimmungen für Benzodiazepine und Z-Substanzen, zum anderen durch das Vorhandensein bzw. das Fehlen von Orientierungshilfen bestimmt wird. Als ursächliche Bedingungen finden sich eine Reihe von Situationen, medizinischen Indikationen und Patienten-immanenten Gründen, die sowohl für als auch gegen die Verwendung von Schlaf- und Beruhigungsmitteln sprechen. Auf Grund des zentralen Phänomens entstehen bei den Befragten Unsicherheit und das Gefühl, sich rechtfertigen zu müssen. Und es ergeben sich sowohl Wünsche als auch praktische Folgen im klinischen Alltag. Die von den Ärzten entwickelten Handlungsstrategien, wie beispielsweise die Nutzung von Alterna- 
tiven zu BenzoZ, erlauben es ihnen, mit diesem Phänomen umzugehen und werden durch die intervenierenden Bedingungen beeinflusst, wie die Eigenwahrnehmung oder die, der Patienten. Die einzelnen Punkte können hierbei als übergeordneten Kategorien verstanden werden, welche unter 5.2.2 ff. detailliert beschrieben werden.

\subsubsection{Verordnungskontext}

Die Ergebnisse zeigen, dass die subjektiv wahrgenommenen Rahmenbedingungen in drei Unterpunkte gegliedert werden können, wie in Abbildung 4 dargestellt. Zum einen schilderten die Teilnehmer einen beobachteten Rückgang der Hypnotika-Verschreibungen, was sich mit den in der Einleitung erwähnten Verordnungszahlen deckt.

Früher war das ja gang und gäbe, dass man jedem das verschrieben hat. Das wurde früber meiner Ansicht nach viel bäufiger verschrieben als heute. (I14, 32)

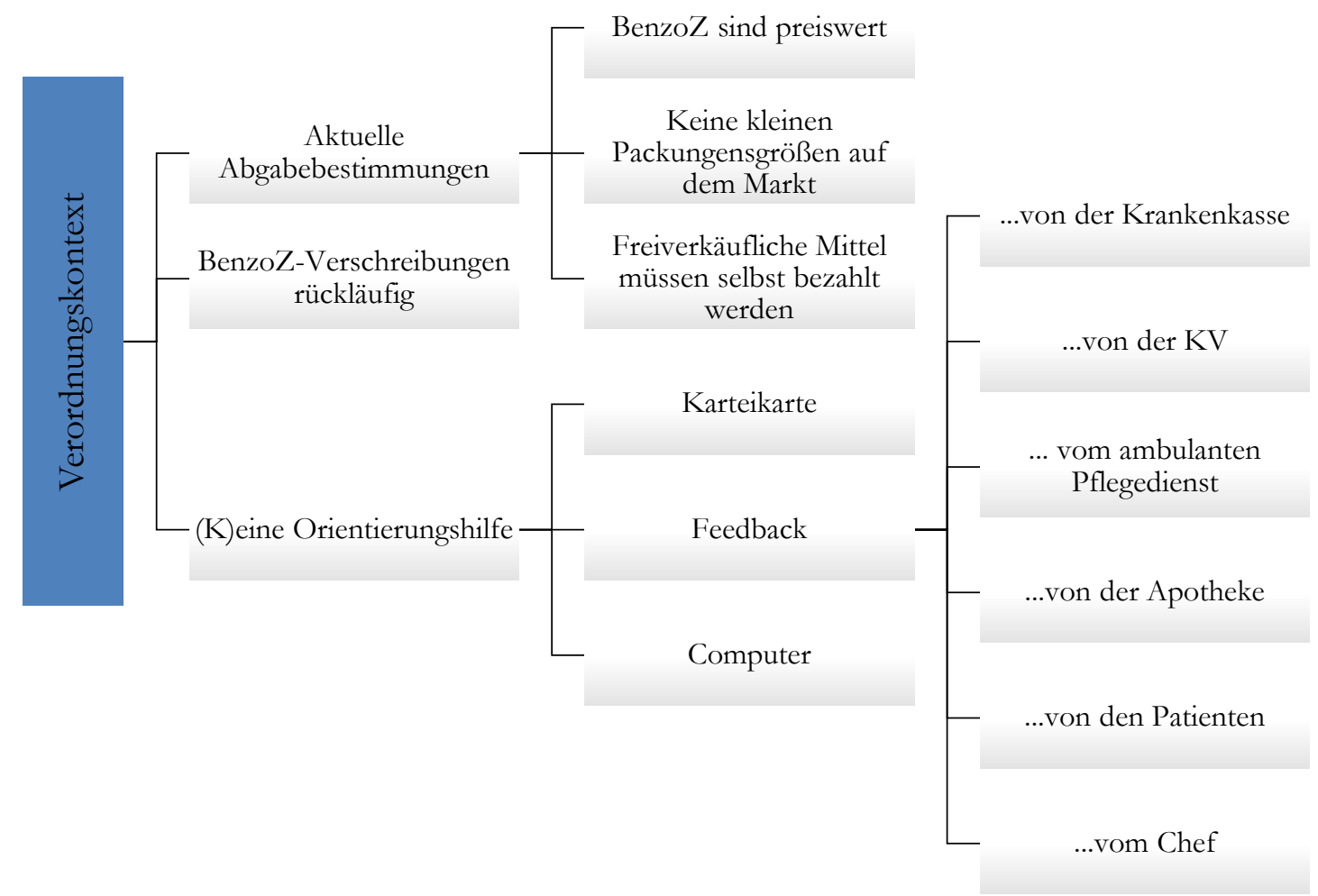

Abbildung 4: Übersicht der Subkategorien des Verordnungskontextes

Ebenso prägen verschiedene Orientierungshilfen den Kontext. Während Karteikarten nur noch vereinzelt genutzt werden, um zu erfahren, wann der Patient das letzte Rezept erhalten hat, verwendeten die meisten Ärzte ein Computer-gestütztes AIS und erhielten so bei der BenzoZ-Verordnung - abhängig vom jeweiligen System - verschiedenste Hinweise, angefangen von pharmazeutischer Werbung, über die Möglichkeit, einer MedikamentenInteraktionsprüfung bis hin zu Verweisen auf die aktuelle Arzneimittelrichtlinie. Allen Systemen gemeinsam war, dass ein Hinweis vom Arzt ignoriert bzw. übergangen werden 
konnte. Jedoch führten insbesondere Vorschläge zur Rezeptform zu Unklarheit und einer gehäuften Verordnung mittels Privatrezept.

Also im Computer ist es insofern schwierig, als dass da die Meldung kommt, man soll's nicht auf Kassenrezept verordnen und man muss es ja trotzdem entscheiden. Aber das ist bei sebr vielen Medikamenten so, ähm. (I2, 40)

Und ja, es kommt ja bei unseren Verordnungen immer schon-gibt uns ja unser EDV-System ein, dass man das eigentlich nur 4 Wochen verordnen darf auf'm Kassenrezept. Und dann nicht darüber hinaus. Ja, da machen wir dann nur bei manchen 'ne Ausnabme und ganz. wenige Zopiclon, wo man denkt, da steht medizinisch begründet auch 'ne schwerere Störung binter, als jetzt nur 'ne Befindlichkeitsschlafstörung. (I7, 48)

Der überwiegende Anteil der Teilnehmer hatte noch nie Feedback von den Krankenkassen oder kassenärztlichen Vereinigung bezüglich Hypnotika-Verordnungen erhalten; einige vermuteten, dass das der Tatsache geschuldet sei, dass Benzodiazepine und Z-Substanzen preiswerte Medikamente sind. Nur ein Arzt gab an, dass er einmalig diesbezüglich Informationen von der KV zu geschickt bekommen hatte. Gelegentlich wurden einige Ärzte von Apotheken über das Vorhandensein von Doppelverordnungen durch andere Kollegen informiert, oder vom Pflegedienst über nicht eingenommene Tabletten, die im häuslichen Umfeld eines Patienten gefunden wurden. Da bis auf eine Person alle ihre Weiterbildung zum Facharzt abgeschlossen hatten und selbstständig arbeiteten, gab es in der Regel kein Feedback eines Vorgesetzten. Des Weiteren gaben zwei Ärzte an, dass sie auch von Patienten wenig Rückmeldung über den Behandlungserfolg mit Benzodiazepinen, Z-Substanzen oder pflanzlichen Alternativen erhielten.

\subsubsection{Ursächliche Bedingungen}

Die ursächlichen Bedingungen lassen sich grob in Ursachen für die Verwendung und für die Nicht-Verwendung von BenzoZ unterteilen.

\subsubsection{Ursachen der Nicht-Verwendung von BenzoZ}

Hier zeigt sich eine Dreiteilung in situations- und indikationsbezogene sowie durch das Patientenverhalten beeinflusste bzw. Patienten-immanente Ursachen. Die Teilnehmer vermieden die Verwendung von Benzodiazepinen und Z-Substanzen in Situationen, in denen ihnen der Patient unbekannt war - entweder beim Erstkontakt in der Praxis oder im Rahmen der notdienstlichen Versorgung. Forderten die Patienten direkt ein Benzodiazepin oder eine Z-Substanz, führte dies in der Regel zur Ablehnung der Verordnung, ebenso das sofortige Verweigern sämtlicher Alternativen zu BenoZ durch den Patienten oder das Nicht-Einhalten der Spielregeln, also das Abweichen des Patientenverhaltens von der ärztlichen Erwartung.

Und bei so ner ganz, straighten Anforderung „Ich will jetzt nen Schlafmittel“ bab ich dann blockiert und gesagt „Nö, mach ich nicht. “ Ne? Wird so nicht laufen. $(\mathrm{I} 3,16)$ 
Ja, ich hatte vor kurzem einen jungen Mann, einen ehemaligen Drogenabbängigen, der sich auch Benzodiazepinen aufschreiben lassen hat. Wir haben alle Tabletten nachgerechnet, es entsprach ungefähr dem zeitlichen Abstand, das kam bin. Ich wusste allerdings nicht, dass er die Tabletten zuhause sammelt und dann alle paar Wochen mal 'ne ganze Packung auf einmal schluckt. (...) Also der Patient bekommt von uns jetzt überhaupt nichts mehr verordnet. (I9, 152)

Des Weiteren lehnten die meisten der Befragten eine Verordnung an beiden Enden des Spektrums ab und gaben an, sowohl für BenzoZ-naive Patienten, als auch bei bekannter BenzoZ-Abhängigkeit kein Rezept auszustellen.

Eine Übersicht der indikationsbezogenen Ursachen kann Abbildung 5 entnommen werden. Der In-vivo-Ausdruck „,harmlose Geschichten“ wurde nicht weiter definiert und steht zusammen mit den nicht durch andere Ursachen bedingten Schlafstörungen (reine Insomnie) den schwerwiegenderen Diagnosen gegenüber, die dann eventuell auch den Einsatz von BenzoZ rechtfertigen (s. Abb. 6).

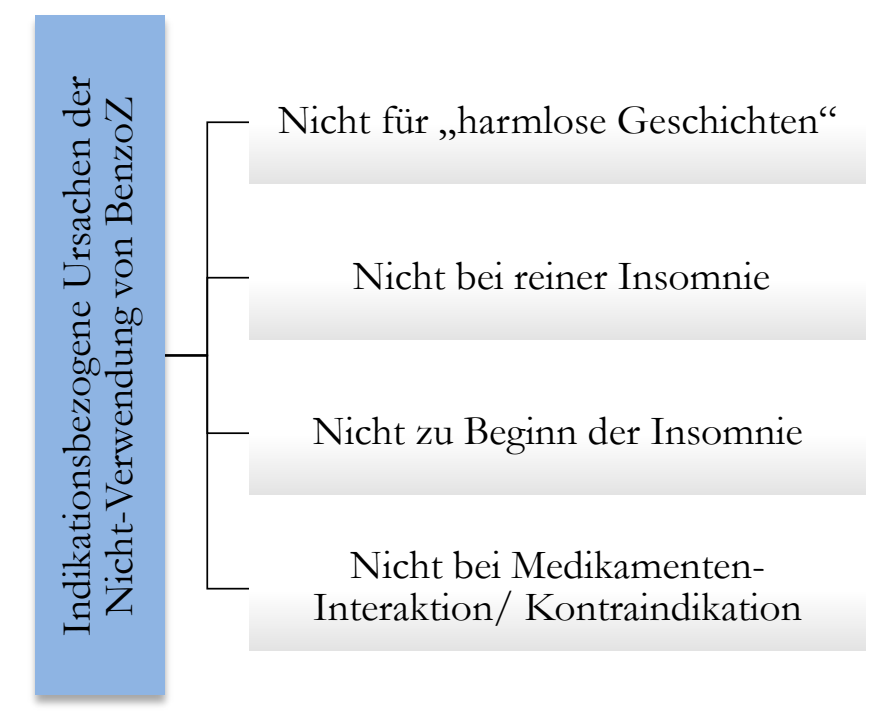

Abbildung 5: Subkategorien der indikationsbezogenen Ursachen der Nicht-Verwendung von BenzoZ

\subsubsection{Ursachen der Verwendung von BenzoZ}

Die Gründe für die Verschreibung von Benzodiazepinen und Z-Substanzen lassen sich ebenso, wie die unter 5.2.3.1. genannten, in situations-, indikations- und patientenbezogene Ursachen unterteilen. Ein weiterer und wesentlicher Faktor ist jedoch auch die Resignation, die sich bei vielen der Befragten im Umgang mit BenzoZ eingestellt hat.

Es gibt welche, die kriegen sie nicht dauerhaft entrogen und das ist so etwas, da muss man sich erst mit abfinden als Arzt in seinem-im Laufe seines Lebens, weil man immer so denkt, ach, mit genügend Ein- 
satz und, und Zuwendung und was weiß ich was und du hängst dich da rein und du kriegst das hin und, äh, ja, da klärt man langsam auch so'n bisschen ab, ne. $(113,19)$

Insbesondere in Krisensituationen, wie beispielsweise der Tod eines nahen Angehörigen, sahen die meisten Ärzte den Einsatz eines Medikamentes gerechtfertigt; hierbei wurde oft auch auf die Kurzfristigkeit der Verordnung hingewiesen. Diese ist in den meisten Fällen als überbrückende Maßnahme gedacht - bis sich die Situation durch den zeitlichen Abstand zum auslösenden Ereignis wieder gebessert hat. Jedoch gab ein Teilnehmer an, Benzodiazepine auf Grund der sozialen Situation des Patienten längerfristig zu verschreiben.

Der [Patient] ist also obdachlos und äbm, lebt also in der [Name], das ist hier die Obdachlosenunterkunft, auf einem Zimmer mit Säufern und ähm in dieser äh, Umgebung ist- und in dieser Lebenssituation, wo die Leute mehr oder weniger auf der Straße leben, ist an eine- so etwas wie ein BenzodiazepinEntzug nicht dran zu denken. Dazu müssen geordnete Lebensverbältnisse da sein. Insofern ist das jemand, dem ich im Augenblick das verordne. (I1, 110)

Bezogen auf die Patienten gaben die Ärzte an, dass ältere Patienten eher ein Benzodiazepin oder eine Z-Substanz erhalten würden als Jüngere. Dies wurde damit begründet, dass insbesondere das Problem der Abhängigkeit im Alter eine untergeordnete Rolle spielen oder im Fall einer bereits bestehenden Abhängigkeit - ein Entzug die Lebensqualität zu stark beeinträchtigen würde, oder dass insgesamt die Vorteile der Verordnung die Nachteile überwiegen würden.

Wenn jetzt ein Patient 70, 75 ist, und kann nicht schlafen und nimmt ne Viertel Tablette und kommt damit gut zurecht, warum soll er das nicht nebmen, ne? Wenn es jetzt ein jüngerer Mensch ist, würde ich es nie anwenden. Aber der Ältere hat nichts zu verlieren außer Schlaf und Lebensqualität. Und das ist halt wirklich sehr gut eingesetat, finde ich. (I16, 75)

...und, äbm, bei älteren Patienten bin ich also balt entschieden großä̈giger, weil ich mir sage, wer mit 80 noch abhängig wird, dem habe ich nicht mehr viel geschadet, aber der hat Lebensqualität gewonnen, wenn er gut geschlafen hat. $(\mathrm{I} 13,7)$

Als Ursache der Schlafstörung sahen die Befragten oft eine Missachtung von Schlafhygiene-Regeln oder unrealistische Erwartungen ihrer Patienten an den Schlaf, wobei das Identifizieren dieser Ursache nicht immer einen Verzicht auf Medikamente bedeutete.

Und nun ist es naturgemäß so, dass die Menschen weniger Schlaf brauchen im hohen Alter. Das verstehen viele aber nicht, ja? Dass das so ist. Und können es nicht akzeptieren. (I1, 98)

Aber bei den Älteren ist das halt wirklich so, dass die eben mittags sich drei Stunden binlegen und sich wundern, dass sie nachts nur zwei Stunden schlafen können. Und dann nehmen sie ein Schlafmittel und dann sind sie den ganzen Tag müde und- (...) Macht so'ne Kreislaufbewegung. Das ist dann natürlich immer das, wo ich sage, okay, da muss man natürlich irgendwann versuchen, einzugreifen. (...) Wobei dann eben, wie gesagt, der alte Patient- man auch sagt: Okay, was soll's jetzt noch, ne? Aber letztendlich ist das halt ne kurafristige Therapie und das verstehen die Meisten halt nicht. $(\mathrm{I1} 7,16)$ 
Ein Arzt gab an, für bereits abhängige Patienten Benzodiazepine weiter zu verordnen. Dies steht im Widerspruch zu den Aussagen einiger anderer Teilnehmer, gerade bei bestehender oder vermutetet Abhängigkeit Verschreibungen zu vermeiden.

Es gibt Patienten, bei denen komm ich nicht drum rum, weil die eben abbängig sind, ne? Und da verwende ich dann im Allgemeinen das Benzodiazepin, mit dem sie kommen, ne? (I1, 26)

Die indikationsbezogenen Ursachen, die für die Verwendung von BenzoZ sprechen, sind in Abbildung 6 dargestellt. Hierzu zählen vor allem eine Reihe von psychiatrischen Erkrankungen sowie Patienten mit schweren organischen Erkrankungen - insbesondere Palliativpatienten. Die meisten Ärzte, die psychiatrische Erkrankungen als Grund für eine BenzoZVerordnung angaben, spezifizierten diese nicht näher. Andere unterschieden die angegebenen Krankheitsbilder, wobei Belastungs- oder Anpassungsstörungen nach einem auslösenden Ereignis zu Traumatisierungen zusammengefasst wurden.

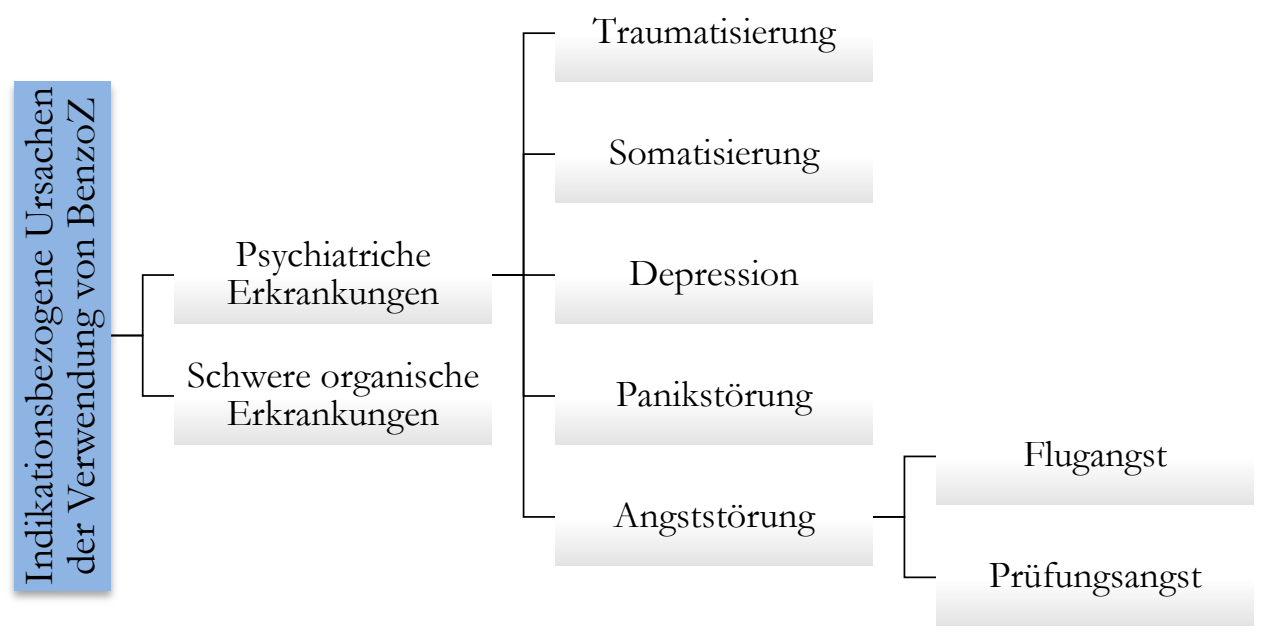

Abbildung 6: Subkategorien der indikationsbezogenen Ursachen der Verwendung von BenzoZ

\subsubsection{Ambivalenz als zentrales Phänomen}

Das zentrale Phänomen der BenzoZ-Verschreibung im hausärztlichen Setting ist die Ambivalenz.

Bezogen auf die Verordnung von Benzodiazepinen und Z-Substanzen lässt sich feststellen, dass diese Medikamente und deren Verwendung bei den Studienteilnehmern widersprüchliche Gefühle, Wünsche und Beurteilungen hervorriefen: Auf der einen Seite möchten die interviewten Ärzte ihren Patienten helfen, indem sie ihnen eine Therapie anbieten, die von beiden Seiten - Arzt und Patient - als wirksam empfunden wird. 
$\mathrm{Ne}$, also es ist nicht so, dass, äh, ich mir vorstelle, als Arzt hätte ich obne Benzos oder verwandte Stoffe arbeiten können, weil, also es ist doch wirklich 'n' $n$ echter Nutzen und es ist auch ein echter Bedarf da und der Missbrauch, der steht immer auf'n andern Blatt, ja, das ist- das haben wir ja nun bei vielen wissenschaftlichen Themen, ne. Da ist ja nicht nur die Medizin gefragt. (I13,75)

Auf der anderen Seite möchte sie die Nebenwirkungen der Benzodiazepine und ZSubstanzen, insbesondere die durch einen längeren Konsum resultierende Abhängigkeit, vermeiden.

Ich möchte meinem Patienten belfen und ich möchte den Eindruck haben, dass ich was Positives für ihn erreiche. $(\mathrm{I} 3,108)$

Also die Effektivität [der Alternativen zu Benzodiazepinen und Z-Substanzen] schätze ich gering ein. Irgendwelche Versuche jemanden, der tatsäcblich sebr realistische Schlafstörungen schildert, ähm, damit wirklich effektiv zu behandeln, sind eher gering. $(\mathrm{I} 3,48)$

Also prinzipiell, ähhm, gibt es ne relativ hohe Hemmschwelle Benzodiazepine zu verwenden. (I3,10)

Also was natürlich gan₹ krass da ist, ist die Befürchtung von Abbängigkeit. Abbängigkeit, die qu einem gesteigerten Konsum führt und hinterher dann eventuell zu so hobem Konsum, dass es erhebliche Schwierigkeiten gibt einen Patienten davon wieder runter qu bekommen. (I3, 26)

Des Weiteren sahen sich die Teilnehmer zum einen als diejenigen, die über Entscheidungsgewalt bezüglich Therapieinitiierung- und -fortführung verfügten und erlebten sich zum anderen auch als machtlos.

Und das zweite sind Patienten bei denen wir tatsächlich, ja Schlafstörungen behandeln müssen, also selber aktiv werden müssen, äh, zu entscheiden, ob sie ne Verordnung von Benzodiazepinen oder anderen Schlafmitteln brauchen. (I2, 7)

...die [Patienten] haben ibre Abbängigkeit seit vielen Jahren und haben gar keine Motivation sich entwöhnen zu lassen. Und dann wird man ja zum Mitläufer, dann muss man verordnen, weil es völlig klar ist, dass die ansonsten ne Entrugssymptomatik bekommen. (I2, 9)

\subsubsection{Handlungsstrategien}

Um mit der Ambivalenz umzugehen, sie zu bewältigen oder darauf zu reagieren, nutzten die Studienteilnehmer eine Reihe von Strategien, die sich grob in die Verwendung von medikamentösen und nicht-medikamentösen Alternativen auf der einen Seite und in die Verwendung von BenzoZ auf der anderen Seite unterteilen lassen. Beide Punkte lassen sich jeweils in untergeordnete Handlungsstrategien aufteilen. Geht es bei der ,Nutzung der Alternativen' vorwiegend um die BenzoZ-Vermeidung, dient die Handlungsstrategie ,Nutzung der Benzodiazepine und Z-Substanzen' mit ihren untergeordneten Strategien der BenzoZ-Kontrolle. 


\subsubsection{Nutzung der Alternativen}

Die am meisten verwendete und häufig zuerst genutzte nicht-medikamentösen Intervention war die Beratung, insbesondere über Schlafhygiene. Zwei Ärzte empfahlen ihren Patienten zudem Entspannungsübungen, progressive Muskelrelaxation oder autogenes Training; zwei weitere sahen die Psychotherapie als gute nicht-medikamentöse Behandlungsalternative oder ergänzende Maßnahme an, gaben aber zu bedenken, dass oft kein Psychotherapeut verfügbar wäre.

Möglicherweise war dies ein Grund dafür, dass neben der Beratung viele medikamentöse Alternativen zum Einsatz kamen. Ein weiterer könnte die Wahrnehmung der Ärzte sein, dass Patienten ein Medikament zur Therapie ihrer Schlafstörung bevorzugen.

Also Patienten verlangen ja oft ein Medikament zur Behandlung, das ist irgendwie leichter als was selber zu tun. Dann als pflanzliche Mittel erstmal die Empfehlung Hopfen, Melisse, äbm, auch Johanniskraut, ähm, es gibt diese Mischpräparate auf pflanzlicher Basis, die man verordnen kann. Das ist der erste Schritt. (I2, 19)

Wie aus dem Zitat deutlich wird, wurde zunächst die Gruppe der pflanzlichen Mittel als medikamentöse Behandlungsalternative genutzt, insbesondere Baldrian wurde oft empfohlen. Des Weiteren gebrauchten die Befragten homöopathische Mittel, Melatonin oder Antihistaminika, wie beispielsweise Diphenhydramin oder Doxylamin, auch Neuroleptika, wie Promethazin oder Melperon, wurden von einigen Ärzten verschrieben. Ein Teilnehmer empfand das Antikonvulsivum Pregabalin als hilfreich. Die neben den pflanzlichen am meisten verwendeten Mittel waren jedoch die Antidepressiva, wie Mirtazapin, Doxepin, Amitriptylin oder Opipramol. Einige der Ärzte berichteten, hiermit gute Erfahrungen gemacht zu haben, da viele Patienten zusätzlich zu ihrer Schlafstörung andere Erkrankungen hatten, wie eine Depression oder Angststörung, oder die Insomnie im Rahmen der Depression oder Angststörung aufgetreten war. Jedoch gab es auch kritische Stimmen bezüglich der Off-Label-Verwendung.

Aber ob das immer dann so richtig ist, auch gerade wenn man's jetzt dann nur als Schlaftablette einnehmen würde, ist eigentlich auch nicht korrekt, ne. (...) Ne, man kann dann den Leuten 'ne depressive Tönung einreden, aber ich denke auch da muss man's immer mal wieder hinterfragen, ob das sinnvoll ist. $(\mathrm{I} 10,30)$

Und obwohl einzelne Teilnehmer über sehr gute Erfahrungen berichteten, wurden Alternativen im Vergleich zu Benzodiazepinen und Z-Substanzen oft als nicht effektiv wahrgenommen.

Alternative in dem Sinne, es gibt ja keine, meine ich, keine genauso gut wirksamen mit weniger Nebenwirkungen. Das wär ja schön. Da träumen wir ja von. Sondern es gibt welche mit weniger Nebenwirkungen, die dann aber eben auch nicht so viel Hauptwirkung haben. (I13, 67)

Des Weiteren hätten Patienten in vielen Fällen die freiverkäuflichen Mittel bereits erfolglos ausprobiert, bevor sie den Hausarzt aufsuchen würden. 


\subsubsection{Nutzung der Benzodiazepine und Z-Substanzen}

Diese Handlungsstrategie zeigte sich in vielen unterschiedlichen Ausprägungen. Eine Darstellung dieser Dimension ist in Abbildung 7 zu sehen.

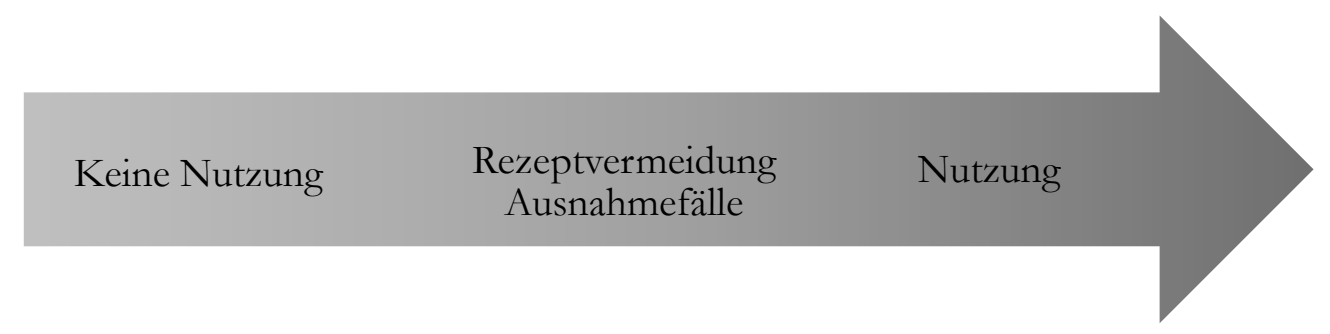

Abbildung 7: Die Dimension der Handlungsstrategie ,Nutzung der BenzoZ‘

Sollte oder wurde ein Rezept ausgestellt, nutzten die Ärzte verschiedene untergeordnete Handlungsstrategie; Abbildung 8a zeigt eine Übersicht, insbesondere der im Vorfeld der Verschreibung verwendeten.

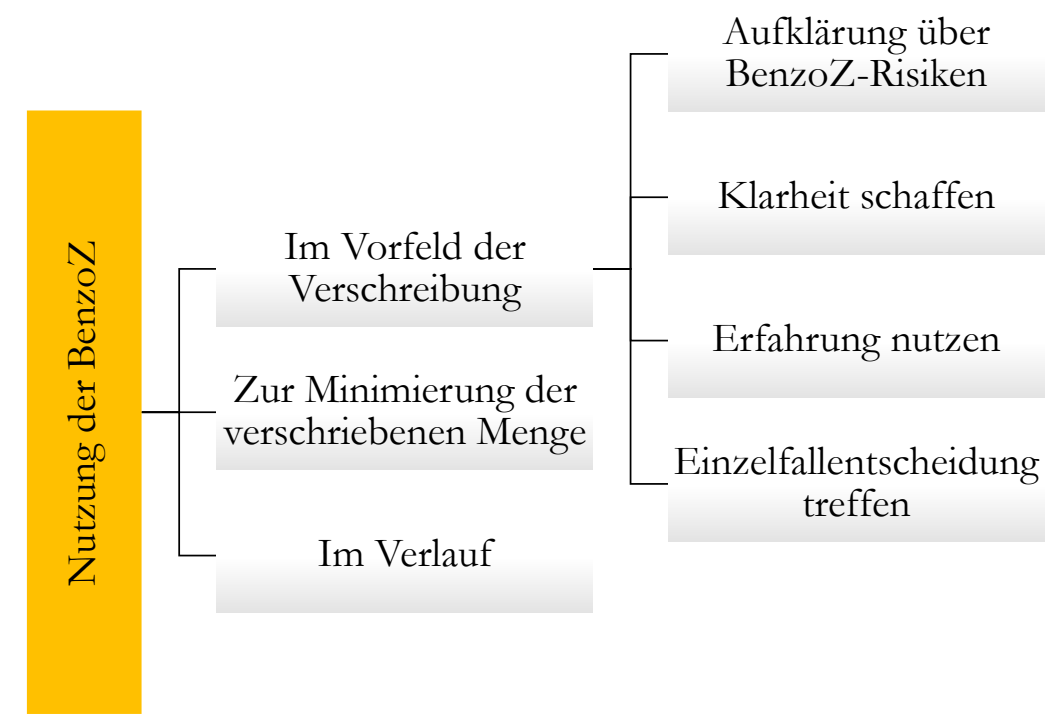

Abbildung 8a: Übersicht der im Vorfeld genutzten Strategien. Stand eine potentielle Verordnung eines Benzodiazepins oder einer Z-Substanz zunächst einmal nur im Raum, bedienten sich die Befragten verschiedener Vorgehensweisen.

Wichtig war es den Teilnehmern, im Vorfeld insbesondere Klarheit zu schaffen. Dies wurde durch folgende untergeordnete Punkte erreicht:

○ Ursachenforschung betreiben

- Medikationsplan prüfen

- Grenzen setzen

- Eigenen Algorithmus schaffen

- Sicherheit gewinnen durch Dokumentation 
Insbesondere herauszufinden, warum ein Patient den Wunsch nach einem Schlafmittel hat, Grenzen abzustecken, und das Kreieren eines persönlichen Algorithmus verhalf den Ärzten zu mehr Klarheit. Jedoch könnte die Überweisung an den Facharzt zu einem bestimmten Zeitpunkt nicht nur als eigner Algorithmus angesehen werden, sondern auch zur Minimierung der verschriebenen Menge dienen (Vergleiche Abb. 8c).

...ja, gut, dann brauche ich nen neurologischen Befund oder irgend so was und geb dem ne Überneisung in die Hand. Und beim dritten Mal sage ich dann spätestens: obne Befund gibt's nix. (I17, 90)

Des Weiteren spielte im Vorfeld einer Verordnung die Erfahrung im Umgang mit Patienten und BenzoZ eine wichtige Rolle.

So am Anfang hatte ich noch Mitleid, so die ersten zwei Jahre nach der Niederlassung. Aber jetzt- man merk.tja, man wird ja ausgenutzt. (I16, 240)

Letztendlich betonten viele der Interviewten, dass die Verschreibung eines Hypnotikums immer eine Einzelfallentscheidung sei.

Zu den Strategien, die im Verlauf genutzt wurden (s. Abb. 8b), zählen vor allem die Abgabe der Verantwortung - zum einen an den Patienten, der die Verordnung trotz Aufklärung über die Risiken gewünscht hat; zum anderen an ärztliche Kollegen, zu denen der Patient überwiesen wird und die über eine (Weiter-)Verordnung entscheiden sollen. Ähnlich verhält es sich mit der Abweisung der Schuld, sollte ein Patient BenzoZ-abhängig geworden sein: Der Patient wollte das Medikament oder ein anderer Arzt hat es verordnet und somit die Abhängigkeit erzeugt. Um die entstandene Problematik möglichst gering zu halten, gaben einige Teilnehmer an, so wenig wie möglich zu verordnen und/oder darauf zu achten, dass der jeweilige Patient nur ein Präparat und nicht etwa mehrere oder zusätzlich Alkohol konsumierte. Oft diente die die Weiterverordnung im Verlauf auch der Konfliktvermeidung, da das erneute Aushandeln der Verordnungsmenge und -dauer von einigen Befragten als anstrengend empfunden wurde.

Aber man hat auch immer so das Gefühl, das wird dann auch immer sehr viel geschachert, 'ne, so: „'ne, möcht' ich das? Neee, und das reicht doch nich'. Und jaa, ich hab jetzt doch drei verloren ". Und dann weiß man auch schon Bescheid. (I6, 40) 


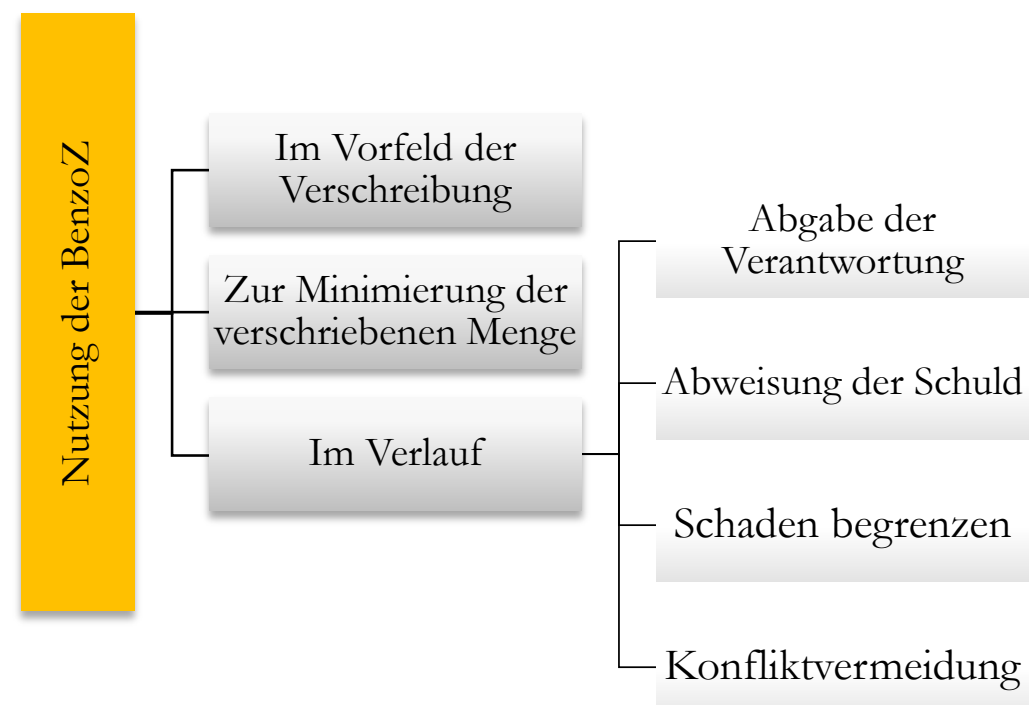

Abbildung 8b: Darstellung der im Verlauf verwendeten Strategien, wenn bereits ein Benzodiazepin oder eine Z-Substanz eingenommen wurden.

Besonders interessant mit Blick auf die Forschungsfrage sind die Strategien, die zur Minimierung der verschrieben BenzoZ-Menge verwendet wurden; eine diesbezügliche Übersicht ist in Abbildung 8c dargestellt. Der überwiegende Teil der Befragten verordneten ausschließlich kleine Packungsgrößen, riet den Patienten die Tabletten zu halbieren oder zu vierteln und versuchte durch positive Suggestion den Effekt kleinster Wirkstoffmengen zu unterstreichen.

Man muss ja immer gucken, dass man einen Placebo-Effekt da mitnimmt, ne, damit die [Patienten] nicht gleich wieder mehr einfordern. (...) Das [Verordnen von Benzodiazepinen und Z-Substanzen], ähm, also das versuche ich schon relativ wichtig zu machen, damit die binterher nicht, ähm, dann so schnell so viel mehr einfordern. Also das-Ich weiß nicht, ob das Einbildung ist, ob es da irgendwie Untersuchungen für gibt, aber ich versuche halt dann, diese, diese Dosissteigerung so lange, wie möglich, nach binten raus zu zögern, da drüber, dass ich immer sage „Oh, das wirkt aber gut", und so, wie man das dann versucht, irgendwie abzupuffern, weil man ja weiß, ähm, die werden es jetżt auf Jahre brauchen. (I8,76) 


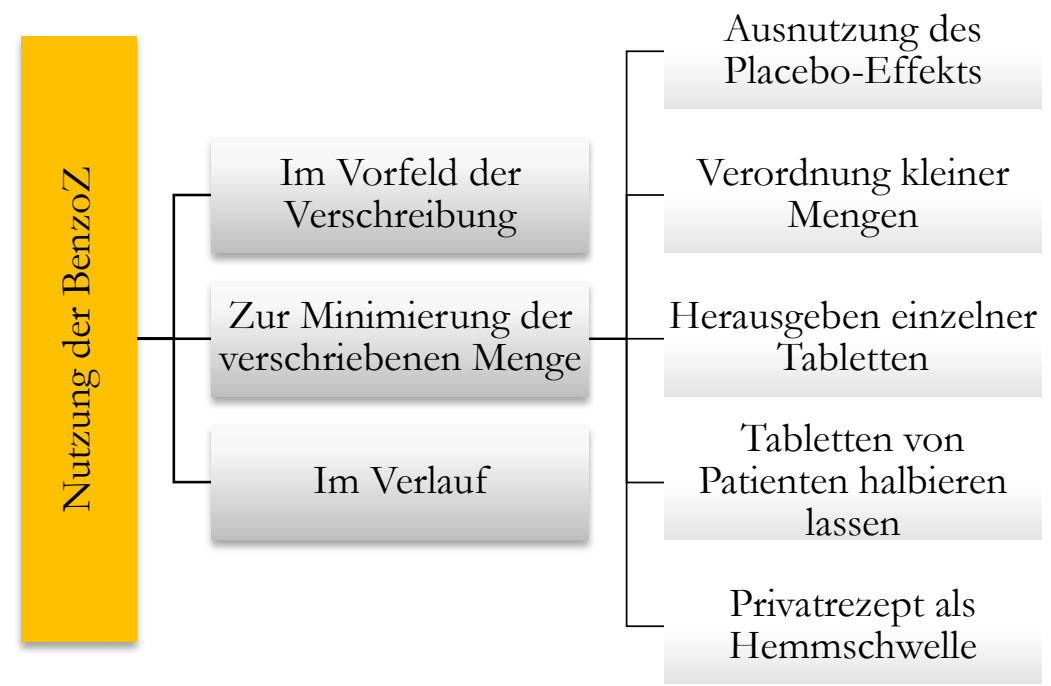

Abbildung 8c: Übersicht der Strategien, die zur Minimierung der verordneten Menge an Benzodiazepinen und Z-Substanzen dienen

Das Herausgeben einzelner Tabletten aus einem sich in der Praxis oder im HausbesuchsKoffer befindlichen Vorrats fand vorwiegend bei unvorhergesehenen Ereignissen und Krisen, z. B. einem Trauerfall statt, aber auch im Notdienst, wenn unbekannte Patienten behaupteten ein BenzoZ als Dauermedikation zu erhalten, dies aber nicht nachgeprüft werden konnte. In diesem Zusammenhang wurden häufig, wie unter 5.2.2 bereits erwähnt, die auf dem Markt erhältlichen Packungsgrößen angesprochen, die eine Verordnung weniger Tabletten unmöglich machen. Letzteres wurde durch die Autorin näher recherchiert und ist unter 5.5.4 dargestellt.

Ebenfalls wurde mit der Verschreibung mittels eines Privatrezeptes versucht, die vom Patienten eingenommene Menge möglichst gering zu halten, wie unter 5.3 ausführlich erörtert wird.

\subsubsection{Intervenierende Bedingungen}

Intervenierende Bedingungen erleichtern, hemmen oder beeinflussen die Auswahl der Handlungsstrategien und haben somit Einfluss darauf, wie die Teilnehmer der Studie mit der Ambivalenz umgehen bzw. auf sie reagieren. Die Ergebnisse zeigen, dass sich die intervenierenden Bedingungen in drei übergeordnete Kategorien unterteilen lassen: die Eigenwahrnehmung, die Wahrnehmung des Patienten und die, der Kollegen und Mitbetreuer.

\subsubsection{Eigenwahrnehmung}

Die Eigenwahrnehmung eines Teilnehmers umfasst sowohl seine Sicht auf Schlafstörungen als auch auf Medikamente. Dabei ist es zum einen entscheidend, ob jemand Schlafstörungen als Problem wahrnimmt oder eher als Lappalie; zum anderen, ob Medikamente als 
wirkungslos oder wirkungsvoll bzw. als nebenwirkungslos oder nebenwirkungsvoll eingestuft werden.

(...) [bei der Fortbildung] ging es nämlich auch mal um Schlaf und, ähm, das bat mir-diese Geschichten mit der Schlaflosigkeit, wie quälend das ist und so, das war mir nicht so sehr bewusst, ne. Und wie wichtig das ist, zu schlafen. $(\mathrm{I15}, 120)$

Ebenfalls ist entscheidend, wie sicher sich jemand im Umgang mit Benzodiazepinen und Z-Substanzen fühlt und wie er sich in der Rolle des Arztes wahrnimmt - welche Aufgaben, Rechte und Pflichten er sich zuschreibt, ähnlich den von Charles et al. beschriebenen Konsultationsmodellen. So trifft beim informierten Modell der Patient die Entscheidung, beim Shared Decision Model Arzt und Patient, und beim Patriarchalischen Modell allein der Arzt (Charles et al. 1999).

Also Richtlinien sind wirklich nur reine Richtlinien, bei denen ich sage, ich nebme sie als Anregung für eventuelle Fragestellungen, was mache ich denn jetæt. Prinzipiell mache ich eine Verordnung für einen Patienten in der Hauptsache vom Patienten abhängig. Von der Patientensituation und von dem, was mir geeignet erscheint. Also da bin ich, wie soll ich sagen, mein eigener kleiner König, der sagt, in meinem Reich spielt meine Spielregel die erste Geige. Wenn ich mal nicht ganz klar bin darüber, was ich mache, kann ich ja mal gern in die Richtlinie gucken. (I3, 106)

Die Eigenwahrnehmung wird wiederum durch eigene Erlebnisse geprägt. Solche biographischen Einflüsse könnten ebenfalls zum Kontext gezählt werden, also unter 5.2.2 aufgeführt werden, da aber eigene Erlebnisse die Auswahl der Handlungsstrategien einer Person entscheidend prägen, wie die folgenden Zitate zeigen, werden sie im Rahmen dieser Arbeit zu den intervenierenden Bedingungen gezählt.

(...) und tavorsüchtig war ich insgesamt zwei Jahre, äh, und zwar furchtbar süchtig. (...) Und daher weiß ich, wie schlimm sowas sein kann, wenn es noch viel schlimmer ist. Insofern kann ich den Leuten sagen, mach ich - ich sag das auch. Ich war selber mal als junger Mann abbängig von diesem Mist. (I14, 152)

Aber batte jetzt auch davon weder das Gefühl, dass ich jetżt besonders schnell von eingeschlafen bin, noch dass ich am nächsten Tag noch 'n Übergang hatte. Also, ich weiß nicht, ob ich Tavor 'n halbes oder 'n Milligramm genommen hatte. Also, war in Anfübrungszeichen ein bisschen enttäuscht von der Wirkung. Ich dachte, dass wäre so'n richtiger Pumba und das merkt man dann. (I6, 212)

\subsubsection{Wahrnehmung des Patienten}

Im Zuge der Datenauswertung kristallisierten sich mehrere Patientenstereotypen heraus, die die befragten Ärzte mit der Verordnung von Benzodiazepinen und Z-Substanzen in Verbindung brachten, wie in Abbildung 9 veranschaulicht. 


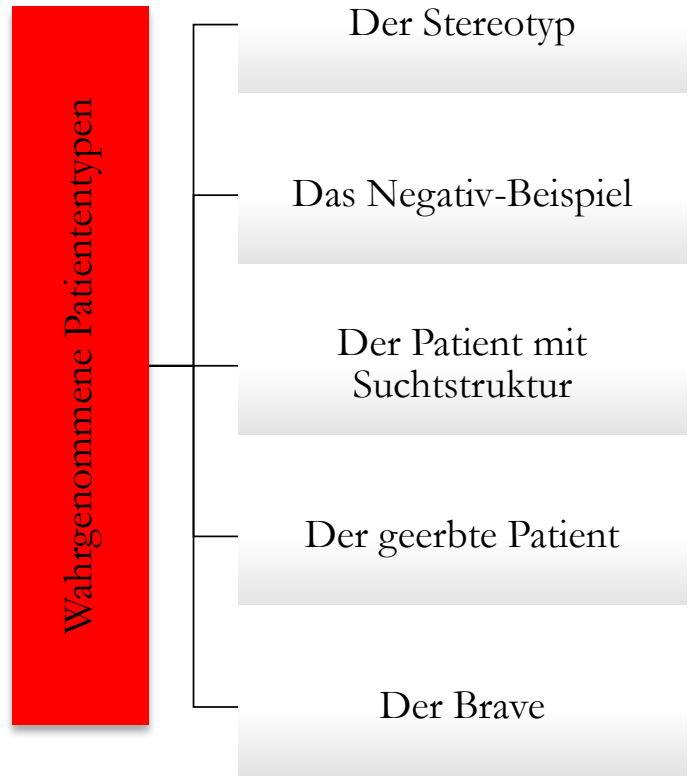

Abbildung 9: Von den Teilnehmern wahrgenommene Patiententypen

Der stereotypische Patient ist alt, meist weiblich und lebt oft im Pflegeheim. Als NegativBeispiel gelten Patienten, die schon lange Zeit Benzodiazepine oder Z-Substanzen konsumieren und/oder von (illegalen) Drogen abhängig sind. In der Wahrnehmung der Befragten ist ein BenzoZ-Entzug hier oft kaum möglich. Diese Fälle bleiben dem jeweiligen Arzt im Gedächtnis; weitere dieser Art sollen vermieden werden.

Also der-Dieser Drogenabhängige da, der die Rezepte gefälscht hat, das war eigentlich so ganz eindrucksvoll. Also der ist mir wirklich kleben geblieben. (I8, 440)

Als Patienten mit Suchtstruktur werden Patienten bezeichnet, von denen angenommen wird, sie würden im Vergleich zu anderen schneller in eine Abhängigkeit geraten. Hier scheinen Verhaltensweisen der Patienten sowie Persönlichkeits- oder sozioökonomische Merkmale eine Rolle zu spielen, bzw. eine in der Vergangenheit oder noch bestehende Sucht nach einem anderen Stoff.

Das hat natürlich-sagen wir mal, es gibt Patienten, von den ich mal so glaube, sie haben von vornerein schon 'ne gewisse Suchtstruktur, das weiß man schon. Das gibt es bei Schmerzmitteln, das gibt es bei äh Berubigungsmitteln, ähm, wenn Sie den das unkontrolliert geben, dann ähm fällt auf einmal auf, der hat in einem Monat ja schon fast 100 Tabletten verbraucht um Gottes Willen. (...) Und, äh, der macht auch falsche Angaben vorsätzlich. Äh, das gibt es auch. Also da muss man ganz vorsichtig sein. Das ist auch wieder das Bild- das ist auch wieder das Bildungsniveau. Das macht der Schlichte, sag ich mal, der schlichte Patient, der macht das, der gebildete Patient würde sowas nie machen, 'ne. (I12,57)

Der Patiententyp, den die Befragten jedoch am häufigsten erwähnten, war der geerbte Patient.

Das sind aber alles Patienten, die ich von jemand anders übernommen habe, die mit ibrer Abhängigkeit praktisch in die Praxis gekommen sind, ne? (I1, 24) 
Das heißt, einige Patienten, die es bei mir jetzt länger nebmen, habe ich übernommen. Ich habe niemanden neu eingestellt auf 'ne Schlafmedikation solcher Art. (I5,28)

Wird ein Patient als geerbt betrachtet, kann die Handlungsstrategie ,Abweisung der Schuld“ angewendet werden. Die Befragten fühlten sich nicht schuldig für die Entstehung der Abhängigkeit eines Patienten, da ein anderer Arzt die Medikation begonnen hatte. Interessant ist, dass es bei diesen Patienten gleichzeitig zu einer Abgabe der Verantwortung für die Unterhaltung bzw. Fortführung der Abhängigkeit kommt. Die Teilnehmer erlebten sich nicht in der Rolle desjenigen, der an der Situation etwas ändern könnte, somit ist die Verantwortung dem vorherigen Arzt oder dem Patienten zu übertragen (Vergleiche 5.2.5.2).

Also, ich hab mehrere Patienten, die sind definitiv Zopiclon-abhängig. Die kriege ich da auch nicht von runter. Ähm, das- die waren aber schon abbängig, bevor ich die Praxis übernommen habe. $(\mathrm{I} 4,6)$

Als brave Patienten wurden diejenigen angesehen, die entweder gar nicht erst nach Schlafoder Beruhigungsmitteln fragen, sich bei Schlafstörungen auf alternative Therapien einlassen oder in den Augen des Verschreibenden sehr geringe Mengen konsumierten. Anzumerken ist, dass der Brave eher ein BenzoZ-Kassenrezept erhält als andere Patiententypen, wie unter 5.3. näher beschrieben wird.

Zudem nahmen Ärzte in ländlichen Praxen ihre Patienten als verlässlich und Probleme wie Drogen- und Medikamentenabhängigkeit als untergeordnet war; auch Doktor-Hopping wurde eher städtischen Regionen zugeschrieben.

(...) also das finde ich tatsächlich, in der Praxis, da wo ich arbeite, auf dem Land, nicht schwierig, weil das ist sehr zuverlässig, die Leute kommen, ähm, das klappt eigentlich da gut. Mit den Diensten merk ich, dass es in den Städten, oder in anonymeren Regionen oder Bevölkerungsteile da nicht so gut klappt und das, ähm, das muss man halt auch ernst nehmen, also das merket man auch, dass man halt verklappst wird sozusagen und dass die bei mehreren Ärzten mehrere Sachen holen. (I2, 115)

Und ich denke, diese Patienten, die umberziehen, um irgendwelche Substanzen vielleicht sogar dann über'n Markt zu kriegen, ja, also die gibt es denke ich schon. Hier auf'm Land haben wir wabrscheinlich-sind gan₹,gut dran. $(\mathrm{I} 10,112)$

Jedoch empfanden die interviewten Ärzte im großstädtischen Bereich den Umgang mit Benzodiazepinen und Z-Substanzen nicht als schwieriger, noch gaben sie an, dass es auf dem Land einfacher sei - möglicherweise, weil sie die unter 5.2.5.2 beschriebene Handlungsstrategie ,Klarheit schaffen' deutlicher umsetzten. Sie schufen ihren eigenen Algorithmus und setzen Grenzen.

Am Anfang ist man ja noch nen bisschen blauäugig, wenn man anfängt, aber wenn man denn konsequent seine Linie durchzieht- Nicht ein Einziger haben wir in den letzten Jahren, der dann die Praxis hier tangiert. Is ja so, dass es sich rumspricht in der Szene, nech und dann gerade hier in [Name Großstadt], nech-dann hat man ständig so'ne Klientel hier. Wenn man da konsequent ist, hat man überhaupt keine Probleme mit Benzodiazepinen und Z-Substanzen. Gar nicht eigentlich. (I16, 4) 


\subsubsection{Wahrnehmung der Kollegen und Mitbetreuer}

Die Teilnehmer dieser Studie nahmen im Zusammenhang mit Benzodiazepinen oder ZSubstanzen drei Gruppen von mitbehandelnden ärztlichen Kollegen wahr: Psychiater, andere Hausärzte und Ärzte im Krankenhaus.

Am unterschiedlichsten wurden Psychiater und deren Verschreibungsverhalten betrachtet.

Das Putzige ist, ich habe die Erfahrung gemacht, dass die [Psychiater] eher panische Attacken kriegen, wenn es darum geht Dauerverordnungen mit Benzodiazepinen zu machen. Die machen das eigentlich nicht. (...) Ich habe aber keine Patienten bis jetzt zum Beispiel von einem psychiatrischen Kollegen gekriegt, die dann mit einer Benzodiazepin-Verordnung zum Beispiel wiedergekommen sind. Zopiclon babe ich schon mal gesehen. Aber Benzo eigentlich nicht. Und, mhm, so im Gespräch, wenn ich also mal einen Kollegen am Telefon hatte und gesagt habe okay, ich habe jetz̧t mal interveniert mit Tavor, weil irgendwo nichts mehr ging, weil die [Patienten] völlig in der Krise waren und keinen Psychiater-Termin hatten, mbm, haben die [Psychiater] mir auch eher den Eindruck vermittelt, dass sie das jetzt zwar tolerieren, aber jetst nicht so begeistert waren. $(\mathrm{I} 3,98)$

Jaa, also ich hab jetz̨t nicht den Eindruck, dass die [Psychiater] viel mehr oder viel weniger als, ähm, wir von uns aus machen, diese Substanzen verordnen. $(17,94)$

Also, da kann man-also ich hab's leider manchmal so gemerkt, dass die Psychiater da ein bisschen kritiklos sind auf dem Gebiet. (...) Ja, es gab durchaus Psychiater, also die so ein bisschen, die zu schnell und zu lange verordnet haben, ja? $(\mathrm{I} 1,114)$

Die einheitliche Meinung der Teilnehmer war, dass der Bedarf an Psychiatern und Psychologen die Verfügbarkeit übersteigt, was in langen Wartezeiten für die Patienten resultiert. Der Großteil der Befragten erlebte die Kommunikation mit den Kollegen als schwierig.

Entsprechend der im Interviewleitfaden formulierten Frage, die die Wahrnehmung der Teilnehmer hinsichtlich mitbetreuender Psychologen, Psychiater und Ärzte für Psychosomatik adressierte, wurden allgemeinmedizinische Kollegen selten erwähnt. Des Weiteren haben die meisten Patienten nur einen Hausarzt, so dass sich eine Zusammenarbeit zwischen zwei Allgemeinmedizinern selten ergibt. Jedoch wurde das Verschreibungsverhalten der Kollegen im Zusammenhang mit übernommenen (geerbten) oder Vertretungspatienten als - im Vergleich zur eigenen Person - „großzügiger“ eingestuft.

Ebenfalls wurden so BenzoZ-Verordnungen im Krankenhaus beschrieben. Jedoch stellte dies nur für einige der Befragten ein Problem in der späteren ambulanten Versorgung dar und wurde im Vergleich zu den aus anderen Praxen geerbten Patienten, selten erwähnt.

Ansonsten wurden dort [im Krankenhaus] sehr großðügig Schlaftabletten verteilt. Es ist ja dann auch zeitlich begrenat und das kann ich dann auch vertreten. $(19,132)$

Das Problem natürlich genauso im Krankenhaus, so nach dem Motto: wenn die im Krankenhaus nicht schlafen können, kriegen die ne Schlaftablette. Dann wird es auch noch drauf geschrieben [in den Entlassungsbrief] und dann wird das einfach weitergemacht. $(117,75)$ 
Ein Teilnehmer reflektierte diesbezüglich sein Verschreibungsverhalten und gab an, sich als Stationsarzt keine Gedanken über die langfristigen Probleme einer BenzoZ-Verordnung gemacht zu haben, die er nun als Hausarzt aber deutlich sehe.

\subsubsection{Konsequenzen}

Auf der einen Seite sind Benzodiazepine und Z-Substanzen wirksame Schlaf- und Beruhigungsmittel, auf der anderen Seite verursachen sie gravierende Nebenwirkungen. Auf Grund dieser Ambivalenz ergeben sich schließlich Konsequenzen, die in mehrere Kategorien unterteilt werden können.

\subsubsection{Rechtfertigung}

Die meisten der Befragten gaben während des Interviews eindeutig ihre Meinung oder Position zum Thema Benzodiazepine und Z-Substanzen wider und rechtfertigten ihr eigenes Vorgehen - teilweise ohne, dass explizit nach dem Warum einer Handlung gefragt wurde.

I: Wenn Sie mal an die Verordnung von Benzodiazepinen und Z-Substanzen, wie, ähm, Zolpidem oder Zopiclon denken, was fällt Ihnen da spontan zu ein? Vielleicht auch im Zusammenhang mit dem ein oder anderen Patientenbeispiel.

P: Ähm, also Benzodiazepine, alsooo Diazepam, Valium, diese ganzen Geschichten, ähm, hab ich ziemlich raus aus meinem Verordnungsverhalten in den letzten Jahren. Es gibt einige Patienten, allerdings, die die drauf bestehen, die schon immer, äbm, das auch genommen haben, wir haben ja die Praxis vor acht Jahren übernommen und es gab halt so festgefahrene, ähm, Wege, die auch nicht zu durchbrechen waren. Hab ein paar Patienten in den letzten Jahren, äh, stationär eingewiesen zum Benzo-Entzug. Es is ja immer lange dann, bis die auch weg sind und diese genannten Z-Substanzen, jaa, verschreib' ich schon öfter mal. Ja, nicht gerne, aber mach ich, äh, ja. (I15,3)

Des Weiteren wurde oft auf die eigene Patientenklientel verwiesen.

Ich habe zu Zeit eigentlich, äh, wenig Patienten, die Benzodiazepine einnehmen, wirklich sehr wenig. $(\mathrm{I} 12,7)$

Zopiclon wird auch ungefähr so bei 10, 12 Patienten bei mir verordnet, die wiederrum sind aber dann in der Regel nicht nur ausschließlich als Schlafmittel eingesetzt. Sondern die baben dann auch noch psychische Nebenprobleme, die das etwas bedingen. Es gibt wirklich nur 1 oder 2, die das quasi aus alter Tradition, da muss ich kurz ausholen, ähm, ich hab die Praxis jetət vor 2 Jahren übernommen (...) (I3, 10)

\subsubsection{Wünsche der Ärzte}

Vier der Befragten gaben keine BenzoZ-spezifischen Wünsche an. Sieben Teilnehmer wünschten sich weniger Einschränkungen durch Vorgaben der Krankenkasse oder des Gesetzgebers, um patientenindividuell und ohne Sorge vor einem Regress eine Entscheidung treffen zu können. 
Also es sollte einfacher sein für ausgewählte Patienten Z-Substanzen auch auf Kassenrezept zu verordnen obne, dass die Gefahr eines Regresses besteht. (I9,121)

Habe ich keine Wünsche. Außer, außer dass ich, ähm, dass ich als Arat, äh, ne freie Entscheidung haben möchte. Und dass ich nicht regressiert werde, wenn ich mal nem Patienten das so oder so aufschreibe. Das ist der allergrößßte Wunsch. $(\mathrm{I} 4,150)$

Jedoch wollten fünf Ärzte mehr Klarheit durch mehr Vorgaben; so sollte das Marktangebot an BenzoZ und die Verordnung auf zwei Wochen eingegrenzt werden. Ebenso sollte die Verordnung über ein Privatrezept geregelt werden.

Also, wie gesagt, dieses, das Privatrezept oder Nicht-Privatrezept, das ist ja so 'nen bisschen, denke ich, so 'nen Graubereich. Vielleicht ähm, ob man das dann nochmal besser regeln sollte. Das man dann, was weiß ich, sagt, man macht das gar nicht. (I10, 109)

Ein Teilnehmer begründete den Wunsch nach strikteren Vorgaben mit einer daraus resultierenden Hilfe, um Langzeitverordnungen abzulehnen.

Dann könnte man auch sagen, okay, du nimmst jetzt-du darfst deinem Patienten böchst so - böchstens, ähm, $5 \mathrm{mg}$ Diazepam am Tag und das für zebn Tage aufschreiben. Das wäre- und länger nicht. Dann bin ich aus dem Schneider. Da kann ich natürlich allen Süchtigen sagen, Leute ihr könnt mich schlagen, ihr könnt mir meine Scheiben einschmeißen, ich darf einfach nicht. So ist es ja immer, ich kann ja mehr. Insofern, wenn es solche richtigen harten, aber durchaus dummen Gesetze geben würde, wäre es natürlich für mich leichter auch einiges abzublocken, denn man schlägt sich schon manchmal mit den beginnend Abhängigen oder schon lange Abhängigen (I14,122)

In neun Interviews und somit am häufigsten wurde der Wunsch nach mehr Unterstützung geäußert, beispielweise durch ein strikteres Verschreibungsverhalten der Kollegen im ambulanten und stationären Bereich, da Patienten, die einmal ein BenzoZ eingenommen haben, dieses möglicherweise erwarten oder bereits abhängig sind. Personelle Unterstützung beim Entzug und kürzere Wartezeiten beim Spezialisten wurden ebenfalls als hilfreich angesehen. Einige sahen die Krankenkasse als die Institution, die BenzoZ-Verschreibungen überwachen und kontrollieren sollte, da dort - so keine Verordnung über ein Privatrezept erfolgt - alle Einnahmedaten eines Patienten zusammenliefen. Finanzielle Unterstützung sollte es in Form besserer Gesprächsvergütung geben. Ein Teilnehmer wünschte sich, dass bei entsprechender Indikation oder beim langsamen Ausschleichen Benzodiazepine auf Kassenrezept verordnet werden könnten. Diese Unsicherheit bezüglich der Rezeptform wird unter 5.3 ausführlich diskutiert.

\subsubsection{Folgen im Alltag}

Als Resultat der Ambivalenz ergeben sich nicht nur Wünsche oder das Gefühl, sich für sein Verhalten rechtfertigen zu müssen, sondern auch praktische Folgen für den Arbeitsalltag. So stehen sich Ideal und Realität oft gegenüber; das Absetzen oder Umstellen von BenzoZ erfolgt aus unterschiedlichen Gründen nicht, sondern die Verordnung wird fortgesetzt - 
trotz des Bewusstseins, dass dies nicht der aktuellen Leitlinie entspricht und mit Nebenwirkungen verbunden ist.

Dann ist auch oft so, dass man denkt, jaaa, eigentlich müsstest du's mal irgendwie umstellen, mal absetzen, müsstest du mit denen mal besprechen. (I6,6)

Ebenfalls wird die Patientenklientel durch das eigene Verschreibungsverhalten beeinflusst, was letztendlich zu einer Selektion führt.

Ich habe versucht, die Drogenabhängigen bier immer rauszuekeln in Anfübrungszeichen. Weil ich auch gesagt habe: mache ich nicht und fertig. Und dann kam se auch kaum noch, weil das Problem in dem Moment, wo man einem nen Rezept gibt, haben Sie gleich-spätestens die zwei nächsten-wieder zwei drinsitzen, die auch was haben wollen. $(117,282)$

\subsection{Benzodiazepine und Z-Substanzen auf Privatrezept}

Wie unter 5.2.5.2 beschrieben, ist das Privatrezept für kassenärztlich versicherte Patienten eine Handlungsstrategie, um auf das zentrale Phänomen der Ambivalenz zu reagieren. Betrachtet man diese Handlungsstrategie aber nicht im Gesamtbild des allgemeinen Umgangs mit Benzodiazepinen und Z-Substanzen, sondern in einem engeren Rahmen, zeigt sich, dass das Ausstellen eines Privatrezeptes auch dazu dient, um das Phänomen oder die zentrale Idee des Kreierens einer Hemmschwelle zu erreichen, wie in Abbildung $10 \mathrm{zu}$ sehen ist. Als übergeordneter Kontext können dabei die bereits dargestellten Ergebnisse, insbesondere die Ambivalenz bezüglich BenzoZ-Verschreibungen, und das Ergebnis der Literaturrecherche zu Einstellungen von Ärzten betrachtet werden, dass Benzodiazepine und ZSubstanz als abhängigkeitsinduzierende Stoffe wahrgenommen werden und die Verwendung oft negativ konnotiert ist. Dies soll im Folgenden nicht erneut erörtert werden, jedoch wird die Unsicherheit bezüglich bestehender Richtlinien als ursächliche Bedingung dargestellt. Beeinflusst wird die Strategie ,Privatrezept ausstellen' durch den Patiententyp, die Diagnose- und Problemdauer, die Substanzklasse sowie die Etablierung einer Routine. Letztere könnte aber auch nicht als intervenierende Bedingung, sondern als Folge interpretiert werden - sowie die Minimierung des Medikamentenkonsums, die Regressvermeidung und die Entlastung des Budgets und der Versichertengemeinschaft. 


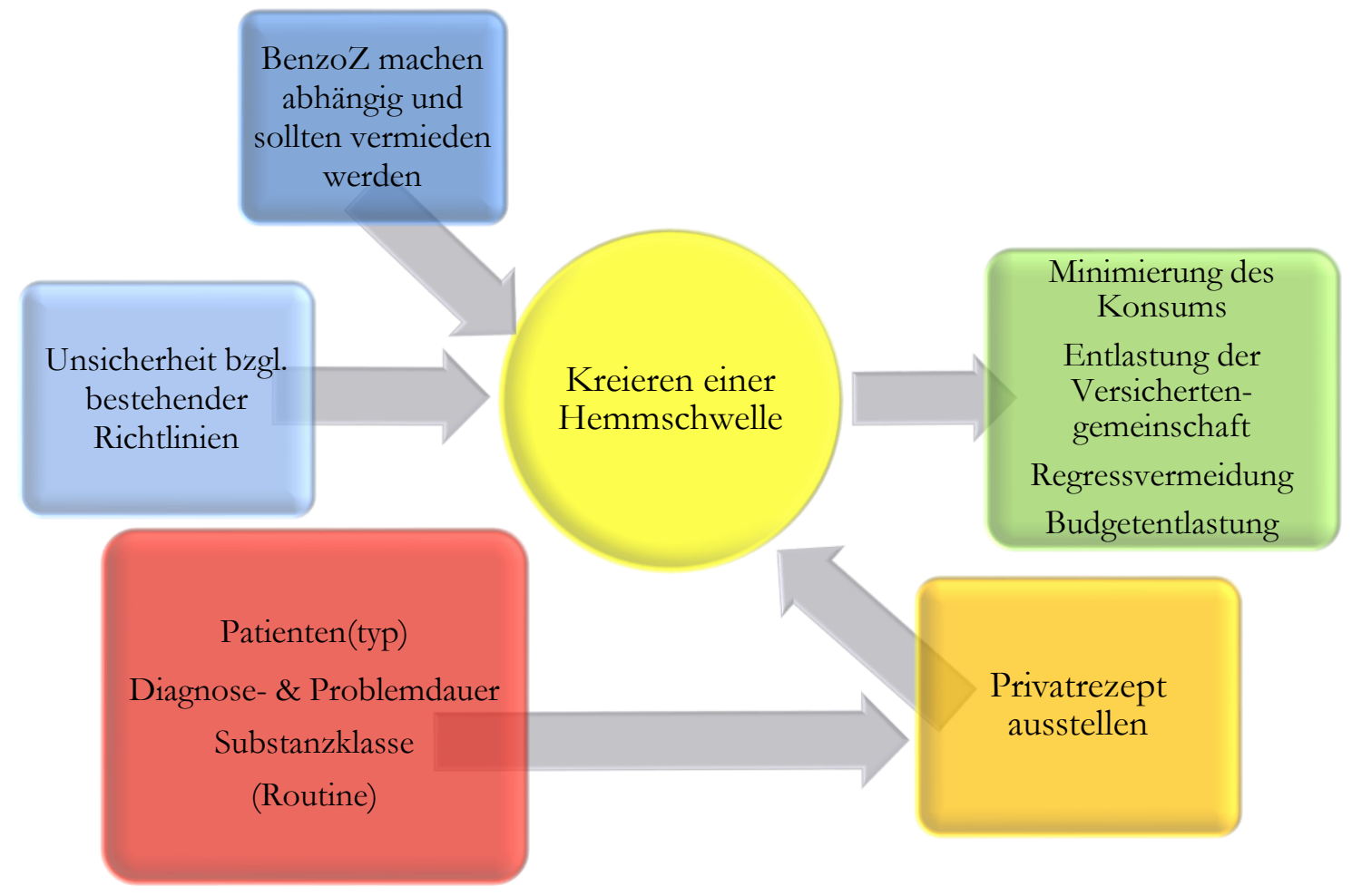

Abbildung 10: Paradigmatisches Modell der BenzoZ-Privatverordnung (Dunkelblau: Kontext, Hellblau: ursächliche Bedingungen, Rot: intervenierende Bedingungen, Orange: Handlungsstrategien, Gelb: zentrales Phänomen, Grün: Konsequenzen)

\subsubsection{Privatrezept auf Grund von Unsicherheit bezüglich bestehender Richtlinien}

Ursächlich für die Wahl der Rezeptform ist die unterschiedliche Interpretation der momentanen Regelungen bezüglich Hypnotikaverschreibungen durch den jeweiligen Arzt und die oft daraus resultierende Unsicherheit. Dabei geht es nicht primär um die Frage, wie Benzodiazepine und Z-Substanzen generell zu verwenden sind. Obwohl neun der 17 Befragten angaben, die aktuelle Arzneimittelrichtlinie (AMRL) gar nicht oder nicht im Detail zu kennen, schilderten alle, dass diese Medikamente kurzfristig und nur im Einzelfall länger genutzt werden sollten. Dies entspricht essentiell den Vorgaben der AMRL, auch wenn die dort erwähnte Frist von vier Wochen nicht explizit genannt wurde. Wesentlich mehr Unklarheit und Unsicherheit besteht bezüglich der Frage, wann und wie lange Benzodiazepine und Z-Substanzen von den Krankenkassen finanziert werden - also per Kassenrezept verordnet werden können - und wann nicht. Beispielweise gab ein Arzt an, das Privatrezept zu nutzen, um große Packungen verordnen zu können, da von den Krankenkassen nur kleine Packungen finanziert würden. Das Privatrezept würde somit den Patienten Geld sparen, da die mehrfachen Rezeptbeteiligungen von fünf Euro die Kosten einer selbst bezahlten Großpackung übersteigen würden. Des Weiteren könnten so häufige und - offensichtlich in diesen Fällen vom Arzt als unnötig betrachtete - Wiedervorstellungen vermieden werden. 
Man hat ja nur die 10er oder 20er auf'm Kassenrezept. Und Privatrezept, dann kann ich 50 Stück aufschreiben und dann brauchen die Patienten nicht dauernd kommen. Das ist der Vorteil. Und Kosten herist es ja günstig. (I16,77)

Ein anderer Teilnehmer war der Meinung, dass auf Grund einer Vorgabe der Kassenärztlichen Vereinigung Hypnotika nicht auf Kassenrezept verordnet werden dürfen, und er u. a. deshalb routinemäßig ein Privatrezept verwenden würde (Vergleiche 5.3.7).

Wobei sowieso Schlafmittel von der KV her also gar nicht so substitutionell bezablt-bezablbar sind. Also das darf normalerweise, glaub ich, gar nicht gemacht werden. Aber das ist so ein Automatismus, dass ich eigentlich jetat nicht mehr reflektiere, sondern das immer so mache. $(\mathrm{I} 3,58)$

Ein Weiterer gab an, dass er zwar die Kostenübernahme durch die Krankenkassen in bestimmten Fällen als gerechtfertigt ansehen würde, implizierte aber durch die Verwendung des Konjunktivs, dass aktuell auch viele dieser Patienten ein Privatrezept erhalten.

Die Rolle der Krankenkasse in Bezug auf Benzodia-? Naja gut, wenn das entsprechend indiziert ist, so bei Angststörungen oder so, denk' ich schon, sollten sie's bezablen, ähm. Und meiner Meinung nach wenn jemand abhängig is' und es wird 'n Versuch unternommen, das auszuschleichen, wäre es eigentlich auch okay, wenn's auf Krankenkasse liefe, 'ne (I6, 170)

Bezüglich der Frage, wann und wie lange Benzodiazepine und Z-Substanzen von den gesetzlichen Krankenkassen bezahlt werden, scheint es an Orientierungshilfen zu mangeln insbesondere, wenn man die unter 5.5 festgehaltenen Auskünfte der KV und der Beratungsapotheke einer Krankenkasse betrachtet. Zumindest wird die dort erwähnte Anlage III der AMRL nicht als Hilfe angesehen. Diese Unsicherheit in Zusammenspiel mit dem Kontext, dass Hypnotika als abhängigkeitsinduzierende Substanzen wahrgenommen werden, deren Verwendung möglichst vermieden werden soll, bedingt die Entstehung der Idee, den Zugang zu diesen Medikamenten zu erschweren.

Zusätzlich sei angemerkt, dass bei einigen Ärzten offensichtlich der Wunsch besteht, sich mit Kollegen zu diesem Thema auszutauschen, wie das folgende Zitat zeigt.

Letzten Endes muss ich sagen, man könnte es mal im Qualitätszirkel besprechen, wie es andere manchen. Also ich weiß nicht, ob es auch als, äh, Kassenverordnung zu rechtfertigen wäre. (I2, 36)

Entsprechend dem Prinzip des theoretischen Samplings (s. 4.1.1) wurde daher die Datenerhebung ausgeweitet und die BenzoZ-Verordnung auf Privatrezept im Rahmen eines Qualitätszirkels zu diskutiert. Das Ergebnis ist unter 5.4 festgehalten. Zunächst sollen aber die weiteren Komponenten des oben abgebildeten paradigmatischen Modells der BenzoZPrivatverordnung beschrieben werden.

\subsubsection{Privatrezept nutzen, um eine Hemmschwelle zu kreieren}

Die Idee, eine Hemmschwelle zu kreieren und dadurch den Patienten den Zugang zu Benzodiazepinen und Z-Substanzen zu erschweren, hat mehrere Folgen, wie unter 5.3.8 und 
5.3.9 zu lesen ist. Jedoch wurde in den Interviews am häufigsten die erwünschte Konsequenz ,Minimierung des Konsums` erwähnt, die daher bereits an dieser Stelle und unmittelbar im Zusammenhang mit dem Hemmschwellen-Phänomen dargestellt werden soll.

Die durch das Privatrezept für den Patienten anfallenden Kosten sollen ihn dazu bringen, möglichst wenig von dem Medikament einzunehmen - indem der finanzielle Aufwand als Hürde fungiert. Somit soll eine Dosissteigerung durch Toleranzentwicklung und eine Abhängigkeit vermieden werden. Dies war der von den Teilnehmern am häufigsten genannte Grund einem kassenärztlich Versicherten ein Privatrezept zu geben.

Also prinzipiell-boshaft gesagt, es soll ein bisschen wehtun, dass sie sich dieses Schlafmittel gönnen und sie sollen nicht in Versuchung kommen das Zeugs jetzt wie Drops zu lutschen, weil sie dadurch auch gewissen Kosten haben. Das ist eigentlich so mein Hintergedanke, der dabei stebt. (I3,58)

Aber ja, aber wenn man selber bezablen muss, ist die Hemmschwelle nochmal ein bisschen größer als wenn man's dauernd auf Kasse kriegt, ne. (I4,54)

Gut, einmal, denke ich, hat das erstaunlicher Weise vielleicht auch 'nen bisschen erzieherischen Aspekt, ne, dass ich denke, wenn die Leute es selber bezablen müssen, dann nehmen sie es nicht so viel. (I10,38)

Ein Arzt nutzt das Privatrezept als Zeichen an seine Patienten, dass er die Einnahme dieser Medikamente nicht wünschenswert findet und eigentlich nicht unterstützen möchte - und unterstützte sie eben doch, indem er es ihnen ermöglichte, das Präparat zu kaufen. Ein anderer Teilnehmer gab an, so verdeutlichen zu wollen, dass Benzodiazepine und ZSubstanzen signifikante Nebenwirkungen haben, mit denen sich der Patient kritisch auseinandersetzen sollte.

Einfach auch, um, ähm, zu zeigen, ähm, es ist nicht ein Medikament wie jedes andere, wie das Mittel für Blutdruck oder Diabetes, sondern das ist was, äbm, ja, dafür muss der Patient extra noch was- zumal einfach auch, um ihn dazu zu zwingen, mehr darüber nachzudenken. Und den älteren Leuten mit den kleinen Renten-das sind echte Beträge, wenn man das wirklich regelmäßig nimmt. (I15,28)

\subsubsection{Handlungsstrategie BenzoZ-Privatrezept}

Bevor auf die intervenierenden Bedingungen und weitere Folgen des PrivatrezeptGebrauchs eingegangen wird, sollen die Dimension der Verwendungshäufigkeit sowie der von den Ärzten wahrgenommene Erfolg dieser Strategie beschrieben werden.

\subsubsection{Dimension der Handlungsstrategie BenzoZ-Privatrezept}

Wie in Abbildung $11 \mathrm{zu}$ sehen, befindet sich an beiden Enden des Dimensionsspektrums eine klare Verhaltensweise. Zwei Ärzte gaben an, keine Privatrezepte für Benzodiazepine und Z-Substanzen auszustellen; ein Teilnehmer verordnete die Substanzen „grundsätzlich“ auf Privatrezept. 
Nie

Privatrezept
In Abhängigkeit von...
Immer

Privatrezept

Abbildung 11: Die Dimension des BenzoZ-Privatrezeptes

Im mittleren Bereich zwischen diesen Extremen hängt die Entscheidung für oder gegen diese Rezeptform von verschiedenen Faktoren ab, beispielsweise vom Patiententyp, der Diagnose, der zu erwartenden Behandlungsdauer oder der Substanzklasse (Z-Substanz oder Benzodiazepin).

\subsubsection{Erfolg der Handlungsstrategie}

Fragt man nach dem Erfolg der Strategie, so ist die fast einheitliche Erfahrung der Befragten, dass noch kein Patient auf Grund der Kosten die Einnahme beenden oder zu einem Nicht-BenzoZ-Präparat wechseln wollte. Nur ein Arzt gab an, eine direkte abschreckende Wirkung beobachtet zu haben. Patientenbeschwerden über die Selbstzahlung von Schlafund Beruhigungsmitteln scheinen die Ausnahme zu sein.

Ich glaub, da gab's noch nie Ärger. Weil die Leute sind ja einfach glücklich, wenn's ibnen denn wer aufschreiben würde, ne? Und die Benzos kosten ja auch wirklich nix. (I10,79)

Häufiger beobachten die Befragten Beschwerden über die Tatsache, dass viele Erkältungsund Schmerzmittel, wie Paracetamol, nicht von den gesetzlichen Krankenkassen finanziert würden - wobei es sich hierbei ebenfalls um sehr preiswerte Medikamente handelt.

Jedoch wurde oft schon das Beibehalten einer BenzoZ-Dosis oder Einnahmefrequenz als Erfolg verbucht. Unter diesen Gesichtspunkten ist die Strategie ,Privatrezept als Hemmschwelle` möglicherweise erfolgreich bei Patienten, die entweder keine oder eine Niedrigdosisabhängigkeit haben; bei einer eskalierenden oder bereits bestehenden Hochdosisabhängigkeit ist sie es allerdings nicht, wie das nachfolgende Zitat belegt.

I: Okay, und, ähm, das's ja- nur mal so für mich, ob ich's richtig verstanden habe: Also die Idee dabinter [hinter dem Privatrezept] ist, dass das den Konsum einschränkt?

P: Ja, ja genau. (...)

I: Und, ähm, klappt das gut? Also haben Sie das Gefühl, dass die Leute so mit ibrem Konsum da sind, wo Sie sagen: Ok, das könnte ich irgendwie noch, ähm- ((wird unterbrochen))

P: Also bis auf die Abhängigen, ja.

I: Okay, und wie ist es bei den Abbängigen?

$P$ : Ja, die sind abbängig. Ne, die schmeißen sich auch zwei ein. (I4,71) 


\subsubsection{Privatrezept in Abhängigkeit vom Patienten(typ)}

Ein Faktor, der die Handlungsstrategie und somit die Entscheidung über die Rezeptform beeinflusste, war die Patientenwahrnehmung des Arztes. „Brave“ Patienten, die kleine Menge einnahmen und mit ihrem Verhalten den Erwartungen des Arztes entsprachen, erhielten eher ein Kassenrezept.

Ich sag immer, die Braven, da hab ich auch so zwei im Kopf, die ja alle halbe Jahre mal kommen und sich mal Zopiclon holen, denen könnte ich das ja im Prinæip auch auf Kasse verordnen (I4,98)

Patienten, die dies nicht taten - beispielsweise ihre Flugangst nicht anders außer mit Medikamenten behandeln lassen wollten, obwohl dies aus Sicht des Arztes indiziert wäre - erhielten eher ein Privatrezept. Ebenfalls erhielten oft Patienten im Dienst oder Vertretungsfall - also Unbekannte - oder Patienten, von deren Ehrlichkeit man nicht überzeugt war, ein Privatrezept.

Also, das ist wieder so dieses Bauchgefühl. Und reinfallen kann man. Also, prinzipiell sollte man es allen Leuten dann glauben, egal, ob man die kennt, oder nicht, ne. Das ist ja immer so eine Direktive, dass man erstmal glaubt, was der Patient eræähblt. Und wenn ich das nicht glaube, dann habe ich ja die Alternative, zu sagen „du kriegst jetz̨t ein blaues Rezept oder gar nichts." Und spätestens dann weiß man ja, was, was Sache ist. Wie ungehalten die dann reagieren, unter Umständen. (I8, 352)

Zwei Ärzte stellten für Patienten ein Kassenrezept aus, deren finanzielle Lage sie gut kannten und wussten, dass ein BenzoZ-Privatrezept nicht erschwinglich war.

\subsubsection{Privatrezept in Abhängigkeit von der Diagnose und Problemdauer}

Als weitere intervenierende Bedingungen zeigten sich die Diagnose eines Patienten und die vom Arzt vermutete Dauer des Problems. Die Ergebnisse geben diesbezüglich zwei einander entgegengesetzte Herangehensweisen wieder. Welche davon verfolgt wird, ist abhängig vom jeweils Befragten.

Einerseits wurde bei „reinen“ - also nicht durch psychiatrische oder organische Erkrankungen bedingte - Schlafstörungen oder Flugangst ein Privatrezept gewählt. Interessant ist, dass eine solche primäre Insomnie von vielen Teilnehmern als ein Problem eingestuft wurde, welches der Verschreibung von Benzodiazepinen und Z-Substanzen eigentlich gar nicht bedarf (s. 5.2.3.1). Wie das untenstehende Zitat zeigt, gab ein Arzt an, auch in einer akuten Krisensituation, wie beispielsweise einem Trauerfall ein Privatrezept auszustellen, wobei die meisten Teilnehmer sich hier einer anderen Strategie bedienten und einzelne Tabletten aus einem Praxis- oder Vorrat im Hausbesuchskoffer herausgaben (Vergleiche Abb. 8c).

Ja, generell handhaben wir das schon bei sicherlich mehr als achtzig Prozent der Patienten so, wenn das jetż nur um reine Schlafstörungsbefindlichkeiten geht, dass wir sowohl Lorazepam als auch Zopiclon, zum Beispiel auch auf Privatrezept verordnen. Zoplicon fast zu ja 90 bis 95 Prozent, würd ich denken. $(17,46)$ 
Ja, das ist auch schon wieder abhängig von der Schwere der Erkrankung, ja. Also ich würde es, ja selbst wenn es bei solchen kurzen Sachen geht, die wir vorbin auch hatten, Stichwort Trauerfall, Beerdigung oder Flugreise dann auf Privatrezept verordnen. (I7,78)

Entsprechend dieser ersten Herangehensweise wurde, ähnlich den unter 5.2.3.2 aufgeführten Diagnosen, die die Verwendung von BenzoZ generell rechtfertigten, ein Kassenrezept bei psychiatrischen und schweren organischen Erkrankungen verwendet. In einigen Fällen wurde die Bestätigung durch einen Spezialisten, wie einem Neurologen oder Psychiater, veranlasst.

Ob nun nachgewiesene Angststörung, ne oder mit psychiatrischen Diagnosen, die kriegen das von mir auf, ähm, auf Kasse. Die anderen kriegen nur Privatrezepte. $(\mathrm{I} 4,54)$

Also wenn ich eine harte Diagnose habe, und die beim Neurologen mitlaufen, und der das auch unterstütrt, dass das so läuft, es gibt ja auch bestimmte Depressionen mit Angststörungen dabei (...), da nehme ich immer einen Facharyt mit rein. Also da, äbm, wenn das auf Dauer, auf, ähm, auf Kasse laufen soll, brauche ich ja einfach auch Rückhalt, weil, sonst kriege ich gleich irgendwie Meldung von der Krankenkasse. $(\mathrm{I} 8,86)$

Das letzte Zitat spiegelt zudem die Sorge vor einer Auseinandersetzung mit der Krankenkasse und einem Regress wider; dessen Vermeidung neben der Minimierung des Konsums als Ziel oder Folge des Privatrezeptes angesehen werden kann, wie unter 5.3.9 erläutert wird. Mit Ausnahme der primären Insomnien lässt sich eine relativ klare Zuordnung der Rezeptform in Abhängigkeit von der Dauer des Problems erkennen: Flugangst und Trauerreaktionen sind eher kurzfristig. Hierfür wird das Privatrezept verwendet. Schwere organische und psychiatrische Erkrankungen stellen langfristige Probleme dar, und die Patienten erhalten ein Kassenrezept.

Anderseits verfolgten einige der Ärzte die entgegengesetzte Vorgehensweise und nutzen bei kurzfristigen Problemen, wie den oben genannten, das Kassenrezept und langfristig ein Privatrezept. In diesem Fall wurden Patienten, die ein Privatrezept erhielten, oft als geerbt betrachtet.

Ein Teilnehmer hatte diesbezüglich der Verordnungsdauer und Rezeptform seinen eigenen, klaren Algorithmus geschaffen, um mit der Ambivalenz umzugehen.

Wenn die das-, mbh, kriegen, das nur zwei Wochen, 'ne, wenn es zum Beispiel neu ist, auf Kasse. Ansonsten kriegen sie eigentlich alle so auf- auf Privatrezept. (...)

So hab ich das- so bab ich das für mich entschieden, ja. 'Ne, die kriegen dann einmal so 'ne N1-Packung auf Kasse, 'ne. (I6, 44+48)

\subsubsection{Privatrezept in Abhängigkeit von der Substanzklasse}

Ebenfalls beeinflusste die Medikamentenwahrnehmung des jeweiligen Arztes die Handlungsstrategie. Einige der befragten Ärzte schrieben vorwiegend für die ausschließlich als Schlafmittel zugelassenen Z-Substanzen ein Privatrezept aus und begründeten die vermehr- 
te Verwendung des Kassenrezeptes bei Benzodiazepinen damit, dass diese nicht nur als Schlafmittel, sondern bei psychiatrisch kranken Patienten als Beruhigungsmittel eingesetzt würden. Ein Teilnehmer verwendete jedoch für diese Indikation eine Z-Substanz und verordnete entsprechend der Herangehensweise ,psychiatrische Erkrankung: Kassenrezept Zopiclon zu Lasten der GKV, wie die beiden nachfolgenden Zitate zeigen.

Zopiclon wird auch ungefähr so bei 10, 12 Patienten bei mir verordnet, die wiederrum sind aber dann in der Regel nicht nur ausschließlich als Schlafmittel eingesetzt. Sondern die baben dann auch noch psychische Nebenprobleme, die das etwas bedingen. (I3,10)

Nein, ich gebe keine Schlafmittel (2) auf Kasse ab. (...) Es sein denn, die Z-Substanz Zopiclon, ne das, okay, das geht auf Kasse. Das sind aber dann auch wirklich Patienten, die das in der Daueranwendung haben. Wie gesagt, dass sind weniger als von den Benzodiazepinen. Die Benzodiazepine zur Schlafinduktion werden nur privat verordnet, nicht auf Kasse. Definitiv nicht. (I3, 60+62)

\subsubsection{Privatrezept und Routine - Einflussfaktor oder Folge?}

Also, die meisten Leute kennen das sowieso. Das wird eigentlich immer auf Privatrezept verordnet. (I10,77)

Da das BenzoZ-Privatrezept eine verbreitete Praxis ist, stellt sich in einigen Fällen eine Routine ein, die von Ärzten und Patienten nicht mehr hinterfragt wird; so wird beispielsweise bei Praxisübernahme die vom Vorgänger gewählte Rezeptform beibehalten. Einem Teilnehmer war das Verschreibungsverhalten der benachbarten Allgemeinärzte bekannt, so dass bei Vertretungspatienten je nach Hausarzt entweder ein Kassen- oder Privatrezept gewählt wurde.

Diese existierende Routine beeinflusst, genau wie die anderen unter 5.3.4 bis 5.3.6 dargestellten intervenierenden Bedingungen die Handlungsstrategie ,Privatrezept ausstellen' und sorgt dafür, dass diese immer weiter fortgeführt. Auf der anderen Seite entsteht durch die permanente Nutzung des Privatrezeptes irgendwann ein alltäglicher Ablauf, so dass die Routine auch als Folge der Handlungsstrategie betrachtet werden könnte. Letztendlich ist dies von der jeweiligen persönlichen Betrachtungsweise abhängig.

\subsubsection{Privatrezept zur Entlastung der Versichertengemeinschaft}

Neben der intendierten Minimierung des Konsums versprechen sich manche Ärzte durch den Aufbau einer Hemmschwelle mittels Verwendung des Privatrezeptes das Erreichen weiterer Ziele. So soll ein Medikament, das nicht medizinisch notwendig ist, nicht von der Gemeinschaft finanziert werden - auch wenn es sich bei Benzodiazepinen und ZSubstanzen um preiswerte Präparate handelt, wie im nächsten Abschnitt noch einmal aufgegriffen wird. Der in der Literatur oft verwendete Begriff der Wunschverordnung kam allerdings in keinem Interview vor.

Ja, das sind die Leute- das sind auch die 80-Jährigen, die also ibr Schlafmittel immer haben wollen unddie damit [mit dem Privatrezept] belasten halt die Versichertengemeinschaft nicht, sondern sagen gut, 
dieses ist kein- ist nicht medirinisch geboten. Schlimmstenfalls bist du mal ne Nacht wach, ne. Und, äh, wenn es aber unbedingt sein soll oder wenn die's unbedingt wollen und ich auch weiß, dass ich ibnen damit nicht schlimmeren Schaden zufüge, dann müssen sie es halt bezablen. Und das heißt, dann gibt's das auf Privatrezept, weil es ja nicht rezeptfrei ist. (I13,77)

In anderen Fällen wird der Missbrauch zwar klar erkannt und benannt; hier soll die Versichertengemeinschaft erst recht nicht dafür aufkommen.

Ja. Ja. Das- kommen ja viele im Vertretungsfall und wenn hier einer ankommt, dann kriegt er das auch so- das ist denn meisten schon aus dem Drogensektor. Dass die dann da sagen: ich brauch das, so ungefähr. Gut, das sind dann natürlich Sachen, die man dann nicht auf Kassenkosten aufschreibt. Ne? (...)

Und da sind dann-da ist der echte Missbrauch dann da. Also nicht für den Patienten, weil das sind ja häufig Drogenabhängige, die sich andere Drogen besorgen indem sie die Rezepte vertickern (I17, 33+34)

\subsubsection{Privatrezept zur Budgetentlastung und Regressvermeidung}

Eine weitere Konsequenz innerhalb des in Abbildung 10 dargestellten paradigmatischen Modells ist die Budgetentlastung und Regressvermeidung. Die Angst vor einem Regress beruht nicht immer auf eigenen Erfahrungen, sondern teilweise auf Grund von Erzählungen von oder über Kollegen. Es entsteht aber die Sorge, dass es einen selbst treffen könnte. So merkte der Arzt, der angabt, den Rückhalt eines Spezialisten zu brauchen, um eine „Meldung von der Krankenkasse“ zu vermeiden (s. 5.3.5), auch Folgendes an:

P: Da babe ich auch noch nie irgendwie einen Regress oder irgendwas drüber gekriegt, weil, wenn man da auffällig wird, dann kriegt man ja eine Meldung. ((lachend)) Also, da kann man ja gar nicht mehr gucken, so schnell, wie man dann angezählt wird, wenn da zu viel von verordnet wird. Also das ist ja auch bekannt, dass wir dann da gleich, ähm, sozusagen in Überwachung sind, und dann eine Meldung kriegen, ähm „was, was macht ibr da?" Ne, und die Substanzen sind im Vergleich viel zu hoch verordnet, und dann kommt ja gleich der Elfmeter. Und dann muss man ja eh gucken, was man macht.

I: Aber bis jetzt gab es noch keine Rückfragen?

P: Hm ((verneinend)).

I: Oder Feedback oder so, von der Krankenkasse?

P: Nein, in keinster Weise. Also, ich weiß, dass es das gibt, aber ich hatte das noch nicht. (I8,158)

Ein Teilnehmer hatte jedoch eine relativ unmittelbare Erfahrung in der eigenen Praxis gemacht und erklärte, dass dies nun nicht nur sein Verschreibungsverhalten, sondern auch die Wahl der Rezeptform beeinflussen würde.

Da gab's auch 'ne Regressforderung. Aber die kommt obne Oxazepam nicht zurecht. Also hat man's dann irgendwie weiter gemacht. Dass es immer so ging. Die Regressforderung dann begründet. Also das hat mein [mittlerweile berenteter] Kollege alles gemacht. Das ist- dafür bin ich zu kurz in der Praxis, als dass das jetzt in der kurzen Zeit auf mich zugekommen wäre. Das ist ja immer mit 'ner Verzögerung, das kommt dann wahrscheinlich nächstes Jahr oder übernächstes Jahr auf mich zu, ja. 
Jaa, ja, ja, also dieser eine Fall, ja, natürlich, das überlege ich mir dann schon, ja, wenn ich 'nem anderen das verordne, das, was auf den zutrifft, obwobl es vielleicht 'ne andere Krankenkasse ist, dann denke ich schon, könnte das möglicherweise bei dem Patienten auch so sein, dass man dann sich für seine Medikation oder sein Medikationsverbalten rechtfertigen muss. Oder es kommt'ne Regressforderung, dann überlegt man schon, wie man's macht. Und wenn es aber Patienten sind, die das, sagen wir mal, schon jabrelang nehmen, dann hat man gar keine andere $W$ abl als das zu verordnen. Und dann bliebe eigentlich nur das Privatrerept. $(15,22+116)$

Drei Ärzte gaben an, dass eine Budgetentlastung ein Grund für das Ausstellen eines BenzoZ-Rezeptes sei; wobei einer der drei im Gespräch anmerkte, dass Benzodiazepine und ZSubstanzen sehr preisgünstige Medikamente seien. Diese Meinung wurde von drei anderen Teilnehmern geteilt; einer davon erwähnte explizit, dass die Budgetersparnis seiner Meinung nach kein Grund für ein BenzoZ-Privatrezept sei.

\subsection{Das Privatrezept als Thema im Qualitätszirkel}

\subsubsection{Teilnehmer des Qualitätszirkels}

Zum Qualitätszirkel (QZ) kamen keine Männer und insgesamt sechs Frauen. Vier davon hatten im Vorfeld noch nicht an der Studie teilgenommen und waren der Autorin dieser Arbeit unbekannt. Die Moderatorin war bereits einzeln interviewt worden. Als sechste Person fungierte die Autorin sowohl in der Rolle der Beobachterin als auch als Referentin. Wie sich im Gespräch herausstellte, besaß eine Teilnehmerin den Facharzt für Neurologie und war zu Zeit als Ärztin in Weiterbildung in einer allgemeinmedizinischen Praxis angestellt. Alle anderen hatten eine allgemeinmedizinische Facharztqualifikation. Die Dauer der Diskussion betrug 85 Minuten.

\subsubsection{Inhalte der QZ-Diskussion}

Die Aussagen der QZ-Teilnehmerinnen stimmten in vielen Punkten mit denen der Einzelinterviewteilnehmer überein. Es wurde festgestellt, dass es früher insgesamt mehr Dauerverordnungen gab, entsprechend dem Vorordnungskontext (s. 5.2.2 Verordnungskontext) des übergeordneten paradigmatischen Modells (s. Abb. 3). Akute Schlafstörungen seien aber nach wie vor sehr häufig und Alternativen zu Benzodiazepinen und ZSubstanzen oft nicht wirksam. Somit empfanden auch die QZ-Teilnehmerinnen eine Ambivalenz gegenüber diesen Medikamenten (Vergleiche 5.2.4 Ambivalenz als zentrales Phänomen). Nichtsdestotrotz würden zunächst schlafhygienische Maßnahmen angesprochen werden; eine Teilnehmerin hatte gute Erfahrungen mit einem Schlaftagebuch gemacht, da die Patienten so wüssten, dass ihr Problem ernst genommen würde. Das auch als Schlafmittel verwendete Antidepressivum Mirtazapin wurde bezüglich der Nebenwirkungen unterschiedlich eingeschätzt; eine der Anwesenden hatte einen deutlichen Überhang und Tagesmüdigkeit bei ihren Patienten beobachtet. Dies deckt sich im Wesentlichen mit der 
Handlungsstrategie „Nutzung der Alternativen“ der Einzelinterviewteilnehmer (Vergleiche 5.2.5.1 Nutzung der Alternativen). Eine Teilnehmerin betonte, dass sie die kurzfristige BenzoZ-Verordnung in Stresssituationen, wie z. B. während eines Krankenhausaufenthaltes angemessen fände (Vergleiche 5.2.3.2 Ursachen der Verwendung von BenzoZ). Jedoch gab es unterschiedliche Meinungen, ab wann eine Abhängigkeit entsteht.

Tn1: (...) Und viele wollen auch nur zebn Tabletten haben und ich meine, da ist dann wirklich kein großes Problem mit verbunden. Und wenn ich überlege, eigentlich kommen dann ganz selten dieselben noch mal. Also scheint das dann auch in so'm kurzen Rabmen zu reichen, würde ich denken. Also wenn das so'ne akute Belastungssituation ist.

Tn2: Also bättest du keine Angst damit ne Abhängigkeit-

Tn1: Bitte?

Tn2: Du bättest keine Angst, in so kurzer Zeit diese Gewohnheit-

Tn1: Nö, das wirst du ja nicht.

Tn2: Naja, du wirst nicht körperlich abhängig ((wird unterbrochen))

Tn(w): Man sagt vier Wochen, also so habe ich es immer gelesen. Vier Wochen am Stück, dann ja. Das sage ich dann auch. (QZ, 203)

Bezüglich der Verordnungsform gab eine Teilnehmerin Folgendes an:

Tn3: Also die ersten drei Wochen darf man ja auf Kassenrezept, ne? Dann ist es ja, wenn jemand akut nen Schlafmittel nimmt, darf man ja drei Wochen Kassenrezept und dann finde ich das auch gut, dass es noch mal ne Überlegung ist, ob man es nicht vielleicht versucht, sich abzugewöhnen, weil man dann eben das selber zahlen muss. (QZ, 104)

Diese Aussage wurde von den anderen nicht unmittelbar kommentiert - lediglich fügte eine andere Teilnehmerin hinzu, dass bei bekannter Abhängigkeit auch länger verordnet werden darf. Interessant ist, dass die Nutzung des BenzoZ-Privatrezeptes zur Reduktion der Einnahmemenge zwar im obigen Zitat erwähnt, aber ansonsten nicht weiter thematisiert wurde - im Gegensatz zu den Einzelinterviews, in denen sich das Kreieren einer Hemmschwelle als zentrales Phänomen eines untergeordneten paradigmatischen Models zeigte (s. Abb. 10), auf das mit der Strategie „Ausstellen eines Privatrezeptes“ reagiert wurde. Allerdings wurde auch in der QZ-Diskussion deutlich, dass Unklarheiten darüber bestehen, wann und wie lange Schlaf- und Beruhigungsmittel auf Kassenrezept verschrieben werden dürfen (Vergleiche 5.3.1 Privatrezept auf Grund von Unsicherheit bezüglich bestehender Richtlinien) und wie mit Patienten umzugehen ist, die diese Medikamente schon lange nehmen.

Tn4: Aber ich finde, es ist nen schwieriges Thema. Weil wir in der Praxis-also ich habe zwei, an die ich mich erinnere, ähm, wo es wirklich so ist, dass die ungefähr seit dreißig Jahren das nehmen. Und das kann man hundert Mal besprechen, dass man es anders machen will. Versucht es mit anderen Sachen. Anderseits sind da auch Psychiater mit im Team, der ihm [dem Patienten] immer wieder was Anderes auf- 
schreibt. Und dann kommt er [der Patient] zu mir und ist völlig vervvirrt, weil er dann alles zusammen genommen hat, also zwei, drei Sachen. Also da machen wir es in der Regel so, weil wir auch nicht mebr wissen, dürfen wir jetzt oder dürfen wir nicht, dass es tatsächlich auf nem Privatrezept geht. (QZ, 116)

In der Regel erhielten jedoch Patienten mit psychiatrischen Erkrankungen, wie beispielsweise einer Angststörung, ein Kassenrezept - so wie dies auch als intervenierende Bedingung in den Einzelinterviews beobachtet worden war (Vergleiche 5.3.5 Privatrezept in Abhängigkeit von der Diagnose und Problemdauer). Und auch in der QZ-Diskussion fand sich das Konzept der medizinisch nicht notwendigen Verordnungen von Schlaf- und Beruhigungsmitteln; diese wurden als „Lifestyle Medikamente“ bezeichnet und privat rezeptiert. Unsicherheit bestand bzgl. der Verschreibung dieser Medikamente für die „Reiseapotheke“, da die Kosten für Reiseimpfungen nicht oder nur teilweise von den gesetzlichen Krankenkassen getragen werden und Patienten hierfür ein Privatrezept erhalten. Zählt man jedoch Flugangst, genau wie andere Angststörungen, zu den psychiatrischen Erkrankungen, müsste ein Kassenrezept ausgestellt werden.

Im Gegensatz zu den Einzelinterviews wurde die Frage aufgeworfen, ob Patienten mit einer iatrogenen Abhängigkeit für die Behandlungsfehler der Ärzte bestraft werden sollten, indem sie nun selbst für die Medikamente bezahlten. Auf der anderen Seite wurde die Mitwirkungspflicht des Patienten diskutiert, der bei bekanntem Abusus einen Entzug nicht einfach ablehnen und erwarten könnte, dass die gleiche Menge weiter verschrieben wird. Allerdings finden sich, wie von einer der Anwesenden bemerkt, in der ärztlichen Arbeit diverse Beispiele, in denen der Patient trotz kontinuierlich gesundheitsgefährdendem Verhalten weiter behandelt wird, z. B. Diabetiker mit Adipositas oder Raucher mit Bronchialkarzinom. Hieraus ergibt sich eine gewisse Machtlosigkeit.

Tn5: Ich kann sie ja nicht zwingen, äbm ibre Abhängigkeit behandeln zu lassen. Gleichzeitig trage ich das natürlich auf ne Art mit, wie bei Opiatabbängigen oder Drogenabhängigen. (QZ, 426)

Zustimmung fand die Aussage, dass ein stationärer Entzug nicht immer das Beste für alte Patienten sei und Krankenhausaufenthalte vermieden werden sollten, da zusätzlich bestehende und bis dato gut kontrollierte Erkrankungen sich hierdurch verschlimmern könnten.

\subsection{Weitere Ergebnisse}

\subsubsection{Das Privatrezept aus der Laien-/Bürgerperspektive}

Wie unter 4.6 erwähnt, wurden die Daten dieser Arbeit im Rahmen der methodischen Qualitätssicherung während des Doktorandenkolloquiums des Instituts für Sportwissenschaften der Universität Göttingen vorgestellt und diskutiert. Dies diente dazu, das Prinzip der Offenheit zu wahren und ggf. vorhandene berufsspezifische Vorannahmen der Auswertenden zu minimieren. Im Rahmen des Kolloquiums kamen circa zehn Sportstudenten zusammen, bei denen es sich um junge und wahrscheinlich vorwiegend gesunde Menschen 
gehandelt haben dürfte, ohne das persönlich Daten der Teilnehmer abgefragt wurden. Als medizinische Laien nahmen die Studenten nicht die Perspektive des Arztes, sondern die des Patienten ein. Die Strategie, durch das Ausstellen eines Privatrezeptes auf die Gefahren von Benzodiazepinen und Z-Substanzen hinzuweisen - denn sie seien kein „Medikament wie jedes andere“ - und die eingenommene Menge zu reduzieren, wurde als nicht erfolgsversprechend bewertet. Im Gegenteil, da auch pflanzliche Präparate und beispielweise Erkältungsmedikamente komplett selbst bezahlt werden müssen, bedeutete für die Studenten ein Privatrezept eine Zuordnung der Hypnotika zu den oben genannten Mitteln; sie empfanden Medikamente, die nicht von der Krankenkasse bezahlt wurden als eher nebenwirkungsarm. Der Unterschied zwischen einem grünen und einem blauen Rezept schien den meisten Anwesenden nicht klar zu sein, zumal in beiden Fällen das Präparat eigenständig finanziert werden muss. Folglich wäre es auch kein Problem etwas mehr von dem vermeintlich harmlosen Medikament zu nehmen. Auf der anderen Seite bedeutete in ,richtiges“ Rezept, dass man sich als Patient an die Anweisungen des Arztes zu halten hat.

Die Sportstudenten sahen zudem eine Parallele zum Doping - insbesondere hervorgerufen durch folgendes Zitat:

Ganz schwierig. Ja, ja klar, 'ne. Die-das sind aber auch Leute, die definitiv auch sagen: ,Okay, wenn Sie's nicht aufschreiben, dann geb' ich auf den Markt. 'Das- das is äb-((lachend)) kenn ich auch genug. Dann sagt man auch, komm, dann lieber so 'n bisschen kontrolliert, als dass du da irgendwelche Strukturen stärken oder so. Und eben anbieten, dass da'n Halt haben. (I6,126)

Sie verwiesen auf einen Text von Singler und Treutlein, der sich mit den historischen und soziologischen Aspekten des Dopings im Spitzensport befasst und im Sammelwerk Olympia als Bildungsidee veröffentlicht wurde (Singler und Treutlein 2013, Seite 161). Hier heißt es u. a., dass „Anabolika lange Zeit für ungefährlich gehalten“ wurden und dass, „der Sportarzt ständig mit entsprechenden Wünschen von den Athleten angegangen“ wird (Steinbach 1968, Seite 486). Des Weiteren wird darauf hingewiesen, dass obwohl 1976 Anabolika schon international als Dopingmittel gelistet waren, sich viele deutsche Sportmediziner zu der Zeit für die Verwendung unter Aufsicht aussprachen: „Wenn Ärzte ,Nein“ sagen, dann gibt es überhaupt keine Kontrolle. Es ist deshalb doch besser, mitzugehen, zu steuern und sinnvoll zu helfen als zu sagen: ,Wir sind völlig dagegen!“" (Singler und Treutlein 2013, Seite 163). Das Zitat stammt von Dirk Clasing, dem damaligen Sprecher der Arbeitsgemeinschaft der Verbandsärzte, der in Bezug auf Doping unter ärztlicher Aufsicht auch den Begriff der „praktischen Toleranz“ prägte. Singler und Treutlein griffen dies auf und kamen zu folgender Aussage: „Um Überdosierungen zu vermeiden, sahen Ärzte sich wohl nicht selten als Berater; ihre Rolle des Behüters war dann aber von der einer treibenden Kraft nicht mehr zu unterscheiden.“ (Singler und Treutlein 2013, Seite 164) 


\subsubsection{Auskunft von Kassenärztlichen Vereinigungen}

Ein Teilnehmer der Studie berichtete, sein Vorgänger habe eine lange bestehende Schlafstörung nicht als ,chronische Schlafstörung' bezeichnet und daher eine Regressforderung bezüglich der verwendeten Hypnotika erhalten. Laut telefonischer Auskunft der KV Niedersachsen (KVN) gibt es aber keine einheitliche Liste mit Diagnosen, deren Verwendung eine garantierte Kostenübernahme durch die Krankenkasse bedeuten würde. Dies sei immer eine Einzelfallentscheidung und würde evtl. von unterschiedlichen Kassen unterschiedlich bewertet werden. Jedoch sollten die Kosten von den Krankenkassen übernommen werden, wenn der Arzt die Verordnung für medizinisch notwendig hält - ohne dass dies näher definiert ist. „Wunschverordnungen“ sind laut Auskunft der KVN privat zu rezeptieren.

In Streitfällen mit der Krankenkasse könnte sich der Arzt- insbesondere wenn es um eine Verordnung von unter 4 Wochen geht - auf die Anlage III der AMRL berufen. Will der Arzt aber Streitfälle vermeiden und auf der „sicheren Seite“ sein, so könnte er ein Privatrezept ausstellen und seinem Patienten raten, dieses bei der entsprechenden Krankenkasse einzureichen. Ein Recht auf eine kassenärztliche Verordnung hätten Patienten bzgl. Benzodiazepinen und Z-Substanzen nicht - selbst wenn es sich um einen medizinisch gut begründeten Fall handelt.

Die Kassenärztliche Vereinigung Bayern (KVB) bietet eine telefonische Beratung nur für in Bayern niedergelassene Ärzte an und bat um eine schriftliche Anfrage per E-Mail, die jedoch bereits einen Tag später durch eine - laut Signatur - Apothekerin folgendermaßen beantwortet wurde:

„Seit der Neufassung der bundesweiten Arzneimittelrichtlinie durch den G-BA [Gemeinsamen Bundesausschuss] 2009 dürfen auf Kassenrezept die genannten Substanzen [Benzodiazepine und Z-Substanzen] nur noch für maximal 4 Wochen und darüber hinaus gehend nur in streng begrenzten medizinischen Einzelfällen verordnet werden. Entsprechend hat sich das Verordnungsverhalten der Ärzte verändert, was wir auch an unseren diesbezüglichen Arzneimittelanalysen nachweisen konnten. Dass leider eine Dunkelziffer von Ärzten auf die Verordnung auf Privatrezept ausweichen, ist ein offenes Geheimnis, kann und darf aber nicht freimütig akzeptiert werden. Aus unserer Sicht darf die kontroverse Diskussion mit den Patienten und im Ergebnis aus medizinischer Sicht die notwendige Verweigerung von Benzodiazepin- und Z-Substanzen von Ärzten nicht gescheut werden.

Aus bayerischer Sicht könnten Ärzte im Rahmen von Einzelfallrückforderungen von Krankenkassen auffällig werden, wenn sie ungewöhnlich hohe Mengen der genannten Substanzen trotz Verordnungseinschränkung verordnen. Dies dürfte jedoch für das gesamte Bundesgebiet gelten. Im Rahmen einer statistischen Auffälligkeitsprüfung dürften Benzodiazepine und Z-Substanzen weder im Rahmen einer herkömmlichen Richtgrößen- bzw. Durchschnittsprüfung, wie sie seit Ende 2014 in Bayern nicht mehr gilt, noch im Rahmen 
der Bayern-spezifischen Wirkstoffvereinbarung zu Regressanträgen der Krankenkassen führen."

\subsubsection{Auskunft von Krankenkassen}

Bittet man die AOK um telefonische Auskunft zum Thema Hypnotika auf Privatrezept, wird man mit der Beratungsapotheke verbunden. Ein Apotheker erläutert zunächst, dass es entscheidend sei, für welche Indikation ein Medikament zugelassen ist. Für Z-Substanzen und Benzodiazepine, die eine ausschließliche Zulassung als Schlafmittel (Hypnotikum) haben, gilt die Anlage III der AMRL „ohne Wenn und Aber; das heißt nach vier Wochen ist Schluss“. Dabei hat ein Kassenpatient einen Anspruch auf ein Kassenrezept - so es sich um einen In-Label Use handelt, also sich der Verschreibungsgrund mit der entsprechenden Zulassung deckt. Hierzu wird von der AOK auf den Bundesmantelvertrag - Ärzte verwiesen. Dieser Bundesmantelvertrag für Ärzte ist zwischen der Kassenärztlichen Vereinigung und dem GKV-Spitzenverband geschlossen worden und besagt u. a. Folgendes:

\18 Zuzahlungspflichten der Versicherten und Vergütungsanspruch gegen Versicherte, Absatz 8:

„Der Versicherte hat Anspruch auf Sachleistung, wenn er nicht Kostenerstattung gewählt hat. Vertragsärzte, die Versicherte zur Inanspruchnahme einer privatärztlichen Versorgung an Stelle der ihnen zustehenden Leistungen der gesetzlichen Krankenversicherung beeinflussen, verstoßen gegen ihre vertragsärztlichen Pflichten.

Der Vertragsarzt darf von einem Versicherten eine Vergütung nur fordern,

1. wenn die elektronische Gesundheitskarte vor der ersten Inanspruchnahme im Quartal nicht vorgelegt worden ist bzw. ein Anspruchsnachweis gemäß \19 Abs. 2 nicht vorliegt und nicht innerhalb einer Frist von zehn Tagen nach der ersten Inanspruchnahme nachgereicht wird,

2. wenn und soweit der Versicherte vor Beginn der Behandlung ausdrücklich verlangt, auf eigene Kosten behandelt zu werden, und dieses dem Vertragsarzt schriftlich bestätigt,

3. wenn für Leistungen, die nicht Bestandteil der vertragsärztlichen Versorgung sind, vorher die schriftliche Zustimmung des Versicherten eingeholt und dieser auf die Pflicht zur Übernahme der Kosten hingewiesen wurde.“

Des Weiteren findet sich folgender Hinweis:

$\int 25$ a Verordnung von veranlassten Leistungen, Absatz 5/6:

„Wird die Verordnung vom Patienten als Privatbehandlung gemäß \18 Abs. 8 Nr. 2 gewünscht, ist dafür ein Privatrezept zu benutzen. Die Verwendung des Vertragsarztstempels auf diesem Privatrezept ist nicht zulässig. 
Verlangt ein in der gesetzlichen Krankenversicherung Versicherter die Verordnung von veranlassten Leistungen, die aus der Leistungspflicht der gesetzlichen Krankenversicherung ausgeschlossen oder für die Behandlung nicht notwendig sind, kann die Verordnung nur auf einem Privatrezept vorgenommen werden. Die Verwendung des Vertragsarztstempels auf diesem Privatrezept ist nicht zulässig.“ (Kassenärztliche Bundesvereinigung 2017)

Somit stimmen die Aussagen der KVN und der AOK nicht überein. Des Weiteren verweist die Beratungsapotheke der AOK daraufhin, dass bei einer längeren Verordnung von Schlafmitteln über vier Wochen hinaus der Arzt mit einer Prüfung seitens der Versicherung rechnen und durch seine Dokumentation beweisen muss, dass die Weiterverordnung trotzdem noch indiziert ist. Nichtsdestotrotz würde dem nur in 1\% der Fälle stattgegeben; folglich droht zu 99\% eine Regressforderung. Anders verhält es sich mit Benzodiazepinen, die eine weitere oder andere Zulassung haben, z. B. als Beruhigungsmittel (Sedativum). Diese seien von der Anlage III ausgenommen und dürfen über vier Wochen hinaus verordnet werden.

Offensichtlich schwierig ist der Umgang mit Patienten, die schon seit Jahren ein Schlafmittel nehmen und bei denen eine lebenslange Weiterverordnung die einzige Option für Arzt und Patient zu sein scheint. Davon diesen Langzeitgebrauch ehrlicherweise als Arzneimittelabhängigkeit zu kodieren, rät die $\mathrm{AOK}$ ab, da eine Aufrechterhaltung der Sucht durch den Arzt juristisch bedenklich sei; jedoch würde man sich als Verschreibender dieser Verantwortung nicht automatisch entziehen, wenn man Schlafmittel auf Privatrezept verordnet. „Von daher ist die Lösung über das Privatrezept auch keine gute. Wenn jetzt der Enkel Jura studiert und zu Hause die Privatrezepte findet, es das ebenso schwierig." Auf der anderen Seite hält der Auskunft Gebende einen BenzoZ-Entzug im hausärztlichen Setting für nicht realisierbar. „Das ist so schwierig. Das können Sie im ambulanten Bereich vergessen."

Eine konkrete Aussauge bezüglich der korrekten Verordnungsweise im Fall einer bestehenden BenzoZ-Abhängigkeit ist am Telefon nicht zu erhalten, jedoch versendete der Mitarbeiter der Beratungsapotheke im Anschluss freundlicherweise folgende drei Artikel sowie den Link zu der bereits bekannten Anlage III der AMRL:

1. Vorordnung von Benzodiazepinen / Nicht ohne Indikation (Hamburger Ärzteblatt, Juni 2009)

2. Benzodiazepine, Zopiclon und Zolpidem - abwenden und absetzten (Publikation der KV Nordrhein, April 2013)

3. Verordnung von Benzodiazepinen und deren Analoga (Publikation der Hamburger Apothekerkammer, Februar 2011)

Letztere ist eine gemeinsame Handlungsempfehlung der Hamburger Ärztekammer und kassenärztlichen Vereinigung sowie der Apothekerkammer, und möchte als „Hilfestellung“ dienen. Dort wird klar festgestellt: „Besteht bei gesetzlich Versicherten eine Indikation zur Gabe der Medikamente, ist ein Ausweichen auf Privatrezept nicht zulässig." Liegen schwere psychiatrische Erkrankungen vor, die eine langfristige Verordnung von Benzodiazepinen 
über vier Wochen hinaus erforderlich machen, wird das „Einholen einer zweiten Meinung eines in der Suchtmedizin erfahrenen Arztes oder eines Psychiaters“ empfohlen. Durch eine adäquate Aufklärung des Patienten sowie die Verwendung alternativer Therapien sollen Langzeitverordnungen vermieden werden. Grundsätzlich sei bei Erkennen einer Abhängigkeit ein Entzug einzuleiten. Hierzu finden sich auf der einen Seite Aussagen wie, „dass bereits eine kurze Ansprache eines schädigenden Medikamentenkonsums beim Patienten positive Veränderungen bewirken kann", auf der anderen Seite wird zur Konsultation der oben genannten Fachrichtungen geraten. Abschließend findet sich der Hinweis, dass „,bei Verdacht auf eine nicht indikationsgerechte Verschreibung von Benzodiazepinen und Benzodiazepin-Analoga (...)der Arzt zunächst angehört [werden soll]. Sollte sich der Verdacht bestätigen, erfolgt ein abgestuftes Vorgehen, das die Elemente Beratung, Fortbildung und die Einleitung weiterer Schritte bei anhaltender Verschreibung trotz erkennbarem Missbrauchs umfasst"(Apothekenkammer Hamburg 2011). Die Publikation der KV Nordrhein stellt ebenfalls fest, dass ,bei medizinischer Indikation GKV-Versicherte Anspruch auf eine Versorgung mit Arzneimitteln haben“" und ein Ausweichen auf ein Privatrezept weder zulässig ist, noch den „,verordnenden Arzt von der berufshaftungsrechtlichen Verantwortung“" entbindet (Kassenärztliche Vereinigung Nordrhein 2013). Im Folgenden finden sich Hinweise zum ärztlichen Verhalten im Notdienst (BenzoZVerschreibungen möglichst vermeiden, geringe Dosierung, kleinstmögliche Verpackung, Hinweis an den Hausarzt geben) sowie auf den Lippstädter Benzo-Check (Holzbach 2018), um das Absetzten im Patientengespräch zu thematisieren. Zum Ausschleichen sollten Patienten auf ein Benzodiazepin mit mittlere Halbwertzeit, wie Oxazepam, umgestellt werden. Es finden sich einige weiterführende Internetadressen zu diesem Thema, aber ein direkter Hinweis zum Umgang mit Langzeitkonsumenten, für die ein Entzug nicht in Frage kommt, findet sich nicht. Der zugesendete Artikel des Hamburger Ärzteblattes ist zweigeteilt. Der erste Teil informiert über die unterschiedlichen Phasen des Langzeiteinnahme und unterteilt Patienten auf Grund ihres Konsummusters in drei Gruppen: relativ junge Menschen bis circa 50 Jahre, die die positive Wirkung der Medikamente zur Stressbewältigung nutzen, Menschen zwischen 45 und 65 Jahren, die auf Grund eines negativen Ereignisses ängstlich und depressiv reagieren, dies medikamentös kompensieren ohne die Ursache beispielsweise im Rahmen einer Gesprächstherapie behandeln zu lassen, und Menschen über 65, die schon seit vielen Jahren Benzodiazepine verordnet bekommen. Auch für diese Gruppe wird ein Entzug empfohlen, wenn auch anerkannt wird, dass „hier der Widerstand gegen das Ausschleichen des Medikamentes so groß sein kann, dass ein Entzugsbehandlung nur nach längerer Vorbereitung und mit größter Vorsicht durchgeführt werden kann (Haasen und Holzbach 2009). Der zweite Teil befasst sich mit der Verordnung von Benzodiazepinen aus berufsrechtlicher Sicht. Hierzu heißt es: „Die Unterhaltung einer Sucht mit dem Suchtstoff stellt bei der Benzodiazepinabhängigkeit grundsätzlich kein anerkanntes Behandlungskonzept dar. (...) Bei einer festgestellten Anhängigkeit wird vom Arzt ein Behandlungskonzept mit Entzugs- und Reduktionsplan und Kontrollen erwartet.“ Anderen- 
falls kann es zu Sanktionen, wie einer Rüge, einem Verweis oder einer Geldbuße kommen. Auch hier wird zu einer Überweisung zu einem „,erfahrenen Kollegen oder einer Suchtambulanz" geraten (Kiehn 2009).

Wählt man die telefonische Auskunft der BARMER Ersatzkasse und bittet um eine Beratung bezüglich der Verordnung von Benzodiazepinen und Z-Substanzen, wird man nicht an eine Beratungsapotheke weiter verbunden, sondern zu allererst daraufhin gewiesen, dass „Auskünfte am Telefon immer schwierig“ sind. Konkret auf die Privatverordnung dieser Präparate für Kassenpatienten angesprochen, heißt es dann: „Wir können immer nur auf die Arzneimittelrichtlinie und die Therapiehoheit der Ärzte verweisen. Wir dürfen den Ärzten keine Vorgaben machen. Wenn dort steht, dass das Medikament verordnungsfähig ist, dann kann es auf Kassenrezept verordnet werden; und wenn es ausgeschlossen ist, dann geht das nicht.“

Daraufhin berichtete Autorin kurze von den Ergebnissen dieser Arbeit, insbesondere, dass sich viele Ärzte unsicher sind, was wie lange bezahlt wird. Als Antwort erfährt man von der BARMER, dass sich die Betroffenen an die KV wenden müssen und wird nochmals auf die AMRL hingewiesen. Die Autorin gab zu bedenken, dass dort steht, dass BenzoZ nicht länger als vier Wochen verordnet werden dürfen, dass aber viele Patienten diese Medikamente seit Jahren nehmen - und dass sich hieraus oft die Sorge vor einer Regressforderung ergibt. „Dann kann der Arzt einen Antrag stellen, dass hier eine Ausnahme gemacht werden soll. Wir werden das dann vom MDK [Medizinscher Dienst der Krankenkassen] prüfen lassen.“ Letztendlich sei das auch eine Frage der Alternative: „Bei Herzmedikamenten ist das auch so, dass man dem Patienten dann sagen muss: da gibt es was Neues und das Alte kann nicht mehr verordnet werden. Und wenn der Patient dann darauf besteht, weil er das immer gut vertragen hat, dann ist das eine Wunschleistung - und die sind privat zu rezeptieren. Dann bleibt nur noch das Privatrezept. (...) Der Arzt muss entscheiden, ob er das vertreten kann oder nicht."

\subsubsection{Auskunft von Apotheke und Apothekenkammer}

Laut Auskunft der Apothekenkammer Niedersachsen (im Dezember 2017) regelt die Apothekenbetriebsordnung die Abgabe von Teilmengen einer Packung, z. B. zwei Tabletten. Dort heißt es: „Verschreibungen von Personen, die zur Ausübung der Heilkunde, Zahnheilkunde oder Tierheilkunde berechtigt sind, sind in einer der Verschreibung angemessenen Zeit auszuführen“ (Bundesministerium der Justiz und für Verbraucherschutz 2017) Die Apothekenbetriebsordnung verweist wiederum auf das Fünfte Sozialgesetzbuch zur Arzneimittelversorgung. Hierzu existiert ein Rahmenvertrag zwischen dem Spitzenverband Bund der Krankenkassen und dem Deutschem Apothekerverband, indem konkret Folgendes festgehalten ist:

$\int 6$ Abgabe wirtschaftlicher Einzelmengen, Absatz 2: 
„Die Abgabe einer Teilmenge aus einer Fertigarzneimittelpackung (Auseinzelung), ist nur auf ausdrückliche ärztliche Anordnung der Auseinzelung zulässig, soweit zwischen den Partnern des Rahmenvertrages [Spitzenverband Bund der Krankenkassen und Deutscher Apothekerverband] nichts Anderes vereinbart ist. Hat der Vertragsarzt eine Auseinzelung zur patientenindividuellen Versorgung (z. B. in Form einer „Verblisterung“) verordnet, bedarf es einer Einigung zwischen der Krankenkasse und der Apotheke oder deren Verbände über den Preis; $\int 3$ Absatz 2 des Rahmenvertrages gilt entsprechend.

Dort findet sich dann unter $₫ 3$ Zahlungs- und Lieferanspruch folgende Information:

„Die Höhe der Vergütung ergibt sich aus den gesetzlichen und vertraglichen Bestimmungen. Ist ein Preis auf dieser Grundlage nicht bestimmt, so bedarf es zur Entstehung des Vergütungsanspruchs nach Absatz 1 Satz 1 einer Einigung zwischen Apotheke und Krankenkasse. Das Nähere kann in den ergänzenden Verträgen nach 129 Absatz 5 SGB V geregelt werden." (Spitzenverband Bund der Krankenkassen und Deutscher Apothekerverband 2016)

Dies deckt sich mit der telefonischen Auskunft der Apothekenkammer Niedersachen, dass bei Auseinzelung nicht mehr die Arzneimittelpreisverordnung gilt und der Apotheker eigenständig einen Preis für den Mehraufwand (Um- bzw. Neuverpacken, Beilegen einer Arzneimittelinformation) berechnen darf. Ist der Patient bereit, diesen Preis zu bezahlen, ist dieses Vorgehen bei Privatverordnungen unproblematisch. Jedoch wiederspricht es möglicherweise den Lieferverträgen mit den gesetzlichen Krankenkassen, so dass das ein Einlösen eines Kassenrezeptes eventuell nicht machbar ist. Die Aussage einer Göttinger Apotheke (im Dezember 2017), es sei in diesem Fall erlaubt ohne Rücksprache mit dem Arzt auf die kleinste Packung ,aufzustocken“, konnte von der Apothekenkammer nicht bestätigt werden. 


\section{Diskussion}

Dass Benzodiazepine und die als Z-Substanzen bezeichneten BenzodiazepinRezeptoragonisten zu Nebenwirkungen wie Abhängigkeit und Entzugserscheinungen führen können, ist in den letzten Jahrzehnten immer deutlich in das Bewusstsein der Ärzte, aber auch der Patienten gerückt. Dementsprechend werden in Deutschland immer weniger dieser Schlaf- und Beruhigungsmittel verordnet. Vergleicht man jedoch die Daten der gesetzlichen Krankenkassen mit den Einkaufsstatistiken der Apotheken, stellt man fest, dass die Zahlen nicht in gleichem Maße rückläufig sind. Da der Anteil an Privatversicherten konstant geblieben ist, erhalten folglich viele gesetzlich versicherte Patienten Privatrezepte für Benzodiazepine oder Z-Substanzen. Möglicherweise erfolgt so jede zweite Verordnung dieser Medikamente. Genaue Angaben sind diesbezüglich schwierig, da Privatrezepte, die an Kassenpatienten abgegeben werden, in keiner Statistik erfasst werden und nur - durch beispielsweise einen Abgleich der oben genannten Datensätze - geschätzt werden können. Bekannt ist jedoch, dass die Gruppe der Allgemeinmediziner und hausärztlich tätigen Internisten die meisten Schlaf- und Beruhigungsmittel verordnet. Ziel dieser Arbeit war es zu erforschen, warum Hausärzte häufig das Privatrezept wählen, wenn sie diese Präparate für Kassenpatienten verschreiben, und welche Faktoren den Entscheidungsprozess beeinflussen.

Zunächst lässt sich feststellen, dass Ärzte Benzodiazepine und Z-Substanzen ambivalent wahrnehmen. Auf der einen Seite werden diese Medikamente als hilfreich und wirkungsvoll betrachtet; auf der anderen Seite sollen sie auf Grund der Nebenwirkungen möglichst nicht verschrieben werden. Das Ausstellen eines Privatrezeptes ist somit eine Handlungsstrategie, um auf das Phänomen der Ambivalenz zu reagieren, und erlaubt es, scheinbar beiden Seiten gerecht zu werden. Dem liegt die Annahme zugrunde, dass Patienten mit einem Medikament sparsamer umgehen, wenn sie einen höheren Eigenanteil bezahlen müssen. Deshalb nutzen viele Ärzte diese Rezeptform als Instrument, um den Konsum von Benzodiazepinen und Z-Substanzen einzuschränken; wobei dies oft nur bedeutet, die Verordnungsmenge und -frequenz stabil zu halten und nicht etwa das Absetzen oder Ausschleichen zu erreichen. Des Weiteren verwendet ein Teil der Ärzte das Privatrezept aus Unwissenheit oder Unsicherheit darüber, welches Medikament wie lange auf Kassenrezept verordnet werden darf. Viele Hausärzte haben im Laufe ihrer Karriere einen eigenen Algorithmus entwickelt und wählen abhängig vom Patiententyp, von der Substanzgruppe (Benzodiazepine oder Z-Substanzen), der erwarteten Problemdauer oder Diagnose eine bestimmte Rezeptform. So erhalten Patienten mit psychiatrischen Erkrankungen, wie beispielweise einer Angststörung eher ein Kassenrezept als Patienten mit primärer Insomnie. In einigen Fällen hat sich so eine Routine etabliert, die nicht mehr hinterfragt wird - insbe- 
sondere dann nicht, wenn die Handlungsstrategie ,Ausstellen eines Privatrezeptes' als erfolgreich wahrgenommen wird. Neben der bereits angesprochenen Einschränkung des Konsums versprechen sich die Ärzte davon auch die Vermeidung eines Regresses. Obwohl Benzodiazepine und Z-Substanzen preiswerte Medikamente sind, und daher eine Budgetentlastung oder eine Entlastung der Versichertengemeinschaft durch die Verwendung von Privatrezepten eine untergeordnete Rolle spielen, besteht bei vielen Ärzten die Sorge vor einer Regressforderung.

\subsection{Stärken und Schwächen der Untersuchung}

Wie in der Einleitung aufgezeigt, gibt es bereits einige Interviewstudien, die generell die hausärztliche Wahrnehmung und Verschreibung von Benzodiazepinen und Z-Substanzen darstellen (Cheung et al. 2014; Cook et al. 2007; Rogers et al. 2007). Jedoch ist die vorliegende Arbeit die erste Interviewstudie, die sich speziell den BenzoZ-Privatverordnungen für Kassenpatienten widmet. Bisher existierte in Deutschland hierzu lediglich eine Fragebogen-Studie (Hoffmann et al. 2014), die methodenbedingt die Antwortmöglichkeiten der Teilnehmer einschränkt. International finden sich keine direkt vergleichbaren Arbeiten, da das Thema unmittelbar an das deutsche Krankenkassensystem geknüpft ist. Durch die Auswertung der teilstrukturierten Interviews lassen sich die Motive der Hausärzte und die einflussnehmenden Faktoren wesentlich detaillierter abbilden und verstehen, und es konnte eine Theorie dazu generiert werden. Auch Aspekte, die im Vorfeld nicht von den Forschenden antizipiert worden, konnten so aufgezeigt werden, wie beispielsweise die Nutzung des Privatrezeptes zur Einschränkung des Medikamentenkonsums. Dies ist unter Verwendung eines Fragebogens kaum möglich, auch wenn der Bogen Platz für einen Freitext enthält, da Freitext-Fragen oft nicht beantwortet werden (Bendtsen et al. 1999).

Die Teilnehmerquote diese Arbeit von 39\% (17 von 43) liegt leicht über der, der oben erwähnten Studie zu Privatrezepten (33,9\%) (Hoffmann et al. 2014), jedoch deutlich unter den Quoten ausländischer Fragebogen-Studien zu Benzodiazepinen und Z-Substanzen. Beispielsweise hatten in einer englischen Arbeit 78,5\% (84 von 107) der eingeladenen Ärzte nach einmaliger Erinnerung den Fragebogen zurückgesandt (Siriwardena et al. 2010). Obwohl im Rahmen dieses Projekts der inhaltliche Fokus auf Privatrezepte weder im Anschreiben noch während der telefonischen Einladung explizit in den Vordergrund gerückt wurde - der Forschungstitel lautete „Hypnotika in der Praxis“ - sind BenzoZ in Deutschland offensichtlich eng mit dem Thema Privatrezepte für Kassenpatienten verknüpft. Da diese Verordnungsform einen juristischen Graubereich darstellt, könnte dies die Bereitschaft zur Studienteilnahme herabgesetzt haben, zusätzlich zu anderen Faktoren, wie Zeitmangel und bürokratischer Aufwand (Rosemann und Szecsenyi 2004; Hummers-Pradier et al. 2008).

Der Vergleich mit internationalen Interviewstudien zu einem ähnlichen Thema ist schwierig, da viele nicht über die Zahl der insgesamt kontaktierten Personen berichten (Cook et 
al. 2007; Rogers et al. 2007; Deans und Skinner 1992) und teilweise nur Ärzte zur Studienteilnahme einluden, die sich aktiv an der Studentenausbildung beteiligten, und denen somit ein höheres akademisches Interesse unterstellt werden könnte (Rogers et al. 2007). In der vorliegenden Arbeit wurden auf Grund des angestrebten theoretischen Samplings sowohl Lehrärzte der Universitätsmedizin Göttingen, als auch nicht universitär angebundene Hausärzte eingeladen und interviewt.

Die persönliche Kontaktaufnahme der interviewenden Ärztin zu potentiellen Studienteilnehmern hat sicherlich die Teilnahmebereitschaft gesteigert, wie bereits anderswo gezeigt werden konnte (Templeton et al. 1997). Die Relevanz, die Ziele der Studie und der zeitliche Aufwand konnten so direkt erklärt und Fragen beantwortet werden. Anrufe einer ärztlichen Kollegin wurden mit größerer Wahrscheinlichkeit vom Praxispersonal an den jeweiligen Arzt durchgestellt als die einer dritten Person, beispielsweise eines nicht-ärztlichen Wissenschaftlers oder einer Study Nurse. Ein weiter Grund für die zufriedenstellende Teilnahmebereitschaft ist möglicherweise die Tatsache, dass es sich bei der primären Rekrutierungsregion (Eichsfeld) um ein Gebiet handelt, dass - zwei Praxen ausgenommen - in anderen, parallel ablaufenden Studien des Instituts für Allgemeinmedizin der Universitätsmedizin Göttingen noch nicht eingeschlossen wurde, und die dort niedergelassenen Ärzte somit nicht mit Studienprotokollen oder -anfragen überlastet waren. Ebenfalls dürften die Tatsachen, dass es sich um einen einmaligen zeitlichen Aufwand von ca. 45 Minuten gehandelt hat und der Interviewort vom Interviewten bestimmt werden konnte, die Quote erhöht haben. Des Weiteren handelt es sich um ein für die hausärztliche Tätigkeit wichtiges Thema, was einen Förderfaktor zur Studienteilnahme darstellt (Peters-Klimm et al. 2013).

Nichts desto trotz konnten nicht alle niedergelassenen Allgemeinärzte oder hausärztlich tätige Internisten im primären Studiengebiet zu einer Teilnahme bewegt werden. Obwohl eine theoretischen Sättigung ohne Probleme erreicht werden konnte, muss aber in Betracht gezogen werden, dass ein Sampling Bias besteht und die dargestellten Meinungen und Erfahrungen von Ärzten stammen, die möglicherweise ein besonderes Interesse am Thema Schlaf- und Beruhigungsmittel haben, sich auf diesem Gebiet relativ kompetent fühlen auch wenn eine Unsicherheit bezüglich der Krankenkassenvorgaben in vielen Interviews um Ausdruck kam - und/oder Handlungsstrategien entwickelt haben, die sie für erfolgreich und richtig halten. Des Weiteren liegt zum jetzigen Zeitpunkt kein Feedback der Befragten zu der entwickelten Theorie vor. Auch können auf Grund der gewählten Methode keine für ganz Deutschland gültigen Angaben gemacht werden. Lediglich ermöglicht die Arbeit ein besseres Verstehen einer bis dato kaum erforschten Verhaltensweise. 


\subsection{Umsetzung der Methode}

Glasser und Strauss vertraten bei der Umsetzung der Grounded Theory zunächst ein rein induktives Vorgehen. Die Theorie sollte sich ausschließlich aus den erhobenen Daten generieren. Daher rieten sie beispielsweise von einer Literaturrecherche vor dem Eintritt in das Forschungsfeld ab, um nicht nur dem Prinzip der Offenheit, sondern auch dem der Fremdheit gerecht zu werden (Mey und Mruck 2009). Dies steht im Widerspruch zu der Tatsache, dass die Interviewende dieser Studie selbst Allgemeinmedizinerin und eben nicht fachfremd ist. Jedoch wird die Grounded Theory gegenwärtig als eine Methode angesehen, die auch deduktive Aspekte zulässt, insbesondere, wenn Vorkenntnisse zu einem besseren Gegenstandsverständnis beitragen - und entspricht damit der sonst üblichen Forschungsregel, sich zunächst über den Stand des Wissens zu informieren. Um dem Prinzip der Offenheit dennoch gerecht zu werden, wurden die Daten im Rahmen der Auswertung mit medizinischen Laien diskutiert.

Wie im Material- und Methodenteil bereits angedeutet, fand initial ein Cluster Samplingähnliches Vorgehen statt, d. h. es sollten möglichst alle Praxen im primären Rekrutierungsgebiet zur Teilnahme bewogen werden. Im Verlauf und nach Beginn der Auswertung erfolgte dann - so umsetzbar - ein theoretisches Sampling, also die Auswahl der Studienteilnehmer auf Grund der bis dato als relevant identifizierten Konzepte (Strauss und Corbin 2010). Ein ausschließlich theoretisches Sampling war im Rahmen dieser Arbeit kaum möglich, da im Vorfeld eines jeden Interviews nur einige Teilnehmerinformationen, wie Geschlecht, ungefähres Alter und Lage der Praxis bekannt waren. Im Gegensatz zu einer norwegischen Studie, die Apothekendaten vor dem Gespräch auswerteten und Ärzte in Viel-, Mittel- und Wenig-Verordnende einteilen konnte (Dybwad et al. 1997), war auf Grund der hiesigen ethik- und datenschutzrechtlichen Regelungen die jeweilige Verschreibungsmenge oder -methode nicht bekannt. Es erschien wenig zielführend, Teilnehmer beispielsweise mit dem Hinweis einzuladen, dass noch Ärzte gesucht werden, die nach eigener Einschätzung große Mengen Benzodiazepine und Z-Substanzen auf Privatrezept verordnen. Jedoch wurden Praxen im großstädtischen Gebiet nachrekrutiert, um den vermuteten Unterschied zwischen Stadt und Land herauszuarbeiten; auch die zunächst nicht geplante Ausverwertung der Diskussion im Qualitätszirkel geschah im Rahmen des theoretischen Samplings.

Auch wenn die Diskussionsinhalte des Qualitätszirkels sich teilweise mit den Aussagen der Einzelinterviews decken, erwiesen sich letztere zur Beantwortung der Forschungsfrage als wesentlich ergiebiger. So gab es Teilnehmerinnen des Zirkels, die viel von ihren Erfahrungen berichteten und ihre Meinung äußerten, während hingegen andere kaum das Wort ergriffen. Möglicherweise war dies einfach nur auf Grund unterschiedlicher Persönlichkeitstypen zu beobachten. Es wäre aber auch denkbar, dass - obwohl ein Qualitätszirkel dem offenen und kollegialen Austausch dienen soll - einzelne Teilnehmerinnen befürchteten, von Kolleginnen für ihr Verschreibungsverhalten oder ihre -methode kritisiert zu werden, oder eventuell vorhandene Unsicherheit bzw. Unwissenheit nicht zeigen wollten. Ebenfalls 
gestaltete sich die Teilstrukturierung wesentlich schwieriger, da der entwickelte Interviewleitfaden nur sehr eingeschränkt verwendet werden konnte, ohne die Diskussion zu unterbrechen. Dies hatte zur Folge, dass der Fokus des Gesprächs teilweise vom Thema abwich. Möglicherweise geschah dies auch, weil das BenzoZ-Privatrezept für die QZTeilnehmerinnen keine besonders hohe Relevanz hatte.

Bezüglich der Aufarbeitung und Darstellung der qualitativen Daten wurden alle von Tong et al. als relevant identifizierten Punkte im Rahmen dieser Arbeit beschrieben, wie aus der COREQ Checkliste (Anhang 8.7) hervorgeht (Tong et al. 2007).

\subsection{Einordnung in den aktuellen Forschungsstand}

Im Folgenden werden die Ergebnisse dieser Arbeit zunächst mit der in der Einleitung beschriebenen und weiterer Literatur zum Thema ärztliche Einstellung und Wahrnehmung von Benzodiazepinen und Z-Substanzen verglichen. Weiterhin wird die Ambivalenz als zentrales Phänomen des zuerst dargestellten übergeordneten paradigmatischen Modells diskutiert. Abschließend werden die Erkenntnisse zur Verwendung des Privatrezepts sowie das zweite paradigmatische Modell mit dem zentralen Phänomen Kreieren einer Hemmschwelle in den aktuellen Forschungsstand eingeordnet.

\subsubsection{Einordnung in den Forschungsstand zur Einstellung von Ärzten}

Berücksichtig man nur die Teilnehmer der Einzelinterviews, nahmen sich mehr Männer $(59 \%)$ als Frauen (41\%) Zeit für die Studie. Interessanterweise findet sich genau dieselbe Verteilung in der Umfrage von Hoffmann zum Thema BenzoZ-Privatrezept (Männer 59,4\%, Frauen 40,6\%) (Hoffmann et al. 2014), wobei auf Grund des qualitativen Designs dieser Arbeit und der somit niedrigen Teilnehmerzahl schon eine weitere Ärztin das Verhältnis um 3\% zugunsten der Frauen verschoben hätte. Dass insgesamt mehr Männer als Frauen an Studien teilnehmen, wurde bereits anderswo beschrieben. Jedoch können Beobachtungen, nach denen Männer mehr Benzodiazepine und Z-Substanzen verordnen (Monette et al. 1997; Davidson et al. 1994; Bjørner und Laerum 2003) oder Frauen mehr Schlaf- und Beruhigungsmittel an Frauen verschreiben (Morabia et al. 1992), nicht durch diese Arbeit bestätigt werden. Beide Geschlechter berichteten im gleichen Masse über die Verwendung dieser Medikamente.

Wie unter 6.2. bereits erwähnt, ist die tatsächlich durch die Teilnehmer verschriebene BenzoZ-Menge unbekannt. Daher lässt sich keine eindeutige Assoziation zwischen dem Alter des Arztes bzw. seiner Ausbildung (Arzt in Weiterbildung, Facharzt für Allgemeinmedizin oder praktischer Arzt) und der Verschreibungsmenge feststellen. Es zeigte sich auch keine Assoziation zwischen dem Alter bzw. der Ausbildung und der berichteten Häufigkeit der Nutzung. Allerdings gaben schon länger niedergelassene Teilnehmer sowohl in den Ein- 
zelinterviews als auch in der QZ-Diskussion an, dass früher mehr Schlaf- und Beruhigungsmittel verordnet worden seien.

Diese Aussagen decken sich daher nur bedingt mit der Feststellung von Subelj et al., das viel-verschreibende Ärzte älter sind (Subelj et al. 2010). Auch aus methodischen Gründen ist hier keine Bestätigung oder Entkräftung möglich: Qualitative Beobachtungen und Aussagen sind nicht verallgemeinerbar, und es wurden keine tatsächlich getätigten Verordnungsmengen erhoben. Vielmehr lässt sich feststellen, schon länger Niedergelassene eine Zeit miterlebt haben, in der die Nebenwirkungen der Medikamente - besonders der ZSubstanzen - noch nicht hinreichend erforscht oder beachtet waren und diese Mittel häufiger genutzt wurden. Dass nicht das Alter des Arztes, sondern vielmehr das Alter des Patienten entscheidend dafür ist, ob ein BenzoZ verordnet wird, konnte an anderer Stelle gezeigt werden (Donoghue und Lader 2010; Isacson et al. 1993). Je älter ein Patient ist, desto wahrscheinlicher erhält er ein entsprechendes Medikament. Isacson et al. kamen sogar zu dem Ergebnis, dass Alter ein besserer Prädikator für eine hohe Verschreibungsmenge ist als das Geschlecht. Nichtsdestoweniger erhielten in Deutschland im Jahr 2015 Frauen 56\% mehr Psychopharmakaverordnungen als Männer (Glaeske 2017).

Der von den Teilnehmern dieses Projektes als stereotypisch wahrgenommene Patient weiblich, älter und oft im Pflegeheim lebend - entspricht im Wesentlichen der Studienlage, wie in der Einleitung beschrieben (Subelj et al. 2010; Boixet et al. 1996; Cheung et al. 2014). Dabei wurde der in der Literatur im Zusammenhang mit BenzoZ verwendete Begriff des deserving patient nicht von den Befragten genutzt. Dieser bezeichnet einen Patient, der ein solches Medikament verdient hat und der sich - neben den oben genannten Attributen des Stereotypen - durch eine langjährige Verordnung durch andere Ärzte auszeichnet (Sirdifield et al. 2013) Allerdings gebrauchten die Ärzte oft die Bezeichnung des geerbten Patienten, die sich in anderen Arbeiten vorwiegend auf Opiate bezieht (Owston 2016; Gourlay und Heit 2009).

So wurden in den vergangenen Jahrzehnten u. a. in den USA Präparate wie Oxycordon oder Hydromorphon nicht nur an postoperative und schwerkranke Patienten mit eingeschränkter Lebenserwartung abgegeben, sondern zunehmend auch an jüngere Menschen mit chronischen Erkrankungen wie Rückenschmerzen. Dies führte in Folge zu vielen Suchtproblemen; so waren in den Vereinigten Staaten im Jahr 2009 2,3 Millionen Menschen entweder von Heroin oder einem verschreibungsfähigen Opiat abhängig (Substance Abuse and Mental Health Services Administration 2010). Auch Überdosierungen mit eben diesen verschreibungsfähigen Stoffen können zunehmend beobachtet werden (Paulozzi et al. 2011). Aber auch in Deutschland sind Opiatverordnungen zwischen 2006 und 2015 um 30\% angestiegen. Es wird geschätzt, dass hierzulande 200.000-300.000 Menschen von diesen starken Schmerzmitteln abhängig sind (Glaeske 2017). Im Falle eines Arztwechsels finden sich somit ähnliche Situationen, wie von den Teilnehmern dieser Studie beschrie- 
ben. Das Medikament ist womöglich nicht (mehr) indiziert, ein Absetzen ist auf Grund der entstandenen Abhängigkeit aber denkbar schwierig.

Der von den Teilnehmern verwendete Begriff der Suchtstruktur findet sich weder in den Anfangs erwähnten Leitlinien zur Unipolaren Depression, zu Angststörungen, zum nichterholsamen Schlaf noch zur Insomnie (S3-Leitlinie Unipolare Depression 2017; S3-Leitlinie Behandlung von Angststörungen 2014; S3-Leitlinie Nicht erholsamer Schlaf/Schlafstörungen 2009, 2017; S1-Leitlinie Insomnie 2012). Auch die Internetseite der Arbeitsgemeinschaft der Wissenschaftlichen Medizinischen Fachgesellschaften bringt mit diesem Ausdruck keine Leitlinie in Verbindung (AWMF 2018). Unter „dependence structure“ finden sich bei Pubmed über 20.000 Einträge, unter „dependence structure hypnotics" 58, die sich aber zumeist mit biochemischen Prozessen befassen, wie die Arbeit von Souza-Pinto et al. zum Thema Diazepam-Entzug (Souza-Pinto et al. 2007). Im Artikel finden sich die beiden Wörter jedoch nicht als zusammenhängender Begriff. Mit Hilfe von Google lassen sich einige Internetreferenzen zur Suchtstruktur identifizieren, aber ohne genaue oder einheitliche Definition. Allerdings wird in diesem Zusammenhang oft das Werk von Bilitza genannt (Bilitza 2009, Seite 14), das nicht in Pubmed gelistet ist und in dem unter „Psychodynamik der Sucht“ die Entstehung einer Sucht anhand von fünf Punkten beschrieben wird - angefangen von „(1) Biographie, Lebensgeschichte und psychische Entwicklungsbedingungen“ bis hin zu „(5) im Fall der Fehlanpassung durch Konsum chemischer Substanzen als Suchtentwicklung und schließlich Suchtstruktur mit ihren jeweiligen Symptomen (...). Psychodynamik der Sucht beschreibt folglich Prozess und Struktur der Sucht, das heißt die dynamischen Prozesse der Suchtentstehung und die daraus entstandenen psychischen Suchtstrukturen.“ Die interviewten Ärzte nutzen den Begriff jedoch in einem breiteren Kontext, der sich nicht nur auf psychische (Verhaltens-) Muster beschränkt, sondern sich auch über sozioökonomische Faktoren definiert, beispielsweise ein niedriges Bildungsniveau (Vergleiche 5.2.6.2). Im Gegensatz zu Bilitza wurde Patienten nicht nur eine Suchtstruktur zu geschrieben, wenn sie bereits süchtig geworden waren, sondern wenn das jeweilige Verhalten und der sozioökonomische Kontext des Patienten laut Meinung der Interviewten darauf hinwiesen, dass diese Person schneller abhängig werden würde als eine andere.

Die von den Befragten genannten Gründe für die Verschreibung von Benzodiazepinen und Z-Substanzen decken sich nur teilweise mit denen von Bjørner und Kjølsrød identifizierten, beispielweise Angststörungen und akute Krisensituationen (Bjørner und Kjølsrød 2002). Auch die gelegentliche Weiterführung auf Grund einer schwierigen sozialen Situation finde sich in beiden Arbeiten. Eine primäre Insomnie war für einige Teilnehmer dieser Studie jedoch kein Grund, ein solches Medikament zu verordnen. Eine Verschreibung auf Grund von Schmerzen berichtete keiner der Befragten. Diese Medikamentenverwendung deckt sich zum Teil mit den unter 2.2. beschrieben aktuellen Leit- und Richtlinien. So können Benzodiazepine bei akuten und schweren Angst- und Panikattacken verabreicht werden (Leitlinie Angststörungen 2014); eine dauerhafte Verschreibung auf Grund der sozialen 
Verhältnisse entspricht aber nicht den momentanen Bestimmungen. Und auch die Bestätigung der Angst- und Panikstörung durch einen weiteren Facharzt macht eine langfristige BenzoZ-Einnahme nicht automatisch und in allen Fällen leitlinienkonform, da diese Substanzen mittlerweile als psychiatrischen Reservemedikamente dienen sollen. Jedoch wird so die Verantwortung für das Aufrechterhalten der Verschreibung übertragen, wie im nächsten Absatz erläutert. Als Hypnotikum können Benzodiazepine und Z-Substanzen laut der AMRL zwar vier Wochen verwendet werden (Gemeinsamer Bundesausschuss 2016), jedoch spricht sich die deutsche Gesellschaft für Schlafforschung und Schlafmedizin für eine nicht-medikamentöse Therapie von kurzfristigen Schlafstörungen aus (S3-Leitlinie Nicht erholsamer Schlaf/Schlafstörungen 2017, 2009), was sich mit der Vorgehensweise einiger Ärzte deckt. Die von Bjørner und Kjølsrød festgestellte Nutzung als Schmerzmittel (Bjørner und Kjølsrød 2002) ist in Deutschland obsolet und wurde in keinem Interview angegeben.

Viele der Befragten wiesen darauf hin, dass sie längerfristig nur bei einer „klaren Indikation" ein Benzodiazepin oder eine Z-Substanz verordnen würden. Hierunter wurde eine psychiatrische Erkrankung verstanden, die teilweise durch einen Neurologen oder Psychiater bestätigt werden sollte oder nach Angaben der Befragten bestätigt wurde. Dies ist zwar nur bedingt leitliniengerecht, da Z-Substanzen nur als Schlafmittel zugelassen sind und Benzodiazepine mittlerweile, wie erwähnt, nur noch als Reservemedikamente bei z. B. Angststörungen oder Depressionen gelten (S3-Leitlinie Behandlung von Angststörungen 2014; S3-Leitlinie Unipolare Depression 2017). Jedoch deckt sich dies mit den Ergebnissen einer schwedischen Studie, die feststellte, dass ein Langzeitgebrauch häufig mit einer den DSM-IV Kriterien entsprechenden Diagnose einhergeht (Zandstra et al. 2004), widerspricht aber der Datenauswertung der US-amerikanischen, ambulanten Versorgungsumfrage von fast 99.000 Besuchen (Maust et al. 2016). Hier zeigte sich, dass ein Großteil der Langzeit-Verschreibungen eher auf Grund eines Symptoms als auf einer psychiatrischen Diagnose stattfand. Die Autoren schlossen daraus, dass viele Verordnungen im Rahmen einer kurzfristigen Stresssituation oder Anpassungsstörung begonnen wurden, die an sich keine Diagnose darstellten und durch das Symptom beschrieben wurde, und dass sich daraus eine dauerhafte Medikamenteneinnahme entwickelte.

Sowohl die QZ- als auch die Teilnehmer der Einzelinterviews nahmen insgesamt medikamentöse und nicht-medikamentöse Therapien im Vergleich zu BenzoZ als weniger effektiv wahr, auch wenn einzelne Personen gute Erfahrungen mit bestimmten pflanzlichen Mitteln, Antidepressiva oder Interventionen, wie einem Schlaftagebuch gemacht hatten. Bezüglich der Risiken sahen die Teilnehmer dieser Studie vor allem das Abhängigkeits- und Suchtpotential. Dies deckt sich mit den Ergebnissen von Everitt et al. (Everitt et al. 2014). Interessanterweise werden fassbare Probleme, wie Stürze oder Einschränkung der Vigilanz mit möglicherweise resultierendem Verlust kognitiver Fähigkeiten weitaus weniger oder nur im Zusammenhang mit einer Anhängigkeit gesehen. 
Eine Übertragung der Verantwortung zeigt sich in Rahmen dieses Projektes insbesondere bezüglich geerbter Patienten. Kausal wird in diesen Fällen der vorherige Arzt für die Entstehung der Sucht verantwortlich gemacht wird. Dabei bezieht sich dies in der Regel nicht speziell auf mitbehandelnde Psychiater, wie in anderen Arbeiten beschrieben (Sirdifield et al. 2013; Rogers et al. 2007), sondern deckt sich mit dem Ergebnis von Siriwardena et al., dass ehemalige Hausärzte als Verantwortliche betrachtet werden (Siriwardena et al. 2010). Die Verantwortung für die Aufrechterhaltung der Verschreibung und damit der Abhängigkeit wird jedoch oft an einen Neurologen oder Psychiater abgegeben, der die Therapie mit einem BenzoZ bestätigt oder empfohlen hat.

\subsubsection{BenzoZ-Verordnung im ländlichen und städtischen Gebiet}

Die Vermutung der auf dem Land niedergelassenen Ärzte, dass Kollegen in der Stadt mehr über Schwierigkeiten im Umgang mit Benzodiazepinen und Z-Substanzen berichten würden, ließ sich durch die Nachrekrutierung und Durchführung von Interviews im großstädtischen Raum nicht bestätigen. Ärzte im großstädtischen Bereich empfanden die Lage ihrer Praxis diesbezüglich nicht als Nachteil. Sie gaben an, dass sie auf Grund der örtlichen Drogenszene zu einem konsequenten und strengen Verschreibungsverhalten gezwungen wären, was zur Folge hätte, dass sich kaum Problem im Alltag bezüglich dieser Medikamente ergeben würden. Dieser Einschätzung liegen aber keine objektiven Verordnungsdaten zugrunde, wie der Studie von Isacson et al.: Sowohl in der Großstadt als auch auf dem Land war dort die pro Rezept verschriebene BZD-Menge geringer als im kleinstädtischen Bereich (Isacson et al. 1993). Als mögliche Erklärung gaben die Autoren „kulturell und geographische Unterschiede“ an, die für die geringere Verschreibungsmenge auf dem Land verantwortlich sein könnten; ebenso wie die Tatsache, dass die Datenerhebung pro Arzt erfolgte und nur wenige Ärzte längere Zeit in der schwedischen Provinz arbeiten wollten. Da in dieser Studie die Menge pro Rezept erfasst wurde, wäre es möglich, dass Patienten in der Stadt sich auf Grund der leichteren oder kürzeren Anfahrt einfach häufiger in der Praxis vorstellten. Dieser Punkt wurde von Isacson et al. jedoch nicht aufgegriffen; sie kamen zu dem Schluss, dass Ärzte im städtischen - insbesondere im großstädtischen - Gebiet häufiger unbekannte Patienten sehen und daher, wenn überhaupt, kleinere Packungen verordnen würden. Die Aussagen der Teilnehmer der vorliegenden Studie gehen diesbezüglich weiter: Es soll sich in der Drogenszene herumsprechen, dass von diesem Arzt kein Rezept für Schlaf- und Beruhigungsmittel zu erwarten sei. Wer konsequent sei, vermeide Probleme in der Zukunft, wie unter 5.2.7.3 dargestellt. Dies sei in der Stadt wichtiger als auf dem Land. Des Weiteren ist die strikte Ablehnung einer Verordnung möglicherweise leichter, wenn keine langjährige Beziehung zum Patienten und/oder dessen Familie besteht.

\subsubsection{Einordnung in den Forschungsstand zur Ambivalenz}

Der Schweizer Psychiater Eugen Paul Bleuler (1857-1939) verwendete den Begriff „Ambivalenz" erstmals öffentlich 1910 im Rahmen eines Vortrags (Sponsel 2002); ein Bericht 
hierzu erschien im Folgejahr (Riklin 1911). Vollständig wurde der Vortrag jedoch erst 1914 abgedruckt (Bleuler 1914). Für Bleuler gab es drei Formen der Ambivalenz, die sich auf das gleichzeitige Vorhandensein von widersprüchlichen Gefühlen (affektive Ambivalenz), Wünschen (voluntäre Ambivalenz) und Beurteilungen (intellektuelle Ambivalenz) beziehen. Die einzelnen Formen lassen sich nicht trennen und gehen ineinander über. Des Weiteren deutete Bleuler die Ambivalenz als ein Hauptsymptom der Schizophrenie, da der Erkrankte beide Seiten nebeneinander bestehen lässt, während der Nicht-Schizophrene meistens ein Fazit zieht. Später fand der Begriff Verwendung in der Psychologie (Jonas et al. 2000; Thomae 1960) und Soziologie (Lüscher 2009); heute definiert ihn der Duden als „Zwiespältigkeit, Spannungszustand, Zerrissenheit [der Gefühle und Bestrebungen]“ (Duden 2018).

In den Ergebnissen dieser Arbeit zeigt sich Ambivalenz als zentrales Phänomen, auf das die Ärzte mit verschiedenen Handlungsstrategien, u. a. dem Ausstellen eines BenzoZPrivatrezeptes reagieren. Auf der einen Seite kann dem Patienten so ein wirksames Medikament, nach dem er eventuell sogar verlangt, verschrieben werden. Auf der anderen Seite tut der verordnende Arzt etwas dafür, die konsumierte Menge und damit auch die Nebenwirkungen gering zu halten, indem er die finanzielle Belastung als Hemmschwelle einsetzt und kann somit beiden entgegengesetzten, ambivalenten Gefühlen gerecht werden.

Flick et al. beschrieben in ihrer Arbeit drei verschiedene Verschreibungsmuster bezüglich Schlaf- und Beruhigungsmitteln; neben der Verordnung ,auf Anforderung“ und der „wohl überlegten Verordnung“ zeigte sich hier als drittes Muster auch ein „ambivalentes Verhalten“. So waren sich die Teilnehmer der Studie - genau wie die Befragten dieser Arbeit bewusst, dass Benzodiazepine und Z-Substanzen nur für kurze Zeit verordnet werden sollten und ein Mittel der „letzten Wahl“ darstellen, sahen aber oft keine Alternative. Laut den Autoren fiel es den Ärzten in dieser dritten Gruppe schwer, sich einzugestehen, welchen Stellenwert diese Medikamente in ihrer täglichen Arbeit haben und dass sie weitaus öfter als nur in Notfallsituationen verwendet werden (Flick et al. 2012). Letzteres deckt sich insofern mit den Ergebnissen dieses Projektes als die BenzoZ-Verwendung in Krisensituationen häufig genannt wurde. Allerdings fehlen objektive Daten zur Verschreibungsmenge, um die Aussage von Flick bezogen auf diese Studie zu untermauern. Das „Dilemma“ der Ärzte, eigentlich kein Schlaf- oder Beruhigungsmittel verschreiben zu wollen und es trotzdem tun, wurde auch schon in älteren Studien beschrieben (Bendtsen et al. 1999; Bradley 1992a). Bradley bezeichnete dies als „kognitive Dissonanz“. Bendtsen et al. stellten fest, dass Ärzte Leitlinien insbesondere nutzen, um zukünftige Problemfälle zu vermeiden. Folglich würde eine Anpassung der Leitlinien nicht zu einer Lösung aktuell schwieriger Verschreibungsentscheidungen führen. Dies lässt sich insofern auf die vorliegende Arbeit übertragen, als das den Teilnehmern zwar nicht der Wortlaut, jedoch der wesentliche Inhalt der aktuellen AMRL bekannt war, dies aber keine gute Orientierungshilfe darstellte, wenn Patienten schon lange Benzodiazepine oder Z-Substanzen einnahmen. 
Eine ähnliche Situation findet sich heutzutage bei der Verschreibung von Opiaten für chronische, nicht maligne Erkrankungen. Opiate besitzen - genau wie Benzodiazepine und Z-Substanzen - ein deutliches Abhängigkeitspotential, auch wenn manche Ärzte das weniger kritisch sehen, wie das nachfolgende Zitat aus dem Artikel von Seamark et a. zeigt: „I think my brain tells me that there is no problem, although you know, life in general makes you think that you must be a bit careful but I don't think there is a reason why we shouldn't put somebody on long-term opiates if the need is there.“ „I think there's a lot of unreasonable fears, the biggest one being addiction and I think it's a grossly, grossly overstated concern, addiction. In my practice I've yet to see the patient who was put on opiates for benign pain who is addicted.“ (Seamark et al. 2013, Seite 823 \& 824). In einer anderen Studie aus 2014 waren 82\% der Teilnehmer bezüglich einer Abhängigkeitsentwicklung besorgt, und jüngere Ärzte zeigten sich zurückhaltend bei der Verordnung von Opiaten (Jamison et al. 2014). Folglich findet sich auch hier eine deutliche Ambivalenz in der ärztlichen Wahrnehmung, denn unumstritten ist, dass Opiate stark wirksame Schmerzmittel sind.

\subsubsection{Einordnung in den Forschungsstand zum Privatrezept}

Wie einleitend bereits erwähnt, ist zum Thema Schlaf- und Beruhigungsmittel auf Privatrezept wenig bekannt. Die Nutzung dieser Rezeptform, um die BenzoZ-Einnahme eines Patienten zu minimieren, ist nach den Kenntnissen der Autorin bisher nicht beschrieben und wird unter 6.4 ausführlich diskutiert. Bezüglich Diagnose und Problemdauer lässt sich feststellen, dass die Einschätzung der von Hoffmann et al. befragten Hausärzte und Apotheker, Patienten mit Langzeitgebrauch erhielten häufiger ein Privatrezept (Hoffmann et al. 2014), sich nicht ganz mit den oben dargestellten Ergebnissen deckt. Sofern diese Patienten eine psychiatrische Erkrankung haben, erhalten sie durchaus auch langfristig ein BenzoZKassenrezept. Allerdings finden sich insbesondere unter den geerbten Patienten viele mit einer Schlafmittelabhängigkeit, deren Konsum durch ein Privatrezept begrenzt werden soll.

Die Substanzklasse (Benzodiazepin oder Z-Substanz) spielt bei der Wahl der Rezeptformkeine unmittelbare Rolle; viel entscheidender ist, aus welcher Indikation das Medikament verwendet werden soll. Hierbei kommt es oft zu einem Gebrauch außerhalb der Zulassung, also einen Off-Label Use, da vereinzelt die ausschließlich als Schlafmittel zugelassenen ZSubstanzen zur Therapie von psychiatrischen Erkrankungen genutzt werden. Ist dies der Fall, erhalten Patienten hierfür ein Kassenrezept. Werden Z-Substanzen als „reines“ Schlafmittel oder „Life-Style“-Medikament verschrieben, geschieht dies oft auf Privatrezept. Berücksichtig man allerdings die Auskünfte der AOK-Beratungsapotheke, kommt es jedoch insbesondere bei den Benzodiazepinen oft zu einen Off-Label Gebrauch, da beispielweise das häufig verwendete Tavor ${ }^{\circledR}$ (Lorazepam) gar nicht zur Behandlung isolierter Schlafstörungen zugelassen ist. Dies deckt sich mit der Darstellung von Nissen et al., die Lorazepam ebenfalls nicht als zur Behandlung von isolierten Schlafstörungen geeignet aufführt (Nissen et al. 2014). Die Fachinformation des Medikaments besagt nichts Gegenteili- 
ges, also gibt das Medikament auch nicht als primäres Hypnotikum aus, allerdings muss der Text genau gelesen werden. Hier wird als Anwendungsgebiet die „symptomatische Kurzzeitbehandlung von Angst-, Spannungs- und Erregungszuständen sowie dadurch bedingten Schlafstörungen“" angegeben (Pfizer 2016).

Der direkte Patientenwunsch nach einem Schlaf- bzw. Beruhigungsmittel erscheint bezüglich der Wahl der Rezeptform weniger wichtig; dies war für die Teilnehmer der Einzelinterviews eher ein Grund, ein Rezept - egal, ob Kasse oder privat - zu verweigern. Natürlich könnte es sich hierbei auch - und insbesondere gegenüber einer ärztlichen Kollegin um die behandlungs- und gesundheitspolitisch korrekte und erwartete Antwort gehandelt haben, die möglicherweise vom Verhalten im Alltag abweicht. Die Verordnung eines BenzoZ ohne medizinische Indikation könnte zu Recht als Wunschverordnung betrachtet werden, obwohl dieser Begriff von keinem der Teilnehmer im Interview verwendet wurde. Im Qualitätszirkel wurden medizinisch nicht notwendige Schlaf- und Beruhigungsmittel als „Lifestyle Medikamente“ bezeichnet und klar privat rezeptiert. Insofern decken sich die Ergebnisse der Arbeit teilweise mit der Annahme von Hoffmann et al., BenzoZPrivatrezepte würden ausgestellt, weil der Patient die Verschreibung wünscht (Hoffmann et al. 2014). Dass Patienten, die sich ihrer Sucht bewusst sind, explizit ein Privatrezept wünschen, um als Versicherte nicht bei ihrer jeweiligen Krankenkasse aufzufallen, wie in einem Beitrag von Gerd Glaeske im DHS Jahrbuch Sucht 2017 berichtet (Glaeske 2017), wurde in keinem der Interviews erwähnt. Allerdings könnte eine Studie zur Patientenperspektive diese Annahme sicherlich wiederlegen oder untermauern.

Die Verschreibungsentscheidung und nachgeordnet die Wahl der Rezeptform wird beeinflusst bzw. erklärt durch den wahrgenommenen Patiententyp. „Brave“ Patienten, die kleine Menge einnehmen und mit ihrem Verhalten den Erwartungen des Arztes entsprechen, erhalten eher ein Kassenrezept. Patienten, die diese Erwartungen nicht erfüllen, oder unbekannten Patienten wurde eher ein Privatrezept ausgestellt. Somit gibt es auch in Bezug auf die Frage ,Privat- oder Kassenrezept?‘ einen deserving patient - also einen Patienten, der ein Kassenrezept verdient hat. Auch wenn sich andere Studien nicht speziell auf die Rezeptform beziehen, sondern auf die generelle Frage, wann ein Schlaf- und Beruhigungsmittel verschrieben wird (Rogers et al. 2007; Sirdifield et al. 2013), sind die Ergebnisse teilweise deckungsgleich. So wurden auch im Rahmen dieser Arbeit Patienten mit einer Alkoholoder Drogenabhängigkeit als undeserving bewertet und erhielten eher ein Privatrezept, wie das Zitat unter 5.3.4 zeigt.

Zwei Teilnehmer stellten einigen Patienten auf Grund ihrer finanziell schlechten Situation ein Kassenrezept aus, während andere Patienten Privatrezepte erhielten. Dies war jedoch die Ausnahme, da Benzodiazepine und Z-Substanzen preiswerte Medikamente sind und die Selbstzahlung von den Ärzten als dem Patienten zumutbar und erschwinglich empfunden wurde. Dem entsprechend spielte die Entlastung des Praxisbudgets durch BenzoZPrivatrezepten sowohl in der Arbeit von Hoffmann et al. als auch in dieser Studie eine un- 
tergeordnete Rolle (Hoffmann et al. 2014), obwohl einige Befragte dies als Grund für die Verwendung angaben. Auch an dieser Stelle zeigt sich das ambivalente und nicht ganz konsistente Verhalten der Teilnehmer, da einerseits die finanzielle Belastung des Patienten als gering eingestuft wird, auf der anderen Seite das Ziel verfolgt wird, mittels dieser Kosten eine Verhaltensänderung zu erreichen. Andere Ärzte betonten, dass „die Versichertengemeinschaft" nicht für vom Patienten gewünschte Verordnungen aufkommen soll und lieBen dabei außer Acht, dass dieser auf Grund der entstandenen Sucht gar nicht mehr anders kann als sich das Medikament zu „wünschen“. Aber auch bei einer erkannten Abhängigkeit wurde mit dem gleichen Argument - Entlastung der Versichertengemeinschaft - unter Umständen ein Privatrezept genutzt. Sicherlich spielen oft mehrere Beweggründe eine Rolle und so könnte gerade in Bezug auf diese abhängigen Patienten die Hoffnung oder der Wunsch bestehen, den Konsum durch ein Privatrezept einzuschränken.

Fragt man nach der aktuellen Arzneimittelrichtlinie, ist der genaue Wortlaut nahezu unbekannt, jedoch gaben so gut wie alle Teilnehmer bezüglich ihres Umgangs mit Schlaf- und Beruhigungsmitteln an, dass diese nur kurzfristig verschrieben werden sollten und, dass eine längere Verschreibung gut begründet werden muss. Dies deckt sich im Wesentlichen mit den Vorgaben der AMRL und bezüglich des generellen Umgangs mit Schlaf- und Beruhigungsmitteln scheint wenig Unsicherheit zu herrschen. Angemerkt sei aber, dass ein Abfragen von Wissen während der Interviews, beispielsweise bis zu wieviel Wochen Hypnotika laut der AMRL verschrieben werden dürfen, bewusst vermieden worden ist, um eine Gesprächsatmosphäre ohne Prüfungscharakter zu schaffen, die das offene Thematisieren der Privatverordnungen für Kassenpatienten ermöglichte. Des Weiteren war nicht allen Teilnehmern der Unterschied zwischen Leitlinien (nicht bindend) und Richtlinien (bindend) bekannt (Vergleiche 5.2.6.1). Auch dies wurde aus dem oben genannten Grund nicht näher eruiert.

Sowohl die AOK als auch die BARMER beziehen sich bezüglich Hypnotikaverordnungen auf die Anlage III der Arzneimittelrichtlinie des Gemeinsamen Bundesausschusses. Die Ärzte bewerteten aber die Vorgaben der AMRL und die der Krankenkassen getrennt voneinander. Und wie die Ergebnisse zeigen, ist es weniger die Unklarheit über die aktuelle Arzneimittelrichtlinie als über die Vorgaben der GKV, die zu einem vermehrten Gebrauch von Privatrezepten führt. Die Vermutung, die Rezeptform würde gewählt werden, um durch Intransparenz der Kontrolle durch die Krankenkassen zu entgehen und sich dort nicht für sein eigenes Verschreibungsverhalten rechtfertigen zu müssen (Glaeske 2014), bzw. das „Nichterscheinen in den Kassendaten“ (Hoffmann et al. 2014) zu erzielen, wurde in dieser Studie insofern bestätigt, als Unsicherheit darüber besteht bei welcher Indikation und wie lange Benzodiazepine und Z-Substanzen von den gesetzlichen Krankenkassen bezahlt werden. Auch wenn Nachfragen der Krankenkassen selten sind, äußerten mehrere der Befragten Sorge vor einem Regress (Vergleiche 5.2.7.2 und 5.3.9). 
Bevor im Folgenden die beiden zentralen Punkte - die Konsumeinschränkung durch das Privatrezept und die fehlenden Orientierungshilfen, die diese Rezeptform begünstigen ausführlicher aufgegriffen werden, bleibt festzustellen, dass in Deutschland das BenzoZPrivatrezept für kassenärztlich Versicherte ein Stück weit zu einer Routine geworden ist, die im Alltag nicht mehr hinterfragt wird. Viele Patienten sind mittlerweile nicht nur an das Schlaf- und Beruhigungsmittel gewönnt, sondern auch daran, dieses selber bezahlen zu müssen. Dies fördert die Fortsetzung dieser Verschreibungspraxis.

\subsection{Das Privatrezept zur Konsumeinschränkung?}

Wie im Ergebnisteil dieser Arbeit dargestellt, erleben Hausärzte kaum Patientenbeschwerden bezüglich BenzoZ-Privatverordnungen. Wahrscheinlich ist dies der Tatsache geschuldet, dass Patienten nicht wissen, welches Medikament wie lange auf ein Kassenrezept aufgeschrieben werden kann, wie bereits erwähnt, besteht hierzu bezüglich Schlaf- und Beruhigungsmittel unter den Ärzten schon eine erhebliche Unsicherheit. Möglicherweise sind sich aber auch einige Patienten sehr bewusst, dass sie nicht mehr ohne ihr Schlafmittel auskommen, und haben in der Vergangenheit Versuche des Arztes erlebt, dass Präparat zu reduzieren oder abzusetzen. Entsprechend dem unter 5.3.3.2 dargestellten Zitat Weil die Leute sind ja einfach glücklich, wenn's ibnen denn wer aufschreiben würde, ne? (110,79) akzeptieren Patienten die Selbstzahlung ohne Beschwerden, da der Arzt diesbezüglich „am längeren Hebel sitzt" und eine Verordnung auch ablehnen könnte.

Hinzukommend werden einige Patienten vom Arzt nicht als medikamentenabhängig wahrgenommen, insbesondere bei Vorliegen einer Niedrigdosisabhängigkeit ohne Eskalation der Einnahmemenge oder -frequenz. Das Beibehalten eines Einnahmeschemas wird sogar oft als Erfolg der Handlungsstrategie Privatrezept angesehen und nicht als Förderung bzw. Aufrechterhaltung der Abhängigkeit.

In der Literatur findet sich die Idee der unmittelbaren Suchtkontrolle durch finanzielle Kosten für den Süchtigen im Zusammenhang mit dem Rauchen von Zigaretten. Zwar ergab eine Umfrage unter Rauchern, dass bei einer theoretischen Preiserhöhung ein Großteil versuchen würde aufzuhören oder weniger zu rauchen, allerdings wollte ein nicht unerheblicher Teil der Befragten einfach zu einer günstigeren Zigarettenmarke wechseln (Gigliotti et al. 2014). Eine andere Studie ergab, dass Zigaretten stangenweise eingekauft oder online bestellt wurden, um trotz Preiserhöhung die Konsummenge gleich zu halten (Xu et al. 2013). Somit scheinen finanzielle Kosten bei bestehender Abhängigkeit kein Garant für eine Konsumeinschränkung zu sein. Ähnliche Strategien zur Kostensenkung wären auch im Zusammenhang mit Schlafmitteln möglich. Zwar können über die Internetapotheke DocMorris keine Benzodiazepine oder Zolpidem geordert werden. Zopiclon ist jedoch von mehreren Firmen verfügbar. Der Preis für 20 Tabletten à 7,5 mg beträgt 15,10 Euro (0,76 Euro pro Tablette). Dies deckt sich mit der Preisauskunft der Göttinger Humboldt Apotheke. Jedoch erhalten Neukunden bei DocMorris einmalig einen Gutschein von 10 Euro; 
als Dauerbonus bietet das Portal 2,50 Euro für jedes rezeptpflichtige Medikament - egal, ob Kassen- oder Privatrezept (DocMorris 2018).

Des Weiteren werben im Internet zahlreiche dubiose Händler damit, Medikamente auch ohne Rezept zu verkaufen, wie beispielsweise die Seite medizin-ohne-rezept.com. Zwar liegt der Preis für 20 Tabletten Zopiclon à 7,5 mg mit 29,90 Euro (1,50 Euro pro Tablette) über dem von DocMorris und der örtlichen Apotheke; es werden aber auch Großpackungen unterschiedlicher Hersteller angeboten, beispielsweise 200 Tabletten à 7,5 mg zu 99,90 bis 118,80 Euro (0,50-0,59 Euro pro Tablette). Neben weiteren Z-Substanzen, wie Zaleplon und Zolpidem, bietet die Seite 13 verschiedene Benzodiazepine inklusive Flunitrazepam und acht verschiedene Schmerzmittel, darunter Ketamin, Hydrocodon und Fentanyl an - angeblich alles ohne Rezept (Medizin ohne Rezept 2018). Ein Impressum ist jedoch auf der optisch professionell erscheinenden Internetseite nicht zu finden, vor der an anderer Stelle ausdrücklich gewarnt wird - sowohl in Bezug auf die medizinischen Risiken als auch bezüglich eines Betruges (Onlinewarnungen 2018).

Bezüglich Schlaf- und Beruhigungsmitteln gibt es international einige Studien, die sich mit Verordnungen dieser Medikamente in Abhängigkeit von der Erstattung durch die Krankenkassen beschäftigen. So werden in den Niederlanden seit dem 01.Januar 2009 Benzodiazepine nicht mehr bezahlt, wenn sie als Schlaf- oder Beruhigungsmittel verschrieben werden (Hoebert et al. 2012) Hoebert et al. stellten in diesem Zusammenhang fest, dass verglichen mit 2008 zwar die Diagnosen ,Schlafstörung' oder ,Angststörung ${ }^{\star}$ seltener gestellt werden, dass aber auch der prozentuale Anteil an BZD-Verordnungen bei Patienten mit diesen Krankheitsbildern zurück ging und, dass Patienten öfter nur ein Rezept anstatt mehrere erhielten. Insgesamt kamen die Autoren zu dem Schluss, dass die geänderten Erstattungsregelungen der Krankenkassen zu einem moderaten Rückgang der BZD-Nutzung geführt hätten. Jedoch nahmen Patienten, die mehr als ein BZD-Rezept für Insomnie erhalten hatten, eine begonnene Therapie in 2009 länger ein als im Jahr zuvor. Dies wurde so interpretiert, dass möglicherweise nur die „Entschlossenen“ dazu bereit waren, dass Medikament langfristig selbst zu bezahlen, während weniger Entschlossene nur ein oder gar kein Rezept einlösten. Nur einen moderaten Effekt konnte auch eine US-amerikanische Studie zeigen, die sich mit der veränderten BZD-Nutzung nach Erstattungsausschluss durch die staatliche Versicherung Medicare beschäftigte (Chen und Kreling 2014). Auf der anderen Seite stellten Wagner et al. fest, dass der Verordnungsrückgang durch die veränderten Medicare-Vorgaben nicht zwangsweise zu einer Reduktion der mit einer BenzodiazepinEinnahme einhergehenden Komplikationen führt, bezogen sich dabei jedoch nur auf Hüftfakturen und nicht auf Unfälle im Allgemeinen, kognitive Einschränkungen sowie Suchtund Abhängigkeitsentwicklung (Wagner et al. 2007). Eine italienische Studie von 2001 kommt ebenfalls zu dem Ergebnis, dass eine Eigenfinanzierung der Medikamente die weitverbreitete Nutzung nicht stoppen konnte (Barbui et al. 2001). 
Des Weiteren ist zu überlegen, ob es sinnvoll wäre, pflanzliche und/oder freiverkäufliche Medikamente, wie Doxylamin, in begründeten Fällen auf Kassenrezept verordnen zu können. Dies ist z. Z. nicht möglich. Patienten müssen an dieser Stelle die „bessere Alternative" selbst bezahlen, während Benzodiazepine und Z-Substanzen im Prinzip verordnungsfähig sind. Das Privatrezept erscheint somit beinah als logisch, wenn Patienten dazu bewegt werden sollen, zunächst andere Wirkstoffgruppen zu nutzen.

\subsubsection{Sorgloser Umgang mit selbstbezahlten Medikamenten?}

Ein weiteres Problem der Privatverordnung von Benzodiazepinen und Z-Substanzen ist die veränderte Wahrnehmung der Medikamente durch Patienten. Der Unterschied zwischen einem rezeptpflichtigen Präparat, das von einem Arzt verschrieben werden muss, aber nicht von der gesetzlichen Krankenkasse bezahlt wird (blaues Privatrezept) und einem apothekenpflichtigen, aber freiverkäuflichen Mittel, das auch ohne vorherigen Arztkontakt erworben werden darf (grünes Rezept), ist möglicherweise vielen Patienten nicht klar (Ärzte Zeitung 2018)- zumal einige Praxen für beide Rezeptformen blaues Papier verwenden und die Kosten in beiden Fällen vom Patienten getragen werden. Ein grünes Rezept ist als Merkhilfe bzw. als Kaufempfehlung gedacht und wird häufig für Medikamente wie Kopfschmerztabletten oder Erkältungsmittel verwendet. Aber auch manche Schlaf- und Beruhigungsmittel, wie das Antihistaminikum Doxylamin, bekannt als Schlafsterne ${ }^{\circledR}$, sind so erhältlich. Insgesamt sind 50\% der in Apotheken abgegebenen Medikamente nicht rezeptpflichtig (Glaeske 2017). Wie die oben dargestellte Diskussion im Rahmen des Doktorandenkolloquiums vermuten lässt, könnten auf Privatrezept verschriebene Benzodiazepine und Z-Substanzen als ähnlich harmlos aufgefasst werden. Zwar konnte eine deutsche Online-Umfrage von 2013 zeigen, dass die Teilnehmer die Sicherheit von freiverkäuflichen Mitteln ähnlich einschätzen, wie die von verschreibungspflichtigen Medikamenten (Barrenberg und Garbe 2015). Dies könnte jedoch bedeuten, dass auch verschreibungspflichtige Medikamente für harmlos bzw. immer sicher gehalten werden oder eben freiverkäufliche Mittel für nicht immer harmlos. Allerdings hatten knapp 18\% der Teilnehmer die freiverkäuflichen Medikamente anders oder für einen anderen Zweck eingenommen. Und während fast die Hälfte der Befragten angab, immer den Beipackzettel zu lesen, lasen 6\% diesen nie. An Krankenhausaufnahmen auf Grund von Medikamentennebenwirkungen sind freiverkäufliche Mittel immerhin in 54\% der Fälle beteiligt (Schmiedl et al. 2014).

Zwar gibt es schon eine Reihe von Studien, die die Einstellung von Patienten zu Benzodiazepinen darstellen, aber wie die Metaanalyse von Sirdifield et al. zeigen konnte, stammen diese nicht aus Deutschland und befassen sich nicht mit dem Problem der HypnotikaPrivatrezepte für Kassenpatienten (Sirdifield et al. 2017). Daher wäre es sinnvoll, in einer patientenzentrierten Interviewstudie zu untersuchen, ob ein BenzoZ-Privatrezept wirklich die Wertigkeit des Medikaments verändert - sowohl in die eine als auch in die andere Richtung. Werden die Tabletten durch den finanziellen Aufwand zu „etwas Besonderem“ mit dem sparsam umgegangen werden muss, oder geschieht genau das Gegenteil? 


\subsection{Keine Orientierungshilfe zur Versorgung geerbter Patienten}

Zunächst einmal muss festgestellt werden, dass sich einzelne Auskünfte der Krankenkassen, Kassenärztlichen Vereinigungen, der örtlichen Apotheke und der niedersächsischen Apothekenkammer bezüglich der korrekten Verordnung von Schlaf- und Beruhigungsmitteln widersprechen. So verweist die Apothekenkammer bzgl. der Frage, ob Teilmengen, z. B. zwei Tabletten Diazepam $5 \mathrm{mg}$, verordnungsfähig sind, auf die Apothekenbetriebsordnung und die Tatsache, dass bei Auseinzelung nicht mehr die Arzneimittelpreisverordnung gilt. Der Apotheker darf eigenständig einen Preis berechnen, der dann aber wahrscheinlich in Widerspruch zu den Lieferverträgen mit den gesetzlichen Krankenkassen steht. Daher müsste bei einer GKV-Verordnung zunächst mit der jeweiligen Krankenkasse geklärt werden, wie viel zwei Tabletten Diazepam à $5 \mathrm{mg}$ kosten sollen bzw. dürfen. Im praktischen Alltag macht dies eine Verordnung von Teilmengen über ein Kassenrezept unmöglich, was sich mit der Wahrnehmung der Studienteilnehmer deckt. Dass ohne Rücksprache mit dem Arzt - wie von einer Göttinger Apotheke berichtet - auf die kleinste Packungsgröße aufgestockt und anstelle von zwei zehn Tabletten (N1) ausgegeben werden können, lässt sich weder aus der Apothekenbetriebsordnung (Bundesministerium der Justiz und für Verbraucherschutz 2017), noch dem aus dem in der Betriebsordnung erwähnten Fünften Sozialgesetzbuch und dem diesbezüglichen Rahmenvertrag (Spitzenverband Bund der Krankenkassen und Deutscher Apothekerverband 2016) entnehmen.

Ebenfalls bestehen in Hinblick auf das „Recht“ eines GKV-versicherten Patienten ein Kassenrezept zu erhalten, sehr unterschiedliche Ansichten. Während die KVN dies verneint, teilt die AOK mit, dass Patienten laut dem Bundesmantelvertrag für Ärzte sehr wohl ein Recht auf ein Kassenrezept haben. Nachdem unter 5.5.3 dargestellten Textes dieses Vertrages (Kassenärztliche Bundesvereinigung 2017) bleibt das Recht des Patienten auf ein Kassenrezept ein juristischer Graubereich, je nachdem, ob der Arzt das Präparat als „für die Behandlung (...) notwendig" erachtet oder nicht. Folglich ist die Verordnung von „Life-Style“-Medikamenten auf Privatrezept kein Muss, aber erlaubt. Anders verhält es sich bei medizinisch indizierten Mitteln. Dies deckt sich auch mit den von der AOK zugesendeten Handlungsempfehlungen der Ärztehammer Hamburg, der Kassenärztlichen Vereinigung Hamburg und der Apothekerkammer Hamburg, (Apothekenkammer Hamburg 2011), indem dort klar festgestellt wird: „Besteht bei gesetzlich Versicherten eine Indikation zur Gabe der Medikamente, ist ein Ausweichen auf Privatrezept nicht zulässig. “ Einige der dort erwähnten Hilfestellungen wurden bereits von mehreren Teilnehmern dieser Studie umgesetzt, wie beispielsweise das „Einholen einer zweiten Meinung eines in der Suchtmedizin erfahrenen Arztes oder eines Psychiaters“ beim Vorliegen einer schweren psychiatrischen Erkrankung, die eine langfristige Verordnung von Benzodiazepinen erforderlich machen.

Des Weiteren heißt es in dem Artikel, dass durch eine adäquate Aufklärung des Patienten sowie die Verwendung alternativer Therapien Langzeitverordnungen vermieden werden 
sollen (Apothekenkammer Hamburg 2011). Doch wie die Ergebnisse der Studie zeigen, ist es nicht die Entscheidung über eine eventuelle Neuverordnung, die größtenteils das Ausweichen auf ein Privatrezept bedingt; es sind die bereits Benzodiazepin- oder Z-Substanzabhängigen geerbten Patienten, die keine schwere psychiatrische Erkrankung haben. Hier bietet weder die Anlage III der Arzneimittelverordnung noch die Handlungsempfehlung aus Hamburg einen praxistauglichen Lösungsverschlag. Im Fall einer festgestellten Medikamentenabhängigkeit wird dort zu einer Überweisung an einen Suchtmediziner, einen Psychiater und/oder einer ambulanten Beratungsstelle oder einer Selbsthilfegruppe geraten. Die Weiterverordnung ist nicht vorgesehen und findet im Alltag doch statt, da ein Entzug aus verschiedenen Gründen vom Arzt und/oder Patient als nicht realisierbar angesehen wird oder schlichtweg kein Termin innerhalb der nächsten Monate zur Verfügung steht.

Von einer formal korrekten Klassifizierung dieser Patienten als „medikamentenabhängig“ rät laut telefonischer Auskunft sowohl die AOK ab als auch das Hamburger Ärzteblatt in einem Artikel zum Berufsrecht: „Die Unterhaltung einer Sucht mit dem Suchtstoff stellt bei der Benzodiazepinabhängigkeit grundsätzlich kein anerkanntes Behandlungskonzept dar.[...] Bei einer festgestellten Anhängigkeit wird vom Arzt ein Behandlungskonzept mit Entzugs- und Reduktionsplan und Kontrollen erwartet.“ Auch hier wird zu einer Überweisung an die oben genannt Stellen geraten (Kiehn 2009). Wie schnell oder wie langsam das Ausschleichen des Medikamentes gehen darf, wurde weder durch den Mitarbeiter der AOK noch im Artikel von Kiehn erwähnt. So erklärt sich eventuell auch die unter 5.2.7.2 dargestellte Annahme eines Teilnehmers, Benzodiazepine könnten beim sukzessiven Reduzieren nicht auf Kassenrezept verordnet werden. Möglicherweise ist das ein Grund dafür, dass die Diagnose Schlaf- und Beruhigungsmittelabhängigkeit selten gestellt wird. Wenn überhaupt, dann eher bei jungen Patienten mit Polytoxikomanie (Markota et al. 2016; American Psychiatric Association 2013). Auch wenn das ambulante Ausschleichen von Schlafund Beruhigungsmitteln theoretisch möglich ist, bleibt dies ein schwieriges Unterfangen insbesondere bei Langzeiteinnahme. Die telefonische Auskunft der AOKBeratungsapotheke ist diesbezüglich mehr als pessimistisch („Das ist so schwierig. Das können Sie im ambulanten Bereich vergessen"). In einer Interventionsstudie hatten 33\% der Patienten ihren Konsum nicht geändert, aber immerhin hatten 52\% diesen reduziert (Morrice und Iliffe 1992). Daher sollte auch bei älteren und multimorbiden Patienten eine Entwöhnung oder Dosisreduktion nicht von vornherein als Option ausgeschlossen werden. Das Argument einer QZ-Teilnehmerin, dass ein stationärer Aufenthalt in einem solchen Fall vermieden werden sollte, um die Gesamtsituation nicht durch beispielweise Infektionen mit multiresistenten Krankenhauskeimen oder Desorientierung auf Grund der ungewohnten Umgebung zu verschlechtern, ist jedoch ebenfalls gut nachvollziehbar.

Neben dem unter 5.5.3 erwähnten Artikeln von Haasen und Holzbach (Haasen und Holzbach 2009) sowie der Arbeit von Kiehn (Kiehn 2009) erschien in der gleichen Ausgabe des Hamburger Ärzteblattes ein Text, in dem es hieß: „Benzodiazepine und vergleichbare Wirkstoffe dürfen nur bei streng gestellter Indikation verordnet werden. Außerdem sollte 
eine gute, nachvollziehbare Dokumentation erstellt werden. Es drohen sonst Anträge der Krankenkassen auf Regresse, die nur schwer abzuwenden sind.“ (Voelker 2009) Das generell eine gute Dokumentation aller medizinischer Behandlungen sinnvoll ist, dürfte unstrittig sein. Jedoch könnten solche Hinweise dazu führen, dass das Privatrezept aus Angst vor einem Regress verstärkt genutzt wird. Auf der anderen Seite erscheint der Rat der BARMER Ersatzkasse, für jeden einzelnen Patienten einen Antrag auf Kostenübernahme zu stellen, der dann vom MDK überprüft wird, bürokratisch und sehr zeitaufwändig. Da mittlerweile gute Alternativen zur Behandlung von Schlaf- und Angststörungen zur Verfügung stehen, hätte eine Überprüfung wahrscheinlich ohnehin wenig Erfolg - es sei denn, der MDK erkennt an, dass der betroffene Patient nicht zu einem Entzug in der Lage ist.

Laut Auskunft der Beratungsapotheke der AOK sind Benzodiazepinen, die eine weitere oder andere Zulassung haben, z. B. als Anxiolytikum, von der Anlage III ausgenommen und dürften länger als vier Wochen verordnet werden. Dies ist aber nicht unmittelbar aus den beiden Abschnitten, die Benzodiazepine betreffen zu ersehen, wie Abbildung 12 zeigt.

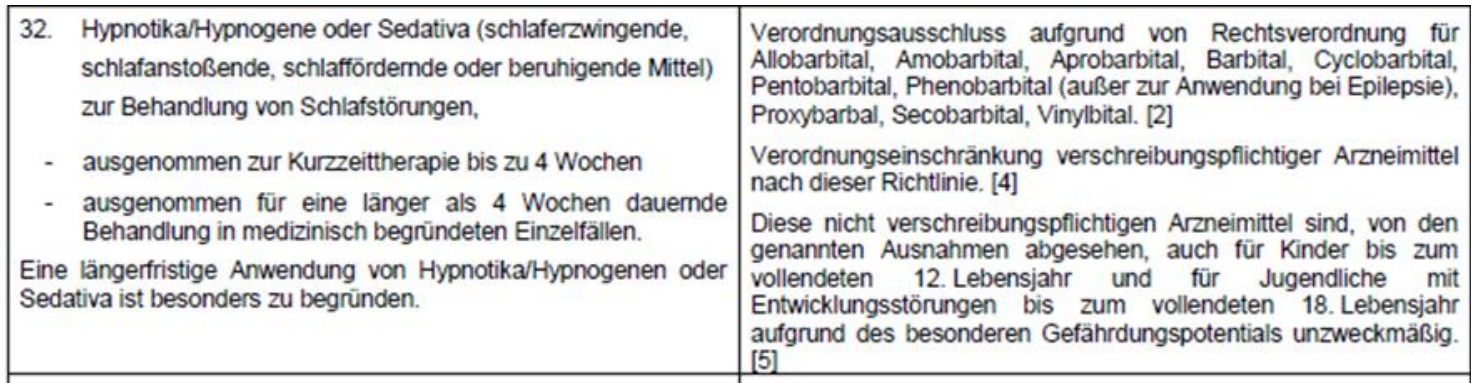

\begin{tabular}{|l|l|}
\hline 45. Tranquillantien, & $\begin{array}{l}\text { Verordnungsausschluss aufgrund von Rechtsverordnung für } \\
\text { Methaqualon. [2] } \\
\text { - ausgenommen zur Kurzzeittherapie bis zu 4 Wochen } \\
\text { - ausgenommen für eine länger als } 4 \text { Wochen dauernde } \\
\begin{array}{l}\text { Behandlung in medizinisch begründeten Einzelfällen. } \\
\text { Eine längerfristige Anwendung von Tranquillantien ist besonders } \\
\text { zu begründen. }\end{array}\end{array}$ \\
nach dieser Richtlinie. [4]
\end{tabular}

Abbildung 12: Auszug aus der Anlage III der Arzneimittelrichtlinie (Stand 02.02.2018)

Des Weiteren ist zu Zeit ist nicht klar definiert, ob auf mehrere Wochen bis Monate angelegte Entzugstherapien zu diesen, auch in Abschnitt 32 und 45 der Anlage III erwähnten, „Einzelfällen“ zu rechnen sind und wie sie dokumentiert werden sollen (Schulz 2014). 


\subsection{Schlussfolgerung}

Die Verordnung von Benzodiazepinen und Z-Substanzen bleibt ein schwieriges Thema, auch wenn sich das Bewusstsein für die Nebenwirkungen bei Ärzten und Patienten in den letzten Jahrzehenten geändert hat und somit immer weniger Schlaf- und Beruhigungsmittel verschrieben werden. Viele Hausärzte betreuen Patienten, die schon sehr lange ein solches Medikament einnehmen und bei denen womöglich von einer Einnahme bis ans Lebensende ausgegangen werden muss. Das Privatrezept bietet den Verschreibenden eine Möglichkeit mit der Ambivalenz zwischen Verordnen-wollen und Nicht-verordnen-wollen umzugehen. In der Regel wird bei Langzeitkonsum nicht der Entzug als Ziel gesehen, sondern die Limitierung der konsumierten Menge (Dosis und Frequenz) auf ein möglichst geringes Maß. Die Verordnung auf einem Privatrezept soll durch die finanzielle Belastung dem Patienten als Anreiz dienen, dieses Ziel zu erreichen. Allerdings wird dabei außer Acht gelassen, dass Benzodiazepine und Z-Substanzen preiswerte Medikamente sind und, dass bei einer Abhängigkeit die Kosten der Droge keine Rolle spielen. Auch wenn der Vergleich zum Doping (Singler und Treutlein 2013) nur bedingt gezogen werden kann, ist er treffender als der von der BARMER geäußerte Vergleich mit Herzmedikamenten, da letztere in der Regel weder eine physische noch psychische Abhängigkeit verursachen.

$\mathrm{Ob}$ die Handlungsstrategie Privatrezept zur Konsumreduktion erfolgreich ist, wird oft nicht hinterfragt. Hier bedarf es weiterer Studien (Patienteninterviews, Vergleich der Einnahmedaten von Patienten mit und ohne GKV-Rezept). Auch wenn es schon einzelne Arbeiten und Review-Artikel zur Patientenwahrnehmung von Benzodiazepinen und ZSubstanzen gibt (Filardi et al. 2017), ist die Patientenperspektive des Privatrezeptes bisher unerforscht.

Ein weiteres Problem ist die formal richtige Verordnungsweise bei Langzeitkonsum. Die von den Krankenkassen und Kassenärztlichen Vereinigungen zitierte Anlage III der Arzneimittelrichtlinie bietet keine Orientierungshilfe für die Betreuung von bereits abhängigen Patienten. Hier bedarf es klarer und im praktischen Alltag umsetzbarer Vorgaben, die einheitlich kommuniziert werden müssten. Letztendlich böte dies auch den Verordnenden Sicherheit und würde möglichweise dem Wunsch nach einer mittels Privatrezept erreichten Hemmschwelle entgegenwirken. 


\section{$7 \quad$ Zusammenfassung}

Hintergrund: Die Anzahl der Schlaf- und Beruhigungsmittelverordnungen im ambulanten Bereich zu Lasten der Krankenkassen ist in den letzten Jahrzehnten deutlich zurückgegangen. Jedoch werden Benzodiazepine und Z-Substanzen (BenzoZ) häufig auf Privatrezept verschrieben - auch für kassenärztlich versicherte Patienten. Genaue quantitative Angaben diesbezüglich existieren nicht. Die Gruppe der Allgemeinmediziner und hausärztlich tätigen Internisten verordnet im Vergleich zu anderen Fachrichtungen die meisten dieser Medikamente.

Fragestellung: Warum stellen Hausärzte kassenärztlich versicherten Patienten Privatrezepte für Schlaf-und Beruhigungsmittel aus? Welche Faktoren beeinflussen den Entscheidungsprozess? Ziel dieser Arbeit ist das Erstellen einer Theorie, die dieses Verhalten erklärt und rahmt.

Methode: Insgesamt 17 Hausärzte/-ärztinnen aus der ländlichen Region des Eichsfelds (südöstliches Niedersachsen/ nord-westliches Thüringen) und der Großstadt Hannover wurden leitfadengestützt interviewt. Die qualitative Auswertung der Daten erfolgte nach Grounded Theory mit Hilfe von MAXQDA. Zusätzlich wurde eine Gruppendiskussion im Rahmen eines Qualitätszirkels mit 5 Hausärztinnen ausgewertet. Telefonisch erfolgte eine Datenrecherche der Kassenärztlichen Vereinigungs- (KV) und Krankenkassenvorgaben sowie der Vorgaben einer örtlichen Apotheke und der Apothekenkammer zu BenzoZVerordnungen.

\section{Ergebnisse:}

In einem übergeordneten paradigmatischen Modell zeigt sich Ambivalenz als zentrales Phänomen. Hausärzte nehmen BenzoZ ambivalent wahr, da solche Medikamente auf der einen Seite als hilfreich und wirkungsvoll betrachtet, aber auf der anderen Seite auf Grund der Nebenwirkungen gemieden werden sollen. Das Ausstellen eines Privatrezeptes ist eine Handlungsstrategie, um beiden Empfindungen gerecht zu werden. Betrachtet man diese Handlungsstrategie im Detail, zeigt sich ein zweites paradigmatisches Modell, dessen zentrales Phänomen das Kreieren einer Hemmschwelle ist. Hierzu wird angenommen, dass Menschen mit einem Medikament sparsamer umgehen, wenn sie einen höheren Eigenanteil bezahlen müssen. Hausärzte nutzen das Privatrezept vorwiegend als Instrument, um den BenzoZ-Konsum ihrer Patienten einzuschränken. Ursächlich für den Wunsch nach einer Hemmschwelle zeigt sich u. a. die Unsicherheit der Ärzte darüber, wann und wie lange diese Medikamente von den gesetzlichen Krankenkassen bezahlt werden. So dass - obwohl BenzoZ als preiswert eingestuft werden - das Privatrezept auch gewählt wird, um eine Regressforderung zu vermeiden. Es dient also nicht nur dem Schutz der Patienten, sondern nicht zuletzt auch dem Selbstschutz der Ärzte. Die Anlage III der Arzneimittelrichtlinie 
(AMRL) des Gemeinsamen Bundesausschusses bietet bezüglich der Betreuung von bereits BenzoZ-abhängigen Patienten für die ein Entzug nicht realisierbar ist, keine Orientierungshilfe. Weiteren Einfluss auf die Wahl der Rezeptform - wenn auch einen geringeren - haben u. a. die Diagnose, die erwartete Problemdauer und der wahrgenommene Patiententyp.

\section{Diskussion:}

Ob Patienten durch den überschaubaren finanziellen Aufwand eines Privatrezeptes dazu bewegt werden, weniger Schlaf- und Beruhigungsmittel einzunehmen ist fraglich - insbesondere bei schon bestehender Abhängigkeit. Auch könnte bei medizinischen Laien der Eindruck entstehen, BenzoZ wären ähnlich nebenwirkungsarm wie freiverkäufliche Medikamente. Es fehlen Studien, die das Thema Privatrezept aus Patientensicht darstellen. Die ungenauen und teilweise widersprüchlichen Auskünfte der örtlichen Apotheke, der Apothekenkammer, der KV und der Krankenkassen sowie die Anlage III der AMRL bieten keine Hilfestellung im Praxisalltag. Eine klare und einheitliche Stellungnahme könnte helfen, den Anteil an Privatrezepten zu reduzieren. 


\section{Anhang}

\subsection{Artikelliste zu 2.5: Eistellung von Ärzten gegenüber Hypnotika}

\section{Pubmed:}

1) Albretsen CS (1991): Effekt av tilbakemelding og selvevaluering på forskrivning av beroligende mid-ler. Holdningen hos leger ved forskrivning av benzodiazepiner: Effect of feedback and self-evaluation on prescription of addictive agents. Position of physicians about prescribing bensodiaze-pines. Tidsskr Nor Laegeforen 111, 2775

2) Anthierens S, Pasteels I, Habraken H, Steinberg P, Declercq T, Christiaens T (2010): Barriers to nonpharmacologic treatments for stress, anxiety, and insomnia: family physicians' attitudes toward benzodiazepine prescribing. Can Fam Physician 뜨, 406

3) Bachmann CS, Berg EA, Spigset O, Slørdal L (2008): Benzodiazepinliknende hypnotika-holdninger og forskrivningspraksis blant allmennleger: Benzodiazepine-like hypnotics-attitudes and prescription practice among general practitioners. Tidsskr Nor Laegeforen $\underline{128}, 166-170$

4) Chambers CD, White OZ, Lindquist JH (1983): Physician attitudes and prescribing practices: a focus on minor tranquilizers. J Psychoactive Drugs $\underline{15}$, 55-59

5) Cook JM, Marshall R, Masci C, Coyne JC (2007): Physicians' perspectives on prescribing benzodiaz-epines for older adults: a qualitative study. J Gen Intern Med 22, 303-307

6) Deans HG, Skinner P (1992): Doctors' views on anxiety management in general practice. J R Soc Med $\underline{85}, 83-86$

7) Dièye AM, Sylla M, Ndiaye A, Ndiaye M, Sy GY, Faye B (2006): Benzodiazepines prescription in Dakar: a study about prescribing habits and knowledge in general practitioners, neurologists and psychiatrists. Fundam Clin Pharmacol 20, 235-238

8) Everitt H, McDermott L, Leydon G, Yules H, Baldwin D, Little P (2014): GPs' management strategies for patients with insomnia: A survey and qualitative interview study. $\mathrm{Br} \mathrm{J}$ Gen Pract $\underline{64}$, e112-e119

9) Flick U, Garms-Homolova V, Rohnsch G (2012): "And mostly they have a need for sleeping pills": physicians' views on treatment of sleep disorders with drugs in nursing homes. J Aging Stud 26, 484-494

10) Hamilton IJ, Reay LM, Sullivan FM (1990): A survey of general practitioners' attitudes to benzodiazepine overprescribing. Health Bull (Edinb) 4ㅇ, 299-303

11) Haw C, Stubbs J (2007): Benzodiazepines--a necessary evil? A survey of prescribing at a specialist UK psychiatric hospital. J Psychopharmacol (Oxford) 21, 645-649

12) Heiman EM, Wood G (1981): Patient characteristics and clinician attitudes influencing the prescribing of benzodiazepines. J Clin Psychiatry $\underline{42}, 71-73$

13) Hoffmann F (2013a): Benefits and risks of benzodiazepines and Z-drugs: comparison of perceptions of GPs and community pharmacists in Germany. Ger Med Sci 11, 1-7

14) Hoffmann F (2013b): Perceptions of German GPs on benefits and risks of benzodiazepines and Z-drugs. Swiss Med Wkly 143, w13745

15) Hoffmann F, Schmiemann G, Windt R (2014): Privat statt Kasse?: Einstellungen von Hausärzten und Apothekern zur Verordnung von Hypnotika. Dtsch Med Wochenschr $\underline{139}, 1153-1158$ 
16) Iliffe S, Curran HV, Collins R, Yuen Kee SC, Fletcher S, Woods B (2004): Attitudes to long-term use of benzodiazepine hypnotics by older people in general practice: findings from interviews with service users and providers. Aging Ment Health $\underline{8}, 242-248$

17) Johnson C, Baxter B, Brough R, Buchanan J (2007): Benzodiazepine prescribing: Lessons from interprofessional dialogue. Aust Fam Physician $\underline{36}$, 245-246

18) Kristensen $P$, Fjermestad $T$ (1991): Benzodiazepiner--holdninger og forskrivningspraksis blant all-mennpraktikere og psykiatere: Benzodiazepines--attitudes and prescription practice among general practitioners and psychiatrists. Tidsskr Nor Laegeforen 111, 2241-2245

19) Rogers A, Pilgrim D, Brennan S, Sulaiman I, Watson G, Chew-Graham C (2007): Prescribing ben-zodiazepines in general practice: a new view of an old problem. Health (London) $\underline{11}, 181-198$

20) Sällström G, Maasing R (1980): Swedish company doctors' attitudes to benzodiazepines and neuro-leptics used as minor tranquillizers. Pharmatherapeutica $\underline{2}, 380-386$

21) Sirdifield C, Anthierens S, Creupelandt H, Chipchase SY, Christiaens T, Siriwardena A (2013): General practitioners' experiences and perceptions of benzodiazepine prescribing: Systematic review and meta-synthesis. BMC Fam Pract 14, 191

22) Siriwardena AN, Apekey T, Tilling M, Dyas JV, Middleton H, Ørner R (2010): General practitioners' preferences for managing insomnia and opportunities for reducing hypnotic prescribing. J Eval Clin Pract 16, 731-737

23) Siriwardena AN, Qureshi Z, Gibson S, Collier S, Latham M (2006): GPs' attitudes to benzodiazepine and 'Z-drug' prescribing: a barrier to implementation of evidence and guidance on hypnotics. Br J Gen Pract $\underline{56}$, 964-967

24) Srisurapanont M, Garner P, Critchley J, Wongpakaran N (2005): Benzodiazepine prescribing behav-iour and attitudes: a survey among general practitioners practicing in northern Thailand. BMC Fam Pract $\underline{6}, 27$

25) Troest A (1993): Ordination af benzodiazepinpraeparater i Nykøbing Falster Kommune. En recep-tundersøgelse med henblik på ordinationsvaner i almen praksis: Prescriptions of benzodiazepines in the municipality of Nykøbing Falster. A study of prescriptions with reference to prescription habits in general practice. Ugeskr Laeg 155, 2029-2032

26) Verger P, Aulagnier M, Protopopescu C, Villani P, Gourrheux J-C, Bouvenot G, Paraponaris A (2004): Hypnotic and tranquillizer use among general practitioners in south-eastern France and its relation to occupational characteristics and prescribing habits. Fundam Clin Pharmacol $\underline{18}, 379-385$

27) Wolde GB, Dijkstra A, van Empelen P, Knuistingh Neven A, Zitman FG (2008): Psychological determinants of the intention to educate patients about benzodiazepines. Pharm World Sci 30, 336-342

\section{EMBASE:}

28) Boixet M, Batlle E, Bolibar I (1996): Benzodiazepines in primary health care: a survey of general practitioners prescribing patterns. Addiction $21,549-556$

29) Bjørner T, Kjølsrød L (2002): How GPs understand patients' stories. Eur J Gen Pract $\underline{8}$, 25-30

30) Bjørner T, Laerum E (2003): Factors associated with high prescribing of benzodiazepines and minor opiates. A survey among general practitioners in Norway. Scand J Prim Health Care 21, 115-120

31) Bourgeois J, Elseviers MM, Azermai M, van Bortel L, Petrovic M, Vander Stichele RR (2014): Barriers to discontinuation of chronic benzodiazepine use in nursing home residents: Perceptions of general practitioners and nurses. Eur Geriatr Med $\underline{5}, 181-187$ 
32) Cheung JMY, Atternäs K, Melchior M, Marshall NS, Fois RA, Saini B (2014): Primary health care practitioner perspectives on the management of insomnia: A pilot study. Aust $\mathrm{J}$ Prim Health $\underline{20}, 103$

33) Davidson W, Molloy W, Somers G, Bédard M (1994): Relation between physician characteristics and prescribing for elderly people in New Brunswick. CMAJ 150, 917-921

34) Dybwad TB, Kjolsrod L, Eskerud J, Laerum E (1997): Why are some doctors highprescribers of benzodiazepines and minor opiates? A qualitative study of GPs in Norway. Fam Pract 14, 361-368

35) Holden JD, Hughes IM, Tree A (1996): Attitudes of general practitioners to benzodiazepine prescribing and withdrawal. J Ment Health $\underline{5}, 501-506$

36) Monette J, Tamblyn RM, McLeod PJ, Gayton DC (1997): Characteristics of physicians who frequently prescribe long-acting benzodiazepines for the elderly. Eval Health Prof $\underline{20}$, 115-130

37) Subelj M, Vidmar G, Svab V (2010): Prescription of benzodiazepines in Slovenian family medicine: a qualitative study. Wien Klin Wochenschr 122, 474-478

38) Zisselman MH, Rovner BW, Yuen EJ, Sholevar D (1997): Physician rationale for benzodiazepine prescriptions to elderly hospitalized patients. Am J Geriatr Psychiatry $\underline{5}, 167-171$

\section{Keine neuen oder relevanten Artikel über CC MED.}




\title{
8.2 Informationsblatt zur Studie
}

\section{UNIVESTTITISMEIIIN :
COTIINGEN : UMG}

Universitätsmedizin Göttingen, 37099 Göttingen

Direktorin des Instituts für Allgemeinmedizin

Institut für Allgemeinmedizin

Direktorin

Prof. Dr. Eva Hummers-Pradier, Humboldtallee 38

37099 Göttingen Briefpost Humboldtallee 38, 37073 Göttingen Adresse 0551 / 39-22638 Telefon 0551 / 39-9530 Fax

\section{Informationstext zur wissenschaftlichen Studie}

\author{
„Hypnotika in der Praxis“"
}

\section{Worum geht es?}

In dieser Studie geht es um die Verordnung und Einnahme von Hypnotika und Sedativa, insbesondere Benzodiazepine und Z-Substanzen. Aus der Literatur wissen wir, dass es hier immer wieder zu Problemen kommt. Stichwortartig seien nur „Wunschverordnungen“ und „Dauerverordnungen" genannt. Allerdings wissen wir nur wenig über die ärztliche Perspektive, also welche Erfahrungen z. B. Hausärzte[innen] bei der Verordnung dieser Medikamente machen oder wie sie sich praktisch zwischen Lehrbuchwissen und Praxisalltag bewegen. Hierüber mehr zu erfahren, ist Ziel dieser Studie.

Wie ist der Ablauf der Studie?

Wir sind sehr an Ihrer Meinung und Ihren Erfahrungen interessiert und würden gerne ein sog. offenes Interview mit Ihnen führen. Das, was Ihnen zu diesem Thema wichtig ist, steht dabei im Vordergrund. Das Treffen soll in entspannter Atmosphäre an einem Ort Ihrer Wahl stattfinden, z. B. in Ihrer Praxis oder bei Ihnen zu Haus. Erfahrungsgemäß ist die Dauer der Interviews recht unterschiedlich. Manchmal dauert es ca. 30 Minuten, manchmal auch länger.

Sie erhalten eine Aufwandsentschädigung von 50 Euro. 
Was geschieht mit den Daten?

Das Interview wird auf Tonband aufzeichnet.

Nach dem Interview und vor Beginn der Auswertung erhalten Sie eine Abschrift Ihres Interviews („Transkript“) zur Durchsicht mit Streichungs- und Anonymisierungsmöglichkeit, so dass keine Rückschlüsse auf Ihre Person erfolgen können. Die anschließende Auswertung der Transkripte erfolgt ausschließlich in anonymisierter Form durch Projektpersonal bzw. Mitarbeiter des Instituts für Allgemeinmedizin. Die Transkripte werden nicht an Dritte weitergegeben oder für andere Zwecke oder Projekte verwendet.

Transkripte und Audiodaten werden anonymisiert unter den Bedingungen des Datenschutzes bzw. der guten wissenschaftlichen Praxis für einen Zeitraum von 10 Jahren gespeichert. Die personenbezogenen Audiodateien und nicht anonymisierten Abschriften werden unter Verschluss gehalten; sie sind nur dem unmittelbar zuständigen Projektmitarbeiter und der Projektleiterin in der UMG zugänglich und werden nach Abschluss der Studie gelöscht.

Was sollten Sie noch wissen?

Die Teilnahme an der Studie ist freiwillig. Sie können jederzeit und ohne Angabe von Gründen diese Zustimmung widerrufen, ohne dass Ihnen dadurch Nachteile entstehen. Alle bis dahin erhobenen Daten sowie die Audioaufnahmen im Original und die Sicherheitskopie würden in diesem Fall unverzüglich vernichtet werden.

Für weitere Fragen stehen wir natürlich zur Verfügung. Am besten erreichen Sie uns unter der folgenden Handynummer: 0170-1880067 oder unter der unten stehenden E-Mail Adresse.

Katharina Schmalstieg katharina.schmalstieg@med.uni-goettingen.de
Prof. Dr. med. Eva Hummers-Pradier (Direktorin des Instituts) 


\subsection{Einverständniserklärung Einzelinterviews}

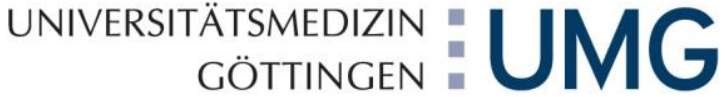

Universitätsmedizin Göttingen, 37099 Göttingen

Direktorin des Instituts für Allgemeinmedizin

Institut für Allgemeinmedizin

Direktorin

Prof. Dr. Eva Hummers-Pradier, Humboldtallee 38

37099 Göttingen Briefpost Humboldtallee 38, 37073 Göttingen Adresse 0551 / 39-22638 Telefon 0551 / 39-9530 Fax eva.hummers-pradier@med.uni-goettingen.de E-Mail

18. September 2019 Datum

\section{Einverständniserklärung zur Teilnahme an einer wissenschaftlichen Studie}

Teilnehmer(in): Vor- u. Nachname (in Druckbuchstaben)

Ich wurde über die o. g. Studie „Hypnotika in der Praxis“ vollständig aufgeklärt und habe den Informationstext gelesen und verstanden. Ich hatte die Gelegenheit, Fragen dazu zu stellen. Die Antworten habe ich verstanden und akzeptiert. Ich hatte genügend Zeit, mich zur Teilnahme zu entscheiden. Ich weiß, dass die Teilnahme freiwillig und mit keinem unmittelbaren persönlichen Nutzen oder Nachteilen für mich verbunden ist.

Ich weiß, dass ich jederzeit und ohne Angabe von Gründen diese Zustimmung widerrufen kann, ohne dass dadurch Nachteile für mich entstehen. Alle bis dahin erhobenen Daten sowie die Audioaufnahmen im Original und die Sicherheitskopie würden in diesem Fall unverzüglich vernichtet werden. Ich erhalte vor Beginn der Auswertung eine Abschrift meines Interviews („Transkript") zur Durchsicht mit Streichungs- und Anonymisierungsmöglichkeit, so dass keine Rückschlüsse auf meine Person erfolgen können. Ich bin damit einverstanden, dass das Interview auf Tonband aufgezeichnet wird. Ich bin damit einverstanden, dass die im Rahmen der Studie erhobenen Daten und Audiodaten anonymisiert unter den Bedingungen des Datenschutzes bzw. der guten wissenschaftlichen Praxis für einen Zeitraum von 10 Jahren gespeichert werden. Die personenbezogenen Audiodateien und nicht anonymisierten Abschriften werden unter Verschluss gehalten; sie sind nur dem unmittelbar zuständigen Projektmitarbeiter und der Projektleiterin in der UMG zugänglich und werden nach Abschluss der Studie gelöscht. Die Interviewabschriften werden in anonymisierter Form von Projektpersonal bzw. Mitarbeitern des Instituts für Allgemeinmedizin ausgewertet, Sie werden nicht an Dritte weitergegeben oder für andere Zwecke oder Projekte verwendet.

Ich habe den Informationstext und eine Kopie dieser Einwilligung erhalten.

Ort, Datum,

Teilnehmer(in): Unterschrift
Ort, Datum,

Unterschrift Studienmitarbeiter(in) 


\subsection{Einverständniserklärung Qualitätszirkel}

\section{UNIVERSITÄTSMEDIZIN :
GÖTTINGEN : $\mathbf{M G}$}

Universitätsmedizin Göttingen, 37099 Göttingen Direktorin des Instituts für Allgemeinmedizin

Prof. Dr. Eva Hummers-Pradier, Humboldtallee 38
Institut für Allgemeinmedizin

Direktorin

Prof. Dr. E. Hummers-Pradier

37099 Göttingen Briefpost Humboldtallee 38, 37073 Göttingen Adresse 0551 / 39-22638 Telefon 0551 / 39-9530 Fax eva.hummers-pradier@med.uni-goettingen.de E-Mail

18. September 2019 Datum

\section{Einverständniserklärung zur Teilnahme an einer wissenschaftlichen Studie}

Teilnehmer(in): Vor- u. Nachname (in Druckbuchstaben)

Ich wurde über die o. g. Studie „Hypnotika in der Praxis“ vollständig aufgeklärt und hatte die Gelegenheit, Fragen dazu zu stellen. Die Antworten habe ich verstanden und akzeptiert. Ich hatte genügend Zeit, mich zur Teilnahme zu entscheiden. Ich weiß, dass die Teilnahme freiwillig und mit keinem unmittelbaren persönlichen Nutzen oder Nachteilen für mich verbunden ist.

Ich weiß, dass ich jederzeit und ohne Angabe von Gründen diese Zustimmung widerrufen kann, ohne dass dadurch Nachteile für mich entstehen. Ich bin damit einverstanden, dass das Gespräch auf Tonband aufgezeichnet und zum Zweck der Auswertung eine Abschrift (Transkript) angefertigt wird. Im Falle meines Widerrufs werden in der Abschrift meine Beiträge gelöscht sowie die Audiodateien unverzüglich vernichtet. Ich bin damit einverstanden, dass die im Rahmen der Studie erhobenen Daten und Audiodaten anonymisiert unter den Bedingungen des Datenschutzes bzw. der guten wissenschaftlichen Praxis für einen Zeitraum von 10 Jahren gespeichert werden. Die personenbezogenen Audiodaten und die nicht anonymisierte Abschrift werden unter Verschluss gehalten; sie sind nur dem unmittelbar zuständigen Projektmitarbeiter und der Projektleiterin in der UMG zugänglich und werden nach Abschluss der Studie gelöscht. Die Abschrift wird in anonymisierter Form von Projektpersonal bzw. Mitarbeitern des Instituts für Allgemeinmedizin ausgewertet. Durch die Anonymisierung werden keine Rückschlüsse auf meine Person erfolgen können. Die Daten werden nicht an Dritte weitergegeben oder für andere Zwecke oder Projekte verwendet.

Ich habe eine Kopie dieser Einwilligung erhalten.

Ort, Datum,

Teilnehmer(in): Unterschrift
Ort, Datum,

Unterschrift Studienmitarbeiter(in) 


\subsection{Interviewleitfaden}

Vorstellung (Zusammenfassung des Informationstextes)

1.) des Interviewers:

- Name, Beruf, momentaner Arbeitsplatz (Promotion am Institut für Allgemeinmedizin Göttingen)

2.) der Studie:

- Interviews, um die Erfahrungen von Ärzten zu erfassen

- Freiwillige Teilnahme/Widerruf jederzeit möglich

- Tonbandaufzeichnung des Interviews

- Anfertigung eines Transkripts/anonyme Weiterverwertung der Daten

- Jeder Teilnehmer erhält eine Kopie seines Transkripts und kann bei Bedarf Passagen streichen

- Schriftliche Einverständniserklärung

\section{Einleitung}

Wenn ich Ihnen gleich Fragen stelle, haben Sie so viel Zeit, wie Sie brauchen, um diese in Ruhe zu beantworten. Ich werde Ihnen zu Beginn zunächst nur zuhören und mir als Gedächtnishilfe Stichpunkte notieren, auf die ich dann später nochmal zurückkommen will.

\section{Erzählaufforderung Schlafmittel}

Wenn Sie jetzt einmal an die Verordnung von Benzodiazepinen und Z-Substanzen, wie Zolpidem und Zopiclon denken: Was fällt Ihnen spontan dazu ein? Vielleicht erinnern Sie sich in diesem Zusammenhang auch an den einen oder anderen Patientenkontakt. Erzählen Sie doch mal.

Nachfragen (durch Detaillierungsaufforderungen oder Paraphrasieren), z. B.:

- Ich würde gerne nochmal auf die Notizen, die ich mir gemacht habe, zu sprechen kommen.

- Sie erwähnten, dass... (X) Können Sie das genauer erläutern?

- In dem von Ihnen geschilderten Fall ergab sich also der Umstand/ das Problem/ die Situation, dass...(X). Wie sind Sie damit umgegangen?

\section{Verwendete Schlafmittel}

- Welche Erfahrungen haben Sie mit bestimmen Benzodiazepinen und Z-Substanzen gemacht?

- Warum haben Sie sich für die Verwendung dieser Präparate entschlossen? 
- Welche unerwünschten Auswirkungen haben Sie bei der Verordnung dieser Medikamente erlebt, oder welche befürchten Sie? (Stürze, Demenz??)

- Es wird ja manchmal berichtet, dass Z-Substanzen viel besser seien als Benzodiazepine. Was sind Ihre Erfahrungen diesbezüglich?

- Welche Erfahrungen haben Sie mit Alternativen zu Benzodiazepinen und ZSubstanzen gemacht?

\section{Erzählaufforderung Privatrezepte}

In letzter Zeit werden Schlaf- oder Beruhigungsmittel oft auf Privatrezepte verschrieben. Viel-leicht kennen Sie diese aus Ihrem eigenen Praxisalltag oder von Kollegen. Erzählen Sie mir doch von Ihren Erfahrungen.

oder wenn bereits zuvor erwähnt:

Sie erwähnten die Verordnung mittels Privatrezept. Aus welchem Grund haben Sie sich hier-zu entschlossen? Bitte versuchen Sie sich an die letzten Patientenkontakte zu erinnern und diese zu beschreiben.

- Gab es andere Fälle in denen Sie ein Privatrezept für einen kassenärztlich versicherten Patienten ausgestellt haben?

hier erneut „Nachfragen“ im obigen Sinne

\section{Einfluss Patient}

- Welche Rollen spielen Patienten dabei?

- Gibt es bestimmte Patientengruppen oder Patienten mit bestimmten Diagnosen, denen Sie häufiger ein Privatrezept ausstellen als anderen?

- Was wäre für Sie ein Grund zu sagen: „Nein, ich stelle Ihnen kein Rezept aus?“

- Erhalten Patienten ausschließlich eine Rezeptform (Kasse oder Privat) oder wechseln Sie auch mal zwischen den Verordnungsformen? Warum?

\section{Einfluss Mitbetreuer}

- Einige Ihrer Patienten, die Benzodiazepine und Z-Substanzen einnehmen, werden vielleicht gleichzeitig durch einen Psychologen oder Fachspezialisten betreut. Wie ist da Ihre Erfahrung?

\section{Einfluss Krankenkassen}

- Wie sehen Sie die Rolle der Krankenkassen in Bezug auf Benzodiazepinen und ZSubstanzen?

- Gab es schon mal Feedback oder Nachfragen? 


\section{Einfluss Arzneimittel-Richtlinie}

- Wie empfinden Sie die Vorgaben der aktuellen Arzneimittel-Richtlinie? (ggf. hier Stichworte: Richtlinie des Gemeinsamen Bundesausschusses, nur Kurzzeittherapie bis zu 4 Wochen, längere Therapie im Einzelfall begründen - falls der Eindruck entsteht, die Frage wird ungern/ausweichend beantwortet auf Grund der Unkenntnis aktueller Empfehlungen)

- Wenn Sie in Bezug auf die Verordnungsvorgaben von Benzodiazepinen und ZSubstanzen etwas ändern könnten, was würden Sie ändern?

\section{Notdienst}

- Wie erleben Sie die Verordnung von Schlaf- und Beruhigungsmittel im Notdienst?

\section{Computer}

- Abhängig vom verwendeten Computersystem erscheint manchmal ein Warnhinweis bei der Verordnung von Schlaf- und Beruhigungsmittel. Vielleicht kennen Sie das aus der eigenen Praxis. Wie gehen Sie damit um?

\section{Persönliche Erfahrung}

- Das Thema Schlaf ist ja etwas, das uns alle betrifft. Haben Sie denn schon mal persönliche Erfahrungen mit Schlaf- und Beruhigungsmitteln gemacht?

\section{Überleitung}

- Bevor ich Ihnen noch einige abschließende Fragen stelle: Ist Ihnen im Zusammenhang mit der Verordnung von Benzodiazepinen und Z-Substanzen noch etwas eingefallen, das wir noch nicht angesprochen haben?

\section{Demografische Informationen}

- Wie lange sind Sie schon Facharzt? Seit wann sind Sie niedergelassen? (Nur fragen, wenn dies nicht bereits auf der Homepage steht.)

- Was würden Sie schätzen, wie viele Patienten betreut Ihre Praxis insgesamt/betreuen Sie als einzelner Arzt (bei Praxisgemeinschaften)?

- Wieviel Zeit haben Sie im Schnitt für ein Patientengespräch?

- Wie würden Sie ihre Patientenpopulation beschreiben? Gibt es Besonderheiten im Hinblick auf die Patientenpopulation Ihrer Praxis (z. B. Migrantenanteil, Anteil an älteren Patienten, etc.)?

- Wie schätzen Sie die Verfügbarkeit und die Zusammenarbeit mit anderen Fachrichtungen, wie Psychiater oder Psychologen, im Einzugsgebiet Ihrer Praxis ein? 


\subsection{Transkriptionsregeln}

Jaaaaaaa Dehnung - je mehr Vokale aneinandergereiht werden, desto länger ist die Dehnung

viellei- $\quad$ Abbruch mitten im Wort

((lachend)) Kommentar der Transkribierenden zu nicht verbalen Ereignissen Dauer einer Pause in Sekunden

(US: doch) Unsicherheit in der Transkription

( ) Äußerung unverständlich - Länge der Klammer je nach Länge der unverständlichen Passage

nein betont

1.) Das Interview wortwörtlich transkribieren.

2.) Inhaltliche Fehler nicht korrigieren, z. B. „Es kann nicht jeder eine Konifere auf seinem Gebiet sein.“ Gemeint war natürlich Koryphäe.

3.) Die Ähms, Ähs und Hms mit Kommata im Redefluss abtrennen, also: „Dann habe ich, ähm, als ich, äh,... “ Bei häufig verwendeten Ähms oder der Gleichen ist es nicht entscheidend die absolut genaue Anzahl der Ähms zu erfassen.

4.) Unterbrechungen: Wenn derjenige sich selbst unterbricht, also einen Satz abbricht, dann kommt ein Bindestrich direkt an ihr/sein letztes Wort (s. oben).Wenn er/sie unterbrochen wird, dann wird in Klammern dahinter geschrieben ((wird unterbrochen)). 


\subsection{COREQ-Checkliste zur Darstellung qualitativer Forschungsergebnisse}

Wie die COREQ-Checkliste (Consolidated criteria for reporting qualitative studies (COREQ): 32-item checklist) als validiertes Instrument (Tong et al. 2007) zeigt, wurden alle relevanten Punkte zur Darstellung qualitativer Forschungsergebnisse beachtet. Da keine deutsche Version zur Verfügung stand, wurde das englische Original verwendet. Die Seitenangaben beziehen sich auf die vorliegende Arbeit.

\begin{tabular}{|c|c|c|}
\hline No. Item & Guide questions/description & $\begin{array}{l}\text { Reported on Page } \\
\#\end{array}$ \\
\hline \multicolumn{3}{|l|}{$\begin{array}{l}\text { Domain 1: Research team } \\
\text { and reflexivity }\end{array}$} \\
\hline \multicolumn{3}{|l|}{ Personal Characteristics } \\
\hline 1. Interviewer/facilitator & $\begin{array}{l}\text { Which author/s conducted the interview or fo- } \\
\text { cus group? }\end{array}$ & \#24 (4.3.3) \\
\hline 2. Credentials & $\begin{array}{l}\text { What were the researcher's credentials? E.g. } \\
\mathrm{PhD}, \mathrm{MD}\end{array}$ & \#119 (CV) \\
\hline 3. Occupation & $\begin{array}{l}\text { What was their occupation at the time of the } \\
\text { study? }\end{array}$ & \#24 (4.3.3) \\
\hline 4. Gender & Was the researcher male or female? & Female (s. first name) \\
\hline 5. Experience and training & $\begin{array}{l}\text { What experience or training did the researcher } \\
\text { have? }\end{array}$ & \#24 (4.3.3) \\
\hline \multicolumn{3}{|l|}{ Relationship with participants } \\
\hline 6. Relationship established & $\begin{array}{l}\text { Was a relationship established prior to study } \\
\text { commencement? }\end{array}$ & $\begin{array}{l}\# 30 / 31(5.1) \\
\# 64(5.4 .1)\end{array}$ \\
\hline $\begin{array}{l}\text { 7. Participant knowledge of the } \\
\text { interviewer }\end{array}$ & $\begin{array}{l}\text { What did the participants know about the re- } \\
\text { searcher? e.g. personal goals, reasons for doing } \\
\text { the research }\end{array}$ & \#24 (4.3.2) \\
\hline 8. Interviewer characteristics & $\begin{array}{l}\text { What characteristics were reported about the } \\
\text { interviewer/facilitator? e.g. Bias, assumptions, } \\
\text { reasons and interests in the research topic }\end{array}$ & \#72 (6.2) \\
\hline \multicolumn{3}{|l|}{ Domain 2: study design } \\
\hline \multicolumn{3}{|l|}{ Theoretical framework } \\
\hline $\begin{array}{l}\text { 9. Methodological orientation } \\
\text { and Theory }\end{array}$ & $\begin{array}{l}\text { What methodological orientation was stated to } \\
\text { underpin the study? e.g. grounded theory, dis- }\end{array}$ & \#25 (4.4.1) \\
\hline
\end{tabular}




\begin{tabular}{|c|c|c|}
\hline & $\begin{array}{l}\text { course analysis, ethnography, phenomenology, } \\
\text { content analysis }\end{array}$ & \\
\hline \multicolumn{3}{|l|}{ Participant selection } \\
\hline 10. Sampling & $\begin{array}{l}\text { How were participants selected? e.g. purposive, } \\
\text { convenience, consecutive, snowball }\end{array}$ & \#22 (4.1.1) \\
\hline 11. Method of approach & $\begin{array}{l}\text { How were participants approached? e.g. face-to- } \\
\text { face, telephone, mail, email }\end{array}$ & \#23/24 (4.3.2) \\
\hline 12. Sample size & How many participants were in the study? & $\begin{array}{l}\# 30(5.1) \\
\# 59(5.4 .1)\end{array}$ \\
\hline 13. Non-participation & $\begin{array}{l}\text { How many people refused to participate or } \\
\text { dropped out? Reasons? }\end{array}$ & \#30 (5.1) \\
\hline \multicolumn{3}{|l|}{ Setting } \\
\hline 14. Setting of data collection & $\begin{array}{l}\text { Where was the data collected? e.g. home, clinic, } \\
\text { workplace }\end{array}$ & \#24 (4.3.3) \\
\hline $\begin{array}{l}\text { 15. Presence of non- } \\
\text { participants }\end{array}$ & $\begin{array}{l}\text { Was anyone else present besides the participants } \\
\text { and researchers? }\end{array}$ & $\# 24(4.3 .3)$ \\
\hline 16. Description of sample & $\begin{array}{l}\text { What are the important characteristics of the } \\
\text { sample? e.g. demographic data, date }\end{array}$ & $\# 30 / 31$ (5.1-.5.1.2) \\
\hline \multicolumn{3}{|l|}{ Data collection } \\
\hline 17. Interview guide & $\begin{array}{l}\text { Were questions, prompts, guides provided by the } \\
\text { authors? Was it pilot tested? }\end{array}$ & \#22/23 (4.1/4.3.1) \\
\hline 18. Repeat interviews & $\begin{array}{l}\text { Were repeat interviews carried out? If yes, how } \\
\text { many? }\end{array}$ & \#24 (4.3.3) \\
\hline 19. Audio/visual recording & $\begin{array}{l}\text { Did the research use audio or visual recording to } \\
\text { collect the data? }\end{array}$ & $\# 24 / 25(4.3 .3 / 4.3 .4)$ \\
\hline 20. Field notes & $\begin{array}{l}\text { Were field notes made during and/or after the } \\
\text { interview or focus group? }\end{array}$ & $\# 24(4.3 .3)$ \\
\hline 21. Duration & $\begin{array}{l}\text { What was the duration of the interviews or focus } \\
\text { group? }\end{array}$ & \#30 (5.1) \\
\hline 22. Data saturation & Was data saturation discussed? & $\begin{array}{l}\# 22(4.1 .1) \\
\# 26(4.4 .2)\end{array}$ \\
\hline 23. Transcripts returned & $\begin{array}{l}\text { Were transcripts returned to participants for } \\
\text { comment and/or correction? }\end{array}$ & \#25 (4.4.1) \\
\hline $\begin{array}{l}\text { Domain 3: analysis and find- } \\
\text { ings }\end{array}$ & & \\
\hline
\end{tabular}




\begin{tabular}{|l|l|l|}
\hline Data analysis & & \\
\hline 24. Number of data coders & How many data coders coded the data? & $\# 28$ (4.4.2.5) \\
\hline $\begin{array}{l}\text { 25. Description of the coding } \\
\text { tree }\end{array}$ & $\begin{array}{l}\text { Did authors provide a description of the coding } \\
\text { tree? }\end{array}$ & $\# 32$ (5.2.1 ff.) \\
\hline 26. Derivation of themes & $\begin{array}{l}\text { Were themes identified in advance or derived } \\
\text { from the data? }\end{array}$ & $\# 26$ (4.4.2) \\
\hline 27. Software & $\begin{array}{l}\text { What software, if applicable, was used to manage } \\
\text { the data? }\end{array}$ & $\# 26$ (4.4.1) \\
\hline 28. Participant checking & $\begin{array}{l}\text { Did participants provide feedback on the find- } \\
\text { ings? }\end{array}$ & $\# 71$ (6.1) \\
\hline Reporting & $\begin{array}{l}\text { Were participant quotations presented to illus- } \\
\text { trate the themes/findings? Was each quotation } \\
\text { identified? e.g. participant number }\end{array}$ & $\# 33$ (5.2.2 ff.) \\
\hline 29. Quotations presented & $\begin{array}{l}\text { Was there consistency between the data present- } \\
\text { ed and the findings? }\end{array}$ & $\# 73$ (6.3 ff.) \\
\hline $\begin{array}{l}\text { 30. Data and findings con } \\
\text { sistent }\end{array}$ & $\begin{array}{l}\text { Were major themes clearly presented in the find- } \\
\text { ings? }\end{array}$ & $\begin{array}{l}\text { Is there a description of diverse cases or discus- } \\
\text { sion of minor themes? }\end{array}$ \\
\hline 31. Clarity of major themes & $\# 73$ (6.3.1 ff.) \\
\hline
\end{tabular}




\section{$9 \quad$ Literaturverzeichnis}

AGS Choosing Wisely Workgroup (2013): American Geriatrics Society identifies five things that healthcare providers and patients should question. J Am Geriatr Soc 61, 622631

Albretsen CS (1991): Effekt av tilbakemelding og selvevaluering på forskrivning av beroligende midler. Holdningen hos leger ved forskrivning av benzodiazepiner: Effect of feedback and self-evaluation on prescription of addictive agents. Position of physicians about prescribing bensodiazepines. Tidsskr Nor Laegeforen $\underline{111}, 2775$

American Geriatrics Society (2012): American Geriatrics Society updated Beers Criteria for potentially inappropriate medication use in older adults. J Am Geriatr Soc $\underline{60}$, 616-631 American Geriatrics Society (2015): American Geriatrics Society 2015 Updated Beers Criteria for Potentially Inappropriate Medication Use in Older Adults. J Am Geriatr Soc $\underline{63}$, $2227-2246$

American Psychiatric Association: Diagnostic and statistical manual of mental disorders: DSM-5. 5. edition; American Psychiatric Publ, Washington, DC 2013

Anthierens S, Pasteels I, Habraken H, Steinberg P, Declercq T, Christiaens T (2010): Barriers to nonpharmacologic treatments for stress, anxiety, and insomnia: family physicians' attitudes toward benzodiazepine prescribing. Can Fam Physician 뜨, 406

Apothekenkammer Hamburg: Verordnung von Benzodiazepinen und deren Analoga: Gemeinsame Handlungsempfehlung von Ärztekammer Hamburg, Kassenärztlicher Vereinigung Hamburg und Apothekerkammer. Apothekenkammer Hamburg, Hamburg 2011 Ärzte Zeitung: Patienten-Information Das grüne Rezept. [https://www.aerztezeitung.de /extras/patienteninfo/info-gruenes-rezept/Patienteninfo_GruenesRezept1.pdf] PDFDokument zuletzt heruntergeladen am 12.02.2018

AWMF: [www.awmf.org/awmf-online-das-portal-der-wissenschaftlichen-medizin/awmfaktuell.html] Abgerufen am: 12.02.2018

Bachmann CS, Berg EA, Spigset O, Slørdal L (2008): Benzodiazepinliknende hypnotika-holdninger og forskrivningspraksis blant allmennleger: Benzodiazepine-like hypnotics-attitudes and prescription practice among general practitioners. Tidsskr Nor Laegeforen $\underline{128}, 166-170$

Barbui C, Campomori A, Mezzalira L, Lopatriello S, Cas RD, Garattini S (2001): Psychotropic drug use in Italy, 1984-99: the impact of a change in reimbursement status. Int Clin Psychopharmacol 16, 227-233 
Barrenberg E, Garbe E (2015): Use of over-the-counter (OTC) drugs and perceptions of OTC drug safety among German adults. Eur J Clin Pharmacol 71, 1389-1396

Bendtsen P, Hensing G, McKenzie L, Stridsman AK (1999): Prescribing benzodiazepines-a critical incident study of a physician dilemma. Soc Sci Med 49, 459-467

Bilitza, KW: Psychodynamik der Sucht - Einführung. In: Bilitza KW (Hrsg.): Psychodynamik der Sucht: Psychoanalytische Beiträge zur Theorie. 2. Auflage; Vandenhoeck \& Ruprecht, Göttingen 2009, 11-24

Binder W, Kornhuber HH, Waiblinger G (1984): Benzodiazepin-Sucht, unsere iatrogene Seuche--157 Fälle von Benzodiazepin-Abhängigkeit. Offentl Gesundheitswes 46, 80-86 Bjørner T, Kjølsrød L (2002): How GPs understand patients' stories. Eur J Gen Pract $\underline{8}$, $25-30$

Bjørner T, Laerum E (2003): Factors associated with high prescribing of benzodiazepines and minor opiates. A survey among general practitioners in Norway. Scand J Prim Health Care 21, 115-120

Bleuler E: Die Ambivalenz: Festgabe zur Einweihung der Neubauten der Universität Zürich. Schulthess \& Co, Zürich 1914, 95-106

Boixet M, Batlle E, Bolibar I (1996): Benzodiazepines in primary health care: a survey of general practitioners prescribing patterns. Addiction $\underline{91}$, 549-556

Bourgeois J, Elseviers MM, Azermai M, van Bortel L, Petrovic M, Vander Stichele RR (2014): Barriers to discontinuation of chronic benzodiazepine use in nursing home residents: Perceptions of general practitioners and nurses. Eur Geriatr Med $\underline{5}, 181-187$

Bradley CP (1992a): Factors which influence the decision whether or not to prescribe: the dilemma facing general practitioners. Br J Gen Pract $\underline{42}$, 454-458

Bradley CP (1992b): Uncomfortable prescribing decisions: a critical incident study. BMJ 304, 294-296

Brody DS, Khaliq AA, Thompson TL (1997): Patients' perspectives on the management of emotional distress in primary care settings. J Gen Intern Med 12, 403-406

Bühring P (2007): Hilfe für die Verordner: Medikamentenabhängigkeit. Dtsch Arztebl 104, 985

Bundesapothekerkammer (BAK): Medikamente: Abhängigkeit und Missbrauch: Leitfaden für die apothekerliche Praxis. Bundesapothekerkammer (BAK), Berlin 2011

Bundesinstitut für Arzneimittel und Medizinprodukte: [www.bfarm.de/DE/Bundesopiumstelle/Betaeubungsmittel/_node.html] Abgerufen am: 12.02.2018 
Bundesministerium der Justiz und für Verbraucherschutz: Verordnung über den Betrieb von Apotheken (Apothekenbetriebsordnung ApBetrO). Zuletzt geändert durch Art. 11 Abs. 7 G v. 18.7.2017 I 27452017 [https://www.gesetze-im-internet.de/apobetro_1987 /ApBetrO.pdf] PDF-Dokument zuletzt heruntergeladen am 23.07.2018

Chambers CD, White OZ, Lindquist JH (1983): Physician attitudes and prescribing practices: a focus on minor tranquilizers. J Psychoactive Drugs 15, 55-59

Charles C, Whelan T, Gafni A (1999): What do we mean by partnership in making decisions about treatment? BMJ $\underline{319}, 780-782$

Chen Y-C, Kreling DH (2014): The effect of the Medicare Part D benzodiazepine exclusion on the utilization patterns of benzodiazepines and substitute medications. Res Social Adm Pharm 10, 438-447

Cheung JMY, Atternäs K, Melchior M, Marshall NS, Fois RA, Saini B (2014): Primary health care practitioner perspectives on the management of insomnia: A pilot study. Aust J Prim Health 20, 103

Cimolai N (2007): Zopiclone: is it a pharmacologic agent for abuse? Can Fam Physician $\underline{53}$, 2124-2129

Cook JM, Marshall R, Masci C, Coyne JC (2007): Physicians' perspectives on prescribing benzodiazepines for older adults: a qualitative study. J Gen Intern Med 22, 303-307

Corbin JM, Strauss AL: Weiterleben lernen: Chronisch Kranke in der Familie (Familienwelten 3). Piper, München 1993

Davidson W, Molloy W, Somers G, Bédard M (1994): Relation between physician characteristics and prescribing for elderly people in New Brunswick. CMAJ 150, 917-921

Deans HG, Skinner P (1992): Doctors' views on anxiety management in general practice. J R Soc Med $\underline{85}, 83-86$

Deutsche Hauptstelle für Suchtfragen e.V.: Benzodiazepine (Beruhigungs- und Schlafmittel): Die Sucht und ihre Stoffe: Informationsreihe über gebräuchliche Suchtstoffe, 11.70.03.17. Auflage; Hamm 2018a. [http://www.dhs.de/informationsmaterial /broschueren-und-faltblaetter.html] PDF-Dokument zuletzt heruntergeladen am 23.07.2018

Deutsche Hauptstelle für Suchtfragen e.V.: Frauen - Medikamente - Selbsthilfe. Hamm 2018b [www.dhs.de/projekte/abgeschlossene-projekte/medikamente.html] Abgerufen am: 12.02.18]

Diemert S, Ganso M, Zagermann-Muncke P, Goebel R, Schulz M (2013): AMK-Umfrage zum Missbrauch: Benzodiazepine und Z-Hypnotika. Pharm Ztg 158, 1806-1810 
Dièye AM, Sylla M, Ndiaye A, Ndiaye M, Sy GY, Faye B (2006): Benzodiazepines prescription in Dakar: a study about prescribing habits and knowledge in general practitioners, neurologists and psychiatrists. Fundam Clin Pharmacol 20, 235-238

Dilling H, Freyberger HJ: Taschenführer zur ICD-10-Klassifikation psychischer Störungen: Mit Glossar und Diagnostischen Kriterien ICD-10: DCR-10 und Referenztabellen ICD-10 v.s. DSM-IV-TR. 5. überarb. Auflage unter Berücksichtig. der German Modification (GM) der ICD-10. Huber, Bern 2010

DocMorris: [www.docmorris.de] Abgerufen am: 12.02.2018

Donoghue J, Lader M (2010): Usage of benzodiazepines: A review. Int J Psychiatry Clin Pract $14,78-87$

Duden: [www.duden.de/rechtschreibung/Ambivalenz] Abgerufen am: 12.02.2018

Dündar Y, Dodd S, Strobl J, Boland A, Dickson R, Walley T (2004): Comparative efficacy of newer hypnotic drugs for the short-term management of insomnia: a systematic review and meta-analysis. Hum Psychopharmacol 19, 305-322

Dybwad TB, Kjolsrod L, Eskerud J, Laerum E (1997): Why are some doctors highprescribers of benzodiazepines and minor opiates? A qualitative study of GPs in Norway. Fam Pract 14, 361-368

Erbguth F (2015): Epileptische Anfälle und Status epilepticus. Notfall Rettungsmed 18, $357-363$

Everitt H, McDermott L, Leydon G, Yules H, Baldwin D, Little P (2014): GPs' management strategies for patients with insomnia: A survey and qualitative interview study. Br J Gen Pract $\underline{64}$, e112-e119

Filardi AFR, AraÚjo VED, Nascimento YDA, Oliveira DR de (2017): Use of psychotropics in everyday life from the perspective of health professionals and patients: A systematic review. J Crit Rev $\underline{4}, 1$

Flick U: Qualitative Sozialforschung: Eine Einführung. 7. Auflage; rowohlts enzyklopädie im Rowohlt Taschenbuch Verlag, Reinbek bei Hamburg 2016

Flick U, Garms-Homolova V, Rohnsch G (2012): "And mostly they have a need for sleeping pills": physicians' views on treatment of sleep disorders with drugs in nursing homes. J Aging Stud 26, 484-494

Fricke U, Günther J, Niepraschk-von Dollen K, Zawinell A: Anatomisch-therapeutischchemische Klassifikation mit Tagesdosen für den deutschen Arzneimittelmarkt: Methodik der ATC-Klassifikation und DDD-Festlegung. 16. Auflage; Wissenschaftliches Institut der AOK (WIdO) im AOK-Bundesverband GbR, Berlin 2017

Gelbe Seiten: [www.gelbeseiten.de] Abgerufen am: 12.02.2018 
Gemeinsamer Bundesausschuss: Anlage III (Stand: 26. Februar 2016): Übersicht über Verordnungseinschränkungen und - ausschlüsse in der Arzneimittelversorgung durch die Arzneimittel - Richtlinie und aufgrund anderer Vorschriften ( $\$ 34$ Absatz 1 Satz 6 und Absatz 3 SGB V) 2016 [https://www.g-ba.de/downloads/83-691-466/AM-RL-III-

Verordnungseinschraenkung_2017-11-04.pdf]

Gigliotti A, Figueiredo VC, Madruga CS, Marques, Ana C P R, Pinsky I, Caetano R, da Costa e Silva, Vera Luiza, Raw M, Laranjeira R (2014): How smokers may react to cigarette taxes and price increases in Brazil: data from a national survey. BMC Public Health $\underline{14}, 327$ Glaeske G (2014): Sucht auf Privatrezept: Die Verordnung von Schlafmittel trägt zur Abhängigkeitsentwicklung bei. Dr.med.Mabuse 211, 49

Glaeske G: Medikamente 2015 - Psychotrope und andere Arzneimittel mit Missbrauchsund Abhängigkeitspotential. In: Deutsche Hauptstelle für Suchtfragen e.V. (Hrsg.): DHS Jahrbuch Sucht 2017. Pabst Science Publishers, Lengerich 2017

Glaeske G, Schulze J: Der Einsatz von Benzodiazepinen bei Menschen mit einer Demenzerkrankung. In: BARMER GEK (Hrsg.): Arzneimittelreport 2013 Auswertungsergebnisse der BARMER GEK Arzneimitteldaten aus den Jahren 2011 bis 2012. Asgard Verlagsservice $\mathrm{GmbH}$, Berlin 2013, 132-141

Glaeske G, Holzbach R: Medikamentenabhängigkeit: Suchtmedizinische Reihe Band 5. Deutsche Hauptstelle für Suchtfragen, Hamm 2015

Glaser BG, Strauss AL: The discovery of grounded theory: Strategies for qualitative research. Aldine, New Brunswick 1967

Gourlay DL, Heit HA (2009): Universal precautions revisited: managing the inherited pain patient. Pain Med 10 Suppl 2, 23

Haasen C, Holzbach R (2009): Verordnung von Benzodiazepinen. Hamb Arztebl 뜨, 12 14

Hamilton IJ, Reay LM, Sullivan FM (1990): A survey of general practitioners' attitudes to benzodiazepine overprescribing. Health Bull (Edinb) 4ㅇ, 299-303

Haw C, Stubbs J (2007): Benzodiazepines--a necessary evil? A survey of prescribing at a specialist UK psychiatric hospital. J Psychopharmacol (Oxford) 21, 645-649

Heiman EM, Wood G (1981): Patient characteristics and clinician attitudes influencing the prescribing of benzodiazepines. J Clin Psychiatry $\underline{42}, 71-73$

Hill KD, Wee R (2012): Psychotropic drug-induced falls in older people: a review of interventions aimed at reducing the problem. Drugs Aging 29, 15-30

Hoebert JM, Souverein PC, Mantel-Teeuwisse AK, Leufkens HGM, van Dijk L (2012): Reimbursement restriction and moderate decrease in benzodiazepine use in general practice. Ann Fam Med 10, 42-49 
Hoffmann F (2013a): Benefits and risks of benzodiazepines and Z-drugs: comparison of perceptions of GPs and community pharmacists in Germany. Ger Med Sci 11, 1-7

Hoffmann F (2013b): Perceptions of German GPs on benefits and risks of benzodiazepines and Z-drugs. Swiss Med Wkly $\underline{143}$, w13745

Hoffmann F, Glaeske G (2014): Benzodiazepinhypnotika, Zolpidem und Zopiclon auf Privatrezept: Verbrauch zwischen 1993 und 2012. Nervenarzt 도, 1402-1409

Hoffmann F, Glaeske G, Scharffetter W (2006): Zunehmender Hypnotikaverbrauch auf Privatrezepten in Deutschland. SUCHT $\underline{52}, 360-366$

Hoffmann F, Pfannkuche M, Glaeske G (2008): Hochverbrauch von Zolpidem und Zopiclon. Querschnittsstudie auf Basis von Krankenkassendaten. Nervenarzt 79, 67-72

Hoffmann F, Scharffetter W, Glaeske G (2009): Verbrauch von Zolpidem und Zopiclon auf Privatrezepten zwischen 1993 und 2007. Nervenarzt 0, 578-583

Hoffmann F, Hies M, Glaeske G (2010): Regional variations of private prescriptions for the non-benzodiazepine hypnotics zolpidem and zopiclone in Germany. Pharmacoepidem. Drug Safe 19, 1071-1077

Hoffmann F, Schmiemann G, Windt R (2014): Privat statt Kasse?: Einstellungen von Hausärzten und Apothekern zur Verordnung von Hypnotika. Dtsch Med Wochenschr $\underline{139}, 1153-1158$

Holden JD, Hughes IM, Tree A (1996): Attitudes of general practitioners to benzodiazepine prescribing and withdrawal. J Ment Health $\underline{5}, 501-506$

Hollister L, Motzenbecker F, Degan RO. (1961): Withdrawal reactions from chlordiazepoxide ("Librium"). Psychopharmacologia 2 , 63-68

Holzbach R: Lippstädter Benzo-Check 2018 [www.lwl-kurzlink.de/benzo-check] PDFDokument zuletzt heruntergeladen am 23.07.2018

Holzbach R, Martens M, Kalke J, Raschke P (2010): Zusammenhang zwischen Verschreibungsverhalten der Arzte und Medikamentenabhängigkeit ihrer Patienten. Bundesgesundheitsblatt Gesundheitsforschung Gesundheitsschutz 프, 319-325

Hummers-Pradier E, Scheidt-Nave C, Martin H, Heinemann S, Kochen MM, Himmel W (2008): Simply no time?: Barriers to GPs' participation in primary health care research. Fam Pract 25, 105-112

Iliffe S, Curran HV, Collins R, Yuen Kee SC, Fletcher S, Woods B (2004): Attitudes to long-term use of benzodiazepine hypnotics by older people in general practice: findings from interviews with service users and providers. Aging Ment Health $\underline{8}$, 242-248 Isacson D, Bingefors K, Wennberg M, Dahlstrom M (1993): Factors associated with highquantity prescriptions of benzodiazepines in Sweden. Soc Sci Med $\underline{36}$, 343-351 
Jamison RN, Sheehan KA, Scanlan E, Matthews M, Ross EL (2014): Beliefs and attitudes about opioid prescribing and chronic pain management: survey of primary care providers. J Opioid Manag $\underline{10}, 375-382$

Johnson C, Baxter B, Brough R, Buchanan J (2007): Benzodiazepine prescribing: Lessons from interprofessional dialogue. Aust Fam Physician $\underline{36}$, 245-246

Jonas K, Broemer P, Diehl M (2000): Attitudinal ambivalence. Eur Rev Soc Psychol 11, $35-$ 74

Kapil V, Green JL, Le Lait C, Wood DM, Dargan PI (2014): Misuse of benzodiazepines and Z-drugs in the UK. Br J Psychiatry $\underline{205}$, 407-408

Kassenärztliche Bundesvereinigung: Bundesmantelvertrag-Ärzte 2017 [https://www.gkvspitzenverband.de/krankenversicherung/aerztliche_versorgung/bundesmantelvertrag/ bundesmantelvertrag.jsp] PDF-Dokument zuletzt heruntergeladen am 23.07.2018

Kassenärztliche Vereinigung Nordrhein: Benzodiazepine, Zopiclon und Zolpidem - anwenden und absetzen. VIN Verordnungsinfo Nordrhein. Kassenärztliche Vereinigung Nordrhein, Düsseldorf 2013

Kiehn J (2009): Nicht ohne Indikation: Problematische Verordnung von Benzodiazepinen aus berufsrechtlicher Sicht. Hamb Arztebl 06, 16

Knopf H, Melchert H-U: Bundes-Gesundheitssurvey: Arzneimittelgebrauch - Konsumverhalten in Deutschland (Beiträge zur Gesundheitsberichterstattung des Bundes; hrsg. v. Robert Koch-Institut). Saladruck, Berlin 2003

Kristensen P, Fjermestad T (1991): Benzodiazepiner--holdninger og forskrivningspraksis blant allmennpraktikere og psykiatere: Benzodiazepines--attitudes and prescription practice among general practitioners and psychiatrists. Tidsskr Nor Laegeforen 111, 2241-2245

Lader M (1998): Iatrogenic sedative dependence and abuse--have doctors learnt caution? Addiction 93, 1133-1135

Lader M (2011): Benzodiazepines revisited--will we ever learn? Addiction 106, 2086-2109 Lohse MJ, Müller-Oerlinghausen B: Hypnotika und Sedativa. In: Schwabe U, Paffrath D (Hrsg.): Arzneiverordnungs-Report 2016: Aktuelle Daten, Kosten, Trends und Kommentare. Springer Verlag, Berlin Heidelberg 2016, 499-508

Lüscher, K: Ambivalenz: Eine soziologische Annäherung. In: Walter D, Lüscher K, Müller C (Hrsg.): Ambivalenzen erkennen, aushalten und gestalten. Theologischer Verlag (TVZ), Zürich 2009, 17-62

Markota M, Rummans TA, Bostwick JM, Lapid MI (2016): Benzodiazepine Use in Older Adults: Dangers, Management, and Alternative Therapies. Mayo Clin Proc 91, 1632-1639 
Markus L (2007): Flunitrazepam: Erfahrungsbericht mit einem gefährlichen Arzneimittel. Ärzteblatt Sachsen $\underline{5}, 224-226$

Maust DT, Kales HC, Wiechers IR, Blow FC, Olfson M (2016): No End in Sight: Benzodiazepine Use in Older Adults in the United States. J Am Geriatr Soc 64, 2546-2553

MAXQDA: [www.maxqda.de] Abgerufen am: 12.02.2018

Medizin ohne Rezept: [www.medizin-ohne-rezept.com] Abgerufen am: 12.02.2018

Mey G, Mruck K: Methodologie und Methodik der Grounded Theory. In: Kempf W, Kiefer M (Hrsg.): Forschungsmethoden der Psychologie: Zwischen naturwissenschaftlichem Experiment und sozialwissenschaftlicher Hermeneutik. (Hochschullehrbücher Band 3), 1. Auflage; Regener, Berlin 2009, 100-150

Monette J, Tamblyn RM, McLeod PJ, Gayton DC (1997): Characteristics of physicians who frequently prescribe long-acting benzodiazepines for the elderly. Eval Health Prof $\underline{20}$, $115-130$

Morabia A, Fabre J, Dunand JP (1992): The influence of patient and physician gender on prescription of psychotropic drugs. J Clin Epidemiol $\underline{45}, 111-116$

Morrice A, Iliffe S (1992): Reducing benzodiazepine use in patients requesting repeat prescriptions in general practice. Med Sci Res 20, 535-536

Müller-Oerlinghausen B (1986): Prescription and misuse of benzodiazepines in the Federal Republic of Germany. Pharmacopsychiat 19, 8-13

Nissen C, Frase L, Hajak G, Wetter TC (2014): Hypnotika--Stand der Forschung. Nervenarzt $\underline{85}, 67-76$

Nowell PD, Mazumdar S, Buysse DJ, Dew MA, Reynolds CF, Kupfer DJ (1997): Benzodiazepines and zolpidem for chronic insomnia: a meta-analysis of treatment efficacy. JAMA $\underline{278}, 2170-2177$

Onlinewarnungen: [www.onlinewarnungen.de] Abgerufen am: 12.02.2018

Owston CL (2016): The inherited patient on opioids: A challenge for primary care clinicians. JAAPA $\underline{29}, 37-43$

Paulozzi LJ, Jones CM, Mack KA, Rudd RA: Vital Signs: Overdoses of Prescription Opioid Pain Relievers: United States, 1999-2008, Center for Disease Control and Prevention Morbidity and Mortality Weekly Report. Office of Surveillance, Epidemiology, and Laboratory Services, Centers for Disease Control and Prevention (CDC), U.S. Department of Health and Human Services, Atlanta 2011

Peters-Klimm F, Hermann K, Gágyor I, Haasenritter J, Bleidorn J (2013): Erfahrungen und Einstellungen zu Klinischen Studien in der Hausarztpraxis: Ergebnisse einer Befragung von deutschen Hausärzten. Gesundheitswesen $\underline{75}$, 321-327 
Pfizer: Fachinformation Pfizer Tavor ${ }^{\circledR}$ 0,5 mg/1,0 mg/Tabs 2,0 mg/2,5 mg'Tabletten (Stand Juni 2016) [https://www.pfizermed.de/fileadmin/produktdatenbank/pdf/ 002029_freigabe.pdf]

Puustinen J, Nurminen J, Vahlberg T, Lyles A, Isoaho R, Räihä I, Kivelä S-L (2012): CNS medications as predictors of precipitous cognitive decline in the cognitively disabled aged: a longitudinal population-based study. Dement Geriatr Cogn Dis Extra 2, 57-68

QUARC: [www.quarc.de] Abgerufen am: 12.02.2018

ratiopharm GmbH: Fachinformation Flunitrazepam-ratiopharm ${ }^{\circledR} 1 \mathrm{mg}$ (Stand Februar 2014)[https://s3.eu-central-1.amazonaws.com/prod-cerebro-ifap/media_all/51977.pdf]

Riklin F (1911): Bericht über Vorträge und Diskussionen psychoanalytischens Charakters: Vortrag von Prof. Bleuler-Zürich über Ambivalenz. Zentralbl Psychoanalyse 1, 266-268

Rogers A, Pilgrim D, Brennan S, Sulaiman I, Watson G, Chew-Graham C (2007): Prescribing benzodiazepines in general practice: a new view of an old problem. Health (London) 11, 181-198

Rosemann T, Szecsenyi J (2004): General practitioners' attitudes towards research in primary care: qualitative results of a cross sectional study. BMC Fam Pract $\underline{5}, 31$

Rote Liste: [www.online.rote-liste.de] Abgerufen am: 12.02.2018

Rummans TA, Davis LJ, Morse RM, Ivnik RJ (1993): Learning and memory impairment in older, detoxified, benzodiazepine-dependent patients. Mayo Clin Proc 68, 731-737

S1-Leitlinie Insomnie: Deutsche Gesellschaft für Neurologie 2012. AWMF-Reg. Nr. 030045 [https://www.awmf.org/uploads/tx_szleitlinien/030-0451_S1_Insomnie_2012abgelaufen.pdf]

S3-Leitlinie Behandlung von Angststörungen (2014). AWMF-Reg. Nr. 051-028 [https://www.awmf.org/uploads/tx_szleitlinien/051-028k_S3_Angststörungen_201405_1_01.pdf]

S3-Leitlinie Nicht erholsamer Schlaf/Schlafstörungen (2009). Somnologie 13, 1-160

S3-Leitlinie Nicht erholsamer Schlaf/Schlafstörungen (2017): Kapitel "Insomnie bei Erwachsenen" Update 2016. AWMF-Reg. Nr. 063-003. Somnologie 21, 2-44

S3-Leitlinie Sedierung in der gastrointestinalen Endoskopie (2014). AWMF-Reg. Nr. 021 014 [https://www.awmf.org/uploads/tx_szleitlinien/021-0141_S3_Sedierung _gastrointestinale_Endoskopie_2015-07.pdf]

S3-Leitlinie Unipolare Depression: Kurzfassung, 2. Auflage. Version 1 (2017). AWMFReg. Nr. nvl-005 [https://www.awmf.org/uploads/tx_szleitlinien/nvl005k_Unipolare_Depression-2018-02.pdf] 
Sällström G, Maasing R (1980): Swedish company doctors' attitudes to benzodiazepines and neuroleptics used as minor tranquillizers. Pharmatherapeutica 2 , 380-386

Schaufler J, Telschow C: Überblick über die Arzneiverordnungen nach Arztgruppen. In: Schwabe U, Paffrath D (Hrsg.): Arzneiverordnungs-Report 2015: Aktuelle Daten, Kosten, Trends und Kommentare. Springer Verlag, Berlin Heidelberg 2015, 1079-1090

Schaufler J, Telschow C: Überblick über die Arzneiverordnungen nach Arztgruppen. In: Schwabe U, Paffrath D (Hrsg.): Arzneiverordnungs-Report 2016: Aktuelle Daten, Kosten, Trends und Kommentare. Springer Verlag, Berlin Heidelberg 2016, 753-762

Schmidt M: Benzodiazepine. In: Vagts DA (Hrsg.): Suchtmittel in der AINS. Springer Medizin Verlag, Heidelberg 2007, 79-89

Schmiedl S, Rottenkolber M, Hasford J, Rottenkolber D, Farker K, Drewelow B, Hippius M, Saljé K, Thürmann P (2014): Self-medication with over-the-counter and prescribed drugs causing adverse-drug-reaction-related hospital admissions: results of a prospective, long-term multi-centre study. Drug Saf $\underline{37}, 225-235$

Schulz M (2014): Verordnung von Hypnotika und Tranquillanzien für GKV-Versicherte auf Privatrezept: Rahmenbedingungen kontra Versorgungsrealität? Dtsch Med Wochenschr $\underline{139}, 1151-1152$

Seamark D, Seamark C, Greaves C, Blake S (2013): GPs prescribing of strong opioid drugs for patients with chronic non-cancer pain: a qualitative study. Br J Gen Pract $\underline{63}$, e821-8 Singler A, Treutlein G: Historische und soziologische Aspekte abweichenden Verhaltens im Spitzensport: Das Beispiel des Dopings in der Bundesrepublik Deutschland. In: Hofmann AR, Krüger M (Hrsg): Olympia als Bildungsidee: Beiträge zur olympischen Geschichte und Pädagogik. (Bildung und Sport 2). Springer VS, Wiesbaden 2013, 157-182

Sirdifield C, Anthierens S, Creupelandt H, Chipchase SY, Christiaens T, Siriwardena A (2013): General practitioners' experiences and perceptions of benzodiazepine prescribing: Systematic review and meta-synthesis. BMC Fam Pract 14, 191

Sirdifield C, Chipchase SY, Owen S, Siriwardena AN (2017): A Systematic Review and Meta-Synthesis of Patients' Experiences and Perceptions of Seeking and Using Benzodiazepines and Z-Drugs: Towards Safer Prescribing. Patient 10, 1-15

Siriwardena AN, Qureshi Z, Gibson S, Collier S, Latham M (2006): GPs' attitudes to benzodiazepine and 'Z-drug' prescribing: a barrier to implementation of evidence and guidance on hypnotics. Br J Gen Pract 6ㅜ, 964-967

Siriwardena AN, Qureshi MZ, Dyas JV, Middleton H, Orner R (2008): Magic bullets for insomnia?: Patients' use and experiences of newer ( $\mathrm{Z}$ drugs) versus older (benzodiazepine) hypnotics for sleep problems in primary care. Br J Gen Pract $\underline{58}$, 417-422 
Siriwardena AN, Apekey T, Tilling M, Dyas JV, Middleton H, Ørner R (2010): General practitioners' preferences for managing insomnia and opportunities for reducing hypnotic prescribing. J Eval Clin Pract $\underline{16}, 731-737$

Souza-Pinto LFS, Castilho VM, Brandão ML, Nobre MJ (2007): The blockade of AMPAkainate and NMDA receptors in the dorsal periaqueductal gray reduces the effects of diazepam withdrawal in rats. Pharmacol Biochem Behav $\underline{87}, 250-257$

Spitzenverband Bund der Krankenkassen und Deutscher Apothekerverband: Rahmenvertrag über die Arzneimittelversorgung nach \129 Absatz 2 SGB V 2016[https:/ /www.gkvspitzenverband.de/media/dokumente/krankenversicherung_1/arzneimittel /rahmenvertraege/apotheken/AM_20160930_Rahmenvertrag_129_Absatz-2_SGB-V.pdf] PDF-Dokument zuletzt heruntergeladen am 23.07.2018

Sponsel R: Ambivalenz: Erfindung und Darstellung des Begriffs durch Eugen Bleuler. Bericht vom Vortrag 1911 und Veröffentlichung 1914. Medizinische Psychosomatik, Psychopathologie und Psychiatrie, Erlangen 2002. IP-GIPT: [http://www.sgipt.org/medppp /gesch/ambiv-g.htm]

Srisurapanont M, Garner P, Critchley J, Wongpakaran N (2005): Benzodiazepine prescribing behaviour and attitudes: a survey among general practitioners practicing in northern Thailand. BMC Fam Pract $\underline{6}, 27$

Steinbach M (1968): Über den Einfluss anaboler Wirkstoffe auf Körpergewicht, Muskelkraft und Muskeltraining. Sportarzt und Sportmedizin XIX/1968, 485-492

Stilnox: [www.mein.sanofi.de/produkte/Stilnox/faq] Abgerufen am: 12.02.2018

Strauss A, Corbin J: Grounded theory: Grundlagen qualitativer Sozialforschung. Unveränd. Nachdruck der letzten Auflage von 1996; Beltz, Weinheim 2010

Strauss AL: Grundlagen qualitativer Sozialforschung: Datenanalyse und Theoriebildung in der empirischen soziologischen Forschung (UTB für Wissenschaft Uni-Taschenbücher 1776, Soziologie). Fink, München 1998

Ströhle A, Antonijevic IA, Steiger A, Sonntag A (1999): Abhängigkeit von "NonBenzodiazepinhypnotika." Zwei Fallberichte. Nervenarzt 70, 72-75

Subelj M, Vidmar G, Svab V (2010): Prescription of benzodiazepines in Slovenian family medicine: a qualitative study. Wien Klin Wochenschr 122, 474-478

Substance Abuse and Mental Health Services Administration: Results from the $2009 \mathrm{Na}$ tional Survey on Drug Use and Health: Volume I. Summary of National Findings (Office of Applied Studies, NSDUH Series H-38A, HHS Publication No. SMA 10-4586 Findings). Rockville, MD 2010

Summers J, Brown KW (1998): Benzodiazepine prescribing in a psychiatric hospital. Psychiatric Bulletin 22, 480-483 
Templeton L, Deehan A, Taylor C, Drummond C, Strang J (1997): Surveying general practitioners: does a low response rate matter? Br J Gen Pract 47, 91-94

Thomae H: Der Mensch in der Entscheidung. J. A. Barth, München 1960

Tong A, Sainsbury P, Craig J (2007): Consolidated criteria for reporting qualitative research (COREQ): A 32-item checklist for interviews and focus groups. Int J Qual Health Care $\underline{19}$, 349-357

Troest A (1993): Ordination af benzodiazepinpraeparater i Nykøbing Falster Kommune. En receptundersøgelse med henblik på ordinationsvaner i almen praksis: Prescriptions of benzodiazepines in the municipality of Nykøbing Falster. A study of prescriptions with reference to prescription habits in general practice. Ugeskr Laeg 155, 2029-2032

Verger P, Aulagnier M, Protopopescu C, Villani P, Gourrheux J-C, Bouvenot G, Paraponaris A (2004): Hypnotic and tranquillizer use among general practitioners in south-eastern France and its relation to occupational characteristics and prescribing habits. Fundam Clin Pharmacol $\underline{18}, 379-385$

Voelker K (2009): Nur bis zu acht Wochen: Welche Vorgaben gelten für die Verordnung von Benzodiazepinen? Hamb Arztebl 06, 15

Wagner AK, Ross-Degnan D, Gurwitz JH, Zhang F, Gilden DB, Cosler L, Soumerai SB (2007): Effect of New York State regulatory action on benzodiazepine prescribing and hip fracture rates. Ann Intern Med 146, 96-103

Weaver MF (2015): Prescription Sedative Misuse and Abuse. Yale J Biol Med ㅁ8, 247-256 Weisberg RB, Dyck I, Culpepper L, Keller MB (2007): Psychiatric treatment in primary care patients with anxiety disorders: a comparison of care received from primary care providers and psychiatrists. Am J Psychiatry 164, 276-282

Weiß V, Hauswaldt J (2015): Verordnungshäufigkeit von Schlafmitteln auf Privatrezept: Analyse aus hausärztlichen Routinedaten. Z Allg Med 1ㅗ, 408-412

WIdO/DIMDI: Anatomisch-therapeutisch-chemische Klassifikation mit Tagesdosen. Amtliche Fassung des ATC-Index mit DDD-Angaben für Deutschland 2016. [https://www.wido.de/amtl_atc-code.html] PDF-Dokument zuletzt heruntergeladen am 23.07.2018

Wolde GB, Dijkstra A, van Empelen P, Knuistingh Neven A, Zitman FG (2008): Psychological determinants of the intention to educate patients about benzodiazepines. Pharm World Sci $\underline{30}$, 336-342

Wollny A, Marx G (2009): Qualitative Sozialforschung - Ausgangspunkte und Ansätze für eine forschende Allgemeinmedizin: Teil 2: Qualitative Inhaltsanalyse vs. Grounded Theory. Z Allg Med 모, 467-476 
World Health Organization: The ICD-10 Classification of Mental and Behavioural Disorders: Clinical descriptions and diagnostic guidelines: F10-F19: Mental and behavioural disorders due to psychoactive substance use. World Health Organization, Genf 1992

Xing D, Ma XL, Ma JX, Wang J, Yang Y, Chen Y (2014): Association between use of benzodiazepines and risk of fractures: a meta-analysis. Osteoporos Int $\underline{25}, 105-120$

Xu X, Pesko MF, Tynan MA, Gerzoff RB, Malarcher AM, Pechacek TF (2013): Cigarette price-minimization strategies by U.S. smokers. Am J Prev Med 44, 472-476

Zandstra SM, van Rijswijk E, Rijnders CAT, Van De Lisdonk, E H, Bor JHJ, van Weel C, Zitman FG (2004): Long-term benzodiazepine users in family practice: differences from short-term users in mental health, coping behaviour and psychological characteristics. Fam Pract 21, 266-269

Zisselman MH, Rovner BW, Yuen EJ, Sholevar D (1997): Physician rationale for benzodiazepine prescriptions to elderly hospitalized patients. Am J Geriatr Psychiatry $\underline{5}, 167-171$ 


\section{Danksagung}

Mein Dank gehört meiner Doktormutter Frau Prof. Dr. med. Eva Hummers-Pradier, sowohl für die thematische Anregung zu dieser Arbeit als auch für die gute Betreuung, die sich insbesondere durch eine unkomplizierte Erreichbarkeit auszeichnete und mir den zeitigen Abschluss dieses Projektes ermöglichte. Ebenso möchte ich meinem Ko-Betreuer Herrn Prof. Dr. med. Jürgen Brockmöller danken, der mit seinen manchmal auch kritischen Anregungen dazu beigetragen hat, diese Arbeit kontinuierlich zu verbessern. Ein großes Dankeschön gebührt Herrn Prof. Dr. Wolfgang Himmel, der mich mit zahlreichen praktischen, organisatorischen und inhaltlichen Tipps unterstützt hat. Kristina Meier M.A. vom Methodenzentrum Sozialwissenschaften sei gedankt für ihre Hilfe bei der Auswertung der Daten. Und natürlich möchte ich den Personen danken, ohne die diese Arbeit nicht möglich gewesen wäre: allen Ärztinnen und Ärzten, die sich die Zeit für ein Interview genommen haben! 